\title{
The Statistical Theory of Quantum Dots
}

\author{
Y. Alhassid \\ Center for Theoretical Physics, Sloane Physics Laboratory, Yale University, New Haven, Connecticut 06520, USA
}

A quantum dot is a sub-micron-scale conducting device containing up to several thousand electrons. Transport through a quantum dot at low temperatures is a quantum-coherent process. This review focuses on dots in which the electron's dynamics are chaotic or diffusive, giving rise to statistical properties that reflect the interplay between one-body chaos, quantum interference, and electronelectron interactions. The conductance through such dots displays mesoscopic fluctuations as a function of gate voltage, magnetic field, and shape deformation. The techniques used to describe these fluctuations include semiclassical methods, random-matrix theory, and the supersymmetric nonlinear $\sigma$ model. In open dots, the approximation of noninteracting quasiparticles is justified, and electron-electron interactions contribute indirectly through their effect on the dephasing time at finite temperature. In almost-closed dots, where conductance occurs by tunneling, the charge on the dot is quantized, and electron-electron interactions play an important role. Transport is dominated by Coulomb blockade, leading to peaks in the conductance that at low temperatures provide information on the dot's ground-state properties. Several statistical signatures of electronelectron interactions have been identified, most notably in the dot's addition spectrum. The dot's spin, determined partly by exchange interactions, can also influence the fluctuation properties of the conductance. Other mesoscopic phenomena in quantum dots that are affected by the charging energy include the fluctuations of the cotunneling conductance and mesoscopic Coulomb blockade.

[Published in Rev. Mod. Phys. 72, 895 (2000)]

\section{CONTENTS}

I. Introduction

A. Fabrication and physical parameters of quantum dots

B. From "regular" to chaotic dots

C. From disordered to chaotic ballistic dots

D. From open to closed dots

E. From noninteracting to interacting electrons

F. Methods

G. Outline of the review

II. Electron Transport Through Quantum Dots

A. Quasiclassical description of conductivity

B. Conductance in mesoscopic systems and the Landauer formula

C. $R$-matrix formalism

D. Resonant tunneling

E. Coulomb blockade

F. Cotunneling

G. Nonlinear transport

III. Statistical Theory: from Disordered Metals to Ballistic Dots

A. Disordered metals and ballistic dots

1. Scales in the diffusive regime

2. Scales in the ballistic regime

3. Models of disordered structures and ballistic dots

B. The semiclassical approach

1. Spectral correlations in chaotic and disordered systems

2. Conductance fluctuations in disordered metals

C. The universal regime: random-matrix theory

1. Gaussian ensembles

2. Crossover ensembles

3. Gaussian processes

4. Circular ensembles
D. The supersymmetry method

29

IV. Mesoscopic Fluctuations in Open Dots
2

4

6

7

9

9

9

10

10

11

12

13

14

17

17

18

18

18

19

19

20

20

22

22

23

26

27

29
A. The random-matrix approach

B. The semiclassical approach

C. Mesoscopic fluctuations of the conductance

1. Conductance distributions

2. Weak localization

3. Ericson fluctuations

4. Parametric correlations

D. Conductance fluctuations at finite temperature

E. Dephasing

1. Models for dephasing

2. Temperature dependence of dephasing

3. Conductance distributions

V. Mesoscopic Fluctuations in Closed Dots

A. Statistical theory at low temperatures

B. Conductance peak statistics

1. Partial-width amplitude distribution

2. Width distribution

3. Peak-height distributions

C. Parametric correlations of the conductance peaks

D. Crossover from conserved to broken time-reversal symmetry

1. Conductance peak distributions

2. Weak localization

E. Peak-spacing statistics

F. Finite-temperature statistics

1. Conductance peaks

2. Peak spacings

G. Spectral scrambling

H. Correlations between the addition and excitation spectra

VI. Interaction Effects

A. Peak-spacing statistics and interactions

1. Hartree-Fock approximation and Koopmans' theorem 
2. Random-phase approximation in disordered dots

3. Parametric variation of the mean field

4. Anderson model with interactions

B. Spin effects and interactions

C. Peak-height statistics and interactions

D. Random interaction matrix model

VII. Charging Energy Effects in Quantum Dots

A. Mesoscopic fluctuations in elastic cotunneling

B. Mesoscopic Coulomb blockade

C. Mesoscopic fluctuations of the differential capacitance

VIII. Conclusion and Future Directions

IX. Acknowledgments

References

\section{INTRODUCTION}

Recent advances in materials science have made possible the fabrication of small conducting devices known as quantum dots, where up to several thousand electrons are confined to a region whose linear size is about $0.1-1 \mu \mathrm{m}$ (Kastner, 1992). Quantum dots are typically made by forming a two-dimensional electron gas in the interface region of a semiconductor heterostructure and applying an electrostatic potential to metal gates to further confine the electrons to a small region ("dot") in the interface plane. Because the electronic motion is restricted in all three dimensions, a quantum dot is sometimes referred to as a zero-dimensional system. The transport properties of a quantum dot can be measured by coupling it to leads and passing current through the dot. The electron's phase is preserved over distances that are large compared with the size of the system, giving rise to new phenomena not observed in macroscopic conductors. As the name suggests, conductance through a quantum dot is characterized by quantum coherence.

Quantum dots belong to a larger class of systems, termed mesoscopic by van Kampen (1981), which are intermediate between microscopic systems, such as nuclei and atoms, and macroscopic bulk matter (Akkermans et al., 1995). A system is called mesoscopic when the electron's phase coherence length $L_{\phi}$ (the typical distance the electron travels without losing phase coherence) is larger than or comparable to the system's size $L$. Phase coherence is affected by the coupling of the electron to its environment, and phase-breaking processes involve a change in the state of the environment. In most cases phase coherence is lost in inelastic scatterings, e.g., with other electrons or phonons, but spin-flip scattering from magnetic impurities can also contribute to phase decoherence. Elastic scatterings of the electron, e.g., from impurities, usually preserve phase coherence and are characterized by the elastic mean free path $l . L_{\phi}$ increases rapidly with decreasing temperature, and for $L \sim 1 \mu \mathrm{m}$, an open system typically becomes mesoscopic below $\sim 100 \mathrm{mK}$. In a mesoscopic sample, the description of transport in terms of local conductivity breaks down, and the whole sample must be treated as a single, coherent entity.

The field of mesoscopic physics originated in the study of disordered systems in which the electron's motion is diffusive, i.e., $l$ is small relative to $L$. In the late 1980 s it became possible to produce high-mobility semiconductor microstructures that were sufficiently small and free of impurities to ensure that the mean free path $l$ exceeds the system's size $L$. Such devices are termed ballistic. Transport in a ballistic quantum dot is dominated by electronic scattering not from impurities, but from the structure's boundaries. Most experimental research on quantum dots is focused on ballistic dots.

The coupling between a quantum dot and its leads can be experimentally controlled. In an open dot, the coupling is strong and the movement of electrons across the dot-lead junctions is classically allowed. But when the point contacts are pinched off, effective barriers are formed and conductance occurs only by tunneling. In these almost-isolated or closed quantum dots, the charge on the dot is quantized, and the dot's low-lying energy levels are discrete, with widths smaller than their spacing. Closed dots have been called "artificial atoms" (Kastner, 1993; Ashoori, 1996) because of their discrete excitation spectra.

In the past, experimental studies of quantum phenomena in small systems were limited to natural systems such as atoms and nuclei. Quantum dots are man-made structures small enough to be governed by the laws of quantum mechanics. The advantage of these artificial systems is that their transport properties are readily measured, with the strength of the dot-lead couplings, the number of electrons in the dot, and the dot's size and shape all under experimental control. Furthermore, effects of time-reversal symmetry breaking are easily measured by applying a magnetic field.

Quantum dots are not the only miniature structures whose transport properties have been measured. Similar experiments have been performed recently on even smaller structures such as very clean metallic nanoparticles (Ralph, Black, and Tinkham, 1997; Davidović and Tinkham, 1999), $C_{60}$ molecules deposited on gold substrate (Porath and Millo, 1996), and carbon nanotubes (Bockrath et al., 1997; Tans et al., 1997; Cobden et al., 1998). Some of the phenomena observed in these systems are striking similar to those seen in quantum dots, suggesting that quantum dots are generic systems for exploring the physics of small, coherent quantum structures.

\section{A. Fabrication and physical parameters of quantum dots}

There are two types of quantum dots, corresponding to lateral and vertical geometries. In the more 
common lateral dot, the current flows within the plane to which the electrons are confined; in a vertical dot (Reed et al., 1988), the current flows perpendicular to the plane. Quantum dots are produced by several techniques. A typical example of a lateral dot and its fabrication method are illustrated in Fig. 1(a): on the right is an electron micrograph of the dot and on the left is a schematic drawing. A layer of AlGaAs is grown on top of a layer of GaAs by molecular-beam epitaxy. Electrons accumulate at the GaAs/AlGaAs interface to form a two-dimensional electron gas (their motion in the vertical direction is confined to the lowest state of a quantum well). Metal gates (lighter regions in the micrograph) are created at the top of the structure by electron-beam lithography. A negative bias applied to the top metal gate depletes the electrons under the gate and restricts them to a small region (the dark central region in the micrograph). The dot is coupled to the bulk $2 \mathrm{D}$ electrongas regions by two individually adjustable point contacts. A voltage $V_{s d}$ applied between the source and the drain drives a current $I$ through the device. The linear conductance is determined from $G=I / V_{s d}$ in the limit of small $V_{s d}$. The shape and size of the dot can be controlled by voltages $V_{g 1}$ and $V_{g 2}$ applied to two shape-distorting gates. The ability to control the dot-lead couplings as well as the dot's shape and area allows us to study a continuous range of physically interesting situations.

A schematic view of another lateral dot (Oosterkamp et al., 1997) is shown in Fig. 1(b). The lighter areas represent the metal gates. The darker area contains the electrons: the central region is the dot itself, connected by point contacts to the large 2D electron-gas regions on the left and right. The left and right pairs of gates control the dot's barriers (i.e., its degree of openness), while the central pair of gates is used to vary its shape and size.

The confined electrons are typically $\sim 50-100 \mathrm{~nm}$ below the surface. The effective mass of an electron in GaAs is rather low: $m^{*}=0.067 m_{e}$. A typical sheet density of $n_{s} \sim 4 \times 10^{11} \mathrm{~cm}^{-2}$ corresponds to a Fermi wavelength of $\lambda_{F}=\left(2 \pi / n_{s}\right)^{1 / 2} \sim 40 \mathrm{~nm}$ (about two orders of magnitude larger than in a metal) and Fermi energy of $E_{F} \sim$ $14 \mathrm{meV}$. The mobility of GaAs/AlGaAs heterostructures is in the range $\mu_{e} \sim 10^{4}-10^{6} \mathrm{~cm}^{2} / \mathrm{V} \cdot \mathrm{s}$, leading to a typical mean free path of $l=v_{F} m^{*} \mu_{e} / e \sim 0.1-10 \mu \mathrm{m}\left(v_{F}\right.$ is the Fermi velocity). Electron transport in submicron dots with the higher mobility values is thus ballistic. To observe quantum coherence effects it is usually necessary to have a mean single-particle level spacing $\Delta$ in the dot that is comparable to or larger than the temperature. For a dot with an effective area of $\mathcal{A} \sim 0.3 \mu \mathrm{m}^{2}$, the spacing $\Delta=\pi \hbar^{2} / m^{*} \mathcal{A} \sim 11 \mu \mathrm{eV}$ can be resolved at temperatures of $\sim 100 \mathrm{mK}$ (corresponding to $k T=8.6 \mu \mathrm{eV}$ ). The lowest effective electron temperatures attained using dilution refrigerators are $\sim 50 \mathrm{mK}$.
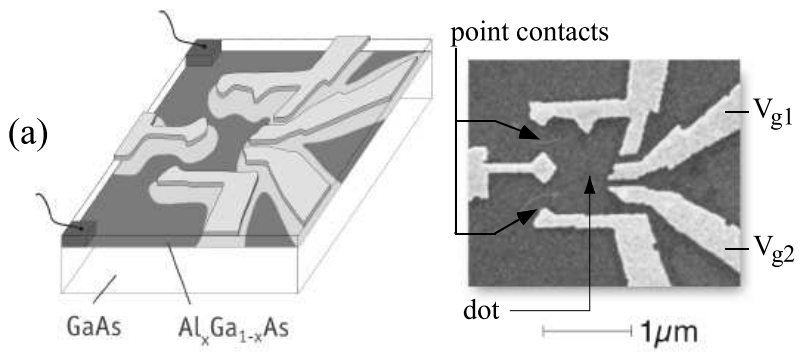

(b)

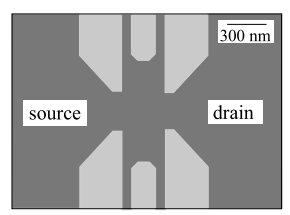

FIG. 1. Quantum dots: (a) a quantum dot used by Folk et al. (1996). On the right is a scanning electron micrograph of the dot (top view), and on the left is a schematic drawing of the device. Electrons are trapped vertically in the interface of a GaAs/AlGaAs heterostructure, and form a 2D electron gas (darker area). Their lateral confinement to the dot region is achieved by applying a negative voltage to the top metal gate (lighter shade), depleting the electrons underneath. The dot is coupled to two leads (source and drain) through point contacts. Two gate voltages $V_{g 1}$ and $V_{g 2}$ can be varied to change the shape and area of the dot. (b) A diagram of a micrograph of another dot by Oosterkamp et al. (1997). The darker area includes the dot region (center) and the two large 2D electron-gas areas on the left and right (source and drain regions). The lighter shade represents the metal gates. The dot's size is controlled by the middle pair of gates, and its tunnel barriers can be varied by the pairs of gates on the left and on the right.

To observe charge quantization in the dot, two conditions must be satisfied. First, the barriers must be large enough that the transmission is small. This gives the condition $G \ll e^{2} / h$ (i.e., the dot is almost isolated). Second, the temperature must be low enough that the effects of charge quantization are not washed out. The dot's ability to hold charge is described classically by its average capacitance $C$. Since the energy required to add a single electron is $\approx e^{2} / C$ per electron in the dot, we have the condition $k T \ll e^{2} / C$. A typical charging energy of a GaAs disk of radius $0.2 \mu \mathrm{m}$ is $E_{C}=e^{2} / C \sim 1000 \mu \mathrm{eV}$, and the condition $k T \ll e^{2} / C$ is always satisfied at the low temperatures used in experiments. The tunneling of an electron into the dot is usually blocked by the classical Coulomb repulsion of the electrons already in the dot, and the conductance is small. This phenomenon is known as Coulomb blockade. But by changing the gate voltage $V_{g}$ we can compensate for this repulsion, and at the appropriate value of $V_{g}$ the charge on the dot will fluctuate between $\mathcal{N}$ and $\mathcal{N}+1$ electrons, leading to a maximum in the conductance. This leads to so-called Coulomb-blockade oscillations of the conductance as a 
function of the gate voltage. At sufficiently low temperatures these oscillations turn into sharp peaks [see, for example, Fig. 7(c)] that are spaced almost uniformly in $V_{g}$ by an amount essentially proportional to the charging energy $E_{C}$.

(a)
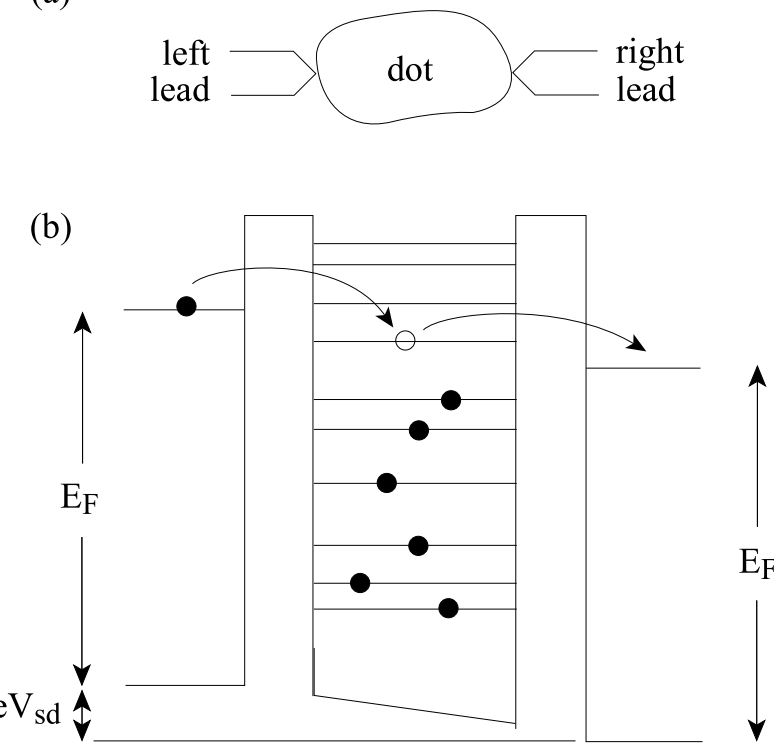

FIG. 2. Conductance by resonant tunneling of a single electron through a quantum dot: (a) Schematic view of an isolated quantum dot that is weakly coupled to left and right leads. The point contacts effectively create tunnel barriers between the dot and the leads. (b) Side view. When the Fermi energy of the electron in the source and drain reservoirs matches the first unoccupied level in the dot, the electron can tunnel across the barrier into the dot. A current will flow in response to a small source-drain voltage $V_{s d}$. The potential in the dot is controlled by the gate voltage $V_{g}$. The effect of Coulomb charging energy is not shown here and is illustrated in Fig. 8.

Coulomb blockade was first observed in tunnel junctions containing a small metallic particle (see, for example, Giaever and Zeller, 1968) in the classical regime ${ }^{1}$ $\Delta \ll k T \ll e^{2} / C$, where tunneling occurs through a large number $(\sim k T / \Delta)$ of levels. Kulik and Shekhter (1975) introduced a transport theory for this classical regime. Early theoretical work on Coulomb blockade effects in a single junction was done by Ben Jacob and Gefen (1985), Likharev and Zorin (1985), and Averin and Likharev (1986). The first controlled experiment on a single-electron tunneling device was by Fulton and Dolan (1987). The first observation of Coulomb block-

\footnotetext{
${ }^{1} \mathrm{~A}$ metallic particle in $3 \mathrm{D}$ has a much smaller mean level spacing than a $2 \mathrm{D}$ dot of the same size because of the differences in dimensionality and effective mass.
}

ade in a semiconductor device was by Scott-Thomas et al. (1989). Low-temperature experiments in semiconductor quantum dots can probe the quantum Coulomb-blockade regime $k T \ll \Delta \ll e^{2} / C$, where tunneling occurs through a single resonance in the dot. Resonant tunneling is illustrated in Fig. 2.

\section{B. From "regular" to chaotic dots}
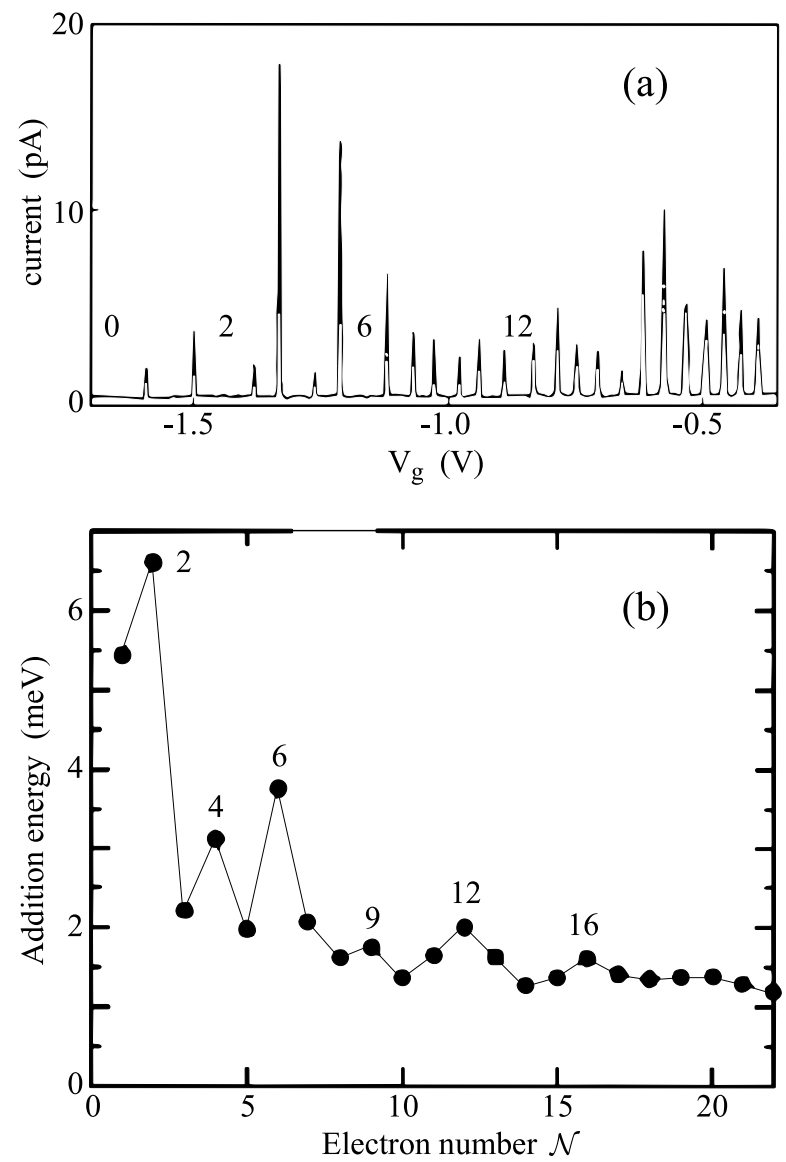

FIG. 3. Shell structure observed in the addition energy of a small vertical quantum dot. The dot has the shape of a $2 \mathrm{D}$ disk with a harmonic-like confining potential. (a) Coulombblockade peaks in the current vs gate voltage $V_{g}$; (b) Addition energy [extracted from the peak spacings shown in panel (a)] as a function of electron number $\mathcal{N}$ in the dot. The maxima correspond to filled $(\mathcal{N}=2,6,12)$ or half-filled $(\mathcal{N}=4,9,16)$ spin-degenerate harmonic-oscillator shells. The half-filling follows Hund's rule favoring the filling of the valence shell with parallel spins. From Tarucha et al. (1996).

Vertical dots are suitable for spectroscopic studies of a dot with few electrons $(\mathcal{N} \lesssim 20)$. Such dots can be prepared in regular shapes, such as a disk, where the confining potential is harmonic and the single-particle levels are arranged in shells. This shell structure is observed by measuring the Coulomb-blockade peaks as a function of 
the number of electrons in the dot (Tarucha et al., 1996; Kouwenhoven, Oosterkamp et al., 1997); see, for example, Fig. 3(a). The spacings between adjacent peaks are not uniform and can be converted into an addition spectrum, shown in Fig. 3(b). The addition spectrum exhibits clear maxima at $\mathcal{N}=2,6$, and 12 , corresponding to completely filled shells of a 2D spin-degenerate harmonic oscillator. Additional maxima can be seen at $\mathcal{N}=4,9$, and 16, describing half-filled shells with parallel spins, in agreement with Hund's rules from atomic physics. The single-particle spectrum is sensitive to a magnetic field (as in atoms), and configuration rearrangements are seen at avoided crossings of single-particle levels. Overall, a simple single-particle model plus constant charging energy, supplemented by a perturbative treatment of the exchange interaction, can explain qualitatively the observed pattern of addition energies versus magnetic field. A good quantitative agreement is obtained when compared with Hartree-Fock calculations of a few-electron system.

Hartree-Fock calculations - feasible in small dots - become impractical for dots with several hundred electrons. Moreover, many of the lateral dots with $\mathcal{N} \gtrsim 50$ electrons often have no particular symmetry. Scattering of an electron from the irregular boundaries of such dots leads to single-particle dynamics that are mostly chaotic. Measured quantities such as the dot's conductance and addition spectrum display "random" fluctuations when various parameters (e.g., shape and magnetic field) are varied. We are entering the statistical regime, in which new kinds of questions are of interest. For example, rather then trying to calculate the precise, observed sequence of conductance peaks in a specific dot, we can study the statistical properties of the dot's conductance sampled from different shapes and applied magnetic fields.

Classical chaos, i.e., the exponential sensitivity of the time evolution of a dynamical system to initial conditions, is well understood not only in closed systems but also in open scattering systems (e.g., quantum dots) assuming that the particle spends sufficient time in the finite scattering regime (e.g., the dot) before exiting into the asymptotic regime (e.g., the leads). In describing transport through coherent systems, we are interested in the quantum manifestations of classical chaos. The link between classical and quantum chaos was first established in 1984 with the Bohigas-Giannoni-Schmit (BGS) conjecture (Bohigas, Giannoni, and Schmit, 1984) that the statistical quantal fluctuations of a classically chaotic system are described by random-matrix theory (RMT). These authors found that the statistical properties of $\sim 700$ eigenvalues of the Sinai billiard - a 2D classically chaotic system - follow the predictions of RMT. Figure 4(b) compares the nearest-neighbor spacing distribution of the Sinai billiard's eigenvalues (histogram) with the same distribution calculated from RMT (solid line).
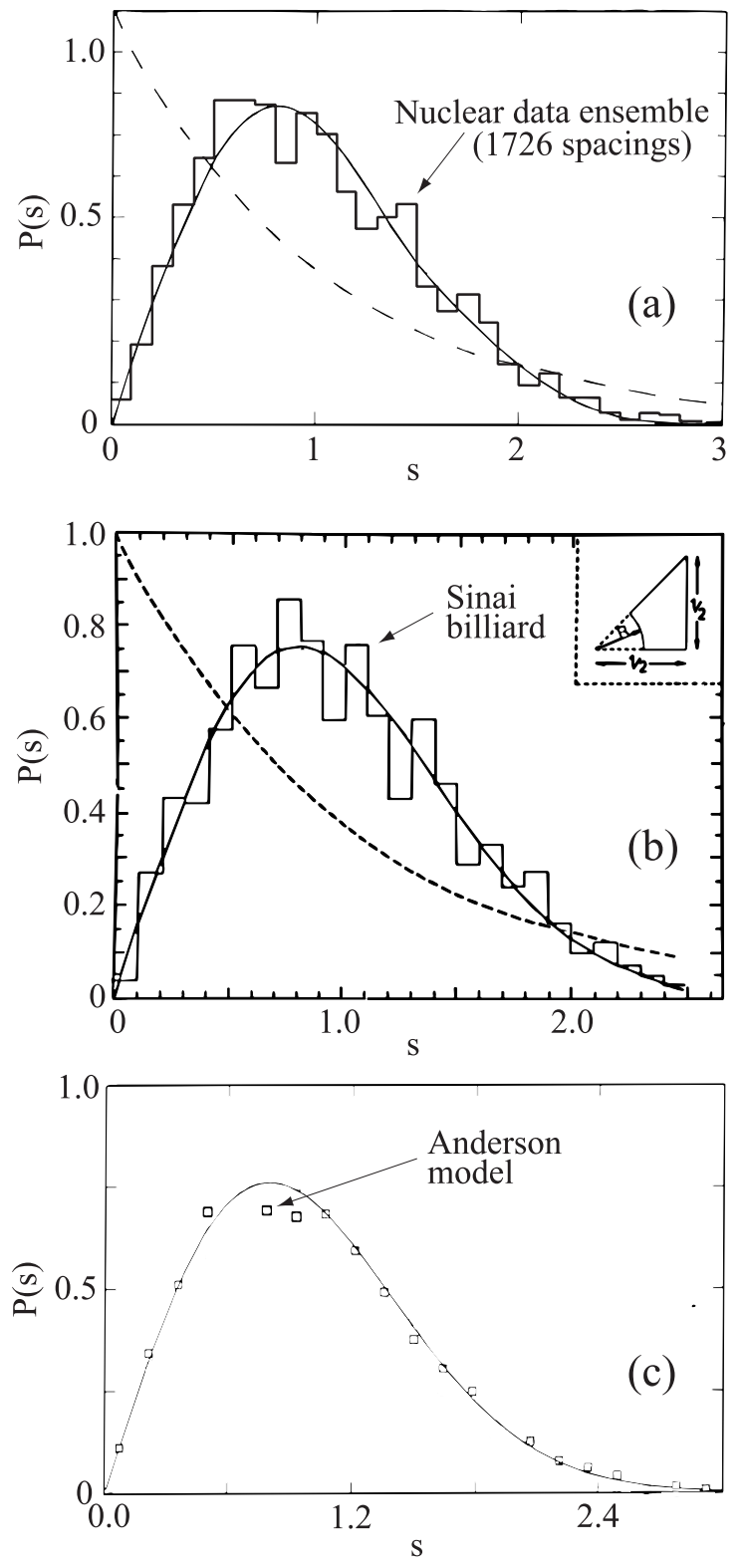

FIG. 4. The universality of RMT. The nearest-neighbor levelspacing distribution $P(s)$ (where $s$ is the spacing in units of the mean level spacing) in (a) a compound nucleus, (b) a 2D chaotic system, and (c) a disordered system, compared with the Wigner-Dyson distribution (solid lines) predicted by RMT. Dashed lines show the Poisson distribution describing $P(s)$ for a random sequence of levels. Panel (a) shows $P(s)$ for the nuclear data ensemble - 1726 neutron and proton resonances measured in several heavy nuclei. From Bohigas, Haq and Pandey (1983). Panel (b) is $P(s)$ for 700 eigenvalues of the Sinai billiard, a classically chaotic system. The eigenfunctions vanish at the boundaries indicated by the inset. From Bohigas, Giannoni, and Schmit (1984). Panel (c) illustrates $P(s)$ for a 3D Anderson model (open squares) in its diffusive regime with on-site disordered potential $w / t=2$ (see Sec. III.A.3). From Dupuis and Montambaux (1991).

Random-matrix theory differs in a fundamental way 
from the conventional statistical approach. Rather than declaring ignorance with respect to the microscopic dynamical state of the system, we declare ignorance with respect to the Hamiltonian itself (Balian, 1968). The only relevant information is the system's fundamental space-time symmetries, and otherwise the Hamiltonian can be chosen "at random." This revolutionary idea was introduced by Wigner in the 1950s to explain the spectral properties of a complex many-body system, the compound nucleus, and was developed by Dyson, Mehta and others in the early 1960s. Since RMT has no scale (its only physical parameter is determined by the mean level spacing $\Delta$ which scales out if all energies are measured in units of $\Delta$ ), it leads to universal predictions. For example, neutron and proton resonances measured in heavy nuclei and collected in the so-called nuclear data ensemble (Bohigas, Haq and Pandey, 1983) were found to obey the predictions of RMT. In particular, the resonances' spacing distribution, represented by the histogram in Fig. 4(a), is well described by the Wigner-Dyson distribution of RMT (solid line), just as the eigenvalues of the Sinai billiard are [Fig. 4(b)].
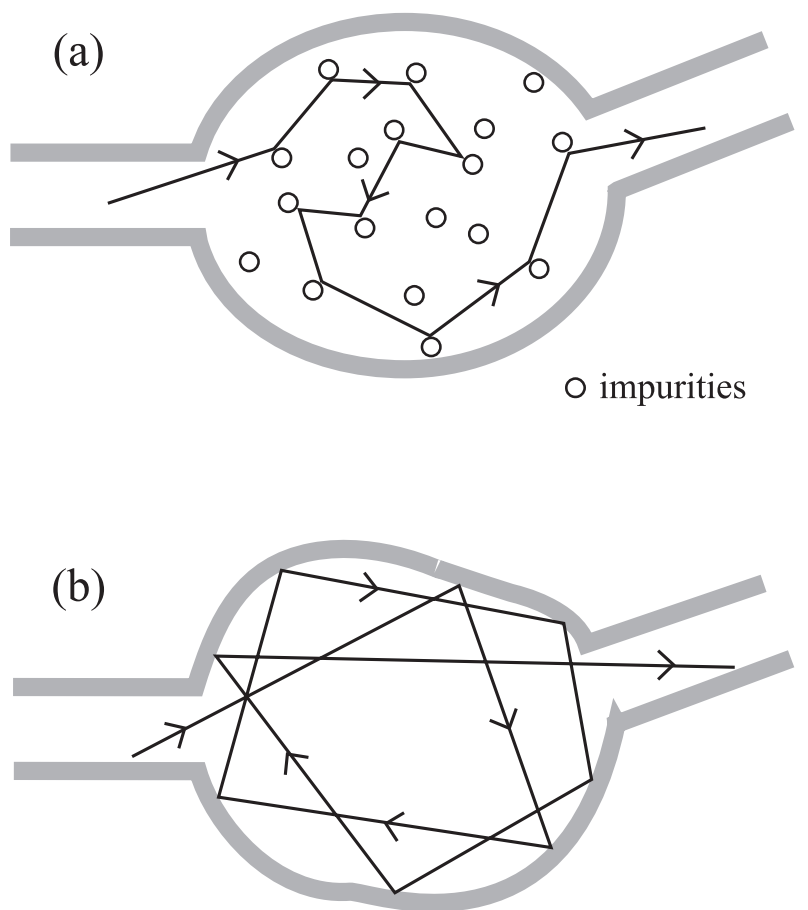

FIG. 5. Schematic drawing of a diffusive dot and a ballistic dot attached to two leads: (a) an impurity-rich diffusive dot, showing an electron's trajectory with elastic scatterings from the impurities; (b) a ballistic dot. There are few impurities and the electron moves ballistically. Typically, an electron is scattered several times from the dot's boundaries before exiting the dot.

The usefulness of RMT for neutron resonances was justified by the complexity of the compound nucleus above the neutron threshold. In contrast, the Sinai billiard is a relatively simple but chaotic $2 \mathrm{D}$ system. According to the BGS conjecture, classical chaos is a sufficient condition for the applicability of RMT. We shall see that RMT is indispensable for understanding the universal statistical properties of chaotic quantum dots. Random-matrix theory also links chaotic ballistic dots to mesoscopic disordered metals, as we discuss next.

\section{From disordered to chaotic ballistic dots}

A disordered, impurity-rich quantum dot - similar to the diffusive systems that were the original focus of mesoscopic physics - is shown schematically in Fig. 5(a). An electron enters the dot through a lead and scatters elastically from the impurities. Here $l \ll L$, and the transport is diffusive ( $l$ is the mean free path and $L$ is the linear size of the dot). The characteristic time scale is $\tau_{D}$, the time for the electron to diffuse across the dot. The associated energy scale is known as the Thouless energy $E_{c}=\hbar / \tau_{D}$. Figure 5(b) illustrates the more common ballistic dot. There is relatively little disorder, and transport is dominated by scattering from the dot's boundaries. When the boundaries are irregular, the electron's dynamics is mostly chaotic. The relevant time scale in ballistic dots is the ergodic time $\tau_{c}$, which is roughly the time of flight across the dot. The related energy scale $E_{T}=\hbar / \tau_{c}$ is termed the ballistic Thouless energy. The chaotic nature of the classical motion inside the dot can be revealed in the conductance only if the electron scatters off the boundaries at least several times before escaping through a lead. We therefore limit our discussion to dots in which $\tau_{\text {escape }} \gg \tau_{c}$, where $\tau_{\text {escape }}$ is the mean escape time of the electron into the leads. Equivalently, the average width $\Gamma$ of a level in the dot (to decay into the leads) must be small compared with $E_{T}\left(\Gamma \ll E_{T}\right)$. For a diffusive dot, a similar condition $\Gamma \ll E_{c}$ is required.

One of the important consequences of quantum coherence is the interference of waves describing an electron propagating along different paths between the incoming and outgoing leads. We can observe these interference effects in the conductance by changing a phasesensitive parameter in the system, for example, the electron's Fermi momentum or the external magnetic field. The conductance through diffusive or ballistic open dots thus exhibits aperiodic but reproducible fluctuations as a function of a parameter [see, for example, Fig. 6(c)].

Early theoretical studies of disordered conductors were based mostly on weak-disorder perturbation theory (i.e., the diagrammatic approach). Two important phenomena were discovered: (i) The average conductance is smaller in the absence of a magnetic field than in its presence, an effect known as weak localization (see Bergmann, 1984, and references therein). This quantum interference effect requires $L_{\phi}>>l$ and occurs already in macroscopic 
conductors $\left(L>L_{\phi}\right)$. (ii) The rms fluctuations of the conductance in a mesoscopic conductor are of the order $e^{2} / h$, independent of the size of the average conductance, a phenomenon known as universal conductance fluctuations (Altshuler, 1985; Lee and Stone, 1985). A natural question that arises is whether similar mesoscopic phenomena can be observed in ballistic dots. Given the BGS conjecture, the application of RMT to describe the universal features of the conductance through chaotic ballistic dots was a logical step. As long as the electron dynamics in the dot are chaotic, the conductance fluctuations are independent of the dot's geometry, although they do depend on the properties of the leads (such as the number of modes and the average transparency of each mode). Only in the limit of a large number of fully transmitting modes does the size of the fluctuations become truly universal in the sense of universal conductance fluctuations.

The link between universal conductance fluctuations in disordered systems and RMT was first postulated by Altshuler and Shklovskii (1986) and by Imry (1986a). Earlier work by Thouless had pointed to the close connection between the conductance and spectral properties: the conductance expresses the sensitivity of the energy levels to a change in the boundary conditions (Edwards and Thouless, 1972; Thouless, 1974, 1977). This relation was used by Altshuler and Shklovskii (1986) to argue that RMT spectral correlations are at the origin of universal conductance fluctuations. Their diagrammatic calculations also showed that spectral correlations in disordered systems are nonuniversal for energy scales that exceed the Thouless energy. At such energy scales the electron diffuses for times that are short compared with $\tau_{D}$ and therefore does not have enough time to reach the system's boundaries. An analogous breaking of RMT universality in chaotic systems was demonstrated by Berry (1985) for time scales that are shorter than the ergodic time. These observations suggested that the universal regime of RMT is applicable to energy scales that are smaller than the Thouless energy in disordered systems, and smaller than the ballistic Thouless energy in ballistic chaotic systems.

A proof of RMT universality in the disordered case was achieved using the supersymmetry method, a fieldtheoretical approach to disordered systems (Efetov, 1983). The supersymmetry method is a technique to carry out the ensemble average of a product of Green's functions, where the original disordered problem is mapped onto a supersymmetric nonlinear $\sigma$ model. Below the Thouless energy this field-theoretical model reduces to $0 \mathrm{D}$, where it is equivalent to RMT. The RMT universality in weakly disordered systems is demonstrated in Fig. 4(c), where the nearest-neighbor levelspacing distribution, calculated numerically for a disordered metal, is seen to follow the Wigner-Dyson distribution of RMT (solid line).

\section{From open to closed dots}

The strength of the dot-lead coupling affects the width of a typical resonance in the dot to decay into the leads. A simple expression for the width can be obtained from an argument due to Weisskopf (1937). Imagine a wave packet near the entrance to a channel $c$ in one of the leads. The wave packet evolves in time and returns after the recurrence time or Heisenberg time $\tau_{H}=h / \Delta$, where $\Delta$ is the mean level spacing. ${ }^{2}$ The probability to decay if close to a channel $c$ is given by the transmission coefficient $T_{c}$. The decay rate $\Gamma_{c} / \hbar$ of a level into channel $c$ is then given by the frequency of attempted decays times the transmission coefficient (i.e., $\tau_{H}^{-1} T_{c}$ ), and the total width $\Gamma=\sum_{c} \Gamma_{c}$ of a level to decay into any of the channels is

$$
\Gamma=\frac{\Delta}{2 \pi} \sum_{c} T_{c}
$$

In an open dot, the width $\mathcal{W}$ of the dot-lead interface is much larger than the Fermi wavelength, so that the lead supports a large number of open modes $\left(\Lambda=\operatorname{Int}\left[k_{F} \mathcal{W} / \pi\right] \gg 1\right)$ with transmission coefficients of order $\sim 1$ (no tunnel barriers). From Eq. (1) we immediately see that $\Gamma \gg \Delta$. Thus in open dots the resonances are strongly overlapping. This is analogous to the compound-nucleus regime of many overlapping resonances, which occurs at several $\mathrm{MeV}$ above the neutron threshold. Ericson $(1960,1963)$ predicted that in this regime the coherent superposition of a large number $(\sim \Gamma / \Delta)$ of resonance amplitudes would cause "random" but reproducible fluctuations of the nuclearreaction cross section as a function of the reaction energy. Ericson fluctuations were observed a few years after his prediction in light-ion reactions (Ericson and MayerKuckuk, 1966). An example is shown in Fig. 6(a), where the measured differential cross section for the reaction $p$ $+{ }^{35} \mathrm{Cl} \rightarrow \alpha+{ }^{32} \mathrm{~S}$ is plotted as a function of the proton energy. Ericson argued that the energy autocorrelation function of the cross section should be a Lorentzian with a width $\Gamma$ that is just the width of a typical resonance in the nucleus. This is shown in Fig. 6(b), where the autocorrelation function of the cross section (solid line) is fitted to a Lorentzian (dashed line). Similar fluctuations were observed in the conductance $G$ of a ballistic open dot as a function of the Fermi momentum $\hbar k_{F}$, as shown in Fig. 6(c). A convenient way of analyzing the fluctu-

\footnotetext{
${ }^{2}$ In the original Weisskopf argument, $\Delta$ is the many-body mean level spacing. However, we are mostly interested in the immediate vicinity of the ground state of the dot where (for not-too-strong interactions) the many-particle mean level spacing is of the order of the single-particle level spacing.
} 
ations is to consider the Fourier transform of the autocorrelation function of the conductance, i.e., the power spectrum $S\left(f_{k}\right)$ of $G=G\left(k_{F}\right)$. If the autocorrelation of the conductance vs energy is a Lorentzian of width $\Gamma$, then $S\left(f_{k}\right) \propto e^{-2 \pi \Gamma\left|f_{k}\right| / \hbar v_{F}}$ (where $v_{F}$ is the Fermi velocity). This exponential behavior is demonstrated in Fig. $6(\mathrm{~d})$ for $S\left(f_{V}\right)$, the power spectrum of the conductance vs gate voltage (changing the gate voltage is equivalent to changing the Fermi energy). For ballistic dots we can give a semiclassical interpretation of $\Gamma$ : in an open chaotic system the classical escape time is distributed exponentially, with a characteristic mean escape time of $\tau_{\text {escape }}=\hbar / \Gamma$.
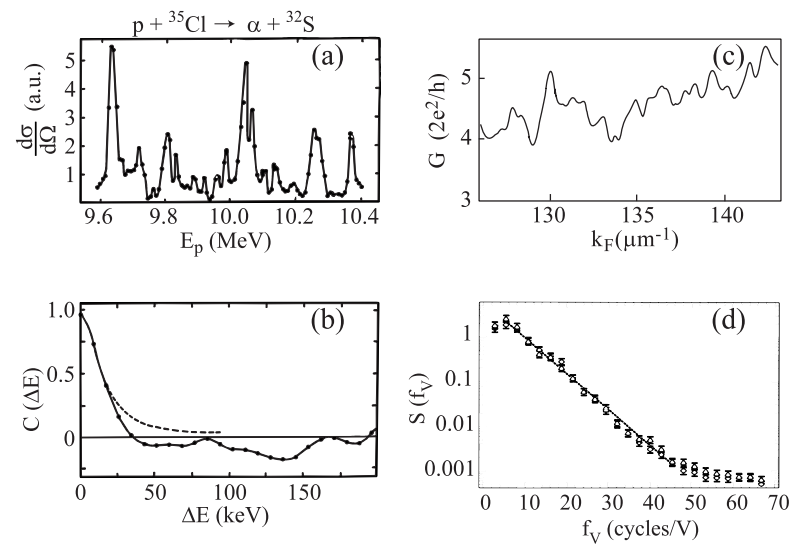

FIG. 6. Ericson fluctuations in the compound nucleus [panels (a) and (b)] and in an open quantum dot [panels (c) and (d)]: (a) the measured differential cross section $d \sigma / d \Omega$ at $\theta=170^{\circ}$ for the reaction $p+{ }^{35} \mathrm{Cl} \rightarrow \alpha+{ }^{32} \mathrm{~S}$ vs the incoming proton energy $E_{p}$. This reaction proceeds through the compound nucleus ${ }^{36} \mathrm{Ar}$ at an excitation energy of $\sim 18.5 \mathrm{MeV}$; (b) the energy autocorrelation function $C(\Delta E)$ (solid line) of the cross section in panel (a) (von Brentano et al., 1964), where the dashed line is a Lorentzian fit whose width $\Gamma$ corresponds to the lifetime $\hbar / \Gamma$ of the excited compound nucleus ${ }^{36} \mathrm{Ar}$ (from Ericson and Mayer-Kuckuk, 1966); (c) the conductance $G$ measured in an open chaotic dot as a function of the electron's Fermi momentum $k_{F}$ (Keller et al., 1996); the average conductance increases as a function of $k_{F}$, but the magnitude of the fluctuations is constant of the order $e^{2} / h$; (d) the power spectrum $S\left(f_{V}\right)$ (Chang et al., 1995), i.e., the Fourier transform of the conductance autocorrelation function vs gate voltage (changing the gate voltage is equivalent to changing the Fermi energy). The dashed line is a fit to an exponential (which corresponds to a Lorentzian form of the conductance autocorrelation function). A log-linear scale is used.

The cross section of a nuclear reaction is proportional to the squared $S$-matrix element between the entrance and exit channels. An analogous situation exists in coherent transport through open quantum dots. The formulation of conductance in coherent systems was pioneered by Landauer $(1957,1970)$ and refined by Imry (1986b) and Büttiker (1986a). They described the con- ductance as a scattering process and expressed it directly in terms of the total transmission through the sample. ${ }^{3}$ The total transmission is the sum over squared $S$-matrix elements between all entrance and exit channels. The average conductance is then expected to increase linearly with the number of open channels, as can be seen in Fig. 6(c). However, the magnitude of the fluctuations is $\sim e^{2} / h$, independent of the average conductance. The nuclear cross-section fluctuations are also universal, although since the measured cross section corresponds to a specific selection of exit and entrance channels, the size of the fluctuations is comparable to the average.

In recent years the experimental focus has shifted from open to closed dots, where the statistical behavior of individual wave functions can be probed. In closed dots the transmission coefficients are small, $T_{c} \ll 1$, and according to Eq. (1), $\Gamma \ll \Delta$ (assuming a small number of channels). This is the regime of isolated resonances, analogous to the compound-nucleus regime of isolated neutron resonances just above the neutron threshold. Such narrow resonances were observed in the total cross section to scatter thermal neutrons from heavy nuclei. Figure 7(a) shows such resonances for the reaction $n+{ }^{232} \mathrm{Th}$. The distribution of the widths of these resonances is shown on a log-linear scale in Fig. 7(b) and is well described by the so-called Porter-Thomas distribution (solid line) predicted by RMT. It is given by $P(\hat{\Gamma}) \propto \hat{\Gamma}^{-1 / 2} e^{-\hat{\Gamma} / 2}$, where $\hat{\Gamma}$ is the width measured in units of the average width.

In closed dots the conductance is not a smooth function of the gate voltage as in open dots, but instead exhibits Coulomb-blockade peaks [see, for example, Fig. 7(c)]. The spacings between these peaks are almost uniform because they are dominated by the large charging energy, in contrast to the nuclear case, where the spacings between the observed resonances fluctuate widely. Moreover, the observed conductance peak widths in closed dots are all $\sim k T$ because of thermal broadening. However, the peak heights exhibit order-of-magnitude fluctuations, as can be seen in Fig. $7(\mathrm{c})$. These peak fluctuations are determined by the spatial fluctuations of the individual resonance wave functions in the vicinity of the leads. The statistical approach to Coulomb-blockade peak heights was developed by Jalabert, Stone, and Alhassid (1992). They used $R$-matrix theory - originally introduced by Wigner and Eisenbud (1947) for nuclear reactions - to relate the Hamiltonian of the closed system to the scattering resonances of the weakly open system, and then

\footnotetext{
${ }^{3}$ It is interesting to note that Landauer's formula can be derived from Weisskopf's formula (1) by applying the latter to the leads instead of the dot (where the leads are considered as decaying quantum systems emitting electrons); see Bertsch (1991).
} 
applied an RMT approach to quantify the wave-function fluctuations. The conductance peak-height distributions were found to be universal and sensitive only to the space-time symmetries of the dot. These distributions were measured a few years later (Chang et al., 1996; Folk et al., 1996) and were in agreement with the theoretical predictions. The distribution of the conductance peak heights in the absence of magnetic field is shown in Fig. 7(d). This is the case of conserved time-reversal symmetry, analogous to the neutron-resonance statistics in Fig. 7(b).
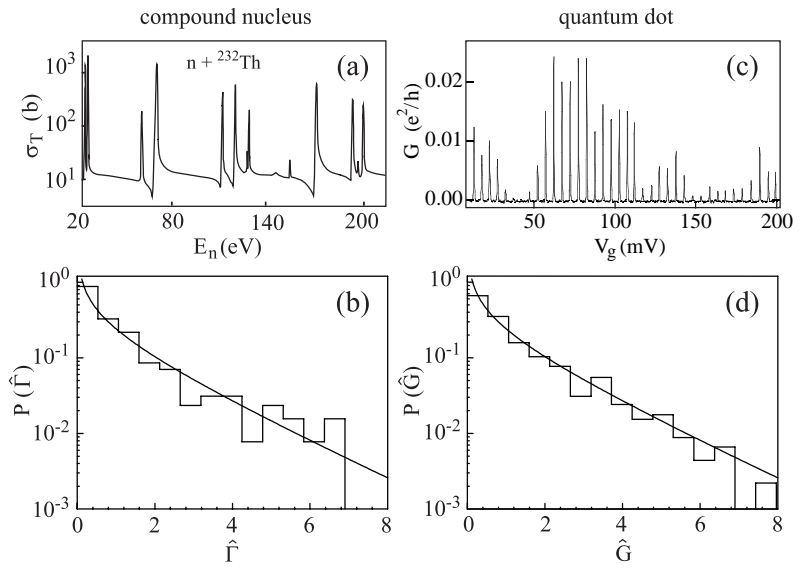

FIG. 7. Neutron-resonance-width statistics in the compound nucleus [panels (a) and (b)] and Coulomb-blockade peak statistics in closed quantum dots [panels (c) and (d)]: (a) a series of neutron resonances in the total cross section $\sigma_{T}$ (in barns) of $\mathrm{n}+{ }^{232} \mathrm{Th}$ as a function of the incoming neutron energy $E_{n}$ (in eV) (from Brookhaven National Laboratory, 1964); (b) distribution of the normalized neutron resonance widths $\hat{\Gamma}=\Gamma / \bar{\Gamma}$ using a log-linear scale $[223$ neutron resonances in ${ }^{233} \mathrm{Th}$ are included from the measurements of Garg et al. (1964)]; the solid line is the Porter-Thomas distribution $P(\hat{\Gamma}) \propto \hat{\Gamma}^{-1 / 2} e^{-\bar{\Gamma} / 2}$ predicted by RMT; (c) a series of Coulomb-blockade peaks observed in the conductance $G$ of closed GaAs/AlGaAs dots (with $\mathcal{N} \sim 1000$ electrons) as a function of gate voltage $V_{g}$ at zero magnetic field (Folk et al., 1996); (d) distribution of the normalized conductance peak heights $\hat{G}=G / \bar{G}$ (histogram) on a log-linear scale; 600 peaks are included, of which only $\sim 90$ are statistically independent. The solid line is the theoretical prediction based on RMT (Jalabert, Stone and Alhassid, 1992) and contains no free parameters. Notice the agreement with the experiment over almost three orders of magnitude.

\section{E. From noninteracting to interacting electrons}

Studies of statistical fluctuations in open dots have generally ignored electron-electron interactions. Electron-electron inelastic scattering reduces the coherence time of the electrons at finite temperature, but otherwise the excitations around the Fermi energy are assumed to be noninteracting quasiparticles. Landau's
Fermi-liquid theory treats the electrons in good metals as weakly interacting quasiparticles whose lifetime near the Fermi surface is large compared to $\hbar / k T$. In open dots with many open channels the average conductance is large, and a noninteracting picture, similar to that of metals, is justified. But as the strength of the dot-lead couplings is reduced, interaction effects become important in transport. The simplest way to take interactions into account is to include only the longrange component of the Coulomb interaction, namely the average interaction among $\mathcal{N}$ electrons in the dot. This is known as the constant-interaction model. This model has the advantage of simplicity, and it does explain some of the observed phenomena. However, discrepancies with other recent experiments - notably the measured peak-spacing distribution - indicate that electronelectron interactions can have significant effects beyond the constant-interaction model. An important parameter is the dimensionless gas parameter $r_{s}$ measuring the ratio between a typical Coulomb interaction and the average kinetic energy. When $r_{s}$ is small, it is possible to use a combination of mean-field approximations and the known statistics of the noninteracting limit. However, in semiconductor quantum dots, $r_{s} \sim 1-2$, and deviations are expected. The interplay between one-body chaos and many-body interactions in quantum dots is a fascinating open problem and is currently the main focus of the field.

\section{F. Methods}

The statistical theory of quantum dots is based on four main approaches: diagrammatic methods, semiclassical methods, random-matrix theory, and supersymmetry. The first approach (i.e., the diagrammatic method) was developed in early work on disordered conductors. More recent progress - based primarily on the last three methods and driven in part by a deeper understanding of their interrelations - has brought the statistical approach to a new level of maturity. While several reviews and books have been written on the semiclassical, RMT, and supersymmetry approaches, they are usually discussed separately and often not in relation to quantum dots. A section of this review describes these methods in the context of the statistical theory of quantum dots, emphasizing the relationships among the methods. Many of the results of that section will be used in subsequent sections.

\section{G. Outline of the review}

We begin by reviewing transport theory in quantum dots (Sec. II). An important result is the Landauer formula expressing the conductance in terms of the $S$ matrix of the device. We discuss resonant tunneling 
and Coulomb blockade in closed dots, and processes that dominate the off-resonance conductance, such as cotunneling. The results of this section will be used in developing the statistical theory of quantum dots.

Section III covers the main principles and tools of the statistical fluctuation theory: the semiclassical approach (demonstrated for disordered metals), randommatrix theory, and the supersymmetry method.

Section IV examines mesoscopic conductance fluctuations in open dots using random-matrix and semiclassical approaches. We discuss the main mesoscopic phenomena in fully coherent open quantum dots - weak localization and conductance fluctuations, and how they are affected by a finite coherence time.

Section V reviews the statistical theory of closed dots. The statistics and parametric correlations of the peak heights are derived both for temperatures that are small and that are comparable to the mean level spacing. The constant-interaction model can explain some, but not all, of the observed statistics. Adding electrons to the dot changes or "scrambles" the single-particle spectrum and wave functions because of charge rearrangement. This statistical scrambling is discussed in the framework of RMT.

Section VI summarizes recent progress in understanding the effects of electron-electron interactions on the statistical fluctuations. The focus is on peak-spacing statistics, where discrepancies with the results of the CI model have been seen experimentally. The Hartree-Fock approximation offers an intermediate framework that describes interaction effects within a single-particle theory. Spin effects are also discussed.

Finally, in Sec. VII we consider various charging energy effects on the mesoscopic fluctuations, such as the fluctuations of the off-resonance conductance where the dominating conductance mechanism is cotunneling, and the phenomenon of mesoscopic Coulomb blockade due to the backscattering of electrons into an open lead.

Throughout the review we have made an effort to adhere to a uniform notation, which sometimes required changing the notation of the original papers. We have also tried to keep to standard notation except in cases where the same symbol is used for different quantities. The topics covered in this review are too broad to permit discussion of all related work, and we apologize to those whose work could not be included.

\section{ELECTRON TRANSPORT THROUGH QUANTUM DOTS}

Conductance through quantum dots is a phasecoherent process. In this section we review the formalism of coherent transport.

In Sec. II.A we remind the reader of the quasiclassical theory of transport in a metal where phase coherence is ignored. At low temperatures and in small conductors, however, the coherence length of the electron can be larger than the system's size, and conductivity is no longer a local quantity. Section II.B describes conductance as a coherent scattering process; its main result is the Landauer formula expressing the conductance in terms of the $S$ matrix. It is often useful to relate the $S$ matrix of the open system to the eigenfunctions and eigenvalues of the closed system. This relation is derived in $R$-matrix theory, discussed in Sec. II.C. Section II.D describes an important limit of the theory, the weak-coupling limit, where conductance occurs by resonant tunneling.

Section II.E reviews Coulomb blockade, a central phenomenon in closed quantum dots with large tunnel barriers. Coulomb blockade is essentially a classical phenomenon, observed at temperatures that are small compared with the charging energy. The discreteness of the dot's levels becomes important in the quantum Coulombblockade regime when the temperature drops below the mean level spacing $\Delta$.

Section II.F considers the off-resonance conductance in Coulomb-blockade dots, which is dominated by cotunneling, i.e., the virtual tunneling of an electron (hole) through a large number of intermediate levels in the dot. Finally, Sec. II.G explains how conductance measurements in the nonlinear regime provide information on excited states in the dot.

\section{A. Quasiclassical description of conductivity}

In a perfect periodic potential in a crystal, the motion of the electrons is described by extended Bloch states. In real samples, however, there is some disordered potential due to impurities, defects, dislocations, etc. The electrons scatter from the impurities elastically (i.e., the electron's energy is conserved and only its momentum is reoriented), and repeated scatterings lead to diffusive motion. In the quasiclassical description of diffusion, the electron is assumed to lose phase coherence after each collision with an impurity, i.e., $L_{\phi} \sim l$, and the conductivity can be introduced as a local intensive quantity (see below).

The diffusion current of electrons is given by $\boldsymbol{J}_{d}=$ $-D \nabla n_{s}$, where $n_{s}=n_{s}(\boldsymbol{r}, t)$ is the electron density and $D$ is the diffusion constant. Using the continuity equation $\nabla \cdot \boldsymbol{J}_{d}=-\partial n_{s} / \partial t$, we obtain the diffusion equation

$$
\frac{\partial n_{s}}{\partial t}=D \nabla^{2} n_{s} .
$$

Describing the diffusion (in $d$ dimensions) as a random walk, we can relate the diffusion constant $D$ to the elastic mean free path $l$ through $D=v_{F} l / d$.

Special solutions to the diffusion equation, $n_{\boldsymbol{q}} \propto$ $e^{i \boldsymbol{q} \cdot \boldsymbol{r}} e^{-D q^{2} t}$, are known as the classical diffusion modes. 
The classical diffusion propagator, describing the time evolution of the single-electron density, can be expanded in these modes: $D\left(\boldsymbol{r}^{\prime}, \boldsymbol{r} ; t\right)=\left[\sum_{\boldsymbol{q}} e^{i \boldsymbol{q} \cdot\left(\boldsymbol{r}^{\prime}-\boldsymbol{r}\right)} e^{-D q^{2} t}\right] \theta(t)$. Its Fourier transform is $D(\boldsymbol{q}, \omega)=1 /\left(-i \omega+D q^{2}\right)$.

The electrical conductivity $\sigma$ is defined by Ohm's law, $\boldsymbol{J}_{e}=\sigma \boldsymbol{E}$, describing the linear relation between the electric current density $\boldsymbol{J}_{e}$ and the applied electric field $\boldsymbol{E}$. To relate the conductivity to the mean free time $\tau$ $\left(\tau=l / v_{F}\right.$, where $v_{F}$ is the Fermi velocity), we assume that after each collision the electron's velocity is "randomized." Between collisions the electrons are accelerated in response to the electric field, and their average velocity is $\overline{\boldsymbol{v}}=(e / m) \boldsymbol{E} \tau$. Since the current is given by $\boldsymbol{J}_{e}=n_{s} e \overline{\boldsymbol{v}}$, we recover Ohm's relation with

$$
\sigma=\frac{e^{2}}{m} n_{s} \tau
$$

also known as Drude's formula. When combined with $D=v_{F}^{2} \tau / d$ and $d n_{s} / 2 E_{F}=\nu$ ( $\nu$ is the density of singleparticle states per unit volume at the Fermi energy), Drude's formula (3) leads to Einstein's relation

$$
\sigma=e^{2} \nu D
$$

In a $2 \mathrm{D}$ electron gas, $\nu=m / \pi \hbar^{2}$ is constant, and $\sigma=$ $\frac{e^{2}}{h} k_{F} l$. In a good metal, $k_{F} l \gg 1$, and the conductivity is much larger than the quantum unit $e^{2} / h$.

The quantity that is measured directly is the conductance, defined as the ratio between the current and the applied voltage. In a macroscopic conductor the conductivity is an intensive quantity, and the conductance $G$ is related to the conductivity by $G \sim \sigma(\mathcal{S} / L)$, where $L$ and $\mathcal{S}$ are the length and transverse cross section of the conductor, respectively.

\section{B. Conductance in mesoscopic systems and the Landauer formula}

In a mesoscopic system $L_{\phi} \gtrsim L$, and the local description of conductivity breaks down. It is then meaningful to discuss only the measurable quantity - the conductance - or alternatively, to introduce conductivity as a nonlocal tensor. For recent books discussing transport in mesoscopic systems, see, for example, Datta (1995), Imry (1996), and Ferry and Goodnick (1997).

The transport properties of a mesoscopic structure are characterized by conductance coefficients that are sample specific. Several probes (leads) attached to electron reservoirs are connected to the system. A current is passed through the structure and the voltage is measured at the different probes. Denoting by $V_{n}$ the voltage of probe $n$ and by $I_{n}$ the current through probe $n$, we expect in the limit of small voltages a set of linear relations

$$
I_{n^{\prime}}=\sum_{n} G_{n^{\prime} n} V_{n}
$$

The coefficient $G_{n^{\prime} n}$ is the conductance between leads $n$ and $n^{\prime}$. It follows from Kirchhoff's law $\sum_{n} I_{n}=0$ that $\sum_{n} G_{n^{\prime} n}=\sum_{n^{\prime}} G_{n^{\prime} n}=0$. For the special case of a two-lead dot, $G_{12}=G_{21}=-G_{11}=-G_{22} \equiv G$, and $G=I_{1} /\left(V_{2}-V_{1}\right)$.

A formulation that takes into account phase coherence as well as the geometry of the structure was developed by Landauer $(1957,1970)$ and Imry (1986b). The basic idea was to relate the conductance to an underlying scattering matrix (i.e., transmission coefficients). The first derivations of such transmission formulae from linearresponse theory were by Economou and Soukoulis (1981) and Fisher and Lee (1981). The work of Landauer and Imry was generalized by Büttiker (1986a, 1988a) to a general configuration of probes. We first discuss the macroscopic approach of Büttiker and then describe a simplified derivation of the Landauer formula from statistical reaction theory by Bertsch (1991). For illuminating discussions of the Landauer approach and its applications, see Stone and Szafer (1988), Baranger and Stone (1989), Datta (1995), Stone (1995), and Imry (1996). For a recent review, see Imry and Landauer (1999).

We assume a mesoscopic structure connected to several leads $n$, each supporting $\Lambda_{n}$ propagation modes of the electrons. We denote by $S_{c^{\prime} c}^{n^{\prime} n}$ the scattering amplitude of the structure to scatter an electron from channel $c$ in lead $n$ to channel $c^{\prime}$ in lead $n^{\prime}$. A scattering amplitude between different leads $\left(n^{\prime} \neq n\right)$ is called a transmission amplitude $t$, while a scattering amplitude between channels that belong to the same lead $\left(n^{\prime}=n\right)$ is called a reflection amplitude $r$. The total transmission from lead $n$ to lead $n^{\prime}$ is $T^{n^{\prime} n}=\sum_{c^{\prime} c}\left|t_{c^{\prime} c}^{n^{\prime} n}\right|^{2}$ (where the sum is over all channels $c$ in lead $n$ and $c^{\prime}$ in lead $n^{\prime}$ ), and the total reflection in lead $n$ is $R^{n n}=\sum_{c^{\prime} c}\left|r_{c^{\prime} c}^{n n}\right|^{2}\left(c\right.$ and $c^{\prime}$ are channels in lead $n$ ). A voltage $V_{n}$ in probe $n$ causes a current $I_{c n}^{\text {inject }}=e v_{c}\left(d n_{c}^{+} / d \epsilon\right) e V_{n}$ to be injected in each mode $c$, where $v_{c}$ and $d n_{c}^{+} / d \epsilon$ are the longitudinal velocity and density of ingoing states (per unit length of the lead) in mode $c$. Assuming noninteracting electrons in the lead, $v_{c}\left(d n_{c}^{+} / d \epsilon\right)=1 / h$ and the injected current is $I_{c n}^{\text {inject }}=\left(e^{2} / h\right) V_{n}$. The electrons injected in lead $n$ are scattered into lead $n^{\prime} \neq n$ and produce there a current $I_{n^{\prime} n}=\sum_{c^{\prime} c}\left|t_{c^{\prime} c}^{n^{\prime} n}\right|^{2} I_{c n}^{\text {inject }}=\left(e^{2} / h\right) T^{n^{\prime} n} V_{n}$. Electrons are also backscattered into the same lead $n$ giving a net current of $I_{n n}=\left(e^{2} / h\right)\left(R^{n n}-\Lambda_{n}\right) V_{n}$, where the injected current has been subtracted. The total current in lead $n^{\prime}$ is $I_{n^{\prime}}=\sum_{n} I_{n^{\prime} n}$. We obtain Eq. (5) with

$$
G_{n^{\prime} n}=\left\{\begin{array}{ll}
2 \frac{e^{2}}{h} T^{n^{\prime} n} & \left(\text { for } n^{\prime} \neq n\right) \\
2 \frac{e^{2}}{h}\left(R^{n n}-\Lambda_{n}\right) & \left(\text { for } n^{\prime}=n\right)
\end{array},\right.
$$

where a factor of 2 was included to take into account the spin degeneracy of the electrons.

A simple derivation of Landauer's formula at finite temperature is provided by Bertsch (1991). The starting point is Weisskopf's formula (1), first applied in nu- 
clear physics to neutron evaporation (Weisskopf, 1937), and often used in the transition state theory of chemical reactions. We assume for simplicity that the mesoscopic structure is connected to two leads only. Rather than applying Eq. (1) to the structure itself, we apply it to the leads, considering each lead as an equilibrated, decaying system emitting electrons. At zero temperature, the decay rate $\Gamma / \hbar$ from each lead is given by

$$
\frac{\Gamma}{\hbar}=\frac{1}{2 \pi \hbar \rho(E)} \sum T
$$

where $\rho(E)$ is the density of states in that lead and $\sum T$ represents a sum over transmission coefficients in all open channel states. At finite temperature the current due to electrons emitted by the lead is $I=$ $e \sum_{\lambda}\left(\Gamma\left(E_{\lambda}\right) / \hbar\right) f\left(E_{\lambda}-\mu\right)$, where $f\left(E_{\lambda}-\mu\right)$ are FermiDirac occupation probabilities ( $\mu$ is the chemical potential in the lead). Converting the sum into an integral $\sum_{\lambda} \rightarrow \int d E \rho(E)$ and using Eq. (7), we find that the level density cancels out and we obtain

$$
I=\frac{e}{2 \pi \hbar} \int d E\left(\sum T\right) f(E-\mu)
$$

To find the conductance we apply a small source-drain voltage $V_{s d}$ and calculate the current. Each lead emits a current given by Eq. (8). However, the two leads have different chemical potentials $\mu_{1}$ and $\mu_{2}$, with $\mu_{1}-\mu_{2}=$ $e V_{s d}$. The net current $I=I_{1}-I_{2}$ is thus nonzero:

$$
I=\frac{e}{2 \pi \hbar} \int d E\left(\sum T\right)\left[f\left(E-\mu_{1}\right)-f\left(E-\mu_{2}\right)\right]
$$

where we have assumed that the total transmission $\sum T$ is independent of the direction of flow of the current. Indeed, for $V_{s d}=0$ the whole system is equilibrated $\left(\mu_{1}=\mu_{2}\right)$ and the net current must be zero, leading to the equality of $\sum T$ for both leads. In the linear regime, $f\left(E-\mu_{1}\right)-f\left(E-\mu_{2}\right) \approx-e V_{s d} f^{\prime}\left(E-E_{F}\right)$, where $E_{F}$ is the finite-temperature Fermi energy (i.e., chemical potential) of the equilibrated system, and we find

$$
G=2 \frac{e^{2}}{h} \int d E\left[-f^{\prime}\left(E-E_{F}\right)\right]\left(\sum T\right) .
$$

The factor of 2 accounts for spin degeneracy. Equation (10) is the finite-temperature Landauer formula. According to transition state theory, $\sum T$ represents a sum over all available channels. Since the electron can decay from any mode $c$ in the first lead to any mode $c^{\prime}$ in the second lead, $\sum T=T^{2,1}$, where $T^{2,1}$ is the total transmission between the two leads. In the limit of zero temperature, $-f^{\prime}\left(E-E_{F}\right) \rightarrow \delta\left(E-E_{F}\right)$, and Eq. (10) reduces to the first case of Eq. (6).

A more microscopic derivation of Landauer's formula using linear-response theory is based on a Kubo formula (Kubo, 1957; Greenwood, 1958); see, for example,
Baranger and Stone (1989). The generalization of Landauer's formula (10) to the multileads case is

$$
G_{n^{\prime} n}=2 \frac{e^{2}}{h} \int_{0}^{\infty} d E\left[-f^{\prime}\left(E-E_{F}\right)\right] \sum_{c^{\prime} c}\left|S_{c^{\prime} c}^{n^{\prime} n}(E)\right|^{2}
$$

where the sum is over all entrance channels in lead $n$ and all exit channels in lead $n^{\prime}$.

The Landauer formula works well in open structures where the picture of noninteracting quasiparticles is valid and interactions contribute only through finite dephasing times. In closed dots, where charging energy is important, it usually does not hold except in special cases (see Sec. II.E). There were attempts to generalize Landauer's formula to the interacting case. Meir and Wingreen (1992) used the nonequilibrium Keldish formalism to derive a formula for the current in an interacting-electron region. At the limit of zero temperature, where only elastic processes are allowed, their formula reduces for the linear-response conductance reduces to a Landauertype formula. However, at finite temperature or in the nonlinear regime, such Landauer-type formula does not hold because of inelastic processes.

The symmetry properties of the conductance coefficients were discussed by Büttiker (1988a). The timereversal properties of the $S$ matrix in an external magnetic field $B, S^{T}(-B)=S(B)$, lead to $G_{n^{\prime} n}(B)=$ $G_{n n^{\prime}}(-B)$. These relations do not imply that $G$ is a symmetric matrix except when $B=0$. Note, however, that for a two-lead $\operatorname{dot} G$ is always symmetric [see the discussion following Eq. (5)].

\section{R-matrix formalism}

$R$-matrix theory relates the $S$ matrix of the dot to the discrete eigenvalues and eigenstates of the closed system, and was introduced by Wigner and Eisenbud (1947) in nuclear-reaction theory. The methods were generalized and reviewed by Lane and Thomas (1958), and a pedagogical treatment is available in Blatt and Weisskopf (1952). An equivalent formulation that expresses the $S$ matrix in terms of an effective non-Hermitian Hamiltonian of the open system was presented by Mahaux and Weidenmüller (1969) and is also derived in a recent review on scattering in chaotic systems by Fyodorov and Sommers (1997). The $R$-matrix formalism was adapted to quantum dots by Jalabert, Stone, and Alhassid (1992).

We consider a $2 \mathrm{D}$ cavity in the region $\mathcal{A}$ of the $x-y$ plane and assume left and right leads (along the $x$ axis) that are attached to the dot at the lines of contact $x=$ $x_{l}, x_{r}$ (denoted by $\mathcal{C}_{l}$ and $\mathcal{C}_{r}$ ). We denote by $H$ the dot's Hamiltonian and consider the eigenvalue problem

$$
H \Psi_{\lambda}=E_{\lambda} \Psi_{\lambda}
$$


where $\Psi_{\lambda}(\boldsymbol{r})$ vanishes at the walls and satisfies a general homogeneous boundary condition at the dot-lead interfaces: $\partial \Psi_{\lambda} / \partial n-h_{l, r} \Psi_{\lambda}=0(\hat{n}= \pm \hat{x}$ is the normal to each interface and $h_{l, r}$ are constants). A scattering solution $\Phi(\boldsymbol{r})$ at energy $E$ can be expanded $\Phi(\boldsymbol{r})=\sum_{\lambda} a_{\lambda} \Psi_{\lambda}(\boldsymbol{r})$. Since $\Phi$ and $\Psi_{\lambda}$ are solutions of the Schrödinger equation inside the dot at energies $E$ and $E_{\lambda}$, respectively, we find

$$
\begin{aligned}
a_{\lambda} & =\int_{\mathcal{A}} d A \Psi_{\lambda}^{*} \Phi \\
& =\frac{\hbar^{2}}{2 m} \frac{1}{E_{\lambda}-E} \int_{\mathcal{C}} d l\left(\Psi_{\lambda}^{*} \frac{\partial \Phi}{\partial n}-\Phi \frac{\partial \Psi_{\lambda}^{*}}{\partial n}\right) .
\end{aligned}
$$

We denote by $\phi_{c}^{i}$ a complete set of transverse wave functions, where $c$ is a channel index and $i=l, r$. For an open channel, $\phi_{c}^{i}(y)=\sqrt{2 / \mathcal{W}_{i}} \sin \left(\kappa_{c}^{i} y\right)$, where $\mathcal{W}_{i}$ is the width of the lead $i$ and $\kappa_{c}^{i}=c \pi / \mathcal{W}_{i} \quad\left(c=1,2, \ldots, \Lambda_{i}\right)$ are the quantized transverse momenta. Inside each lead we can expand $\Phi(\boldsymbol{r})=\sum_{c=1}^{\Lambda_{i}} u_{c}^{i}(x) \phi_{c}^{i}(y)$. Using Eq. (13) and the boundary conditions satisfied by the dot's eigenfunctions, the longitudinal components $u_{c}^{i}(x)$ of the scattering solution at the contact points $x=x_{l, r}$ are given by $u_{c}^{i}\left(x_{i}\right)=\sum_{i^{\prime} c^{\prime}} R_{i c ; i^{\prime} c^{\prime}}\left[\partial u_{c^{\prime}}^{i^{\prime}} / \partial n-h_{i^{\prime}} u_{c^{\prime}}^{i^{\prime}}\right]_{x=x_{i^{\prime}}}$, where

$$
R_{i^{\prime} c^{\prime} ; i c}(E)=\sum_{\lambda} \frac{y_{c^{\prime} \lambda}^{i^{\prime}} y_{c \lambda}^{i *}}{E_{\lambda}-E}
$$

is the $R$ matrix defined in terms of $y_{c \lambda}^{i}=$ $\sqrt{\hbar^{2} / 2 m} \int_{\mathcal{C}} d l \phi_{c}^{i *} \Psi_{\lambda}$, the reduced partial-level width amplitude for the decay from level $\lambda$ into channel $c$ in lead $i$. In the following, we shall omit the lead label $i$ and assume it is included in the channel label $c$.

The $S$ matrix can be expressed in terms of the $R$ matrix. To this end, it is convenient to define the $K$ matrix by $K=(k P)^{1 / 2} R(k P)^{1 / 2}$. Here $k P$ is a diagonal matrix where $k_{c}$ is the longitudinal channel momentum $\left(\hbar^{2} k_{c}^{2} / 2 m+\hbar^{2} \kappa_{c}^{2} / 2 m=E\right)$, and $P_{c}$ is the penetration factor to tunnel through the barrier in channel $c$ $\left[P_{c}=\left.k_{c}^{-1} \operatorname{Im}\left(\partial \ln u_{c}^{+} / \partial n\right)\right|_{x=x_{i}}\right.$, where $u_{c}^{+}$is the outgoing wave component of $u_{c} ; P_{c}=1$ in the absence of a barrier and $P_{c} \ll 1$ in the presence of a barrier]. In terms of the $K$ matrix (see, e.g., Blatt and Weisskopf, 1952)

$$
S=\frac{1+i K}{1-i K}
$$

Equation (14) can be rewritten for the $K$ matrix

$$
K_{c^{\prime} ; c}(E)=\frac{1}{2} \sum_{\lambda} \frac{\gamma_{c^{\prime} \lambda} \gamma_{c \lambda}^{*}}{E_{\lambda}-E}
$$

where

$$
\gamma_{c \lambda}=\sqrt{\frac{\hbar^{2} k_{c} P_{c}}{m}} \int_{\mathcal{C}} d l \phi_{c}^{*} \Psi_{\lambda}
$$

are known as the partial-width amplitudes.

The relation (16) for the $K$ matrix can also be written in an arbitrary fixed basis of wave functions (not eigenfunctions) $\rho_{j}$ in the dot:

$$
K=\pi W^{\dagger} \frac{1}{H-E} W .
$$

Here $W$ is a $N \times \Lambda$ matrix of coupling constants between the leads and the dot:

$$
W_{j c} \equiv\left(\frac{\hbar^{2} k_{c} P_{c}}{2 \pi m}\right)^{1 / 2} \int_{\mathcal{C}} d l \rho_{j}^{*} \phi_{c} .
$$

Equations (15) and (16) for the $S$ matrix can be rewritten in terms of the Green's function of an effective Hamiltonian:

$$
S=1-2 \pi i W^{\dagger} \frac{1}{E-\mathcal{H}_{\mathrm{eff}}} W,
$$

where $\mathcal{H}_{\mathrm{eff}}=H-i \pi W W^{\dagger}$. $\mathcal{H}_{\mathrm{eff}}$ is non-Hermitean; its real part is the dot's Hamiltonian $H$ and its imaginary part is the "width" matrix $\Gamma \equiv \pi W W^{\dagger}$. It generally describes the Hamiltonian of an open system that is coupled to an external region. The eigenvalues of $\mathcal{H}_{\text {eff }}$ are complex $\left(\mathcal{E}_{\lambda}-i \Gamma_{\lambda} / 2\right)$ and describe resonances of energies $\mathcal{E}_{\lambda}$ and widths $\Gamma_{\lambda}$.

\section{Resonant tunneling}

In the weak-coupling limit a typical resonance width in the dot is much smaller than the average spacing $\Delta$ between resonances. In this limit only the resonance $\lambda$ that is closest to the scattering energy $E$ contributes to the $K$ matrix (16). This leads (for $c \neq c^{\prime}$ ) to a BreitWigner resonance formula

$$
\left|S_{c^{\prime} c}\right|^{2}=\frac{\Gamma_{c^{\prime} \lambda} \Gamma_{c \lambda}}{\left(E-E_{\lambda}\right)^{2}+\left(\frac{\Gamma_{\lambda}}{2}\right)^{2}},
$$

where $\Gamma_{c \lambda} \equiv\left|\gamma_{c \lambda}\right|^{2}$ is the partial width of the resonance level $\lambda$ to decay into channel $c$, and $\Gamma_{\lambda} \equiv \sum_{c} \Gamma_{c \lambda}$ is the total width of the level. Equation (21) can also be obtained directly from Eq. (20). In the weak-coupling limit the width matrix $W W^{\dagger}$ is small (compared with $\Delta$ ), and we can diagonalize $\mathcal{H}_{\text {eff }}$ in perturbation theory. To leading order, the eigenfunctions are the same as those of $H$, and their widths (defined in terms of the eigenvalues of $\mathcal{H}_{\text {eff }}$ ) are $\Gamma_{\lambda}=2 \pi\left\langle\lambda\left|W W^{\dagger}\right| \lambda\right\rangle=\sum_{c}\left|\gamma_{c \lambda}\right|^{2}$ (where we have used $\left.\gamma_{c \lambda}=\sqrt{2 \pi} \sum_{j} W_{j c}^{*}\langle j \mid \lambda\rangle\right)$. 
The zero-temperature conductance in the tunneling regime is obtained by substituting the Breit-Wigner form [Eq. (21)] in Landauer's formula:

$$
G(E)=\frac{e^{2}}{h} \frac{\Gamma_{\lambda}^{l} \Gamma_{\lambda}^{r}}{\left(E-E_{\lambda}\right)^{2}+\left(\frac{\Gamma_{\lambda}}{2}\right)^{2}},
$$

where $\Gamma_{\lambda}^{l(r)}=\sum_{c \in l(r)} \Gamma_{c \lambda}$ is the width of the level $\lambda$ to decay into the left (right) lead and we have ignored spin degeneracy.

At finite temperature the conductance is calculated by convoluting Eq. (22) with the derivative $f^{\prime}$ of the FermiDirac distribution according to Eq. (11). In typical experiments with closed dots, $\bar{\Gamma} \ll T \ll \Delta$, and the thermal smearing factor $f^{\prime}$ does not change much over the resonance width. $G$ is then proportional to the integral of the Breit-Wigner form [Eq. (22)],

$$
G\left(E_{F}, T\right) \approx G_{\lambda}^{\text {peak }} \frac{1}{\cosh ^{2}\left(\frac{E_{\lambda}-E_{F}}{2 k T}\right)} .
$$

Here, $E_{F}$ is the Fermi energy in the leads and

$$
\begin{array}{r}
G_{\lambda}^{\text {peak }}=\frac{e^{2}}{h} \frac{\pi \bar{\Gamma}}{4 k T} g_{\lambda} \\
\text { where } g_{\lambda}=\frac{2}{\bar{\Gamma}} \frac{\Gamma_{\lambda}^{l} \Gamma_{\lambda}^{r}}{\Gamma_{\lambda}^{l}+\Gamma_{\lambda}^{r}} .
\end{array}
$$

Equation (23) describes a conductance peak of width $\sim$ $k T$ that is centered at $E_{F}=E_{\lambda}$ and has a peak-height amplitude of $G_{\lambda}^{\text {peak }}$.

\section{E. Coulomb blockade}

Coulomb blockade occurs when an "island" of electrons is weakly coupled to two leads via tunnel junctions. When the coupling is weak, the conductance drops below $e^{2} / h$ and the charge on the dot becomes quantized. The linear conductance of the dot oscillates as a function of the gate voltage with a period that corresponds to the addition of a single electron to the dot. For reviews on Coulomb blockade, see Averin and Likharev (1991) and van Houten, Beenakker, and Staring (1992). For recent experimentally oriented reviews on transport in Coulomb-blockade quantum dots, see Meirav and Foxman (1995), Kouwenhoven, Marcus et al. (1997), and Kouwenhoven and McEuen (1998).

Coulomb-blockade oscillations were first observed in a metallic grain (Giaever and Zeller, 1968), where $\Delta \ll k T$ and the spectrum could be treated as a quasicontinuum. A transport theory for this classical regime was developed by Shekhter (1972) and Kulik and Shekhter (1975). The total classical electrostatic energy of a Coulomb island with $\mathcal{N}$ electrons is

$$
U(\mathcal{N})=-Q V_{\text {ext }}+Q^{2} / 2 C=-\mathcal{N} e V_{\text {ext }}+\mathcal{N}^{2} e^{2} / 2 C
$$

where $V_{\text {ext }}$ is the potential difference between the electron gas and the reservoir (due to a gate voltage), and $C$ is the total capacitance between the island and its surroundings. By defining an externally induced charge variable $Q_{\text {ext }} \equiv C V_{\text {ext }}$, we can write Eq. $(25)$ as $U(\mathcal{N})=$ $\left(Q-Q_{\text {ext }}\right)^{2} / 2 C$ up to an additive constant. $Q_{\text {ext }}$ can be varied continuously by changing $V_{\text {ext }}$. The number of electrons in the dot for a given $V_{\text {ext }}$ is determined by minimizing $U(\mathcal{N})$. When $Q_{\text {ext }}=\mathcal{N} e$, the minimum is obtained for the state with charge $Q=\mathcal{N} e$, and the energy of the states with $Q=(\mathcal{N} \pm 1) e$ is higher by $e^{2} / 2 C$. As a result, the tunneling density of states has a gap of $E_{C}=e^{2} / C$ around the Fermi energy, blocking the flow of electrons into the island. This situation is demonstrated in panels (a) and (c) of Fig. 8. However, when $Q_{\text {ext }}=(\mathcal{N}+1 / 2) e$, both states $Q=\mathcal{N} e$ and $Q=(\mathcal{N}+1) e$ are degenerate (Glazman and Shekhter, 1989), allowing the tunneling of one more charge into the metallic particle [see panels (b) and (d) of Fig. 8]. The conductance is maximal at this degeneracy point.

In semiconductor quantum dots the mean level spacing is much larger than in metal grains of a similar size, and experiments can easily probe the quantum Coulombblockade regime $T<\Delta \ll e^{2} / C$. A simple Hamiltonian for the dot can be written by assuming electrons in a one-body confining potential plus an electrostatic energy [Eq. (25)]:

$$
H_{\mathrm{dot}}=\sum_{\lambda}\left(E_{\lambda}-e \alpha V_{g}\right) a_{\lambda}^{\dagger} a_{\lambda}+e^{2} \hat{\mathcal{N}}^{2} / 2 C,
$$

where $a_{\lambda}^{\dagger}|0\rangle$ is a complete set of single-particle eigenstates in the dot with energies $E_{\lambda}$, and $\hat{\mathcal{N}}=\sum_{\lambda} a_{\lambda}^{\dagger} a_{\lambda}$ is the electron number operator in the dot. The external potential of Eq. (25) $V_{\text {ext }}=\alpha V_{g}$ is written in terms of a gate voltage $V_{g}$ and $\alpha=C_{g} /\left(C_{g}+C_{\text {dot }}\right)$, where $C_{g}$ is the gate-dot capacitance and $C_{\text {dot }}$ is the dot-leads capacitance. The Hamiltonian (26) is known as the constantinteraction model, since only the average constant part of the electron-electron interaction $\left(\hat{\mathcal{N}}^{2} e^{2} / 2 C\right)$ is taken into account.

When $T \ll \Delta$, conductance is possible only by resonant tunneling through a corresponding quantized level in the dot. Resonant tunneling of the $\mathcal{N}$ th electron occurs when the total energy before and after the tunneling event is conserved: $E_{F}+U(\mathcal{N}-1)=E_{\mathcal{N}}+U(\mathcal{N})$. Using Eq. (25) we find that the effective Fermi energy $\tilde{E}_{F} \equiv E_{F}+e \alpha V_{g}$ satisfies $\tilde{E}_{F}=E_{\mathcal{N}}+\left(\mathcal{N}-\frac{1}{2}\right)\left(e^{2} / C\right)$. The spacing between Coulomb-blockade peaks is now given by

$$
\Delta_{2} \equiv \Delta \tilde{E}_{F}=\left(E_{\mathcal{N}+1}-E_{\mathcal{N}}\right)+e^{2} / C
$$


The charging energy is usually much larger than the mean level spacing in the dot so that the Coulombblockade peaks are almost equidistant. Figure 8 illustrates the phenomenon of Coulomb blockade in the quantum regime.
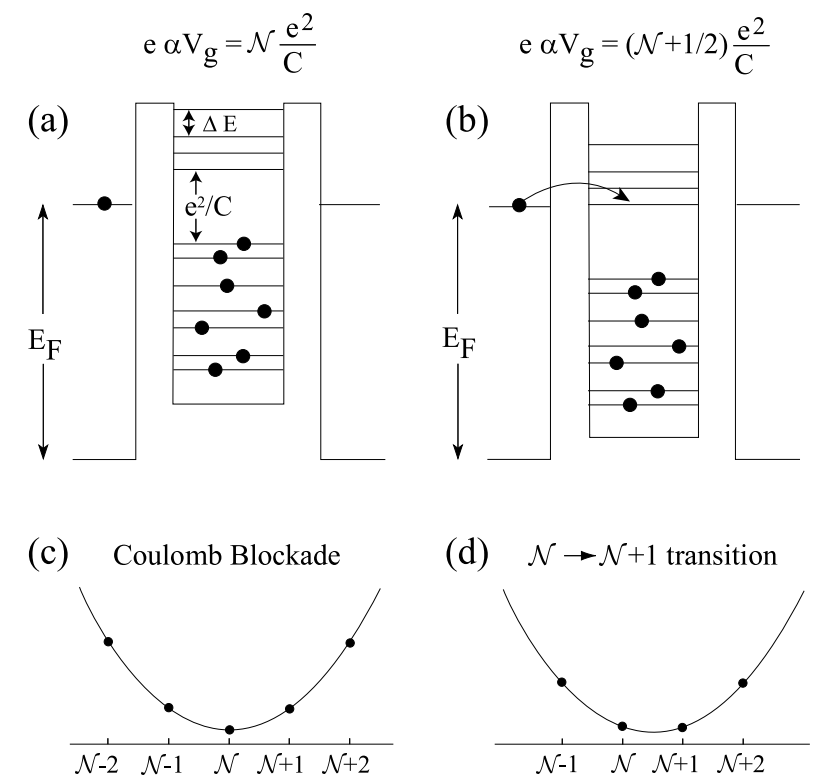

FIG. 8. Schematic illustration of Coulomb blockade in an almost-closed dot. When the gate voltage is tuned to a value $e \alpha V_{g}=\mathcal{N} e^{2} / C$ [panel (a)] there is a charging energy gap in the single-particle spectrum on both sides of the Fermi energy, blocking the tunneling of electrons into the dot. On the other hand, when the gate voltage increases to $e \alpha V_{g}=(\mathcal{N}+1 / 2) e^{2} / C$ [panel (b)], it compensates for the Coulomb repulsion, and the charging energy gap for adding an electron to the dot vanishes. When the Fermi energy in the leads matches the first unoccupied single-particle state in the dot, resonant tunneling of an electron into the dot occurs. Panels (c) and (d) show the total electrostatic energy $U(\mathcal{N})$ of the dot vs the number of electrons [see Eq. (25)]: (c) for $e \alpha V_{g}=\mathcal{N} e^{2} / C$, the energy of the dot is minimal for $\mathcal{N}$ electrons in the dot, leading to charge quantization; (d) when $e \alpha V_{g}=(\mathcal{N}+1 / 2) e^{2} / C$, the energies of a dot with $\mathcal{N}$ and $\mathcal{N}+1$ electrons are equal and the dot's charge can fluctuate between $\mathcal{N} e$ and $(\mathcal{N}+1) e$. This is known as the degeneracy point.

At $T \sim \Delta$, several resonances contribute to the conductance peak. A finite-temperature theory for the onresonance conductance was derived by Beenakker (1991) and Meir, Wingreen, and Lee (1991) using linear response theory, and by Averin, Korotkov, and Likharev (1991) in the nonlinear $I-V$ regime.

For $k T \gg \Gamma$ (where $\Gamma$ is a typical level width), the coherence between the electrons in the leads and in the dot can be ignored, and a master-equations approach is valid. Beenakker (1991) used the constantinteraction model (26), where an eigenstate of the dot is described by a set of occupation numbers $\boldsymbol{n} \equiv\left\{n_{\lambda}\right\}$ of the single-particle levels, and its energy is $\mathcal{E}(\boldsymbol{n})=$ $\sum_{\lambda} E_{\lambda} n_{\lambda}+U(\mathcal{N})$. The dot is connected to left and right reservoirs that are in thermal equilibrium at temperature $T$ and Fermi energy $E_{F}$. At equilibrium $P_{e q}(\boldsymbol{n})=$ $\mathcal{Z}^{-1} \exp \left\{-\left[\mathcal{E}(\boldsymbol{n})-\mathcal{N} E_{F}\right] / k T\right\}$, where $\mathcal{Z}$ is the grandcanonical partition function. A small source-drain voltage $V_{s d}$ is applied between the left and right reservoirs, causing a current $I$ through the dot (see Fig. 2), and the linear conductance is calculated from $G=I / V_{s d}$. The voltage drop is $\eta V_{s d}$ across the left barrier, and $(1-\eta) V_{s d}$ across the right barrier. The current through the left lead is described by the net flux of electrons that tunnel in or out of the dot across the left barrier

$$
\begin{gathered}
I=-e \sum_{\lambda} \sum_{\boldsymbol{n}} P(\boldsymbol{n}) \frac{\Gamma_{\lambda}^{l}}{\hbar}\left\{\delta_{n_{\lambda}, 0} f\left(\tilde{E}_{\lambda}(\mathcal{N}+1)+\eta e V_{s d}\right)\right. \\
\left.-\delta_{n_{\lambda}, 1}\left[1-f\left(\tilde{E}_{\lambda}(\mathcal{N})+\eta e V_{s d}\right)\right]\right\},
\end{gathered}
$$

where $f(x)=[1+\exp (x / k T)]^{-1}$ is the Fermi-Dirac distribution in the reservoirs, $\tilde{E}_{\lambda}(\mathcal{N}) \equiv E_{\lambda}-\left(\tilde{E}_{F}-(\mathcal{N}-\right.$ $\left.1 / 2) e^{2} / C\right)$, and $\Gamma_{\lambda}^{l} / \hbar$ is the tunnel rate from level $\lambda$ to the left lead. The first term on the right-hand side of Eq. (28) describes the tunneling of electrons from a level in the left lead with filling $f$ into an empty level $\lambda$ in the dot, while the second term represents the tunneling of an electron from an occupied state $\lambda$ in the dot to a level in the left lead with emptiness of $1-f$. Only energyconserving transitions are taken into account, and the summation over $\lambda$ accounts for the possibility of tunneling through different levels $\lambda$.

The nonequilibrium distribution $P(\boldsymbol{n})$ of electrons in the dot satisfies a set of master equations

$$
\frac{\partial P(\boldsymbol{n})}{\partial t}=\sum_{\boldsymbol{m}}\left[P(\boldsymbol{m}) \mathcal{W}_{\boldsymbol{m} \rightarrow \boldsymbol{n}}-P(\boldsymbol{n}) \mathcal{W}_{\boldsymbol{n} \rightarrow \boldsymbol{m}}\right]
$$

where $\mathcal{W}_{\boldsymbol{m} \rightarrow \boldsymbol{n}}$ is the transition rate from state $\boldsymbol{m}$ to state $\boldsymbol{n}$. Only states $\boldsymbol{m}$ that differ by one electron from the state $\boldsymbol{n}$ appear in the sum in Eq. (29), and the respective transition rates are

$$
\begin{aligned}
\mathcal{W}_{1_{\lambda} \rightarrow 0_{\lambda}}= & \frac{\Gamma_{\lambda}^{l}}{\hbar}\left[1-f\left(\tilde{E}_{\lambda}(\mathcal{N}+1)+\eta e V_{s d}\right)\right] \\
& +\frac{\Gamma_{\lambda}^{r}}{\hbar}\left[1-f\left(\tilde{E}_{\lambda}(\mathcal{N}+1)-(1-\eta) e V_{s d}\right)\right] \\
\mathcal{W}_{0_{\lambda} \rightarrow 1_{\lambda}}= & \frac{\Gamma_{\lambda}^{l}}{\hbar} f\left(\tilde{E}_{\lambda}(\mathcal{N})+\eta e V_{s d}\right) \\
& +\frac{\Gamma_{\lambda}^{r}}{\hbar} f\left(\tilde{E}_{\lambda}(\mathcal{N})-(1-\eta) e V_{s d}\right)
\end{aligned}
$$

where $\mathcal{N}=\sum_{\mu} n_{\mu}$ is the number of electrons in the final state $\boldsymbol{n}$. In the notation used in Eq. (30), $1_{\lambda} \rightarrow 0_{\lambda}$, for example, denotes the transition $\boldsymbol{m} \rightarrow \boldsymbol{n}$ with $m_{\lambda}=$ $1, n_{\lambda}=0$, and $m_{\mu}=n_{\mu}$ for all $\mu \neq \lambda$. The term $\mathcal{W}_{1_{\lambda} \rightarrow 0_{\lambda}}$ describes the tunneling of an electron from a filled $\lambda$ level in a dot with $\mathcal{N}+1$ electrons into a level in the left and right leads with emptiness of $1-f$, while $\mathcal{W}_{0_{\lambda} \rightarrow 1_{\lambda}}$ 
corresponds to the tunneling of an electron from a level in the left or right lead with filling $f$ into a $\operatorname{dot}$ with $\mathcal{N}-1$ electrons.

We are interested only in the stationary state $\partial P(\boldsymbol{n}) / \partial t=0$, leading to detailed balance equations $P\left(1_{\lambda}\right) \mathcal{W}_{1_{\lambda} \rightarrow 0_{\lambda}}=P\left(0_{\lambda}\right) \mathcal{W}_{0_{\lambda} \rightarrow 1_{\lambda}}$. A solution to the detailed balance equations in the linear response regime (Beenakker, 1991) is of the form $P(\boldsymbol{n}) \approx P_{e q}(\boldsymbol{n})\left[1+\left(e V_{s d} / k T\right) L(\boldsymbol{n})\right]$, where $L(\boldsymbol{n})=$ $\sum_{\lambda}\left[\Gamma_{\lambda}^{r} /\left(\Gamma_{\lambda}^{l}+\Gamma_{\lambda}^{r}\right)-\eta\right] n_{\lambda}+$ const. Calculating the current (28) to first order in $V_{s d}$ using the solution for $P(\boldsymbol{n})$, one obtains the conductance $G\left(T, \tilde{E}_{F}\right)$ as a function of the temperature and the effective Fermi energy

$$
G\left(T, \tilde{E}_{F}\right)=\frac{e^{2}}{h} \frac{\pi \bar{\Gamma}}{4 k T} g \text {, where } g=\sum_{\lambda} w_{\lambda}\left(T, \tilde{E}_{F}\right) g_{\lambda}
$$

is the dimensionless conductance expressed as a thermal average over the level conductances $g_{\lambda}=$ $2 \bar{\Gamma}^{-1} \Gamma_{\lambda}^{l} \Gamma_{\lambda}^{r} /\left(\Gamma_{\lambda}^{l}+\Gamma_{\lambda}^{r}\right)$. The thermal weights are given by

$$
\begin{array}{rl}
w_{\lambda}=\sum_{\mathcal{N}} & 4 P_{\mathcal{N}}\left\langle n_{\lambda}\right\rangle_{\mathcal{N}} \\
\times & {\left[1-f\left(E_{\lambda}+(\mathcal{N}-1 / 2) \frac{e^{2}}{C}-\tilde{E}_{F}\right)\right],}
\end{array}
$$

where $P_{\mathcal{N}}$ is the probability that the $\operatorname{dot}$ has $\mathcal{N}$ electrons, and $\left\langle n_{\lambda}\right\rangle_{\mathcal{N}}$ is the canonical occupation of a level $\lambda$. The contribution to $w_{\lambda}$ in Eq. (32) from a fixed number of electrons $\mathcal{N}$ corresponds to the product of the probability that level $\lambda$ is occupied in a dot with $\mathcal{N}$ electrons and the probability that a state in the lead is empty at the same total energy. The probability $P_{\mathcal{N}}$ is given by $P_{\mathcal{N}}=\exp [-\Omega(\mathcal{N}) / k T] / \sum_{\mathcal{N}^{\prime}} \exp \left[-\Omega\left(\mathcal{N}^{\prime}\right) / k T\right]$, where $\Omega(\mathcal{N}) \equiv F(\mathcal{N})+U(\mathcal{N})-\mathcal{N} E_{F}$ and $F(\mathcal{N})$ is the canonical noninteracting free energy.

In typical experiments, $T, \Delta \ll e^{2} / C$, and only one term in the sum of Eq. (32) contributes to a given conductance peak. For gate voltages that are tuned to a conductance peak between $\mathcal{N}-1$ and $\mathcal{N}$ electrons in the dot, we have

$$
w_{\lambda}=4 f\left(\Delta F_{\mathcal{N}}-\tilde{E}_{F}\right)\left\langle n_{\lambda}\right\rangle_{\mathcal{N}}\left[1-f\left(E_{\lambda}-\tilde{E}_{F}\right)\right],
$$

where $\Delta F_{\mathcal{N}} \equiv F(\mathcal{N})-F(\mathcal{N}-1)$, and we have used $P_{\mathcal{N}} \approx f(\Omega(\mathcal{N})-\Omega(\mathcal{N}-1))$. In Eq. (33) and in the following, $\tilde{E}_{F}$ (or equivalently $e \alpha V_{g}$ ) is measured relative to $(\mathcal{N}-1 / 2) e^{2} / C$.

In the limit $k T \ll \Delta$ and spin-nondegenerate levels, only one level $\lambda=\mathcal{N}$ contributes to Eq. (31). Furthermore $\Delta F_{\mathcal{N}} \approx E_{\mathcal{N}}$, and using $f(x)[1-f(x)]=$ $-k T f^{\prime}(x)$, Eq. $\quad(33)$ becomes $w_{\mathcal{N}}(k T \ll \Delta)=$ $\cosh ^{-2}\left[\left(E_{\mathcal{N}}-\tilde{E}_{F}\right) / 2 k T\right]$, so that Eq. (31) reduces to Eqs. (23) and (24). However, if the electron tunnels into an empty level $\lambda$ that is spin degenerate (with spin $1 / 2)$, we find a conductance peak $G\left(\tilde{E}_{F}, T\right)=$ $\left(e^{2} / h\right)(2 \pi \bar{\Gamma} / k T)(2 / \bar{\Gamma})\left[\Gamma_{\lambda}^{l} \Gamma_{\lambda}^{r} /\left(\Gamma_{\lambda}^{l}+\Gamma_{\lambda}^{r}\right)\right]\left[1-f\left(E_{\lambda}-\tilde{E}_{F}\right)\right]$ $\times\left[2+e^{\left(E_{\lambda}-\tilde{E}_{F}\right) / k T}\right]^{-1}$ (Glazman and Matveev, 1988). The conductance maximum is shifted to $\tilde{E}_{F}=E_{\lambda}-$ $(k T \ln 2) / 2$, and the peak height in Eq. (24) is rescaled by a factor of $8(\sqrt{2}-1)^{2} \approx 1.37$. In this case, the scattering approach of Sec. II.D leads to a conductance peak as in (23), with a peak height (24) scaled by a factor of 2 . This result is wrong since it ignores charging energy.

Similarly, if the method of Sec. II.D (valid in the absence of charging energy) is applied at temperatures $T \sim \Delta$, we obtain Eq. (31), but with weights

$$
w_{\lambda}^{(0)}=-4 k T f^{\prime}\left(E_{\lambda}-\tilde{E}_{F}\right) .
$$

Indeed in the limit $e^{2} / C \rightarrow 0$, all terms in Eq. (32) contribute. The factor $1-f$ becomes independent of $\mathcal{N}$, $\sum_{\mathcal{N}} P_{\mathcal{N}}\left\langle n_{\lambda}\right\rangle_{\mathcal{N}} \equiv f\left(E_{\lambda}-\tilde{E}_{F}\right)$, and the weights $w_{\lambda}$ reduce to Eq. (34). However, for $e^{2} / C \gg \Delta$, only $P_{\mathcal{N}}$ and $P_{\mathcal{N}-1}$ are non-negligible, as states with number of electrons different from $\mathcal{N}$ and $\mathcal{N}-1$ are pushed away by the charging energy. We conclude that, in the presence of charging energy, the approach based on the Breit-Wigner and Landauer formulas breaks down at finite temperature and Eq. (34) must be replaced by Eq. (33).

The discreteness of the spectrum is unimportant at high temperatures $k T \gg \Delta$ where the canonical occupations can be approximated by the Fermi-Dirac distribution. Assuming that the tunneling rates $\Gamma^{l(r)} / \hbar$ depend only weakly on energy, one finds (for $\Delta \ll k T \ll e^{2} / C$ )

$$
\begin{aligned}
& G\left(\tilde{E}_{F}, T\right) \approx G_{\lambda}^{\text {peak }} \frac{1}{\cosh ^{2}\left(\frac{\mu-\tilde{E}_{F}}{2.5 k T}\right)} ; \\
& G_{\lambda}^{\text {peak }}=\frac{e^{2}}{h} \frac{\pi}{\Delta} \frac{\Gamma^{l} \Gamma^{r}}{\Gamma^{l}+\Gamma^{r}},
\end{aligned}
$$

where $\Gamma^{l(r)}$ are the energy-averaged partial widths. Comparing with the corresponding formulas in the quantum limit $k T \ll \Delta$ [Eqs. (23) and (24)], we see that for the same temperature, the line shape is similar but with a somewhat larger width in the classical limit. The peak height in the classical regime is temperature independent since $\sim k T / \Delta$ levels contribute, canceling the $1 / T$ dependence of the peak height in the quantum regime. Furthermore, the widths $\Gamma^{l(r)}$ in the classical formula (35) are energy averaged, and thus lead to smooth variation of the peak heights as a function of $V_{g}$. In contrast, the peak heights exhibit strong fluctuations in the quantum regime [see, for example, the peak series in Fig. 7(c)].

If $k T \gg e^{2} / C$, the charging energy is not important and Landauer's formula reproduces the correct conductance $G^{(0)}=2\left(e^{2} / h\right)(\pi / \Delta) \Gamma^{l} \Gamma^{r} /\left(\Gamma^{l}+\Gamma^{r}\right)$. Coulombblockade oscillations disappear and the conductance is large as its peak height in the classical Coulomb-blockade regime. 


\section{F. Cotunneling}

The off-resonance conductance can be calculated using perturbation theory (in the dot-leads coupling). Firstorder processes are forbidden by energy conservation because of the charging energy gap, so the leading-order contribution is second order. The main second-order tunneling mechanism is known as cotunneling (Averin and Nazarov, 1990; Glazman and Matveev, 1990a). In inelastic cotunneling, an electron that tunnels from the left lead into a state in the dot is followed by an electron that tunnels from a different state of the dot into the right lead. In elastic cotunneling, an electron tunnels into the dot (from the left lead) and out of the dot (into the right lead) through the same intermediate state of the dot. Also contributing to cotunneling are holes that move from the right to the left lead, describing first an electron that tunnels out of the dot into the right lead, followed by an electron that tunnels from the left lead into the dot. The transitions involved in cotunneling are nonenergy-conserving and therefore virtual. The intermediate single-particle states in the dot are separated from the Fermi energy in the leads by a gap $E_{e}$ for the virtual tunneling of an electron to a state above the Fermi energy, and by a gap $E_{h}$ for the virtual tunneling of a hole to a state below the Fermi energy $\left(E_{e}+E_{h}=E_{C}=e^{2} / C\right)$.

We restrict the discussion to low temperatures $k T<$ $\sqrt{E_{C} \Delta}$, where elastic cotunneling dominates (Averin and Nazarov, 1990). The dot-leads Hamiltonian is described by

$$
\begin{aligned}
H= & +\sum_{k, c \in l, r} E_{k c} c_{k c}^{\dagger} c_{k c} \\
& +\sum_{\substack{k, c \in l, r \\
\lambda}}\left(V_{k c, \lambda} c_{k c}^{\dagger} a_{\lambda}+H . c .\right)
\end{aligned}
$$

where $H_{\text {dot }}$ is the dot's Hamiltonian (26), $c_{k c}^{\dagger}$ creates an electron with wave number $k$ in channel $c$ in either the left $(l)$ or right $(r)$ lead with energy $E_{k c}$, and $V$ is a tunneling matrix element between the left or right lead and the dot. The elastic cotunneling conductance is

$$
G=\frac{e^{2}}{h} \sum_{c \in l, c^{\prime} \in r}\left|\mathcal{T}_{c^{\prime} c}\right|^{2}
$$

where

$$
\begin{aligned}
\mathcal{T}_{c^{\prime} c}=-\sum_{E_{\lambda}>E_{F}} & \frac{\gamma_{c^{\prime} \lambda}^{r *} \gamma_{c \lambda}^{l}}{\left|E_{\lambda}-E_{F}\right|+E_{e}} \\
& +\sum_{E_{\lambda} \leq E_{F}} \frac{\gamma_{c^{\prime} \lambda}^{r *} \gamma_{c \lambda}^{l}}{\left|E_{F}-E_{\lambda}\right|+E_{h}}
\end{aligned}
$$

is the elastic cotunneling amplitude from channel $c$ in the left lead to channel $c^{\prime}$ in the right lead. We have defined $\gamma_{c \lambda}^{l(r)}=\sqrt{2 \pi \rho_{c}} V_{c \lambda}^{l(r)}\left[\rho_{c}(E)=\sum_{k} \delta\left(E-\epsilon_{k c}\right)\right.$ is the lead density of states in channel $c$ ] to be the partial-width amplitude of an electron in a state $\lambda$ to decay into channel $c$ in the left (right) lead. The cotunneling amplitude contains contributions from both particle $\left(E_{\lambda}>E_{F}\right)$ and hole $\left(E_{\lambda} \leq E_{F}\right)$ states. Each term in the sum over particle (hole) states is the amplitude for an electron (hole) to tunnel from the left (right) lead to the right (left) lead through an intermediate state $\lambda$. Expression (38) assumes that both $E_{e}$ and $E_{h}$ are of the order of $E_{C}$, and thus $\gg \Delta$. In contrast to the conductance at the peak, a large number of excited states in the dot contribute to the off-resonance conductance.

\section{G. Nonlinear transport}

Thus far we have considered only the linear conductance. This corresponds to a source-drain voltage $e V_{s d}$ that is smaller than a typical level spacing $\Delta$ in the dot. The electron can then tunnel only through the lowest unoccupied level, and the observed Coulomb-blockade oscillations provide information on the ground states of the dot with increasing number of electrons. More generally, the current depends on the number of available states in the dot between the chemical potentials in the left and right leads. Since the difference between the chemical potentials is $e V_{s d}$, we expect that as $V_{s d}$ increases additional states in the dot become available for tunneling. A nonlinear transport theory in quantum dots was developed by Averin and Korotkov (1990) and Averin, Korotkov, and Likharev (1991). In the classical regime, the current increases in steps as a function of $V_{s d}$ (Coulomb staircase), corresponding to the increase in the number of available charge states in the dot. In the quantum regime, the current depends on the number of available excited levels in the dot through which a fixed number of electrons can tunnel (Johnson et al., 1992). Thus nonlineartransport measurements in the quantum regime provide information on the excitation spectrum of a dot with a fixed number of electrons.

In practice, the current through the dot can be measured as a function of both $V_{s d}$ and a gate voltage $V_{g}$. For a fixed $e V_{s d}$ below the first excited state in the dot, the current versus $V_{g}$ displays the usual Coulomb-blockade peaks of the linear regime. However, when $e V_{s d}$ is above the first excited state in the dot, the electron can tunnel through two states in the dot, and each single peak develops into a double peak. Similarly, as $e V_{s d}$ increases above the second excited state, each Coulomb-blockade oscillation is composed of three peaks. The differential conductance $d I / d V_{s d}$ displays a peak when a level in the dot matches the chemical potential in one of the leads. From the spacings among the peaks in each oscillation, it is possible to infer the excitation spectrum of the dot. The differential conductance forms a diamond-shaped diagram 
in the $V_{s d^{-}} V_{g}$ plane (McEuen et al., 1993). Each diamond corresponds to a Coulomb-blockaded region with a certain number of electrons on the dot.

\section{STATISTICAL THEORY: FROM DISORDERED METALS TO BALLISTIC DOTS}

The phase coherence of transport in mesoscopic structures leads to quantum interference effects. Consequently, the conductance in these structures exhibits fluctuations as a function of experimentally controllable parameters, such as magnetic field, gate voltage, or sample. Such fluctuations were first observed in disordered metals. To describe the statistical properties of these fluctuations, we assume that the impurity configurations in the sample constitute an ensemble. Similar samples fabricated by similar methods differ from each other in the details of their impurity configurations, and each can be thought of as a different member of the ensemble. For early reviews on quantum interference effects in mesoscopic structures, see Altshuler, Lee, and Webb (1991), Beenakker and van Houten (1991), and Washburn and Webb (1993). See also the books by Datta (1995) and Imry (1996).

In the last decade it has become possible to produce relatively clean high-mobility GaAs quantum dots. These ballistic devices also exhibit conductance fluctuations. In irregularly shaped dots, where the classical dynamics of the electron is chaotic, the fluctuations are universal and depend only on the symmetry class and the transmission properties of the leads. The physical origin of the fluctuations in ballistic dots is similar to that in diffusive structures, namely quantum interference effects. The statistical properties of these fluctuations are also assumed to be described by an appropriate ensemble. In practice, the concept of an ensemble (for both diffusive and ballistic systems) is justified by the ergodic hypothesis. These systems' statistical properties result from averaging over energy, shape, magnetic field, etc.

The diagrammatic and semiclassical methods played a key role in our theoretical understanding of disordered structures and ballistic dots, respectively. Two additional powerful approaches contributed to recent progress in the field: random-matrix theory (RMT) and the supersymmetry method. Random-matrix theory originated in nuclear physics (Wigner, 1958; see also Wigner, 1951, $1955,1957)$, and was later conjectured to describe the universal quantal fluctuations in systems whose associated classical dynamics is chaotic (Bohigas, Giannoni, and Schmit, 1984). The supersymmetry method was originally conceived as a method for carrying out exact ensemble averages in disordered systems (Efetov, 1983). In a certain regime it is equivalent to RMT, but more generally it can be used to derive nonuniversal corrections.
Section III.A introduces the relevant scales and commonly used models for disordered and ballistic quantum dots. The following sections review the principal methods of the statistical approach. Section III.B outlines the semiclassical approach. The semiclassical treatment of disordered systems has mostly reproduced results originally derived in impurity perturbation theory, but it offers a more intuitive approach. The diagrammatic approach is not discussed here; its main lines can be found in Abrikosov, Gor'kov, and Dzhyaloshinskii (1963) and Altshuler and Simons (1995). Section III.C reviews the RMT approach, and Sec. III.D gives a brief introduction to the supersymmetry method.

\section{A. Disordered metals and ballistic dots}

\section{Scales in the diffusive regime}

In a mesoscopic structure, the coherence length exceeds the system's size: $L_{\phi}>L$ (see Sec. I). Other relevant length scales in disordered systems are the mean free path $l$ and the Fermi wavelength $\lambda_{F}$. The diffusive regime corresponds to $l \ll L$. In the metallic or weakly disordered regime, the Fermi wavelength is much smaller than the elastic mean free path: $\lambda_{F} \ll l$ (i.e., $k_{F} l \gg 1$ ).

Another important length scale in disordered systems is the localization length $\xi$ over which the electron's wave function is localized (for a review of localization theory see Lee and Ramakrishnan, 1985). In the metallic regime, the localization length is large, and we shall restrict our discussions to the nonlocalized regime where $\xi \gg L$.

The time the electron takes to diffuse across the length $L$ of a disordered sample is $\tau_{D}=L^{2} / D$, where $D$ is the diffusion coefficient. The associated energy scale

$$
E_{c} \equiv \frac{\hbar}{\tau_{D}}=\frac{\hbar D}{L^{2}}
$$

is known as the Thouless energy (see Sec. I.C). The Thouless energy can be directly related to the conductance. The conductance $G$ of a homogeneous conductor in $d$ dimensions is $G \sim \sigma L^{d-2}$, where $\sigma$ is the conductivity. Using Einstein's relation (4) for $\sigma$, we can write

$$
G \sim \frac{e^{2}}{\hbar}\left(\nu L^{d}\right)\left(\frac{\hbar D}{L^{2}}\right)=\frac{e^{2}}{\hbar} \frac{E_{c}}{\Delta} .
$$

The dimensionless Thouless conductance ${ }^{4} g_{T}$ is defined by $G \equiv\left(e^{2} / \hbar\right) g_{T}$, and according to Eq. (40)

\footnotetext{
${ }^{4}$ The dimensionless Thouless conductance is usually denoted by $g$. In this review we denote it by $g_{T}$ since $g$ is used to denote the dimensionless conductance peak height in a closed dot [see, e.g., Eq. (31)] and the dimensionless conductance in an open dot (see, e.g., Sec. IV.C.1).
} 


$$
g_{T}=\frac{E_{c}}{\Delta} \equiv N\left(E_{c}\right),
$$

i.e., $g_{T}$ measures the number of levels in an energy interval $E_{c}$. In $2 \mathrm{D}$ systems, $g_{T}=l / \lambda_{F}$, and the dimensionless conductance is therefore large in the metallic regime.

The relations among relevant length scales can be translated into relations among the corresponding energy and time scales. The following relations are useful:

$$
\frac{\hbar / \tau}{E_{c}}=d\left(\frac{L}{l}\right)^{2} ; \quad \frac{E_{F}}{\hbar / \tau}=\frac{1}{2} k_{F} l
$$

where $\tau$ is the mean free time. It follows that the diffusive regime is characterized by $E_{c} \ll \hbar / \tau$, i.e., the time to diffuse across the sample is large compared with the mean free time: $\tau_{D} \gg \tau$. In a good metal $\hbar / \tau \ll E_{F}$ and $E_{c} \gg \Delta$.

In summary, the following inequalities hold in a disordered metal in its diffusive regime:

$$
\begin{gathered}
\Delta \ll E_{c} \ll \hbar / \tau \ll E_{F}, \\
\tau_{H} \gg \tau_{D} \gg \tau \gg \hbar / E_{F},
\end{gathered}
$$

where $\tau_{H}=h / \Delta$ is the Heisenberg time. The energy region above $\hbar / \tau$ corresponds to ballistic motion of the electron since the corresponding time scale is shorter than the average time between scatterings from the impurities. In the energy range between $E_{c}$ and $\hbar / \tau$, the dynamics are diffusive, but there is not sufficient time for the electron to reach the boundaries. Energy scales below $E_{c}$ correspond to time scales in which the electron has reached the system's boundaries and the diffusive motion has explored the full length of the structure. We shall see (e.g., in Sec. III.B.1) that this is the regime where the fluctuations are universal (known as the ergodic regime).

\section{Scales in the ballistic regime}

For weaker disorder and/or a smaller sample, the mean free time $\tau$ increases and/or the time $\tau_{D}$ to diffuse across the dot decreases, and eventually the Thouless energy $E_{c}$ exceeds $\hbar / \tau$. In this limit the system can be considered clean, and the dynamics across its length are ballistic. In terms of length scales, the ballistic regime is defined by $L \ll l$.

In ballistic structures $\tau_{D}$ is meaningless and another time scale becomes relevant: the ergodic time $\tau_{c}$, which is of the order of the time of flight across the sample. The ergodic time plays the same role in ballistic systems that the diffusion time $\tau_{D}$ plays in disordered systems. The quantity analogous to the Thouless energy is $E_{T} \equiv \hbar / \tau_{c}$, sometimes called the ballistic Thouless energy. The ballistic dimensionless conductance is $g_{T}=E_{T} / \Delta=\tau_{H} / 2 \pi \tau_{c}$. In $2 \mathrm{D}$ we can estimate $g_{T}$ from $\tau_{c} \sim L / v_{F}$ and $\tau_{H}=h \nu \mathcal{A}$ to be $g_{T} \sim \mathcal{N}^{1 / 2} / 2 \pi$ (where $\mathcal{N}$ is the number of electrons in the dot).

Within the ballistic regime (where $\hbar / \tau \ll \hbar / \tau_{c}$ ), it is possible to distinguish two cases depending on the relation between $\hbar / \tau$ and $\Delta$ (Altland and Gefen, 1993). When $\Delta \ll \hbar / \tau \ll \hbar / \tau_{c}$, a typical impurity matrix element can mix many levels, while for $\hbar / \tau \ll \Delta, \hbar / \tau_{c}$ the disorder is very weak and can be treated in low-order perturbation theory.

\section{Models of disordered structures and ballistic dots}

The dynamics of a single electron in d dimensions is described by Schrödinger's equation

$$
\frac{1}{2 m^{*}}\left(\boldsymbol{p}+\frac{e}{c} \boldsymbol{A}\right)^{2} \Psi+V \Psi=E \Psi
$$

where $V(\boldsymbol{r})$ is a one-body potential and $\boldsymbol{A}(\boldsymbol{r})$ is a vector potential describing a magnetic field. The disorder is modeled by an ensemble of random potentials $\{V(\boldsymbol{r})\}$. Often this ensemble is taken to be Gaussian with

$$
\overline{V(\boldsymbol{r})}=0 ; \quad \overline{V(\boldsymbol{r}) V\left(\boldsymbol{r}^{\prime}\right)}=\frac{\hbar}{2 \pi \nu \tau} \delta\left(\boldsymbol{r}-\boldsymbol{r}^{\prime}\right)
$$

The parametrization of the strength of the disorder in terms of the impurity scattering rate $1 / \tau$ is obtained in the Born limit. A discretized version of this model is known as the tight-binding Anderson model (Anderson, 1958). In second-quantized form

$$
H=-\sum_{\langle m, n\rangle}\left(t_{m n} e^{i \theta_{m n}} a_{m}^{\dagger} a_{n}+\text { h.c. }\right)+\sum_{m} V_{m} a_{m}^{\dagger} a_{m},
$$

where $a_{m}^{\dagger}$ creates an electron at site $m, t_{m n}=\hbar^{2} / 2 m^{*} a^{2}$ ( $a$ is the lattice spacing) is a hopping matrix element between nearest neighbors $\langle m, n\rangle$, and $\theta_{m n}=\frac{e}{\hbar c} \int_{m}^{n} \boldsymbol{A} \cdot d \boldsymbol{\ell}$ is an Aharonov-Bohm phase. $V_{m}$ is the disorder potential at site $m$, often assumed to be uniformly distributed over the interval $[-w / 2, w / 2]$. The disorder parameter $w$ determines the elastic mean free path, and in the Born approximation $k_{F} l \propto(w / t)^{-2}$. The Anderson model has become the standard model for describing single-particle dynamics of disordered mesoscopic systems. In $3 \mathrm{D}, w / t$ has a critical value above which all states become localized and the conductivity falls to zero, corresponding to a metal-insulator transition (Lee and Ramakrishnan, 1985). One-parameter renormalization-group analysis has shown that in $1 \mathrm{D}$ and $2 \mathrm{D}$ all states are localized. However, in 2D the localization length $\xi$ is exponentially large for weak disorder, and most states are extended over the dimension of the system.

Ballistic dots are often modeled as cavities. Billiard models are popular because their mathematical properties are best known and there are efficient methods to solve them numerically. By changing shape parameters 
in billiard models, it is possible to describe systems with classical motion ranging from integrable to fully chaotic. A good example is the conformal billiard (Robnik, 1983), whose shape is determined by the image of the unit circle in the complex $z$ plane under the conformal mapping $w(z)=\left(z+b z^{2}+c \mathrm{e}^{i \delta} z^{3}\right) / \sqrt{1+2 b^{2}+3 c^{2}}$.

\section{B. The semiclassical approach}

As a coherent phenomenon, transport in mesoscopic systems should be described by quantum mechanics. A classical treatment, on the other hand, has the advantage of physical intuition. The semiclassical approach is a bridge that seeks to describe quantum-mechanical phenomena in the language of classical physics. The semiclassical approach played an important role in the development of the mesoscopic theory of open ballistic dots where the approximation of non-interacting quasiparticles holds (Sec. IV). Applications to disordered systems have, for the most part, confirmed results derived earlier in the diagrammatic approach.

Most of the applications are based on an expansion of the Green function in terms of classical trajectories. The retarded Green function $G^{R}\left(\boldsymbol{r}, \boldsymbol{r}^{\prime} ; t\right) \equiv$ $\left\langle\boldsymbol{r}^{\prime}\left|e^{-i H t / \hbar}\right| \boldsymbol{r}\right\rangle$ propagates the particle from $\boldsymbol{r}$ at $t=0$ to $\boldsymbol{r}^{\prime}$ at a later time $t$. In Feynmann's path-integral representation, $G^{R}\left(\boldsymbol{r}, \boldsymbol{r}^{\prime} ; t\right)=\int_{\boldsymbol{x}(0)=\boldsymbol{r}}^{\boldsymbol{x}(t)=\boldsymbol{r}^{\prime}} D[\boldsymbol{x}] e^{i S[\boldsymbol{x}] / \hbar}$ is described as a functional integral over all trajectories $\boldsymbol{x}(t)$ that connect $\boldsymbol{x}(0)=\boldsymbol{r}$ to $\boldsymbol{x}(t)=\boldsymbol{r}^{\prime}$, where $S[\boldsymbol{x}]=$ $\int_{0}^{t} d \tau\left[m(d \boldsymbol{x} / d \tau)^{2} / 2-V(\boldsymbol{x})\right]$ is the action. In the limit $\hbar \rightarrow 0$ one can use the stationary phase approximation, leading to $\delta S=0$, Hamilton's variational principle for the classical trajectories $\boldsymbol{x}_{\alpha}$ between $(\boldsymbol{r}, 0)$ and $\left(\boldsymbol{r}^{\prime}, t\right)$. Small quantal fluctuations around each of the classical solutions $\boldsymbol{x}_{\alpha}$ are included by expanding the action to second order $S[\boldsymbol{x}] \approx S\left[\boldsymbol{x}_{\alpha}\right]+\delta^{2} S\left[\boldsymbol{x}_{\alpha}\right] / 2$ and doing the Gaussian integral. The result is Van Vleck's formula,

$$
G^{R}\left(\boldsymbol{r}, \boldsymbol{r}^{\prime} ; t\right) \approx \sum_{\alpha \in\left\{\boldsymbol{r}, \boldsymbol{r}^{\prime} ; t\right\}} A_{\alpha} e^{i S_{\alpha} / \hbar}
$$

where the sum is over all classical paths $\alpha$ between $(\boldsymbol{r}, 0)$ and $\left(\boldsymbol{r}^{\prime}, t\right)$ with action $S_{\alpha}=S\left[\boldsymbol{x}_{\alpha}\right]$ and amplitude $A_{\alpha}$ given by

$$
\begin{aligned}
& A_{\alpha}=\left(\frac{1}{2 \pi i \hbar}\right)^{\frac{d}{2}}\left|\operatorname{det}\left(-\frac{\partial^{2} S_{\alpha}}{\partial \boldsymbol{r}^{\prime} \partial \boldsymbol{r}}\right)\right|^{1 / 2} e^{-i \frac{\pi}{2} \nu_{\alpha}} \\
& S_{\alpha}=\int_{\boldsymbol{x}(0)=\boldsymbol{r}}^{\boldsymbol{x}(t)=\boldsymbol{r}^{\prime}}(\boldsymbol{p} \cdot d \boldsymbol{x}-H d t) .
\end{aligned}
$$

The classical action $S_{\alpha}\left(\boldsymbol{r}, 0 ; \boldsymbol{r}^{\prime}, t\right)$ is a function of the initial $(\boldsymbol{r}, 0)$ and final $\left(\boldsymbol{r}^{\prime}, t\right)$. In $d$ degrees of freedom, $-\partial^{2} S_{\alpha} / \partial \boldsymbol{r}^{\prime} \partial \boldsymbol{r}$ is a $d \times d$ matrix, and the integer phase index $\nu_{\alpha}$ is the number of its negative eigenvalues (equal to the number of conjugate points along the path). Since $-\partial S_{\alpha} / \partial \boldsymbol{r}=\boldsymbol{p}_{\alpha}$ is the initial momentum, this matrix can also be written as $\partial \boldsymbol{p}_{\alpha} / \partial \boldsymbol{r}^{\prime}$.

The energy representation of the retarded Green's function is the Fourier transform of Eq. (47). Doing the time integral by stationary phase, we find

$$
\begin{aligned}
G^{R}\left(\boldsymbol{r}, \boldsymbol{r}^{\prime} ; E\right) & =\int_{0}^{\infty} d t e^{i E t / \hbar} G^{R}\left(\boldsymbol{r}, \boldsymbol{r}^{\prime}, t\right) \\
& \approx \sum_{\alpha \in\left\{\boldsymbol{r}, \boldsymbol{r}^{\prime} ; E\right\}} \tilde{A}_{\alpha} e^{i \tilde{S}_{\alpha} / \hbar},
\end{aligned}
$$

where now the sum is over all classical paths $\alpha$ with energy $E$ that begin at $\boldsymbol{r}$ and end at $\boldsymbol{r}^{\prime}$. The modified action $\tilde{S}_{\alpha}=\tilde{S}_{\alpha}\left(\boldsymbol{r}, \boldsymbol{r}^{\prime} ; E\right)=S_{\alpha}+E t$ and amplitude $\tilde{A}_{\alpha}$ are

$$
\begin{aligned}
\tilde{A}_{\alpha} & =\frac{1}{i \hbar}\left(\frac{1}{2 \pi i \hbar}\right)^{\frac{d-1}{2}}\left|\operatorname{det}\left(\frac{\partial \boldsymbol{p}_{\alpha}}{\partial \boldsymbol{r}^{\prime}}\right)\right|^{1 / 2}\left|\frac{d T_{\alpha}}{d E}\right|^{1 / 2} e^{-i \frac{\pi}{2} \tilde{\nu}_{\alpha}} ; \\
\tilde{S}_{\alpha} & =\int_{r}^{r^{\prime}} \boldsymbol{p} \cdot d \boldsymbol{r}
\end{aligned}
$$

where $T_{\alpha}=\partial \tilde{S}_{\alpha} / \partial E$ is the duration of orbit $\alpha$, the derivative matrix $-\partial \boldsymbol{p}_{\alpha} / \partial \boldsymbol{r}^{\prime}$ is evaluated at $t=T_{\alpha}$, and $\tilde{\nu}_{\alpha}$ is a modified phase index (see, e.g., Reichel, 1992).

Equation (49) is the starting point of the semiclassical approximation for quantities that can be expressed in terms of Green's functions. For example, the density of states $\rho(E)=\sum_{i} \delta\left(E-E_{i}\right)$ can be written as $\rho(E)=-\pi^{-1} \int d \boldsymbol{r} \operatorname{Im} G^{R}(\boldsymbol{r}, \boldsymbol{r} ; E)$. Using Eq. (49) and integrating over $\boldsymbol{r}$ in the saddle-point approximation leads to a sum over periodic orbits (Gutzwiller 1967, 1969, 1970, 1971; Balian and Bloch, 1972). The level density is decomposed into a smooth average part (Weyl's term) and a fluctuating part: $\rho=\bar{\rho}+\rho_{\text {fluct }}$. In a fully chaotic system, the periodic orbits are isolated, and $\rho_{\text {fluct }}$ can be written in terms of Gutzwiller's trace formula (Gutzwiller, 1971)

$\rho_{\text {fluct }}(E)=\frac{1}{\pi \hbar} \sum_{\text {p.o. } \alpha} \frac{T_{\alpha}}{\left|\operatorname{det}\left(\tilde{M}_{\alpha}-I\right)\right|^{1 / 2}} \cos \left(\frac{\tilde{S}_{\alpha}}{\hbar}-\sigma_{\alpha} \frac{\pi}{2}\right)$,

where the sum is over periodic orbits $\alpha$. The phase $\sigma_{\alpha}$ is the Maslov index (containing the phase index $\tilde{\nu}_{\alpha}$ ) and $\tilde{M}_{\alpha}$ is a $(2 d-2)$-dimensional stability matrix of the orbit. $\tilde{M}_{\alpha}$ is a submatrix of the $2 d$-dimensional monodromy matrix $M_{\alpha}$ that describes the linear relation between a small change in the initial and final (i.e., after one period) $\delta \boldsymbol{r}$ and $\delta \boldsymbol{p}$.

\section{Spectral correlations in chaotic and disordered systems}

Spectral properties of metallic grains have long been of interest. Gor'kov and Eliashberg (1965) studied the elec- 
trical polarizability of small metallic grains by assuming RMT spectral fluctuations (see Sec. III.C). More recently, it became possible to do spectroscopy of lowlying states in quantum dots (Sivan et al., 1994). Here we discuss briefly the semiclassical calculation of spectral correlation in both chaotic and disordered systems. For reviews on chaos see, for example, Gutzwiller (1990) and Giannoni, Voros, and Zinn-Justin (1991). For a recent review of spectral correlations in disordered systems, see Dittrich (1996). In general, semiclassical methods are valid at energy scales that are large compared with the mean level spacing $\Delta$, namely, at time scales below the Heisenberg time $\tau_{H}$.

An important statistical measure of spectral correlations is the two-point correlation function of the density of states, measuring the correlations of $\rho$ at two different energies, $E$ and $E+\Omega$. Measuring energies in units of the mean level spacing (i.e., $\epsilon \equiv E / \Delta$ and $\omega \equiv \Omega / \Delta$ ), the correlator becomes dimensionless:

$$
K(\omega) \equiv \overline{\rho(\epsilon) \rho(\epsilon+\omega)}-\bar{\rho}^{2} .
$$

The Fourier transform $K(t)=\int d \omega K(\omega) e^{i \omega t}$ is known as the spectral form factor.

We first derive a semiclassical expression for $K(t)$ (Berry, 1985) in a chaotic system using the periodic orbit expansion (51). Measuring time in units of $\hbar / \Delta$ (so that the Heisenberg time is $\tau_{H}=2 \pi$ ) and using $\tilde{S}_{\alpha}(E+\Omega) \simeq \tilde{S}_{\alpha}(E)+\hbar T_{\alpha} \omega$, one finds $K(t) \sim(2 \pi)^{-1} \overline{\sum_{\alpha, \beta} \mathcal{A}_{\alpha} \mathcal{A}_{\beta} e^{\frac{i}{\hbar}\left(\tilde{S}_{\alpha}-\tilde{S}_{\beta}\right)} \delta\left[t-\left(T_{\alpha}+T_{\beta}\right) / 2\right]}$, where $\mathcal{A}_{\alpha} \equiv T_{\alpha} /\left|\operatorname{det}\left(\tilde{M}_{\alpha}-I\right)\right|^{1 / 2}$, and the average is over the energy $E$. In the diagonal approximation, only pairs of orbits with $\alpha=\beta$ are taken into account, and

$$
K_{\mathrm{sc}}(t) \sim(2 \pi)^{-1} \overline{\sum_{\alpha} \mathcal{A}_{\alpha}^{2} \delta\left(t-T_{\alpha}\right)} \approx|t| / 2 \pi .
$$

In the last step in deriving Eq. (53), we have used the classical sum rule of Hannay and Ozorio de Almeida (1984) for ergodic systems. This sum rule is valid for times that are long compared with a typical period of the short periodic orbits $\left(\sim \tau_{c}\right)$, but much shorter than the Heisenberg time. We shall see that this result is also the universal RMT result below the Heisenberg time. In deriving Eq. (53) we have assumed a system in which time-reversal symmetry is broken. For conserved timereversal symmetry we must also consider pairs of orbits that are time reversals of each other, and this will increase $K(t)$ by a factor of 2 .

The semiclassical approach to disordered metals can be found in Argaman, Imry and Smilansky (1993) and Montambaux (1997). For $t \ll \tau_{H}$, the form factor $K(t)$ can be related semiclassically to the return probability $P(t)=\left|\left\langle\boldsymbol{r}\left|e^{-i H t / \hbar}\right| \boldsymbol{r}\right\rangle\right|^{2}$ (which measures the probability that the electron will return to its original starting point $\boldsymbol{r}$ after time $t): K(t)=(2 \pi)^{-1} t P(t)$. For diffusive motion the classical return probability is calculated from

$$
P_{\mathrm{cl}}(t)=D(\boldsymbol{r}, \boldsymbol{r} ; t)=\sum_{\boldsymbol{q}} e^{-D q^{2} t} .
$$

In the case of conserved time-reversal symmetry there is an additional contribution from the constructive interference of orbits that are time-reversed pairs. We thus have $P(t)=(2 / \beta) P_{c l}(t)$, where $\beta=1$ for conserved time-reversal symmetry and $\beta=2$ for broken time-reversal symmetry. The Fourier transform of Eq. (54) is $P(\omega)=\sum_{\boldsymbol{q}}\left(-i \omega+D q^{2}\right)^{-1}$, and the semiclassical two-point correlation function is given by

$$
K_{s c}(\omega)=\frac{1}{\beta \pi^{2}} \operatorname{Im} \frac{\partial P}{\partial \omega}=-\frac{1}{\beta \pi^{2}} \operatorname{Re} \sum_{\boldsymbol{q}} \frac{1}{\left(-i \omega+D q^{2}+\gamma\right)^{2}},
$$

where an additional broadening $\gamma \sim \Gamma_{\phi}=\hbar / \tau_{\phi}$ is introduced to take into account the electron's finite coherence time (if $\tau_{\phi} \gg \tau_{H}$ one chooses $\gamma \sim \Delta$, since the semiclassical approximation breaks down at the Heisenberg time). Equation (55) was first derived by Altshuler and Shklovskii (1986) using diagrammatic techniques. The diagonal "classical" contribution is known as the diffuson, while the interference contribution (for conserved time-reversal symmetry) is described by the cooperon, obtained by summation of maximally crossed diagrams (Altshuler and Simons, 1995).

Inspecting Eq. (55), we can distinguish two regimes. For $\Omega=\omega \Delta<E_{c}$, the $\boldsymbol{q}=0$ diffusion mode dominates, and we obtain the ergodic limit where the electron samples the whole dot. On the other hand, for $\Omega \gg E_{c}$, the electron samples only a small fraction of the dot, and we can consider the limit of diffusion in an infinite system, where the summation in Eq. (54) over all modes $\boldsymbol{q}$ can be performed exactly to give $P_{c l}(t)=L^{d} /(4 \pi D t)^{d / 2}$. In these two limits it is found that (Braun and Montambaux, 1995; Montambaux, 1997)

$$
K(\omega) \approx\left\{\begin{array}{c}
-\operatorname{Re} \frac{1}{\beta \pi^{2}(\omega+i \gamma)^{2}} \quad\left(\text { for } 1 \ll \omega<g_{T}\right) \\
-\frac{1}{\beta \omega^{2}}\left(\frac{\omega}{g_{T}}\right)^{d / 2} \cos \left(\frac{\pi d}{4}\right) \quad\left(\text { for } \omega \gg g_{T}\right)
\end{array} .\right.
$$

For $1 \ll \omega<g_{T}, K(\omega)$ in Eq. (56) is universal. It coincides (for $\gamma=0$ ) with the semiclassical result of Berry (1985) in chaotic systems for $\omega$ below the ballistic $g_{T}$. In Sec. III.C we shall see that this universal semiclassical result describes the smooth part of the RMT prediction for $1 \ll \omega<g_{T}$. Random-matrix theory is nonperturbative and provides the exact universal results in a broader regime $\omega<g_{T}$ (i.e., not requiring $\omega \gg 1$ ). On the other hand, according to Eq. (56), the regime $\omega \gg g_{T}$ is nonuniversal and the correlator depends on dimensionality and size (through $g_{T}$ ). Although this AltshulerShklovskii nonuniversal power law was first predicted in 
1986, it was observed numerically in disordered metals only in 1995 (Braun and Montambaux, 1995).

\section{Conductance fluctuations in disordered metals}

A semiclassical approach to conductance fluctuations in disordered systems was discussed by Argaman (1996). The conductivity tensor of noninteracting electrons can be expressed in terms of Green's functions, and a semiclassical expression can be obtained using Eq. (49). In the diagonal approximation one recovers the energyaveraged classical conductivity $\bar{\sigma}$, i.e., Drude's formula. Quantum corrections result from interference of classical trajectories. Only pairs of trajectories that are related by symmetry can survive the averaging process. In particular, in systems with time-reversal symmetry we can consider the interference between a path that forms a closed loop and a path that follows a similar trace except in the loop segment, which it crosses in the opposite direction. These paths are time-reversed partners within the loop segment and their constructive interference leads to a decrease $\Delta \bar{\sigma}$ in the average conductivity, an effect known as weak localization or coherent backscattering. Quantum corrections to the conductance due to self-intersecting time-reversed paths were first noticed by Langer and Neal (1966) using diagrammatic methods. A semiclassical expression for the weak-localization correction can be found semiquantitatively by estimating the total number of closed loops. The probability for one closed orbit of period $t$ is the return probability $P(t)$. Since the initial point $\boldsymbol{r}$ can be anywhere along the path and the position of the electron is uncertain within $\lambda_{F}$ from its deterministic classical orbit, we multiply this probability by the volume of a tube of length $v_{F} t$ and thickness $\lambda_{F}$. We obtain the following estimate (for a large conductor of volume $V$ ):

$$
\Delta \bar{\sigma} / \bar{\sigma} \simeq-v_{F} \lambda_{F}^{d-1} V^{-1} \int_{\tau}^{\tau_{\phi}} d t P_{c l}(t)
$$

where $V^{-1} P_{\mathrm{cl}}(t)=(4 \pi D t)^{-d / 2}$ (see Sec. III.B.1). The above picture is valid only for times when the motion is diffusive $(t>\tau)$ and coherent $\left(t<\tau_{\phi}\right)$, hence the range of integration in Eq. (57). (Here we define the effective dimension $d$ of a conductor to be the number of dimensions for which the sample's extension is larger than $L_{\phi}$.) Integrating Eq. (57), we find that the weaklocalization correction to the average conductance per unit length is finite in $3 \mathrm{D}, \Delta \bar{G} \simeq-\left(e^{2} / h\right)\left[(3 / \pi)^{1 / 2} l^{-1}-\right.$ $\left.\left(D \tau_{\phi}\right)^{-1 / 2}\right]$, but increases logarithmically with $\tau_{\phi}$ in $2 \mathrm{D}$, $\Delta \bar{G} \simeq-\left(e^{2} / h\right) \ln \left(\tau_{\phi} / \tau\right)$, and linearly with $L_{\phi}$ in $1 \mathrm{D}$, $\Delta \bar{G}=-\left(e^{2} / h\right) 2 \pi\left(D \tau_{\phi}\right)^{1 / 2}$. The weak-localization correction to the conductivity was originally derived in the framework of disorder perturbation theory (Gorkov, Larkin and Khmelnitskii, 1979; see also Bergmann, 1984, and references therein; Khmelnitskii, 1984). For a quasiclassical approach to weak localization in disordered systems see Chakravarty and Schmid (1986). A magnetic field breaks time-reversal symmetry and can destroy the weak-localization correction (Altshuler et al., 1980).

The conductance in a mesoscopic conductor fluctuates as a function of Fermi energy and applied magnetic field. The magnitude of these fluctuations was found to be universal and of order $e^{2} / h$ (Altshuler, 1985; Lee and Stone 1985; Stone, 1985; Lee, Stone and Fukuyuma, 1987). This is the phenomenon of universal conductance fluctuations. Semiclassically, these fluctuations are related to the return probability through $\sigma^{2}(G) / \bar{G}^{2} \propto \int_{0}^{\infty} d t t P(t)$ (Argaman, 1996; Montambaux, 1997).

\section{The universal regime: random-matrix theory}

Random-matrix theory describes the statistical fluctuations in the universal regime (i.e., at energy scales below the Thouless energy). It was introduced by Wigner $(1951,1955,1957,1958)$ to explain the statistical fluctuations of neutron resonances in the compound nucleus. Rather than trying to explain individual eigenfunctions, RMT addresses questions about their statistical behavior. Its original justification was our lack of knowledge of the exact Hamiltonian; RMT assumes maximal ignorance regarding the system's Hamiltonian except that it must be consistent with the underlying symmetries. The theory proceeds to construct ensembles of Hamiltonians classified by their symmetry. Wigner's ideas were followed by those of Porter and Rosenzweig (1960) and Mehta and Gaudin $(1960,1961)$. Using group-theoretical methods developed by Wigner (1959), Dyson (1962a) showed that there are three classes of random-matrix ensembles. In a seminal paper entitled "The Threefold Way," Dyson (1962d) proved that the most general kind of matrix ensemble is a direct product of irreducible ensembles that belong to one of the three classes. Most of the early developments in the late 1950s and early 1960s are collected in Porter (1965). An early extensive review of RMT and its applications in nuclear physics was written by Brody et al. (1981). A detailed account of RMT can be found in the book by Mehta (1991).

Two major, independent developments in the early 1980 s considerably broadened the range of validity of RMT. One was the BGS conjecture (Bohigas, Giannoni, and Schmit, 1984) linking the quantal fluctuations in chaotic systems to RMT. Berry (1985) understood that the universality of RMT in chaotic systems holds for time scales that are longer than the shortest periodic orbits (i.e., the ergodic time). For time scales that are also much shorter than the Heisenberg time, the RMT results coincide with the semiclassical approach. However, RMT also provides the universal results at longer times where 
the diagonal semiclassical approximation fails. The BGS conjecture was confirmed in a large number of numerical studies. Applications of RMT to chaotic systems were reviewed by Bohigas (1991).

The second major development was Efetov's supersymmetry method (Efetov, 1983), which made possible a nonperturbative treatment of the single-particle disorder problem by mapping it onto the supersymmetric nonlinear $\sigma$ model. For weakly disordered systems and below the Thouless energy, this supersymmetric theory is in 0D and can be shown to be equivalent to RMT.

Random-matrix theory also seems to describe the statistical properties of interacting systems at high enough excitation (e.g., the compound nucleus), but its applicability to the ground-state properties of closed dots (where interactions are important) is not yet fully understood (see Sec. VI). Random-matrix theory has many applications in quantum physics; a comprehensive review emphasizing common concepts was written recently by Guhr, Müller-Groeling, and Weidenmüller (1998).

Section III.C.1 covers the most common ensembles of random matrices, the Gaussian ensembles. Section III.C.2 discusses the crossover ensembles, which are useful for describing the transition between different symmetry classes, e.g., the effects of a time-reversal symmetrybreaking magnetic field. In Sec. III.C.3, we generalize the Wigner-Dyson ensembles to Gaussian processes, an appropriate framework for describing the universal statistical properties of a system that depends on an external parameter. We end with another type of ensemble - Dyson's circular ensemble, suitable for describing statistical $S$ matrices and useful in the statistical theory of open quantum dots (Sec. IV.A).

\section{Gaussian ensembles}

The basic premise of RMT is that the statistical fluctuations of certain quantum systems can be described by an ensemble of $N \times N$ "random" matrices $H$. Since the matrix elements of the Hamiltonian of a physical system vanish between states with different good quantum numbers, it is only the Hamiltonian matrix in a subspace with fixed values of good quantum numbers that is assumed to be "random." Dyson (1962a, 1962d) found that there are only three types of ensembles, depending on the underlying space-time symmetries of the system. If the system is invariant under time reversal and under rotations, there is a basis where the Hamiltonian operator is represented by a real symmetric matrix (systems with time-reversal symmetry and broken rotational invariance but with integer total angular momentum also belong to this ensemble). If time-reversal symmetry is broken, irrespective of rotational invariance, then the Hamiltonian matrix is complex Hermitean. A third ensemble corresponds to systems that conserve time-reversal symmetry but are not rotationally invariant and have halfinteger total angular momentum. The matrix elements of such systems are real quaternion. A quaternion $q$ is a $2 \times 2$ matrix expressed in terms of a linear combination of the unit matrix $I$ and the three Pauli matrices $\sigma_{j}$, i.e., $q=a_{0} I+i \sum_{j} a_{j} \sigma_{j}$. The quaternion is real when the coefficients $a_{0}$ and $a_{j}$ are real. Each of the three classes has a different number of independent real components $\beta$ that characterizes a matrix element. We have $\beta=1,2$ and 4 for the ensembles of real symmetric, complex Hermitean, and real quaternion matrices, respectively.

The matrices that represent the same physical Hamiltonian in two different bases are related by a similarity transformation $H^{\prime}=W^{-1} H W$, where $W$ is the matrix connecting the two bases. We consider only transformations $W$ that preserve the "type" of the matrix $H$ that belongs to the given ensemble. For example, for $\beta=1$, the different bases can be chosen as "real" and the matrix $W$ must be orthogonal. Similarly, $W$ must be unitary for $\beta=2$ and symplectic (i.e., unitary matrices with real quaternion elements) for $\beta=4$. The three ensembles are thus called orthogonal $(\beta=1)$, unitary $(\beta=2)$ and symplectic $(\beta=4)$.

As a physical example, consider a single electron moving in a disorder or confining potential that is not rotationally invariant. If time-reversal symmetry and the electron spin are conserved, $\beta=1$ (since the motion is restricted to orbital space where the angular momentum assumes integer values). If time-reversal symmetry is broken, e.g., by a magnetic field, $\beta=2$. Finally, if timereversal symmetry is conserved but spin-rotation symmetry is broken, e.g., by strong spin-orbit scattering, $\beta=4$ (in this case the orbital and spin spaces are coupled and the total angular momentum has half-integer values).

We denote by $P(H) d H$ the probability of finding a matrix $H$ whose elements are in an interval $d H_{i j}$ around $H_{i j}$ (for $\beta=1, d H \equiv \prod_{i \leq j} d H_{i j}$ ). There are various methods of deriving the distribution $P(H)$. Porter and Rosenzweig (1960) require that the probability measure satisfy the following two properties (for the orthogonal case): (i) Invariance; $P\left(H^{\prime}\right)=P(H)$ under any similarity transformation with an orthogonal matrix $W$. Indeed, in complex or chaotic systems all bases chosen to represent the Hamiltonian should be statistically equivalent to each other. (ii) Statistical independence; all independent matrix elements are statistically independent, $P(H)=\prod_{i \leq j} P_{i j}\left(H_{i j}\right)$. The most general ensemble to satisfy both conditions is

$$
P(H) \propto e^{-\frac{\beta}{2 a^{2}} \operatorname{Tr} H^{2}} .
$$

The distribution (58) is manifestly invariant under any orthogonal transformation of $H$.

The measures for the other ensembles are similarly derived. We find the same expression (58) for all three 
ensembles. The quantity $\beta$ is introduced in the measure (58) for convenience only (the average level density becomes independent of $\beta$ ). The corresponding three Gaussian ensembles are called the Gaussian orthogonal ensemble (GOE), Gaussian unitary ensemble (GUE), and the Gaussian symplectic ensemble (GSE) for $\beta=1,2$, and 4 , respectively.

The Gaussian ensembles can also be defined by their first two moments. For the orthogonal $(\beta=1)$ and unitary $(\beta=2)$ cases we have

$$
\begin{aligned}
\overline{H_{i j}} & =0 ; \quad \overline{H_{i j} H_{k l}}=\frac{a^{2}}{2 \beta} g_{i j, k l}^{(\beta)} ; \\
g_{i j, k l}^{(\beta=1)} & =\delta_{i k} \delta_{j l}+\delta_{i l} \delta_{j k} ; \quad g_{i j, k l}^{(\beta=2)}=2 \delta_{i l} \delta_{j k} .
\end{aligned}
$$

In the orthogonal case, where all matrix elements are real, the variance of each diagonal element is $a^{2}$, while that of each off-diagonal element is $a^{2} / 2$. In the unitary case, the diagonal elements are real with variance $a^{2} / 2$, while the off-diagonal elements are complex and their real and imaginary parts each have a variance of $a^{2} / 4$.

Another derivation of the random-matrix ensembles was proposed by Balian (1968). His approach is based on information theory (Shannon, 1948), where the missing information (or entropy) associated with a distribution $P(H)$ is defined by $S[P(H)]=-\int d H P(H) \ln P(H)$ and measures the amount of missing information required to determine uniquely the system's Hamiltonian. Two constraints must be imposed on $P(H): \int d H P(H)=1$ (normalization) and $\int d H \operatorname{Tr} H^{2} P(H)=$ const (to ensure that the Hamiltonian's eigenvalues are bounded). We then choose the distribution $P(H)$ that is consistent with the constraints but is otherwise least biased, i.e., that maximizes the missing information. The solution is given by Eq. (58). This construction exemplifies the essence of RMT: it is the most "random" ensemble that is consistent with the underlying symmetries. The Gaussian ensembles lead to local correlations that are universally valid. In particular, their correlators coincide, after proper scaling, with the correlators of non-Gaussian ensembles (Brézin and Zee, 1993; Hackenbroich and Weidenmüller, 1995).

The distribution of eigenvalues and eigenvectors can be calculated from Eq. (58). We diagonalize the matrix H,

$$
W^{-1} H W=E
$$

and transform the variables $H_{i j}$ to new variables that consist of the $N$ eigenvalues $E_{\lambda}$ and a set of $\beta N(N-$ 1)/2 variables parametrizing the diagonalizing matrix $W$. This requires calculation of the Jacobian $J$ of the transformation. We present here a simple derivation of $J$ in the GOE case (Bohr and Mottelson, 1969). When $J \neq 0$, the transformation (60) is one to one and the eigenvectors must be uniquely determined from $H$. However, when two eigenvalues are degenerate, $E_{\lambda}=E_{\mu}$, this is not the case since the degenerate eigenvectors are determined only up to a linear combination, and therefore $J=0$. Furthermore, since the transformation (60) is linear in $E_{\lambda}, J$ is a polynomial of degree $N(N-1) / 2$ in the $E_{\lambda}$ 's. These properties of $J$ determine its dependence on the eigenvalues: $J \propto \prod_{\lambda<\mu}\left|E_{\lambda}-E_{\mu}\right|$. The general result for any of the three ensembles is $J \propto \prod_{\lambda<\mu}\left|E_{\lambda}-E_{\mu}\right|^{\beta}$, where the proportionality constant depends on the eigenvector parameters alone. We conclude that the eigenvalues are uncorrelated from the eigenvectors and are distributed according to

$$
P_{N}\left(E_{1}, E_{2}, \ldots, E_{N}\right) \propto\left(\prod_{\lambda<\mu}\left|E_{\lambda}-E_{\mu}\right|^{\beta}\right) e^{-\frac{\beta}{2 a^{2}} \sum_{\nu} E_{\nu}^{2}}
$$

\section{a. Spectral statistics}

The average level density in RMT is given by Wigner's semicircle $\bar{\rho}=\sqrt{2 a^{2} N-E^{2}} / \pi a^{2}$. Random-matrix theory is not expected to reproduce global level densities of realistic systems but only to describe the local fluctuations of the spectrum. To compare the statistical properties of a given spectrum with RMT, one first unfolds it, i.e., transforms it into one with constant average level density (Bohigas and Giannoni, 1984). An assumption implicit in most applications of RMT is that of ergodicity: the ensemble average is equivalent to the running average over a given spectrum. Given a physical system, one can collect statistics from different parts of the spectrum and then compare them with the RMT ensemble average.

There are several useful statistical measures of spectral fluctuations:

(i) Nearest-neighbor level-spacing distributions $P(s)$. Their asymptotic forms for large $N$ cannot be written in a simple form, but they are surprisingly well approximated by the simple expressions obtained for $N=2$ (Wigner's surmise)

$$
P_{\mathrm{WD}}(s)= \begin{cases}\frac{\pi}{2} s e^{-\frac{\pi}{4} s^{2}} & (\mathrm{GOE}) \\ \frac{32}{\pi^{2}} s^{2} e^{-\frac{4}{\pi} s^{2}} & (\mathrm{GUE}) \\ \frac{2^{18}}{3^{6} \pi^{3}} s^{4} e^{-\frac{64}{9 \pi} s^{2}} & (\mathrm{GSE})\end{cases}
$$

where the spacing $s$ is measured in units of $\Delta$. Equations (62) are often called the Wigner-Dyson distributions. The GOE and GUE distributions are shown in Fig. 9(a). Level repulsion is stronger in the GUE than in the GOE, as is seen from the small spacing behavior $P_{\mathrm{WD}}(s) \propto s^{\beta}$. 

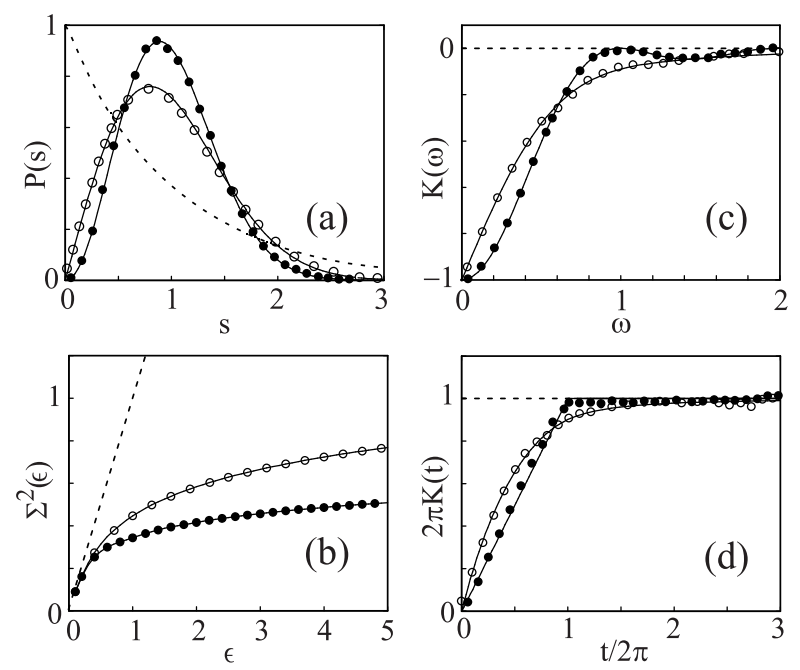

FIG. 9. Spectral measures of a metal in the diffusive regime, compared with the predictions of RMT. The circles are from numerical simulations of the Anderson model in its weakly disordered regime: open circles, without magnetic flux; solid circles, with magnetic flux. The solid lines are the predictions of RMT using GOE for conserved time reversal symmetry (no flux) and GUE for broken time-reversal symmetry (with flux).(a) Nearest-neighbor level-spacing distribution $\mathrm{P}(\mathrm{s})$; (b) number-variance statistics $\Sigma^{2}(\epsilon)$ defined by (64); (c) two-point level-density correlation function $K(\omega)$ defined by Eq. (52) (excluding a self-correlation $\delta$-function term); (d) the form factor $K(t)$, i.e., the Fourier transform of $K(\omega)$. The agreement with the RMT predictions is nearly perfect. The dotted lines correspond to the Poisson statistics where the energy levels are assumed to be random. Adapted from Braun and Montambaux (1995) and Montambaux (1997).

(ii) The $n$-point cluster functions $Y_{n}\left(\epsilon_{1}, \ldots, \epsilon_{n}\right)$ of $n$ levels $\epsilon_{\lambda}=E_{\lambda} / \Delta$. Dyson (1962b) defines the $n$-point correlation function $R_{n}\left(E_{1}, \ldots, E_{n}\right)=$ $[N ! /(N-n) !] \int\left(\prod_{i>n} d E_{i}\right) P_{N}\left(E_{1}, \ldots, E_{N}\right) \quad$ as the probability density of finding the $n$ levels $E_{1}, \ldots, E_{n}$ irrespective of the location of all other levels. $Y_{n}$ is the $n$ thorder cumulant constructed from the $R_{m}(m=1, \ldots, n)$. For example, the two-level cluster function is defined by $Y_{2}\left(\epsilon_{1}, \epsilon_{2}\right)=\Delta^{2}\left[R_{1}\left(E_{1}\right) R_{1}\left(E_{2}\right)-R_{2}\left(E_{1}, E_{2}\right)\right]$, and depends only on the energy difference $\omega \equiv \epsilon_{2}-\epsilon_{1}$. For the Gaussian ensembles (Dyson, 1970; Mehta, 1971)

$$
Y_{2}(\omega)=\left\{\begin{array}{c}
\left(\frac{\sin \pi \omega}{\pi \omega}\right)^{2}-\left[\operatorname{Si}(\pi \omega)-\frac{1}{2} \pi \operatorname{sgn}(\omega)\right] \\
\times\left[\frac{\cos \pi \omega}{\pi \omega}-\frac{\sin \pi \omega}{(\pi \omega)^{2}}\right] \\
\left(\frac{\sin \pi \omega}{\pi \omega}\right)^{2} \\
\left(\frac{\sin 2 \pi \omega}{2 \pi \omega}\right)^{2}-\operatorname{Si}(2 \pi \omega) \\
\times\left[\frac{\cos 2 \pi \omega}{2 \pi \omega}-\frac{\sin 2 \pi \omega}{(2 \pi \omega)^{2}}\right]
\end{array}\right.
$$

where $\operatorname{Si}(x)=\int_{0}^{x} d t \sin t / t$. It is also useful to transform to the time domain, where $b_{2}(t) \equiv \int_{-\infty}^{\infty} d \omega e^{2 \pi i \omega t} Y_{2}(\omega)$ is known as the two-level form factor (Brody et al., 1981).

The two-point level-density correlation function $K(\omega)$ defined by Eq. (52) is directly related to $Y_{2}$ by $K(\omega)=$ $\delta(\omega)-Y_{2}(\omega)$, while the associated spectral form factor is $K(t)=1-b_{2}(t / 2 \pi)$. The RMT result for times $t \ll$ $\tau_{H}=2 \pi$ is $K(t) \approx-(2 / \beta)(|t| / 2 \pi)$, in agreement with the diagonal semiclassical approximation (53). Similarly, for $\omega \gg 1$, Eqs. (63) give $K(\omega) \approx-1 / \beta \pi^{2} \omega^{2}$, in agreement with the semiclassical results [see, for example, Eq. (56) in a diffusive system below the Thouless energy]. The GOE and GUE results for $K(\omega)$ and $K(t)$ are shown in Figs. 9(c) and 9(d), respectively.

(iii) The number variance $\Sigma^{2}(\epsilon)={\overline{n^{2}(\epsilon)}}_{-\overline{n(\epsilon)}^{2}}$. This measures the variance of the number of levels $n(\epsilon)$ in an energy interval of length $\epsilon$. Since $n(\epsilon)=\int_{0}^{\epsilon} d \epsilon^{\prime} \rho\left(\epsilon^{\prime}\right)$ we have the relation

$$
\Sigma^{2}(\epsilon)=2 \int_{0}^{\epsilon} d \omega(\epsilon-\omega) K(\omega)=\epsilon-2 \int_{0}^{\epsilon} d \omega(\epsilon-\omega) Y_{2}(\omega) .
$$

The GOE and GUE number variances are shown in Fig. 9(b). For small $\epsilon, \Sigma^{2}(\epsilon) \approx \epsilon$, but of particular interest is the large- $\epsilon$ logarithmic behavior $\Sigma^{2}(\epsilon) \approx$ $\left(2 / \beta \pi^{2}\right) \ln \epsilon+$ const $+O\left(\epsilon^{-1}\right)$, where the constant is $\beta$ dependent. For $1 \ll \epsilon<g_{T}$ the diagrammatic (or semiclassical) result for the number variance in disordered metals coincides with the RMT results. However, for $\epsilon \gg g_{T}$, the diagrammatic result $\Sigma^{2}(\epsilon) \approx-\beta^{-1}\left(\epsilon / g_{T}\right)^{d / 2}$ is nonuniversal. The electron diffuses for a time $t$ that is much shorter than $\tau_{D}$ and covers only an area of linear dimension $\sqrt{D t}$ that is much smaller than $L$. The number variance is then proportional to the number of such "areas" $(L / \sqrt{D t})^{d} \sim\left(\epsilon / g_{T}\right)^{d / 2}$ contained within the total area of the system.

(iv) The Dyson-Mehta $\Delta_{3}$ statistics (Dyson and Mehta, 1963). A straight line is fitted by least squares to the staircase function (defined as the number of levels below a given energy) in an interval of length $\epsilon$. Here $\Delta_{3}(\epsilon)$ is the least-squared deviation from this best linear fit. The ensemble average of $\Delta_{3}$ is related to the number variance by (Pandey, 1979; Mehta, 1991)

$$
\bar{\Delta}_{3}(\epsilon)=\frac{2}{\epsilon^{4}} \int_{0}^{\epsilon} d \omega\left(\epsilon^{3}-2 \epsilon^{2} \omega+\omega^{3}\right) \Sigma^{2}(\omega) .
$$

In RMT, $\Delta_{3}$ starts as $\epsilon / 15$ and behaves asymptotically as $\bar{\Delta}_{3}(\epsilon) \approx\left(1 / \beta \pi^{2}\right) \ln \epsilon+$ const.

Gaussian orthogonal ensemble spectral correlations were found in the statistical analysis of the nuclear data ensemble, which consists of 1726 measured resonances in various compound nuclei (Haq, Pandey, and Bohigas, 1982; Bohigas, Haq, and Pandey, 1985). Random-matrix theory also describes the universal regime of disordered metals. This is confirmed in Fig. 9, which compares Anderson model calculations with and without magnetic flux (Dupuis and Montambaux, 1991; Braun and Montambaux, 1995) to RMT predictions. 


\section{b. Eigenfunction statistics}

In RMT, the probability distribution of an eigenvector's components $\psi_{i}(i=1, \ldots, N)$ is determined from the orthogonal (unitary) invariance of the ensemble (Brody et al., 1981),

$$
P\left(\psi_{1}, \psi_{2}, \ldots, \psi_{N}\right) \propto \delta\left(\sum_{i}\left|\psi_{i}\right|^{2}-1\right),
$$

where the metric is given by $\mathcal{D}[\boldsymbol{\psi}]=\prod_{i=1}^{N} d \psi_{i}$ for $\beta=1$ and $\prod_{i=1}^{N}\left(d \psi_{i}^{*} d \psi_{i} / 2 \pi i\right)$ for $\beta=2$.

To find the distribution of a finite number of components $\psi_{1}, \ldots, \psi_{\Lambda}$, we integrate Eq. (66) over all other $N-\Lambda$ components to find $P\left(\psi_{1}, \ldots, \psi_{\Lambda}\right) \propto$ $\left(1-\sum_{i=1}^{\Lambda}\left|\psi_{i}\right|^{2}\right)^{\beta(N-\Lambda) / 2-1}$. In the asymptotic limit $N \rightarrow \infty$, this distribution is a Gaussian $P(\boldsymbol{\psi}) \propto$ $\exp \left[-(\beta N / 2) \sum_{i=1}^{\Lambda}\left|\psi_{i}\right|^{2}\right]$. Of particular interest is the distribution of the intensity of a single component $y \equiv$ $\left|\psi_{i}\right|^{2}$,

$$
P(y)=\left(\frac{\beta}{2 \bar{y}}\right)^{\beta / 2} \frac{1}{(\beta / 2-1) !} y^{\beta / 2-1} e^{\frac{-\beta y}{2 y}},
$$

which is just the $\chi^{2}$ distribution in $\beta$ degrees of freedom. For the $\operatorname{GOE}(\beta=1)$ this is the Porter-Thomas distribution (Thomas and Porter, 1956) describing the neutron resonance widths in the compound nucleus - see, for example, Fig. 7(b).

More generally, for $n$ eigenvectors $(n \ll N), P\left(\boldsymbol{\psi}_{\lambda}\right) \propto$ $\prod_{\lambda=1}^{n} \exp \left[-(\beta N / 2) \sum_{i=1}^{\Lambda_{i}}\left|\psi_{i \lambda}\right|^{2}\right]$, and components that belong to different eigenvectors are to leading order uncorrelated.

\section{Crossover ensembles}

In some applications we are interested in the fluctuation properties of systems in the crossover regime between two different symmetries. The statistics in the crossover regime between GOE and GUE can be described by the Mehta-Pandey ensemble (Pandey and Mehta, 1983; Mehta and Pandey, 1983; Mehta, 1991; Bohigas, 1991)

$$
H=S+i \alpha A,
$$

where $S$ and $A$ are, respectively, symmetric and antisymmetric real matrices and $\alpha$ is a real parameter. The matrices $S$ and $A$ are uncorrelated and chosen from Gaussian ensembles of the same variance. Similar ensembles can be constructed to describe the crossover between the GUE and GSE or between the GOE and GSE (Mehta, 1991).

We are interested in the asymptotic limit $N \rightarrow \infty$, where the proper transition parameter is given by a typical symmetry-breaking matrix element measured in units of $\Delta$ (French and Kota, 1982; French et al., 1985, 1988): $\zeta=\left({\overline{H^{2}}}_{\text {break }}\right)^{1 / 2} / \Delta=\alpha \sqrt{N} / \pi$. For a fixed $\zeta$, the statistics of the ensemble (68) become independent of $N$ in the limit $N \rightarrow \infty$. The crossover parameter can also be expressed as $2 \pi \zeta=\sqrt{\tau_{H} / \tau_{\text {mix }}}$, where $\tau_{\text {mix }}$ is the mixing time defined in terms of the spreading width $\hbar / \tau_{\text {mix }}=2 \pi{\overline{H^{2}}}_{\text {break }} / \Delta$ of the time-reversal symmetrybreaking interaction (Pluhar̆ et al., 1994). The spectral statistics of the transition ensemble (around the middle of the spectrum) make the complete crossover for $\zeta \sim 1$.

In the transition ensembles, the eigenvalues and eigenvectors are no longer uncorrelated. The spectral statistics were derived by Pandey and Mehta (1983; see also Mehta and Pandey, 1983 and Mehta, 1991), but until recently less was known about the statistics of the eigenvectors in the transition ensembles. Fal'ko and Efetov (1994) used supersymmetry to derive the distribution of the wave function intensity, as well as the joint distribution of the wave function intensity at two distant spatial points (Fal'ko and Efetov, 1996). The recent work of van Langen, Brouwer, and Beenakker (1997) and Alhassid, Hormuzdiar, and Whelan (1998), based on earlier work of French et al. (1988), leads to a closed expression for the joint distribution of any finite number of the eigenvector's components.

The components of an eigenvector $\psi$ are complex: $\psi_{i}=$ $\psi_{i R}+i \psi_{i I}$. The eigenvector is determined only up to a phase $e^{i \theta}$, which can be fixed by rotating to a principal frame where (French et al., 1985, 1988)

$$
\sum_{i=1}^{N} \psi_{i R} \psi_{i I}=0 ; \quad \sum_{i} \psi_{i I}^{2} / \sum_{i} \psi_{i R}^{2} \equiv t^{2} .
$$

The eigenvector's components are distributed in the complex plane to form an ellipsoid whose semiaxes define the principal frame. The parameter $t$ in Eq. (69) $(0 \leq t \leq 1)$ determines the shape of this ellipsoid and is found to fluctuate in the crossover regime. Earlier theories (Zyczkowski and Lenz, 1991; Kogan and Kaveh, 1995; Kanzieper and Freilikher, 1996) ignored these fluctuations.

We now consider eigenvectors with a fixed shape parameter $t$. Under an orthogonal transformation $O$, the real and imaginary parts of $\psi$ transform like $\psi_{R} \rightarrow O \psi_{R}$ and $\psi_{I} \rightarrow O \psi_{I}$ and do not mix with each other. Consequently, Eq. (69) and the probability distribution of the transition ensemble (68) are invariant under an orthogonal transformation $O$. Thus the conditional probability distribution of the components of an eigenvector with a fixed "shape" $t$ is given by $P\left(\psi_{1}, \ldots, \psi_{N} \mid t\right) \propto$ $\delta\left(\sum_{i=1}^{N} \psi_{i R}^{2}-1 /\left(1+t^{2}\right)\right) \delta\left(\sum_{i=1}^{N} \psi_{i I}^{2}-t^{2} /\left(1+t^{2}\right)\right)$ $\times \delta\left(\sum_{i=1}^{N} \psi_{i R} \psi_{i I}\right)$. In the limit $N \rightarrow \infty$, the joint conditional distribution of a finite number of components $\Lambda \ll N$ becomes a Gaussian: 


$$
\begin{aligned}
& P\left(\psi_{1}, \ldots, \psi_{\Lambda} \mid t\right) \\
& \quad=\left(\frac{N}{2 \pi} \frac{1+t^{2}}{t}\right)^{\Lambda} \\
& \quad \times \exp \left(-N \frac{1+t^{2}}{2} \sum_{i=1}^{\Lambda} \psi_{i R}^{2}-N \frac{1+t^{2}}{2 t^{2}} \sum_{i=1}^{\Lambda} \psi_{i I}^{2}\right)
\end{aligned}
$$

The full distribution is computed by averaging the conditional distribution (70) over the distribution $P_{\zeta}(t)$ of the shape parameter: $P_{\zeta}\left(\psi_{1}, \ldots, \psi_{\Lambda}\right)=$ $\int_{0}^{1} d t P_{\zeta}(t) P\left(\psi_{1}, \ldots, \psi_{\Lambda} \mid t\right)$

It is still necessary to determine $P_{\zeta}(t)$. This can be done (Alhassid, Hormuzdiar, and Whelan, 1998) by calculating the distribution of the square of a single component using Eq. (70) and comparing it with the supersymmetry calculation by Fal'ko and Efetov (1994) of the wave-function-intensity distribution. It is found that

$$
\begin{aligned}
& P_{\zeta}(t)=\pi^{2} \frac{1-t^{4}}{t^{3}} \zeta^{2} e^{-\frac{\pi^{2}}{2} \zeta^{2}(t-1 / t)^{2}} \\
& \times\left\{\phi_{1}(\zeta)+\left[\frac{1}{4}\left(t+\frac{1}{t}\right)^{2}-\frac{1}{2 \pi^{2} \zeta^{2}}\right]\left[1-\phi_{1}(\zeta)\right]\right\},
\end{aligned}
$$

where $\phi_{1}(\zeta)=\int_{0}^{1} e^{-2 \pi^{2} \zeta^{2}\left(1-y^{2}\right)} d y$.

van Langen, Brouwer, and Beenakker (1997) calculated $P_{\zeta}(t)$ directly in the framework of RMT using a result of Sommers and Iida (1994) for the joint distribution of an eigenvalue and its associated eigenvector for a Hamiltonian in the ensemble (68). Rather than $t$, an equivalent "phase rigidity" parameter $\rho \equiv\left|\sum_{i} \psi_{i}^{2}\right|^{2}=$ $\left[\left(1-t^{2}\right) /\left(1+t^{2}\right)\right]^{2}$ is used.

The crossover distribution of a single component of the eigenfunction $y \equiv\left|\psi_{i}\right|^{2}=\psi_{i R}^{2}+\psi_{i I}^{2}$ is found from Eq. (70) to be (see also Fal'ko and Efetov, 1994)

$$
P_{\zeta}(y)=\left\langle\frac{1}{2 \bar{y}}\left(t+\frac{1}{t}\right) e^{-(t+1 / t)^{2} y / 4 \bar{y}} I_{0}\left(\frac{1-t^{4}}{4 t^{2}} \frac{y}{\bar{y}}\right)\right\rangle,
$$

where $I_{0}$ is the modified Bessel function of order zero, and $\langle\ldots\rangle$ denotes an average over the distribution in Eq. (71).

\section{Gaussian processes}

Consider a chaotic system that depends on an external parameter and whose symmetry class is the same for all values of the parameter. An interesting question is whether any universality can be found in the fluctuations of the system properties versus this parameter.

A semiclassical theory for a statistic that measures the correlation of energy levels at different values of an external parameter was proposed by Goldberg et al. (1991).
Szafer and Altshuler (1993) and Simons and Altshuler (1993a, 1993b) discovered that certain parametric spectral correlators of disordered systems are universal after an appropriate scaling of the parameter. Beenakker (1993) and Narayan and Shastry (1993) suggested that the parametric correlators can be studied in the framework of Dyson's Brownian-motion model (Dyson, 1962c).

The universality of eigenfunction correlators was shown by Alhassid and Attias (1995) through the generalization of Wigner-Dyson random-matrix ensembles to random-matrix processes describing the statistical properties of systems that depend on an external parameter. The supersymmetry approach was used to obtain the universal form of the oscillator strength-function correlator (Taniguchi, Andreev, and Altshuler, 1995). A Brownianmotion model for the parametric evolution of matrix elements of an operator between eigenstates was introduced by Wilkinson and Walker (1995).

We first discuss the generalization of the Gaussian ensembles to Gaussian processes. We assume a system whose Hamiltonian $H(x)$ depends on an external parameter $x$. The system is chaotic or weakly disordered for all values of $x$, and its underlying symmetry class is independent of $x$. To generalize the statistical description of RMT to include such a parametric dependence, we assume a Gaussian process $H(x)$ characterized by

$$
\overline{H_{i j}(x)}=0 ; \quad \overline{H_{i j}(x) H_{k l}\left(x^{\prime}\right)}=\frac{a^{2}}{2 \beta} f\left(x, x^{\prime}\right) g_{i j, k l}^{(\beta)},
$$

where the coefficients $g_{i j, k l}^{(\beta)}$ are defined in Eq. (59b). The process correlation function is assumed to be stationary and symmetric: $f\left(x, x^{\prime}\right)=f\left(\left|x-x^{\prime}\right|\right)$. It is normalized to $f(x, x)=1$ so that the matrix elements of $H(x)$ satisfy the Gaussian ensemble relations (59) for each fixed value of $x$.

The Gaussian process can also be defined in terms of its probability distribution

$$
\begin{aligned}
P\left[H\left(x^{\prime \prime}\right)\right] \propto & \exp \left\{-\left(\beta / 2 a^{2}\right) \int d x d x^{\prime}\right. \\
& \left.\times \operatorname{Tr}\left[H(x) K\left(x, x^{\prime}\right) H\left(x^{\prime}\right)\right]\right\},
\end{aligned}
$$

where the metric is $\mathcal{D} H \equiv \prod_{x} d H(x)$ with $d H$ being the usual Gaussian ensemble metric. Equation (74) is a direct generalization of the Gaussian ensemble measure (58) to include a parametric dependence of the randommatrix Hamiltonian. The kernel $K$ is the functional inverse of $f$.

Eq. (73) with $f\left(x-x^{\prime}\right)=\delta\left(x-x^{\prime}\right)$ describes a whitenoise random-matrix process $\Phi(x)$. Wilkinson (1989) introduced the process $H(x)=\int d x^{\prime} w\left(x-x^{\prime}\right) \Phi\left(x^{\prime}\right)$ in the study of the statistics of avoided crossing in chaotic systems. It is a Gaussian process with a correlation function $f(x)=\int d x^{\prime} w\left(x-x^{\prime}\right) w\left(-x^{\prime}\right)$.

Depending on the symmetry class of the matrices $H(x)$, there are three types of processes: the Gaussian 
orthogonal process $(\beta=1)$, the Gaussian unitary process $(\beta=2)$, and the Gaussian symplectic process $(\beta=4)$. A Gaussian process is characterized by a correlation function $f$, but in the asymptotic limit $N \rightarrow \infty$, only the short-distance behavior of $f$ (in parameter space) is important. We therefore expand $f$ to leading order in $x-x^{\prime}$ : $f\left(x-x^{\prime}\right) \approx 1-\kappa\left|x-x^{\prime}\right|^{\eta}$, where $\kappa$ and $\eta>0$ are constants, and classify the Gaussian processes according to the value of $\eta$.

Of particular interest are two-point parametric correlators of observables $O(x): \quad c_{\mathcal{O}}\left(x-x^{\prime}\right)=$ $\overline{\delta O(x) \delta O\left(x^{\prime}\right)} /\left[\sigma(O(x)) \sigma\left(O\left(x^{\prime}\right)\right)\right]$, where $\delta O(x)=O(x)-$ $\bar{O}(x)$ and $\sigma^{2}(O(x))=\overline{(\delta O(x))^{2}}$. To calculate $c_{\mathcal{O}}\left(x-x^{\prime}\right)$ we only need to know the joint distribution of $H(x)$ and $H\left(x^{\prime}\right)$. The Gaussian process has the useful property that the joint distribution of any finite number of matrices $H(x), H\left(x^{\prime}\right), H\left(x^{\prime \prime}\right), \ldots$ is Gaussian. In particular (Attias and Alhassid, 1995)

$$
\begin{aligned}
P\left[H(x), H\left(x^{\prime}\right)\right] & \propto \exp \left\{-\left(\beta / 2 a^{2}\right) \operatorname{Tr}\left[H(x)^{2}+H\left(x^{\prime}\right)^{2}\right.\right. \\
& \left.\left.-2 f H(x) H\left(x^{\prime}\right)\right] /\left(1-f^{2}\right)\right\}
\end{aligned}
$$

where $f \equiv f\left(x-x^{\prime}\right)$.

The Gaussian process parametric correlators depend on $a, f$, and $N$, but become universal upon an appropriate scaling of $x$. The dependence on $a$ is eliminated by unfolding the energies $E_{i} \rightarrow \epsilon_{i}=E_{i} / \Delta$. To eliminate the dependence on $f$, we calculate the mean-squared para-

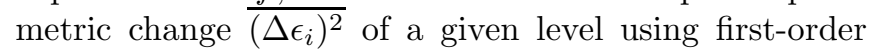
perturbation theory,

$$
\overline{\Delta \epsilon_{i}^{2}}=D_{\epsilon}|\Delta x|^{\eta}+\mathcal{O}\left(|\Delta x|^{2 \eta}\right),
$$

where $D_{\epsilon}=\lim _{\Delta x \rightarrow 0} \overline{\Delta \epsilon_{i}^{2}} / \Delta x^{\eta}=4 N \kappa / \pi^{2} \beta$. Equation (76) suggests that the energy levels undergo short-range "diffusion" as a function of the parameter $x$ (characterized by an exponent $\eta$ ), with $D_{\epsilon}$ playing the role of the diffusion constant. For a Gaussian process with $\eta<2$, the levels show irregular behavior as a function of $x$ which becomes smooth in the limit $\eta=2$. This is demonstrated in Fig. 10(a). Gaussian processes with $\eta=2$ are the only differentiable Gaussian processes (Attias and Alhassid, 1995); namely, they have the property that almost every one of their members has a continuous derivative $d H / d x$. Since in most physical applications the Hamiltonian is an analytic function of its parameter, we are interested only in differentiable Gaussian processes, i.e., $\eta=2$, and in the following we assume $\eta=2$. Equation (76) suggests the scaling (Simons and Altshuler, 1993a, 1993b)

$$
x \rightarrow \bar{x} \equiv \sqrt{D_{\epsilon}} x=\left[{\overline{\left(\partial \epsilon_{i} / \partial x\right)^{2}}}^{1 / 2} x,\right.
$$

under which the Gaussian process correlation function becomes independent of the non-universal constant $\kappa$ : $f \approx 1-\left(\beta \pi^{2} / 4 N\right)\left|\bar{x}-\bar{x}^{\prime}\right|^{2}$. Analytic calculations of certain correlators as well as numerical simulations support the conjecture that, for large $N$, all two-point correlators depend on $N$ and $f$ only through the combination $N(1-f)=\left(\beta \pi^{2} / 4\right)\left|\bar{x}-\bar{x}^{\prime}\right|^{2}$. We conclude that all parametric correlators are universal as a function of $\left|\bar{x}-\bar{x}^{\prime}\right|$. The universality can also be demonstrated by relating the Gaussian process to Dyson's Brownian motion model (Mitchell, Alhassid, and Kusnezov, 1996). The scaled $\bar{x}$ measures $x$ in units of the average parametric distance between avoided level crossings.
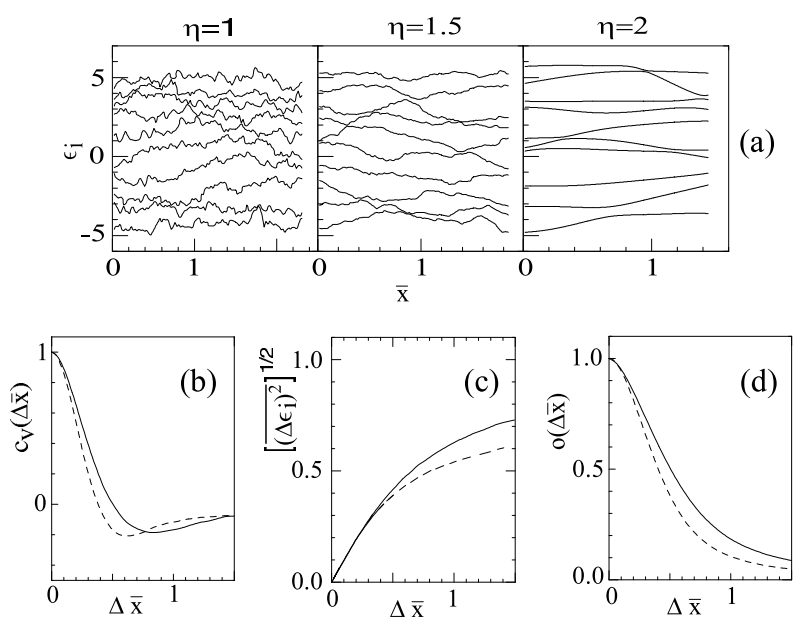

FIG. 10. Gaussian processes and parametric correlators: (a) typical parametric variation of the energy levels of Gaussian processes (73) with correlation functions $f(x)=\exp \left(-|x|^{\eta}\right)$ as a function of the scaled parameter $\bar{x}$. Shown are processes with $\eta=1,1.5$ and $2 . \quad \eta=2$ is the limit where the parametric variation of the energy levels becomes smooth. Panels (b) - (d) show three parametric correlators in the GOE (solid lines) and GUE (dashed lines) symmetries: (b) the level-velocity correlator $c_{v}(\Delta \bar{x})$; (c) the level-diffusion correlator $\left[\overline{\left(\Delta \epsilon_{i}\right)^{2}}\right]^{1 / 2} ;$ and $(\mathrm{d})$ the wave-function-overlap parametric correlator $o(\Delta \bar{x})$ of Eq. (79). The correlators are calculated using the Gaussian process (78). The level-velocity correlator coincides with the one calculated by Simons and Altshuler (1993a, 1993b) in the Anderson model. Adapted from Attias and Alhassid (1995).

Since the parametric correlators are universal, any Gaussian process can be used to calculate them. A particularly simple Gaussian process is given by (Austin and Wilkinson, 1992; Alhassid and Attias, 1995)

$$
H(x)=H_{1} \cos x+H_{2} \sin x,
$$

where $H_{1}$ and $H_{2}$ are two independent Gaussian matrices that belong to the appropriate symmetry. The process (78) is characterized by $f\left(x-x^{\prime}\right)=\cos \left(x-x^{\prime}\right)$, and is therefore an $\eta=2$ process. It is stationary and particularly useful for numerical simulations.

Several spectral parametric correlators were calculated and their universality demonstrated in models of disordered dots as well as chaotic dots. The correlator 
$c_{v}\left(x-x^{\prime}\right)$ of the level velocity $v_{\lambda}(x) \equiv \partial E_{\lambda} / \partial x$ was calculated by Simons and Altshuler (1993a, 1993b) using the Anderson model. Its universal form, calculated using the Gaussian process (78), is shown in Fig. 10(b) for both the orthogonal (solid line) and unitary (dashed line) symmetries. The Gaussian unitary process correlator decorrelates faster than the Gaussian orthogonal process correlator. Also shown in Fig. 10(c) is the leveldiffusion correlator $\left[\overline{\left(\Delta \epsilon_{i}\right)^{2}}\right]^{1 / 2}=\left[\overline{\left(\epsilon_{i}(x)-\epsilon_{i}\left(x^{\prime}\right)\right)^{2}}\right]^{1 / 2}$, describing the root mean square (rms) of the change of a given energy level over a finite parametric distance $\Delta \bar{x}$ (Attias and Alhassid, 1995). The level-density correlator $k(\omega, \Delta x) \equiv \overline{\delta \rho(\epsilon, x) \delta \rho(\epsilon+\omega, x+\Delta x)}$, where $\rho(\epsilon, x)=\sum_{\lambda} \delta\left(\epsilon-\epsilon_{\lambda}(x)\right)\left[\epsilon_{\lambda}\right.$ are the unfolded energy levels measured in units of $\Delta$ ], can be calculated in closed form using the supersymmetry method (Simons and Altshuler, 1993a, 1993b).

Parametric correlators that depend on the eigenfunctions, such as the averaged parametric overlap correlator (Alhassid and Attias, 1995; Attias and Alhassid, 1995) and the strength-function correlator (Taniguchi, Andreev, and Altshuler, 1995), were also found to be universal. The overlap correlator measures the squared overlap of an eigenfunction at different values of the external parameter:

$$
o\left(x-x^{\prime}\right)=\overline{\left|\left\langle\psi_{\lambda}(x) \mid \psi_{\lambda}\left(x^{\prime}\right)\right\rangle\right|^{2}} .
$$

This correlator was calculated numerically using the Gaussian process (78) and is shown in Fig. 10(d). The wave functions are observed to decorrelate faster in the unitary case. This parametric overlap correlator is well fit by a Lorentzian in the orthogonal case $(\beta=1)$ and by a squared Lorentzian in the unitary case $(\beta=2)$ :

$$
o\left(\bar{x}-\bar{x}^{\prime}\right) \approx\left[\frac{1}{1+\left(\bar{x}-\bar{x}^{\prime}\right)^{2} / \alpha_{\beta}^{2}}\right]^{\beta},
$$

with $\alpha_{1}=0.48 \pm 0.03$ in the orthogonal case and $\alpha_{2}=$ $0.64 \pm 0.04$ in the unitary case.

\section{Circular ensembles}

The Gaussian ensembles are well suited to the Hamiltonian approach, in which the Hamiltonian is assumed to be "random" and the statistics of various physical quantities of interest are then calculated from their relation to the Hamiltonian. However, transport properties of electrons through quantum dots can be related directly to the $S$ matrix (see Sec. II.B), and it is sometimes possible to derive the fluctuations of the conductance by assuming the $S$ matrix to be the fundamental "random" object (see Sec. IV.A). The $S$ matrix associated with a Hermitian Hamiltonian is unitary. Random-matrix ensembles of unitary matrices are the circular ensembles introduced by Dyson (1962a).

There are three types of circular ensembles: orthogonal, unitary, and symplectic. For systems with timereversal invariance, the $S$ matrix is symmetric unitary, while for systems with broken time-reversal symmetry, the $S$ matrix is an arbitrary unitary matrix. Finally, the symplectic ensemble is composed of self-dual unitary quaternion $S$ matrices (Mehta, 1991). The measure of the circular ensembles is uniform over the unitary group of $S$ matrices, subject to the consistency condition that the symmetry properties of the $S$ matrix are preserved. More explicitly, the measure is required to satisfy

$$
d \mu(S)=d \mu(U S V)
$$

for any unitary matrices $U$ and $V$ that preserve the symmetry properties as $S$ under the transformation $S \rightarrow$ $U S V$. The measure satisfying Eq. (81) is known as the Haar measure.

A unitary matrix $S$ that belongs to any of the three circular ensembles can be diagonalized $U^{-1} S U=E$, where the eigenvector matrix $U$ is orthogonal, unitary, or symplectic for $\beta=1,2$, or 4 , respectively. The eigenvalues $e^{i \theta_{i}}$ define the eigenphases of the $S$ matrix. The eigenvector and eigenvalue distributions are uncorrelated, and

$$
P\left(\theta_{1}, \ldots, \theta_{N}\right) \propto \prod_{i \leq j}\left|e^{i \theta_{i}}-e^{i \theta_{j}}\right|^{\beta},
$$

where the measure is $\prod_{j} d \theta_{j}$. In the symplectic case the eigenvalues are doubly degenerate and only the nondegenerate values are taken in Eq. (82). The density of the eigenphases is uniform on the circle, and there is no need for unfolding. In the limit $N \rightarrow \infty$, various spectral correlations and cluster functions coincide with those of the Gaussian ensembles.

\section{The supersymmetry method}

The supersymmetry method provides a technique for calculating ensemble averages over products of Green's functions, where the Hamiltonian is either a random matrix or describes a single particle in a random potential. This is a nontrivial problem since the random quantities appear in the denominator. The method maps the original problem - after performing the ensemble average - onto a field-theoretical model, the supersymmetric nonlinear $\sigma$ model. For random matrices, the $\sigma$ model is in $0 \mathrm{D}$, while for a weakly disordered system in $d$ dimensions, the $\sigma$ model is in $d$ dimensions. However, for energy scales below the Thouless energy, it reduces to the 0D $\sigma$-model, explaining the RMT universality in weakly disordered systems. The supersymmetry method was introduced for disordered systems by Efetov (1983), and its use for random matrices was discussed by Verbaarschot, 
Weidenmüller, and Zirnbauer (1985). A variety of physical applications are included in a review by Altshuler and Simons (1995). A book by Efetov (1997) discusses the method and its numerous applications. The method was used extensively in the calculation of spectral and eigenfunction statistics in disordered systems; see a recent review by Mirlin (2000).

We first describe briefly the supersymmetry method for averaging over random-matrix ensembles. The starting point is to write the Green's function as a multidimensional Gaussian integral where the ensemble average is straightforward. In general, the inverse of a matrix $K$ can be written as $\left(K^{-1}\right)_{\lambda \mu}=(\operatorname{det} K) \int d \boldsymbol{s} s_{\lambda} s_{\mu}^{*} e^{-\boldsymbol{s}^{\dagger} K \boldsymbol{s}}$, where $s_{\nu}$ are commuting complex variables and $d s \equiv$ $\prod_{\nu}\left(d s_{\nu}^{*} d s_{\nu} / 2 \pi i\right)$. The determinant of $K$ can also be written as a Gaussian integral: $\operatorname{det} K=\int d \boldsymbol{\chi} e^{-\chi^{\dagger} K \boldsymbol{\chi}}$, but over Grassmanian (anticommuting) variables $\chi_{\nu}$ with $d \chi \equiv \prod_{\nu}\left(d \chi_{\nu}^{*} d \chi_{\nu}\right)$. Combining both representations, we can write the inverse of a matrix as a pure Gaussian integral over both commuting and anti-commuting variables:

$$
\left(K^{-1}\right)_{\lambda \mu}=\int d \boldsymbol{s} d \chi s_{\lambda} s_{\mu}^{*} e^{-s^{\dagger} K s-\chi^{\dagger} K \chi} .
$$

Equation (83) can be used to express the advanced and retarded Green's functions of an $N \times N$ Hamiltonian matrix $H$ as a Gaussian integral in the set $(\boldsymbol{s}, \boldsymbol{\chi})$ by choosing $K=\mp i\left(E^{ \pm}-H\right)$. In the GOE case the quadratic form in the exponent of Eq. (83) is rewritten by doubling the number of components (the doubling is not needed for the GUE case):

$$
\begin{aligned}
G\left(E^{ \pm}\right)= & \mp i \int d \boldsymbol{\Psi}\left(s s^{\dagger}\right) \\
& \times \exp \left\{ \pm \frac{i}{2} \boldsymbol{\Psi}^{\dagger}\left[\left(E^{ \pm}-H\right) \times I_{4}\right] \Psi\right\} .
\end{aligned}
$$

Here $\boldsymbol{\Psi} \equiv\left(\boldsymbol{s}, \boldsymbol{s}^{*}, \boldsymbol{\chi}, \boldsymbol{\chi}^{*}\right)^{T}$ is a $4 N$-dimensional vector and the measure is $d \boldsymbol{\Psi} \equiv d \boldsymbol{s} d \boldsymbol{\chi}$, while $M \otimes I_{n}$ denotes a block diagonal matrix with n blocks $M$.

Equation (84) can be generalized to products of Green's functions by introducing a separate vector $\boldsymbol{\Psi}_{j}$ for each Green's function. We describe below the calculation of quantities that involve the product of two Green's functions at two energies $E$ and $E^{\prime}$ and for Hamiltonians $H(x)$ and $H\left(x^{\prime}\right)$ taken at two different values of an external parameter. For example, the evaluation of the parametric level-density correlator $k[(E-$ $\left.\left.E^{\prime}\right) / \Delta, x-x^{\prime}\right]$ requires the calculation of terms like $C_{k} \equiv$ $\overline{\operatorname{Tr} G\left(E^{-}, x\right) \operatorname{Tr} G\left(E^{\prime+}, x^{\prime}\right)}$. We have

$$
\begin{aligned}
& C_{k}=\int d \boldsymbol{\Psi} p_{k}(\boldsymbol{\Psi}) \\
& \times \exp \left[\frac{i}{2} \boldsymbol{\Psi}^{\dagger}(-E \Lambda+\Omega / 2+i \delta) \boldsymbol{\Psi}\right] \overline{\exp \left(\frac{i}{2} \boldsymbol{\Psi}^{\dagger} \Lambda \mathcal{H} \boldsymbol{\Psi}\right)},
\end{aligned}
$$

where $\boldsymbol{\Psi} \equiv\left(\begin{array}{l}\boldsymbol{\Psi}_{1} \\ \boldsymbol{\Psi}_{2}\end{array}\right)$ is an $8 N$-dimensional vector, and $\mathcal{H}$ and $\Lambda$ are $8 N \times 8 N$ matrices. Here $\mathcal{H}$ has a $2 \times 2$ block-diagonal form, where the two diagonal blocks are $H(x) \otimes I_{4}$ and $H\left(x^{\prime}\right) \otimes I_{4}$. Moreover, $\Lambda$ has a similar structure with diagonal blocks of $I_{N} \otimes I_{4}$ and $-I_{N} \otimes I_{4}$. We have also defined $\bar{E}=\left(E+E^{\prime}\right) / 2, \Omega=E-E^{\prime}$, and $p_{k}(\boldsymbol{\Psi}) \equiv\left(\boldsymbol{s}_{1}^{\dagger} \boldsymbol{s}_{1}\right)\left(\boldsymbol{s}_{2}^{\dagger} \boldsymbol{s}_{2}\right)$. The calculation of Eq. (85) can be mapped onto the 0D supersymmetric nonlinear $\sigma$ model as follows:

(i) The average in Eq. (85) is taken using the secondorder cumulant in the exponent. We obtain an integral representation of $C_{k}$ with an action quartic in $\boldsymbol{\Psi}$.

(ii) The action is converted to one that is quadratic in $\mathbf{\Psi}$ by introducing an auxiliary integration variable $\sigma$ that is an $8 \times 8$ graded matrix (the Hubbard-Stratonovich transformation).

(iii) The Gaussian integral over $\boldsymbol{\Psi}$ is done exactly, leading to an effective action in $\sigma$.

(iv) The $\sigma$ integral is evaluated by the saddle-point method in the limit $N \rightarrow \infty$ and $\Delta \propto 1 / N \rightarrow 0$ (the quantities $\omega \propto \Omega / \Delta, \tilde{\delta} \propto \delta / \Delta$, and $\left|\bar{x}-\bar{x}^{\prime}\right| \propto N^{1 / 2}\left|x-x^{\prime}\right|$ are all kept constant in this limit). This leads to a nonlinear equation for $\sigma$ that is a supersymmetric generalization of the nonlinear $\sigma$ model.

(iv) The solutions $Q$ of the saddle-point equation form a manifold (satisfying $Q^{2}=I$ ). Here $C_{k}$ can be expressed as an integral over this saddle-point manifold,

$$
C_{k}=\int d Q p_{k}(Q) \exp [-\mathcal{F}(Q)],
$$

where the "free energy" is given by

$$
\begin{aligned}
\mathcal{F}(Q)= & i(\pi \omega / 4+i \tilde{\delta}) \operatorname{Trg}(Q \Lambda) \\
& -\left(\pi^{2} / 64\right)\left|\bar{x}-\bar{x}^{\prime}\right|^{2} \operatorname{Trg}\left([Q, \Lambda]^{2}\right)
\end{aligned}
$$

and $\operatorname{Trg}$ is a supertrace. The integration over $Q$ is performed using a parametrization of the saddle-point manifold by Efetov (1983). The integration can be done over all but three commuting variables that appear in the final analytic result for $k(\omega, x)$; see Simons and Altshuler, 1993a, 1993b.

The supersymmetry method for disordered systems follows along similar lines except that the discrete label $n$ $(n=1, \ldots, N)$ of the supervector $\boldsymbol{\Psi}$ becomes the continuous spatial label $\boldsymbol{r}$. The auxiliary supermatrices in the Hubbard-Stratonovich transformation acquire a spatial dependence $\sigma(\boldsymbol{r})$. The saddle-point approximation requires a large parameter $-k_{F} l$, which plays a role analogous to $N$ in RMT. By minimizing the action one finds a spatially uniform saddle point $Q_{0}=\Lambda$ that has the same form as in RMT. However, the saddle-point manifold now consists of matrix fields $Q(\boldsymbol{r})$ that describe longwavelength fluctuations. By expanding the action around the saddle point to leading order in $1 / k_{F} l$, we find (for 
the case without an external parametric dependence of the Hamiltonian) a free energy of the form

$\mathcal{F}[Q]=\frac{\pi \nu}{8} \int d \boldsymbol{r}\left[\hbar D \operatorname{Trg}(\nabla Q(\boldsymbol{r}))^{2}+2 i \Omega \operatorname{Trg}(Q \Lambda)\right]$,

where $D$ is the classical diffusion coefficient and $\nu$ is the density of states (per unit volume). The physical quantities are evaluated according to expressions of the form of Eq. (86) but where now the integration is over $d Q(\boldsymbol{r})$ at each $\boldsymbol{r}$.

The gradient term in Eq. (88) is of the order of the Thouless energy $E_{c}=\hbar D / L^{2}$. In the limit $\Omega \ll E_{c}$, one can ignore the $\boldsymbol{r}$ dependence of $Q$ and the gradient term vanishes. In this case, the volume integration of the second term in Eq. (88) gives exactly the first term in the RMT free energy (87). Deviations from the universal behavior are important when $\Omega$ exceeds the Thouless energy: the electron does not have enough time to diffuse to the boundaries and "senses" the dimensionality of the system. Such nonuniversal corrections were derived by Andreev and Altshuler (1995) for the asymptotic spectral correlator $K(\omega)$ in terms of the nonzero eigenvalues of the diffusion operator.

A supersymmetric $\sigma$ model for ballistic chaotic systems was suggested by Muzykantskii and Khmelnitskii (1995), using disorder averaging when $l>L$, and by Andreev et al. (1996) using energy averaging in a pure Hamiltonian system. The eigenvalues of the diffusion operator in the disordered case are replaced by those of the Perron-Frobenius operator in the ballistic case (Agam, Altshuler, and Andreev, 1995). The first nonzero eigenvalue sets the scale for the ergodic time above which we expect the RMT universality. However, the mathematical difficulties involved in implementing this approach seem so far to be insurmountable. Similar methods were used to investigate spectral and wave function fluctuations in billiards with diffusive surface scattering (Blanter, Mirlin, and Muzykantskii, 1998).

\section{MESOSCOPIC FLUCTUATIONS IN OPEN DOTS}

In this section we apply the methods of Sec. III to open quantum dots, where there are usually several conducting channels in each lead, and the conductance is typically much larger than $e^{2} / h$. Open dots with a large number of channels are characterized by many overlapping resonances, i.e., $\bar{\Gamma} \gg \Delta$, where $\bar{\Gamma}$ is an average width of a resonance level in the dot. When the single-electron dynamics in the dot are chaotic and the electron spends enough time in the dot before it escapes (i.e., $\tau_{\text {escape }} \gg \tau_{c}$ ), the conductance exhibits universal mesoscopic fluctuations as a function of gate voltage or magnetic field, independent of the dot's size and shape. However, the fluctuations do depend on the number of modes in the leads and their transmission coefficients. In the limit of a large number of open channels, the fluctuations become similar to the universal conductance fluctuations known from disordered conductors. In the universal regime, RMT can be used to characterize the conductance fluctuations. For a comprehensive review of the random-matrix theory of quantum transport, including applications to open dots, see Beenakker (1997).

The semiclassical approximation becomes useful in the limit of a large number of modes in the leads. It can predict the magnitude of certain dynamical quantities that cannot be calculated in RMT, e.g., the correlation length of the conductance fluctuations versus magnetic field. But the semiclassical approach is not suitable for dots with fewer than $\sim 3$ modes per lead, or for calculating quantities such as the conductance distribution. For reviews of the semiclassical approach to transport in open ballistic microstructures, see Baranger, Jalabert, and Stone (1993b), Stone (1995), and Baranger (1998). Our presentation in this Section integrates the randommatrix and semiclassical approaches to open dots.

The experimental results, while confirming the expected phenomena, disagree quantitatively with theory. The main reason is that the coherence length of the electron is finite at any nonzero temperature. When dephasing as well as thermal smearing are included in the statistical approach, good agreement is found between theory and experiment. This point was nicely demonstrated in a recent experiment (Huibers, Switkes, Marcus, Brouwer, et al., 1998) in dots with single-mode leads.

Although the quantum dots used in experiments are high-mobility ballistic structures, many of the derivations in this section are also applicable to diffusive dots. Indeed, as long as $\tau_{\text {escape }} \gg \tau_{D}$, the same RMT universality predicted in ballistic dots is expected for a diffusive dot.

Section IV.A presents two random-matrix approaches to conductance fluctuations in open dots, while Sec. IV.B describes the semiclassical approach. In Sec. IV.C we quantify the mesoscopic fluctuations of the conductance in open dots, including the conductance distributions, weak-localization effect, and fluctuations versus energy (Ericson fluctuations) or versus an experimentally controllable parameter. Finally, Sec. IV.E discusses dephasing and its effects on the mesoscopic fluctuations. Throughout the Section we include comparison to experimental results, with an emphasis on more recent results. Additional experimental results can be found in the review of Westervelt (1998).

\section{A. The random-matrix approach}

Historically, the random-matrix approach to scattering proceeded along two main directions: the Hamiltonian approach, in which the system's Hamiltonian is assumed 
to belong to a Gaussian ensemble (Sec. III.C.1), and the $S$-matrix approach, in which the $S$ matrix itself is assumed to belong to a certain ensemble (e.g., the circular ensemble of Sec. III.C.4).

The Hamiltonian approach in the regime of many overlapping resonances can be traced back to the statistical theory of nuclear reactions (see, for example, Hauser and Feshbach, 1952, and Feshbach, 1992). Ericson fluctuations (Ericson, 1960, 1963; Ericson and Mayer-Kuckuk, 1966) of the cross section of statistical nuclear reactions as a function of energy are a manifestation of the interference of a large number of overlapping resonances. The Hamiltonian approach was followed by Verbaarschot, Weidenmüller, and Zirnbauer (1985) in calculating the autocorrelation function of $S$-matrix elements, and by Fyodorov and Sommers (1996a, 1996b, 1997) in calculating various statistical properties of the $S$ matrix in chaotic systems.

The random- $S$-matrix approach was introduced in the theory of statistical nuclear reactions by Mello, Pereyra, and Seligman (1985) and by Friedman and Mello (1985), through the maximal entropy approach. A similar approach was applied to quantum transport in disordered metals; see, for example, the review by Stone et al. (1991). The connection between the statistics of the eigenphases in chaotic scattering and the circular ensembles was suggested by Blümel and Smilansky (1988, 1989, 1990); for a review see Smilansky (1990). Direct applications of the random- $S$-matrix approach to transport in open dots were initiated by Baranger and Mello (1994) and Jalabert, Pichard, and Beenakker (1994); for reviews see Mello (1995), Beenakker (1997), and Mello and Baranger (1999). Both approaches turn out to be equivalent when the external parameters of the system are fixed, as proven by Brouwer (1995). Parametric correlations can be successfully derived in the Hamiltonian approach but not in the $S$-matrix approach.

In the Hamiltonian approach, we assume a Hamiltonian $H$ that is taken from the appropriate Gaussian ensemble of random matrices. The statistical properties of the $S$ matrix and of the conductance can then be inferred from Eq. (20). Various moments and correlation functions of the $S$ matrix depend only on combinations of the dot-leads coupling coefficients $W_{\mu c}$ that are invariant under the transformations that leave the ensemble distribution invariant. Such invariant combinations are

$$
M_{c c^{\prime}} \equiv \frac{2 \pi}{N} \sum_{\mu} W_{\mu c}^{*} W_{\mu c^{\prime}},
$$

measuring the degree of correlation among the open channels. For example, the average $S$ matrix is given by

$$
\bar{S}=\frac{I-\pi M / 2 \Delta}{I+\pi M / 2 \Delta}
$$

where $M$ is the matrix defined in Eq. (89). It follows from Eq. (90) that the matrix $M=2 \pi W^{\dagger} W / N$ is completely determined by $\bar{S}$, and therefore all moments and correlations of the statistical $S$ matrix are functions of $\bar{S}$ only.

The average $S$ matrix is not unitary. The eigenvalues $T_{c}$ of $1-\bar{S} \bar{S}^{\dagger}$ measure the unitary deficiency of the $S$ matrix $\left(0 \leq T_{c} \leq 1\right)$ and are called the transmission coefficients. In a set of "eigenchannels" for which the $M$ is diagonal $\left(M_{c c^{\prime}}=w_{c}^{2} \delta_{c c^{\prime}}\right), \bar{S}$ is diagonal too and $T_{c} \equiv 1-\left|\bar{S}_{c c}\right|^{2}=\left(2 \pi w_{c}^{2} / \Delta\right) /\left(1+\pi w_{c}^{2} / 2 \Delta\right)^{2}$.

As long as we work at a fixed energy and given values of the external parameters, it is possible to convert the original probability density (58) for the Hamiltonian $H$ to a probability density $P(S)$ in the space of unitary $S$ matrices of the respective symmetry. It was shown by Brouwer (1995) that for $\Lambda_{\text {tot }}=\Lambda_{1}+\Lambda_{2}$ open channels

$$
P(S) \propto\left|\operatorname{det}\left(1-\bar{S}^{\dagger} S\right)\right|^{-\beta\left(\Lambda_{\mathrm{tot}}-1\right)-2},
$$

where $\bar{S}$ is given by Eq. (90) and the measure $d \mu(S)$ is that of the corresponding circular ensemble. For $\bar{S} \neq 0$, Eq. (91) is known as the Poisson kernel (Hua, 1963). It was derived by Pereyra and Mello (1983) in nuclear physics through maximizing the entropy $\mathcal{S}[P(S)]=$ $-\int d \mu(S) P(S) \ln P(S)$ of an arbitrary distribution $P(S)$ subjected to the constraints $\overline{S^{n}}=\bar{S}^{n}$ (for any positive integer $n$ ). For $\bar{S}=0$, we recover Dyson's circular ensemble for the scattering matrices (see Sec. III.C.4). Indeed, owing to the invariance (81) of the circular ensemble's measure, the average $S$ matrix must satisfy $\bar{S}=\overline{U S V}=U \bar{S} V$ for arbitrary unitary matrices $U$ and $V$, a condition that can only be met by $\bar{S}=0$. This corresponds to the case of ideal leads where all $T_{c}=1$.

Thus far we have not distinguished between channels belonging to the left and right leads. This distinction becomes important in calculating the conductance through the dot. In general, the $S$ matrix is defined by the linear relation between the incoming and outgoing amplitudes. It can be written in the form $S=\left(\begin{array}{cc}r & t^{\prime} \\ t & r^{\prime}\end{array}\right)$, where $r$ and $r^{\prime}$ are the reflection matrices on the left and on the right, respectively, while $t$ and $t^{\prime}$ are transmission matrices from left to right and from right to left, respectively. For $\Lambda_{1}$ $\left(\Lambda_{2}\right)$ channels in the left (right) lead, $r$ and $r^{\prime}$ are square matrices of dimension $\Lambda_{1} \times \Lambda_{1}$ and $\Lambda_{2} \times \Lambda_{2}$, while $t$ and $t^{\prime}$ are rectangular matrices of order $\Lambda_{1} \times \Lambda_{2}$ and $\Lambda_{2} \times \Lambda_{1}$.

Here $t t^{\dagger}$ and $t^{\prime} t^{\prime \dagger}$ share the same number $\min \left(\Lambda_{1}, \Lambda_{2}\right)$ of nonzero transmission eigenvalues $\tau_{a}$. The zerotemperature conductance is proportional to the total transmission $T$ :

$$
T=\operatorname{Tr}\left(t t^{\dagger}\right)=\operatorname{Tr}\left(t^{\prime} t^{\prime \dagger}\right)=\sum_{a} \tau_{a} .
$$

For ideal leads (i.e., $\bar{S}=0$ ), the distribution of the transmission eigenvalues is given by (Baranger and Mello, 1994; Jalabert, Pichard, and Beenakker, 1994) 


$$
P_{\beta}\left(\tau_{1}, \tau_{2}, \ldots\right) \propto\left(\prod_{a<b}\left|\tau_{a}-\tau_{b}\right|^{\beta}\right) \prod_{c} \tau_{c}^{\frac{\beta-2}{2}} .
$$

In the more general case of nonideal leads, the Poisson kernel (91) should be taken into account beyond Eq. (93); see Brouwer, 1995.

\section{B. The semiclassical approach}

The semiclassical approach to open dots is useful in the limit of a large number of modes $\Lambda \rightarrow \infty$ (equivalent to $\hbar \rightarrow 0)$. The starting point is an expression for the transmission amplitude $t_{c^{\prime} c}$ from mode $c$ in the left lead to mode $c^{\prime}$ in the right lead in terms of the retarded Green's function $G^{R}$. Assuming the dot-lead interfaces are at $x=x^{l}$ and $x=x^{r}$, we have

$$
\begin{aligned}
t_{c^{\prime} c}= & -i \hbar\left(v_{c^{\prime}} v_{c}\right)^{1 / 2} \int d y^{\prime} d y \phi_{c^{\prime}}^{r *}\left(y^{\prime}\right) \\
& \times G^{R}\left(x^{l}, y ; x^{r}, y^{\prime} ; E\right) \phi_{c}^{l}(y),
\end{aligned}
$$

where $\phi_{c}^{l(r)}(y)=\sqrt{2 / \mathcal{W}} \sin (c \pi y / \mathcal{W})(c=1, \ldots, \Lambda)$ are the transverse channel wave functions in the left (right) lead ( $\mathcal{W}$ is the width of each lead). The retarded Green's function is then approximated by a sum over classical paths [Eq. (49)]. The integrals over the lead-dot cross sections are done by stationary phase, leading to (Jalabert, Baranger, and Stone, 1990)

$$
\begin{aligned}
t_{c^{\prime} c}= & -\left(\frac{\pi i}{2 k \mathcal{W}}\right)^{1 / 2} \sum_{\alpha\left(\bar{c}^{\prime} \bar{c}\right)} \operatorname{sign}\left(\bar{c}^{\prime}\right) \operatorname{sign}(\bar{c}) \\
& \times \tilde{A}_{\alpha} e^{\frac{i}{\hbar} \tilde{S}_{\alpha}\left(\bar{c}^{\prime}, \bar{c}, E\right)-i \frac{\pi}{2} \tilde{\mu}_{\alpha}},
\end{aligned}
$$

where $\bar{c}= \pm c$ and $\tilde{\mu}_{\alpha}$ is a modified Maslov index (given by Baranger, Jalabert and Stone, 1993b). The sum is taken over paths $\alpha$ that start on the left at angle $\theta$ and end on the right at angle $\theta^{\prime}$, determined by equating the initial and final transverse momenta of the electron to the quantized values of the momenta in modes $c$ and $c^{\prime}$, respectively (i.e., $\sin \theta=\pi \bar{c} / k \mathcal{W}$ and $\sin \theta^{\prime}=\pi \bar{c}^{\prime} / k \mathcal{W}$ ). The modified amplitude and action are given by

$$
\begin{aligned}
\tilde{A}_{\alpha}\left(\theta, \theta^{\prime}\right) & =\left[\frac{1}{\mathcal{W}\left|\cos \theta^{\prime}\right|}\left|\left(\frac{\partial y}{\partial \theta^{\prime}}\right)_{\theta}\right|\right]^{1 / 2}, \\
\tilde{S}_{\alpha}\left(\bar{c}^{\prime}, \bar{c}, E\right) & =S_{\alpha}\left(y_{0}^{\prime}, y_{0}, E\right)+\pi \hbar \bar{c} y_{0} / \mathcal{W}-\pi \hbar \bar{c}^{\prime} y_{0}^{\prime} / \mathcal{W},
\end{aligned}
$$

where $y_{0}$ and $y_{0}^{\prime}$ are determined from the stationary phase conditions for the angles $\theta$ and $\theta^{\prime}$. Note that the energy dependence in the sum of Eq. (95) appears only in the action $\tilde{S}_{\alpha}=k \tilde{L}_{\alpha}$ (where $\tilde{L}_{\alpha}$ is the effective length of path $\alpha)$.

Expression (95) holds only for "chaotic" isolated trajectories that scatter from the boundary of the dot before exiting on the right. Direct trajectories give rise to nonuniversal effects, and the geometry of the dot is usually chosen so as to minimize these effects.

\section{Mesoscopic fluctuations of the conductance}

1. Conductance distributions

According to Landauer's formula, the zero-temperature dimensionless conductance $g$ $\left[g=G /\left(2 e^{2} / h\right)\right]$ is the total transmission $T=\sum_{a} \tau_{a}$, and its distribution for ideal leads can be determined from the joint distribution (93) of the transmission eigenvalues. For single-mode ideal leads $\left(\Lambda_{1}=\Lambda_{2}=1\right.$; Baranger and Mello, 1994; Jalabert, Pichard, and Beenakker, 1994),

$$
P(g)=\frac{1}{2} \beta g^{\frac{\beta-2}{2}} \quad(0<g<1) .
$$

The GOE and GUE distributions are shown by solid lines in the upper panels of Fig. 11. In the absence of a magnetic field $(\beta=1)$, it is more probable to find smaller conductances; in the presence of a magnetic field $(\beta=2)$, all allowed values are equally probable; and in the presence of spin-orbit scattering $(\beta=4)$, larger conductances are more probable.
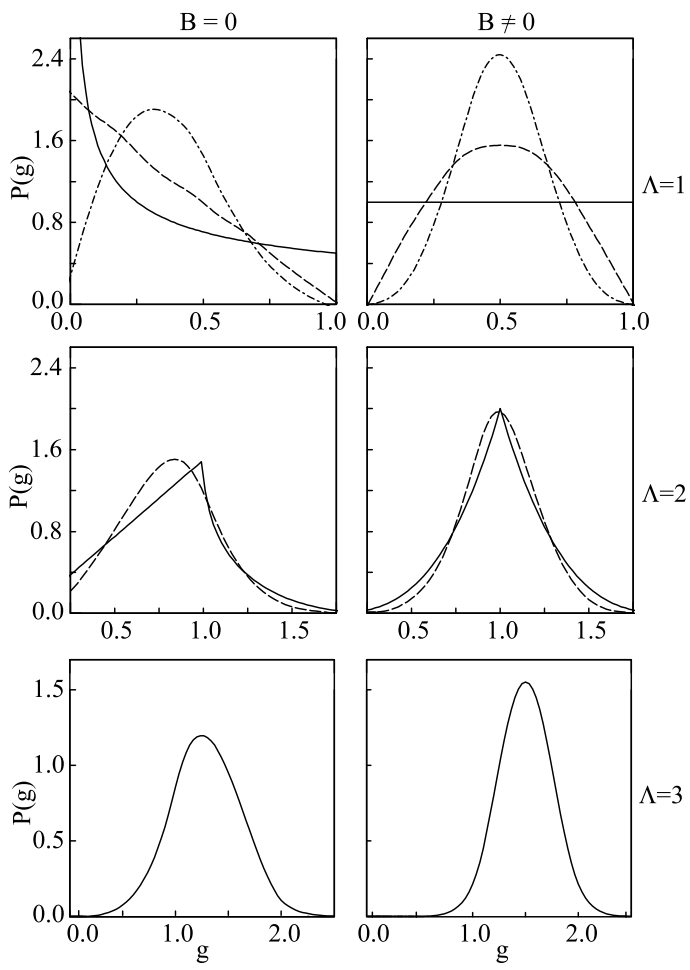

FIG. 11. Conductance distributions in open dots with $\Lambda=$ 1,2 and 3 channels in each lead for conserved (left) and fully broken (right) time-reversal symmetries. The distributions are calculated using Dyson's circular random matrix ensembles: solid lines, the distributions in the absence of phase breaking; dashed lines (shown for $\Lambda=1,2$ only), the results of the voltage-probe model with a single-mode phase-breaking lead $\left(\Lambda_{\phi}=1\right)$; dash-dotted lines (shown for $\Lambda=1$ only), $\Lambda_{\phi}=2$. Adapted from Baranger and Mello (1994, 1995). 
The conductance distributions are also sensitive to the number of channels in each lead and were calculated for $\Lambda_{1}=\Lambda_{2}=\Lambda \leq 3$ (Baranger and Mello, 1994) using the circular ensembles. The results are shown by the solid lines in Fig. 11. Already for $\Lambda=3$ they are quite close to a Gaussian, which is their exact asymptotic limit $(\Lambda \rightarrow \infty)$. The average and variance are given by

$$
\begin{aligned}
\bar{g}= & \frac{\Lambda}{2}-\frac{\Lambda}{2(2 \Lambda-1+2 / \beta)}\left(\frac{2}{\beta}-1\right) \\
& \rightarrow \frac{\Lambda}{2}-\frac{1}{4}\left(\frac{2}{\beta}-1\right), \\
\sigma^{2}(g)= & \frac{2}{\beta} \frac{\Lambda^{2}(\Lambda-1+2 / \beta)^{2}}{(2 \Lambda+2+2 / \beta)(2 \Lambda-1+4 / \beta)(2 \Lambda-1+2 / \beta)^{2}} \\
& \rightarrow \frac{1}{8 \beta},
\end{aligned}
$$

where the limit is $\Lambda \rightarrow \infty$. The asymptotic values were derived earlier by Iida, Weidenmüller, and Zuk (1990a, 1990b) in the Hamiltonian approach.

The dominating term $\Lambda / 2$ in the average conductance (98a) is just its classical value, while the second term is the quantum weak-localization correction (see below). Its asymptotic value is $-1 / 4$ and 0 for conserved and broken time-reversal symmetry, respectively.

One conclusion from Eq. (98b) is that the rms of the conductance fluctuations is of order $e^{2} / h$, irrespective of the size of the average conductance $\Lambda e^{2} / h$ or the size of the system (the number of modes $\Lambda=\operatorname{int}[k \mathcal{W} / \pi]$ is size dependent). This is a manifestation of the phenomenon of universal conductance fluctuations, known from disordered metals. In the limit $\Lambda \rightarrow \infty$, the conductance variance is twice as large in the absence of magnetic field than in its presence.

The case of nonideal leads was treated in the Hamiltonian approach by Iida, Weidenmüller, and Zuk (1990a, 1990b). The $S$-matrix approach (where the appropriate ensemble is the Poisson kernel) was followed by Brouwer and Beenakker (1994). For equivalent channels $\left(T_{c}=T\right)$, and in the limit $\Lambda T \gg 1$, one can integrate over the ensemble by diagrammatic methods (Brouwer and Beenakker, 1996).

Direct paths connecting the leads can also lead to $\bar{S} \neq 0$. This situation is analogous to nuclear reactions in which both direct and equilibrated components contribute to the reaction cross section (Feshbach, 1992). Baranger and Mello (1996) extracted an average "optical" $S$ matrix through an energy average of the numerical data (in cavities). The distributions predicted by the corresponding Poisson kernel (91) were then found to be in good agreement with the direct numerical calculations.

We can gain additional physical insight into the behavior of the average conductance and its weak-localization correction in the semiclassical approximation (Baranger, Jalabert and Stone, 1993a). Using Eq. (95) in the di- agonal approximation (see Sec. III.B.1) and replacing $\sum_{c} \rightarrow \int_{-1}^{1} d \sin \theta$, we obtain for the average transmission $\bar{T}=\Lambda \mathcal{T}$, where $\mathcal{T}$ is the classical transmission probability per incoming mode (Beenakker and van Houten, 1989). This result agrees with the leading-order term of the RMT result (98a) if $\mathcal{T}=1 / 2$. Indeed, for a fully chaotic system and ideal leads, the electron injected into the dot forgets its origin and has equal probability of exiting on the left or on the right.

The quantum correction to the average transmission is of order unity and cannot be calculated directly in the semiclassical approach. Instead we can evaluate such a correction to the average of the total reflection $R=\sum_{c^{\prime} c}\left|r_{c^{\prime} c}\right|^{2}$. To leading order $\bar{R} \approx \Lambda \mathcal{R}$, where $\mathcal{R}$ is the classical reflection probability. However, if time reversal is a good symmetry, a correction to the diagonal part of the reflection $\bar{R}_{D}=\overline{\sum_{c}\left|r_{c c}\right|^{2}}$ comes from pairs of time-reversed orbits - they have the same action and Maslov index, and their contribution to the diagonal reflection does not average to zero. We find that the correction to $\bar{R}_{D}$ is $\delta \bar{R}_{D}=\frac{1}{2} \int_{-1}^{1} d \sin \theta \sum_{\alpha(\theta, \pm \theta)}\left|\tilde{A}_{\alpha}(\theta, \theta)\right|^{2}$, where $A_{\alpha}(\theta, \theta)$ is given by Eq. (96a) for $\theta=\theta^{\prime}$, and the sum is over all classical paths $\alpha$ that enter and exit at angle $\theta$. For chaotic trajectories the function $P\left(\theta, \theta^{\prime}\right) \equiv \sum_{\alpha\left(\theta, \theta^{\prime}\right)}\left|\tilde{A}_{\alpha}\left(\theta, \theta^{\prime}\right)\right|^{2}$ satisfies approximately a uniformity condition $P(\theta, \theta) \approx \int_{-1}^{1} d \sin \theta^{\prime} P\left(\theta, \theta^{\prime}\right)$ (Baranger, Jalabert, and Stone, 1993b), leading to the weak-localization correction (for conserved time-reversal symmetry)

$$
\delta \bar{R}_{D}=\mathcal{R}
$$

In the absence of time-reversal symmetry, the return probability to the same channel is $\overline{\left|r_{c c}\right|^{2}}=$ $\overline{\sum_{c^{\prime}}\left|r_{c^{\prime} c}\right|^{2}} / \Lambda \approx \mathcal{R} / \Lambda$. When time-reversal symmetry is conserved, this probability increases by $\delta \bar{R}_{D} / \Lambda \approx \mathcal{R} / \Lambda$, so its value doubles to $2 \mathcal{R} / \Lambda$.

How does the weak-localization correction to the reflection affect the conductance? We use the unitarity condition of the $S$ matrix $\overline{\left|S_{c c}\right|^{2}}+\sum_{c^{\prime} \neq c} \overline{\left|S_{c^{\prime} c}\right|^{2}}=1$, and assume that all off-diagonal probabilities $\overline{\left|S_{c^{\prime} c}\right|^{2}}\left(c^{\prime} \neq c\right)$ are equal. An increase in the return probability $\overline{\left|r_{c c}\right|^{2}}=$ $\overline{\left|S_{c c}\right|^{2}}$ by $\mathcal{R} / \Lambda$ must then be compensated for by a decrease $\approx \mathcal{R} / 2 \Lambda^{2}$ in each individual off-diagonal probability. The change in the total average transmission $\bar{T}=\sum_{c^{\prime} \in r, c \in l} \overline{\left|S_{c^{\prime} c}\right|^{2}}$ is then $\delta \bar{T} \approx-\mathcal{R} / 2$. For ideal leads, $\mathcal{R}=1 / 2$, and $\delta \bar{T}=-1 / 4$, in agreement with Eq. (98a).

The enhancement of the mean-square diagonal $S$ matrix element relative to the mean-square off-diagonal element due to time-reversal symmetry is well known in the statistical theory of nuclear reactions, where it is called the elastic enhancement factor. It was also observed in quantum chaotic scattering (Doron, Smilansky, and Frenkel, 1990; Blümel and Smilansky, 1992). 


\section{Weak localization}

In Sec. IV.C.1 we saw that the average conductance increases when time-reversal symmetry is fully broken. In this section we discuss the dependence of $\bar{g}$ on a magnetic field $B$ as time-reversal symmetry is broken gradually.

The semiclassical expression for $\bar{g}$ versus $B$ was derived by Baranger, Jalabert, and Stone (1993a). For a weak magnetic field the classical trajectories are approximately the same as for $B=0$, but the wave function acquires an Aharonov-Bohm phase. The contribution to the action in the presence of a field $B$ derived from a vector potential $\boldsymbol{A}$ is $(e / c) \int \boldsymbol{A} \cdot \boldsymbol{d} \boldsymbol{\ell}$. Under time reversal, this action changes sign, and the action difference between paths that are time-reversed pairs is nonzero: $\left(\tilde{S}_{\alpha}-\tilde{S}_{\alpha^{T}}\right) / \hbar=(2 e / \hbar c) \int \boldsymbol{A} \cdot \boldsymbol{d} \boldsymbol{\ell}=2 \Theta_{\alpha} B / \Phi_{0}$, where $\Theta_{\alpha}$ is the area (times $2 \pi$ ) swept by the classical trajectory $\alpha$. Following the method that led to Eq. (99), we now find

$$
\delta \bar{R}_{D}(B)=\mathcal{R} \int d \Theta P(\Theta) e^{2 i \frac{\Theta B}{\Phi_{0}}}
$$

where $P(\Theta) \equiv \frac{1}{2} \int d \sin \theta d \sin \theta^{\prime} \sum_{\alpha\left(\theta, \theta^{\prime}\right)}\left|\tilde{A}_{\alpha}\right|^{2} \delta\left(\Theta-\Theta_{\alpha}\right)$ is the distribution of the effective area $\Theta$ enclosed by the electron trajectory. This area distribution is exponential in chaotic systems (Doron, Smilansky, and Frenkel, 1990; Jalabert, Baranger, and Stone, 1990) but satisfies only a power law in nonchaotic systems. Using an exponential form $P(\Theta) \propto e^{-\alpha_{c l}|\Theta|}$ in Eq. (100), and the unitarity argument discussed at the end of Sec. IV.C.1, we find

$$
\begin{aligned}
\delta g(B) \equiv \bar{g}^{\mathrm{GUE}}-\bar{g}(B) & =\frac{\mathcal{R}}{2} \frac{1}{1+\left(B / B_{c r}\right)^{2}}, \\
B_{c r} & =\alpha_{c l} \Phi_{0} / 2 .
\end{aligned}
$$

Here $B_{c r}$ is the crossover field measuring a typical field required to suppress the weak-localization correction. $\delta g(B)$ is largest at $B=0$ (note that the average conductance itself has a $\operatorname{dip}$ at $B=0$ ).

In a chaotic system $\alpha_{c l} \propto \tau_{\text {escape }}^{-1 / 2}$ (Jensen, 1991). The basic argument is that area accumulation in a chaotic dot is diffusive and $\overline{\Theta^{2}}$ is therefore linear in time. Since the average time spent by the electron in the dot is $\tau_{\text {escape }}$, we have $\left(\overline{\Theta^{2}}\right)^{1 / 2} / \mathcal{A} \propto\left(\tau_{\text {escape }} / \tau_{c}\right)^{1 / 2}(\mathcal{A}$ is the dot's area and $\tau_{c}$ is the ergodic time). From $\alpha_{c l} \propto\left(\overline{\Theta^{2}}\right)^{-1 / 2}$ and using Eq. (101b), we obtain for the crossover flux $\Phi_{c r}=B_{c r} \mathcal{A}$

$$
\frac{\Phi_{c r}}{\Phi_{0}}=\frac{1}{2} \kappa\left(2 \pi \frac{\tau_{c}}{\tau_{\text {escape }}}\right)^{1 / 2}=\frac{1}{2} \kappa\left(2 \pi \frac{\bar{\Gamma}}{E_{T}}\right)^{1 / 2},
$$

where $E_{T}=\hbar / \tau_{c}$ is the ballistic Thouless energy and $\bar{\Gamma}=\hbar / \tau_{\text {escape }}$ is the mean escape width of a level in the dot. The quantity $\kappa$ is a geometrical factor that depends on the device. In a dot with a total of $2 \Lambda$ ideal open channels, $\bar{\Gamma}=(\Delta / 2 \pi) 2 \Lambda$, and $\Phi_{c r} / \Phi_{0}=(\kappa / 2) g_{T}^{-1 / 2} \sqrt{2 \Lambda}$, where $g_{T} \equiv E_{T} / \Delta$ is the ballistic Thouless conductance. In particular, $B_{c r} \propto \sqrt{\Lambda}$.

The exact weak-localization line shape $\delta g(B)$ for a small number of channels was calculated in the Hamiltonian approach using the crossover ensemble (68) for $H$ in Eq. (20) for the $S$ matrix (Pluhar et al., 1994). The exact expression (evaluated by supersymmetry) is a complicated triple integral [see, for example, Eq. (6) of Pluhar et al., 1994], but it is well approximated by a Lorentzian

$$
\delta g(B) \approx \delta g(0) \frac{1}{1+\left(\zeta / \zeta_{c r}\right)^{2}},
$$

where $\delta g(0)=\Lambda /(4 \Lambda+2)$, in agreement with the circular ensemble result (98a), and $\zeta_{c r}=$ $(\sqrt{\Lambda} / 2 \pi)(1-1 / 2 \Lambda)^{-1 / 2}$. This crossover scale of the conductance is different from the crossover scale of the spectral statistics $(\sim 1)$, and can be understood in terms of a competition between the mixing time $\hbar / \tau_{\text {mix }}=2 \pi \Delta \zeta^{2}$ and the decay time of a typical resonance in the dot $\hbar / \tau_{\text {escape }}=(\Delta / 2 \pi) \Lambda$. The conductance statistics make the crossover when $\tau_{\text {escape }} / \tau_{\text {mix }}=4 \pi^{2} \zeta^{2} / \Lambda \sim 1$ or $\zeta_{c r} \sim \sqrt{\Lambda} / 2 \pi$.

Comparing the RMT result [Eq. (103)] with the semiclassical result [Eq. (101)], we conclude that if the timereversal symmetry is broken by a magnetic field, then $\zeta \propto B$. This result is confirmed in billiard-model calculations (Bohigas et al., 1995; Alhassid, Hormuzdiar, and Whelan, 1998). A semiclassical expression can be derived for the proportionality constant (Bohigas et al., 1995; Pluhar̆ et al., 1995). Obviously $\zeta_{c r}$ and $B_{c r}$ satisfy the same proportionality relation, confirming our earlier conclusion that $B_{c r} \propto \sqrt{\Lambda}$.

We have seen in Sec. IV.C.1 that the conductance variance in open dots is reduced by a factor of 2 when time-reversal symmetry is broken. Using the transition random-matrix ensemble (68), Frahm (1995) derived the complete dependence of the conductance variance on the transition parameter $\zeta$. In the limit $\Lambda \gg 1$,

$$
\sigma_{\zeta}^{2}(g)-\sigma_{\mathrm{GUE}}^{2}(g) \approx\left[\frac{\delta \sigma^{2}(g)}{1+\left(\zeta / \zeta_{c r}\right)^{2}}\right]^{2}
$$

where $\sigma_{\mathrm{GUE}}^{2}=1 / 16$ and $\delta \sigma^{2}(g) \equiv \sigma_{\mathrm{GOE}}^{2}(g)-\sigma_{\mathrm{GUE}}^{2}(g)=$ $1 / 16$.

The weak-localization effect has been observed in open quantum dots by several experimental groups (Marcus et al., 1992; Berry et al., 1994a, 1994b; Chang et al., 1994; Keller et al., 1994, 1996; Chan et al., 1995). Methods employed to find the average conductance include averaging over energy (Berry et al., 1994a, 1994b; Keller et al., 1996), over shapes (Chan et al., 1995), and over an ensemble of dots (Chang et al., 1994).

Figure 12 shows $-[\bar{G}(B)-\bar{G}(0)]=\delta G(B)-\delta G(0)$ as a function of magnetic field $B$ for two different ballistic cavities - a stadium [panels (a) and (c)] and a circle 
[panels (b) and (d)]. Panels (a) and (b) display the experimental result of Chang et al. (1994) using an array of $6 \times 8$ "identical" quantum dots. The ensemble average shows a clear weak-localization effect. While the line shape for the stadium is a Lorentzian [Eq. (101)], expected for a chaotic cavity, the line shape for the circle is triangular, consistent with a power-law area distribution that is expected for an integrable system. Figures 12(c) and $12(\mathrm{~d})$ show the results of numerical calculations for the same 2D geometries used in the experiment.
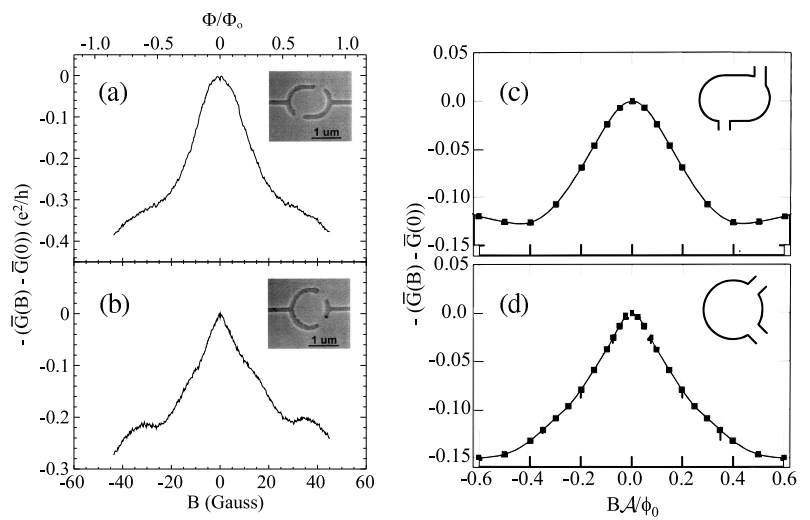

FIG. 12. Weak-localization effect in open quantum dots: experiment [panels (a) and (b)] vs theory [panels (c) and (d)]. The experimental results for $-[\bar{G}(B)-\bar{G}(0)]$ (averaged over 48 dots) are shown for (a) a stadium and (b) a circle. The observed line shape is a Lorentzian for the stadium (a chaotic structure) and triangular for the circle (a regular system). The insets are electron micrographs of the fabricated microcavities. Numerical results of the weak-localization effect are shown for (c) a stadium and (d) a circle. The circle's cavity includes a weak disorder potential to account for possible impurities in the experimental structures. Note the similarity between experiment and theory for both geometries. Adapted from Chang et al. (1994).

Chan et al. (1995) fabricated a dot whose shape could be distorted and collected statistics at each value of the magnetic field $B$ from various shapes of the dot. The measured weak-localization effect and the variances of the conductance fluctuations (for $\Lambda=2$ channels in each lead) are well fitted to Eqs. (103) and (104), respectively $\left(\zeta / \zeta_{c r}=B / B_{c r}=2 B / \alpha \Phi_{0}\right.$ was used in these formulas). However, the parameters found in the fit to the data $\delta g=0.15$ (where $g$ is the conductance in units of $\left.2 e^{2} / h\right)$ and $\delta \sigma^{2}(g)=0.00475$ are significantly smaller than the RMT values of $\delta g=0.2$ and $\delta \sigma^{2}(g) \approx 0.0229$ for $\Lambda=2$ [see Eqs. (98)]. This can be explained by dephasing effects that will be discussed in Sec. IV.E. The characteristic inverse area parameter $\alpha$ is found to be $\alpha=0.14 \pm 0.01 \mu \mathrm{m}^{-2}$ and $\alpha=0.11 \pm 0.01 \mu \mathrm{m}^{-2}$ from fits to the weak-localization line shape (103) and variance curve (104), respectively. An independent measurement of this parameter is from the power spectrum of the conductance fluctuations versus magnetic field (see Sec. IV.C.4).

\section{Ericson fluctuations}

One of the prominent features of stochastic nuclear reactions is the rapid fluctuation of the cross section $\sigma$ versus the total reaction energy $E$ [see, for example, Fig. $6(a)]$. Ericson $(1960,1963)$ quantified these fluctuations in terms of the energy autocorrelation function

$$
c_{\sigma}(\Delta E)=\overline{\sigma(E+\Delta E) \sigma(E)}-\overline{\sigma(E)}^{2} \propto \frac{\Gamma^{2}}{(\Delta E)^{2}+\Gamma^{2}},
$$

where the average is taken over E. According to Ericson, this autocorrelation function is a Lorentzian whose width measures the average resonance width $\Gamma$ in the compound nucleus.

Do Ericson fluctuations also occur in quantum dots? The experimental results in Fig. 6(c), showing the conductance as a function of the Fermi momentum, exhibit fluctuations that are indeed similar to those observed in nuclear reactions. The quantity analogous to Eq. (105) is the conductance autocorrelation function versus the Fermi momentum $k: c_{g}(\Delta k) \equiv \overline{g(k+\Delta k) g(k)}-\bar{g}^{2}$. This correlator can be estimated semiclassically (Jalabert, Baranger, and Stone, 1990). Similar work for the autocorrelations of the $S$-matrix elements in chaotic systems was done by Blümel and Smilansky (1988, 1989, 1990). Using the Landauer formula and the semiclassical expression (95) for the transmission amplitudes, we find

$$
c_{g}(\Delta k)=\left|\int_{0}^{\infty} d L e^{i \Delta k L} P(L)\right|^{2},
$$

where $P(L) \equiv \frac{1}{2} \int d \sin \theta d \sin \theta^{\prime} \sum_{\alpha\left(\theta, \theta^{\prime}\right)}\left|\tilde{A}_{\alpha}\right|^{2} \delta\left(L-\tilde{L}_{\alpha}\right)$ is the classical distribution of path lengths (the sum is over all trajectories that originate and end at the same angles $\theta$ and $\left.\theta^{\prime}\right)$. In a chaotic system the length distribution is exponential: $P(L) \propto e^{-\gamma_{c l} L}$ and $c_{g}(\Delta k)$ is a Lorentzian,

$$
c_{g}(\Delta k) \propto \frac{1}{1+\left(\Delta k / \gamma_{c l}\right)^{2}} .
$$

Thus the scale for the conductance variation as a function of the Fermi momentum is set by $\gamma_{c l}$, the inverse average length of a chaotic trajectory traversing the dot. To relate Eq. (107) to Ericson's formula (105), it is necessary to convert $\gamma_{c l}$ to a correlation length in energy $E_{c o r}=\hbar v_{F} \gamma_{c l}$. Using $\gamma_{c l}=1 / v_{F} \tau_{\text {escape }}$, we find $E_{\text {cor }} \approx \hbar / \tau_{\text {escape }}=\bar{\Gamma}$, in agreement with Ericson's formula.

Fluctuations of the conductance as a function of the Fermi momentum were studied experimentally by 
Keller et al. (1996). A typical measurement of $G$ versus $k_{F}$ is shown in Fig. 6(c). While the average conductance increases linearly with the number of modes $k \mathcal{W} / \pi$, the fluctuations are of universal size $\sigma^{2}(g) \sim 1$. The power spectrum $S_{g}\left(f_{k}\right)$ of $g(k)\left[S_{g}\left(f_{k}\right)=\left|\int d k e^{2 \pi i f_{k}} g(k)\right|^{2}\right]$ is the Fourier transform of the conductance autocorrelator $c_{g}(\Delta k)$, and for a Lorentzian line shape [Eq. (107)] we expect an exponential power spectrum $S_{g}\left(f_{k}\right)=$ $S_{g}(0) e^{-2 \pi \gamma_{c l}\left|f_{k}\right|}$ [see, for example, Fig. 6(d)]. Numerical calculations give good agreement between the classical value of $\gamma_{c l}$ and its value extracted from fitting an exponential to the quantum calculations of the power spectrum (Jalabert, Baranger, and Stone, 1990).

The autocorrelation function $c_{g}^{\zeta}(\Delta E)$ for a fixed value of the transition parameter $\zeta$ in the crossover regime between GOE and GUE was calculated in RMT for ideal leads with $\Lambda \gg 1$ (Frahm, 1995):

$c_{g}^{\zeta}(\Delta E)=\frac{1}{16}\left\{\frac{1}{\left[1+\left(\frac{\zeta}{\zeta_{c r}}\right)^{2}\right]^{2}+\left(\frac{\Delta E}{\Gamma}\right)^{2}}+\frac{1}{1+\left(\frac{\Delta E}{\Gamma}\right)^{2}}\right\}$.

Equation (108) reduces to a Lorentzian in both the GOE $(\zeta=0)$ and $\operatorname{GUE}(\zeta \gg 1)$ limits, in agreement with the semiclassical result (107).

\section{Parametric correlations}

In this section we discuss the mesoscopic fluctuations of the conductance as a function of an external parameter. Note that the energy should not be considered an external parameter since it enters in a special way in Eq. (20) for the $S$ matrix, while a dependence on a generic external parameter $x$ is introduced through $H=H(x)$.

Of particular experimental interest is the parametric correlator when the parameter varied is a magnetic field: $c_{g}(\Delta B) \equiv \overline{g(B+\Delta B) g(B)}-\bar{g}^{2}$. Following the semiclassical approach of Sec. IV.C.3, we now obtain

$$
c_{g}(\Delta B)=\left|\int_{-\infty}^{\infty} d \Theta e^{i \Theta \Delta B / \Phi_{0}} P(\Theta)\right|^{2},
$$

where $P(\Theta)$ is the classical area distribution swept by the electron in the dot. In a chaotic dot, $P(\Theta) \propto e^{-\alpha_{c l}|\Theta|}$ (see Sec. IV.C.2), and $c_{g}(\Delta B)$ is a squared Lorentzian:

$$
c_{g}(\Delta B) \propto\left[1+\left(\Delta B / 2 B_{c r}\right)^{2}\right]^{-2} .
$$

The correlation field $B_{c}$ is thus twice the crossover field $B_{c r}$ [Eq. (101b)], because the phase involved in the correlator is proportional to the difference of areas as opposed to their sum in the weak localization case.
Typical conductance fluctuations versus magnetic field and the conductance correlator $c_{g}(\Delta B)$ in an open stadium dot are shown in Fig. 13(a) and (b), respectively (Jalabert, Baranger, and Stone, 1990). The calculated correlator [solid line in panel (b)] compares well with the semiclassical prediction (110) [dashed line in panel (b)]. The power spectrum of $g(B)\left[S_{g}\left(f_{B}\right) \equiv\right.$ $\left.\overline{\left|\int d B e^{i 2 f_{B} B} g(B)\right|^{2}}\right]$ is just the Fourier transform of $c_{g}(\Delta B)$ and is given by (Marcus et al., 1993b)

$$
S_{g}\left(f_{B}\right)=S_{g}(0)\left(1+2 \pi \alpha \Phi_{0}\left|f_{B}\right|\right) e^{-2 \pi \alpha \Phi_{0}\left|f_{B}\right|},
$$

where we have used $B_{c r}=\alpha \Phi_{0} / 2$ [see Eq. (101b)]. The inset of Fig. 13(b) shows the calculated power spectrum $S_{g}\left(f_{B}\right)$ (solid line) in comparison with a best fit to Eq. (111) (dashed line). Good agreement between the classical value of $\alpha$ (determined from the area distribution in the stadium) and the quantum value of $\alpha$ [determined from a fit of (111) to the power spectrum] is found over a variety of the stadium dot's parameters.
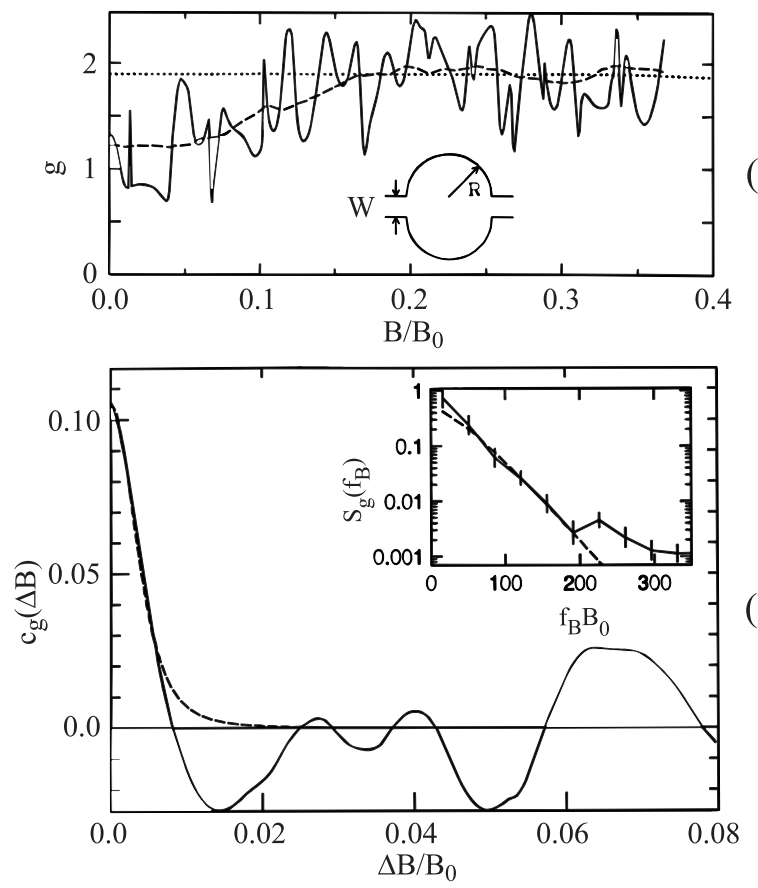

FIG. 13. Conductance fluctuations vs magnetic field in an open chaotic dot: (a) dimensionless conductance $g$ vs $B / B_{0}$ (where $B_{0}=m c v_{F} / e \mathcal{W}$ ) for an open stadium with $R / \mathcal{W}=2$ (see inset) and $k_{F} \mathcal{W} / \pi=4.5$; solid line, the quantum calculation; dashed line, the smoothed quantum result; dotted line, the classical result. (b) The calculated conductance correlator $c_{g}(\Delta B)$ vs $\Delta B / B_{0}$ for the open stadium (solid line) and its semiclassical prediction (110) (dashed line). The inset shows the smoothed power spectrum $S_{g}\left(f_{B}\right)$ of $g(B)$ [i.e., the Fourier transform of $\left.c_{g}(\Delta B)\right]$. The dashed line is the best fit to Eq. (111). From Jalabert, Baranger and Stone (1990).

The power spectrum $S_{g}\left(f_{B}\right)$ was determined in the experiment of Chan et al. (1995) by averaging over indi- 
vidual spectra from the measurement of $g(B)$ at different shapes. A two-parameter $\left[S_{g}(0)\right.$ and $\left.\alpha\right]$ fit to (111) reproduces the data well with $\alpha=0.14 \pm \mu \mathrm{m}^{-2}$, in agreement with the value derived from the weak-localization effect (see Sec. IV.C.2). In the same experiment the conductance $g$ was also measured versus a shape-distortion voltage $V$. Figure $6(\mathrm{~d})$ shows the power spectrum $S_{g}\left(f_{V}\right)$, averaged at ten different measurements of $g(V)$ collected at various fixed values of the magnetic field. The dotted line is a fit to $S_{g}(V)=S_{g}(0) e^{-2 \pi \xi f_{V}}$, suggesting a Lorentzian line shape for the conductance correlator versus shape: $c_{g}(\Delta V) \propto 1 /\left[1+(\Delta V / \xi)^{2}\right]$.

Using RMT, it is possible to calculate the correlation function in both energy and a time-reversal-symmetrybreaking parameter $\zeta$. For ideal leads with $\Lambda \gg 1$ and for completely broken time-reversal symmetry (Frahm, 1995),

$$
c_{g}(\Delta \zeta, \Delta E)=\frac{1}{16} \frac{1}{\left[1+\left(\Delta \zeta / 2 \zeta_{c r}\right)^{2}\right]^{2}+(\Delta E / \Gamma)^{2}} .
$$

For $\Delta E=0$, Eq. (112) reduces to the squared Lorentzian of Eq. (110). Efetov (1995) calculated this correlator for nonideal leads. Asymptotic expressions for the conductance correlator $c_{g}(\Delta \zeta)$ in RMT were derived for any number of channels $\Lambda$ by Gossiaux, Pluhar̆, and Weidenmüller (1998) and by Pluhar̆ and Weidenmüller (1999). The limits considered were small and large $\Delta \zeta$ at fixed $\Lambda$, as well as large $\Lambda$ at fixed $\Delta \zeta / \Lambda$.

\section{Conductance fluctuations at finite temperature}

Temperature affects the conductance fluctuations in two ways, through thermal smearing and through the increase of the dephasing rate with temperature. Both effects reduce the fluctuations. In this section we discuss the first effect, i.e., the role of temperature in mesoscopic fluctuations in the limit of complete phase coherence.

Efetov (1995) derived the conductance correlator versus magnetic field $c_{g}(\Delta B ; T)$ at finite temperature $T$. Using Eq. (11) for the finite-temperature conductance, this correlator can be related to the zerotemperature correlator $c_{g}(\Delta B, \Delta E)$ in both magnetic field and energy (see Sec. IV.C.4): $c_{g}(\Delta B ; T)=$ $T^{2} \int_{-\infty}^{\infty} \frac{d}{d T}[2 T \sinh (\Delta E / 2 T)]^{-2} c_{g}(\Delta B, \Delta E) d \Delta E$. At low temperatures $T \ll \Gamma / 2 \pi$, this correlator reduces to the squared Lorentzian of Eq. (110), while at high temperatures $T \gg \Gamma / 2 \pi$, this correlator is a Lorentzian,

$$
c_{g}(\Delta B ; T)=\frac{\pi \Gamma}{96 T} \frac{1}{1+\left(\Delta B / 2 B_{c r}\right)^{2}} .
$$

The high-temperature limit of the conductance variance $\sigma^{2}(g)=\pi \Gamma / 96 T$ can be seen to be much smaller than the zero-temperature variance of $1 / 16$. Thus the conductance fluctuations are reduced significantly when the temperature exceeds a typical level width $\Gamma$.

\section{E. Dephasing}

The magnitudes of the observed weak-localization correction and conductance fluctuations in open dots are often reduced compared to the theoretical expectations. This discrepancy is explained by dephasing times that are comparable with typical escape times in the dot. Dephasing can be caused by a voltage probe (since an electron absorbed by the probe is reinjected into the dot with an uncorrelated phase) or by inelastic processes in the dot such as electron-electron collisions.

There are two important issues concerning dephasing. The first is to describe quantitatively how mesoscopic fluctuations are affected by finite dephasing rates (Sec. IV.E.1). The second is the dependence of the dephasing rate on temperature (Sec. IV.E.2). Recent experimental results in dots with single-mode leads are described in Sec. IV.E.3.

\section{Models for dephasing}

The dephasing voltage-probe model was introduced by Büttiker (1986a, 1986b) and applied to the conductance fluctuations in open dots by Baranger and Mello (1995) and by Brouwer and Beenakker (1995). A third dephasing lead is added and its voltage $V_{\phi}$ is adjusted to keep $I_{\phi}=0$, thus conserving the average number of electrons in the dot. The effective two-lead conductance $2\left(e^{2} / h\right) g=I_{1} /\left(V_{2}-V_{1}\right)$ can be expressed in terms of the three probe conductance coefficients:

$$
g=g_{21}+\frac{g_{2 \phi} g_{\phi 1}}{g_{\phi 2}+g_{\phi 1}} .
$$

Each of the first two leads has $\Lambda$ equivalent modes, and the third lead is assumed to have $\Lambda_{\phi}$ modes. The dephasing rate $\Gamma_{\phi}$ is related to the total number $\Lambda_{\phi}$ of dephasing modes by

$$
\Lambda_{\phi}^{\mathrm{eff}} \equiv \Lambda_{\phi} T_{\phi}=2 \pi \Gamma_{\phi} / \Delta,
$$

where $T_{\phi}$ is the transmission probability per mode in the third lead. The parameter $\Lambda_{\phi}^{\text {eff }} \equiv \Lambda_{\phi} T_{\phi}$ measures the effective number of ideal dephasing modes.

The entire $\left(2 \Lambda+\Lambda_{\phi}\right) \times\left(2 \Lambda+\Lambda_{\phi}\right) S$ matrix is assumed to be described by the respective circular ensemble. The fluctuations of the measured conductance $g$ are then calculated from Eq. (114). Using the relations $g_{21}+g_{22}+g_{2 \phi}=0, g_{11}+g_{21}+g_{\phi 1}=0$ and $g_{12}+g_{22}+g_{\phi 2}=0$ (see Sec. II.B), it is possible to eliminate from Eq. (114) all conductance coefficients that are 
related to the third lead. The distribution of $g$ can then be inferred from the known distribution of the sub- $S$ matrix (Pereyra and Mello, 1983; Friedman and Mello, 1985) that corresponds to the two physical leads (Brouwer and Beenakker, 1995).

Figure 11 shows conductance distributions calculated by Baranger and Mello (1995) for $\Lambda_{\phi}=1$ dephasing mode in open dots with single- or double-mode leads (dashed lines) and for $\Lambda_{\phi}=2$ in dots with single-mode leads (dashed-dotted lines). They also calculated analytically (to leading order in $1 / \Lambda_{\phi}$ ) the average and variance of the conductance for an ideal, multimode voltage probe,

$$
\begin{gathered}
\bar{g}=\frac{\Lambda}{2}+\left(\frac{\Lambda}{\Lambda_{\phi}}\right) \frac{\beta-2}{2 \beta}+\mathcal{O}\left(\Lambda_{\phi}^{-2}\right) \\
\sigma^{2}(g)=\left(\frac{\Lambda}{\Lambda_{\phi}}\right)^{2} \frac{2 \Lambda+2-\beta}{4 \beta \Lambda}+\mathcal{O}\left(\Lambda_{\phi}^{-3}\right),
\end{gathered}
$$

and found an interpolation formula for the weaklocalization correction (at $B=0$ ):

$$
\delta g \equiv-\left(\bar{g}(B=0)-\frac{\Lambda}{2}\right) \approx-\frac{1}{4} \frac{1}{1+\Lambda_{\phi} / 2 \Lambda} .
$$

We see from Eq. (117) that the universal asymptotic result for the weak-localization correction $(-1 / 4)$ is valid only if $\Lambda_{\phi} \ll 2 \Lambda$.

The voltage-probe model describes localized dephasing (at the point contact between the dot and the third lead) and is less suitable to describe dephasing due to inelastic processes that occur through the whole dot. A way to introduce dephasing uniformly over the dot is to add an imaginary part $-i \Gamma_{\phi} / 2$ to the dot's Hamiltonian. This model does not conserve the number of electrons and was modified by Brouwer and Beenakker (1997) to conserve electron number by mapping it on the voltageprobe model. Compared with the standard voltage-probe model, they found conductance distributions that were narrower and with strongly suppressed tails. This is because the number-conserving imaginary-potential model is more effective in dephasing than the localized ideal voltage-probe model. Nevertheless, the asymptotic results for the average and variance coincide exactly with Eqs. (116). Fig. 14(b) shows the dephasing rate $\Gamma_{\phi}$ [calculated from $\Lambda_{\phi}^{\mathrm{eff}}$ via Eq. (115)] versus the weaklocalization correction $\delta g$ for both dephasing models.

The complete form of the weak-localization line shape was calculated in the (non-number-conserving) imaginary-potential model (Efetov, 1995): $\delta g(B) \equiv$ $\bar{g}^{\mathrm{GUE}}-\bar{g}(B)=(1 / 4)\left[1+\left(B / B_{c r}\right)^{2}+\Lambda_{\phi}^{\mathrm{eff}} / 2 \Lambda\right]^{-1}$, and found to have no explicit dependence on temperature. This temperature independence is used to determine the dephasing rate from the measured weak-localization effect at finite temperature, as is discussed in the next section.

\section{Temperature dependence of dephasing}

Dephasing rates were determined experimentally in disordered 2D (Choi, Tsui, and Alavi, 1987) and 1D (Kurdak et al., 1992) semiconductors, in disordered 1D metals (Lin and Giordano, 1986; Echternach, Gersheson and Bozler, 1993), and more recently in open quantum dots (Marcus et al., 1993a, 1994; Clarke et al., 1995; Huibers, Switkes, Marcus, Campman, and Gossard, 1998). To determine $\tau_{\phi}$ experimentally, it is best to measure a quantity that is sensitive to dephasing, yet is not affected explicitly by thermal smearing, e.g., the weak-localization effect in the magnetoconductance. This was recently implemented by Huibers, Switkes, Marcus, Campman, and Gossard (1998) in open quantum dots using three independent methods:

(i) Measuring the weak-localization correction $\delta g$ (at $B=0$ ). From $\delta g$ one can determine the number of effective dephasing channels $\Lambda_{\phi}^{\mathrm{eff}}$ using one of the phenomenological dephasing models [e.g., Eq. (117)] and therefore determine $\Gamma_{\phi}$ from Eq. (115). The inset in Fig. 14(b) shows $\Gamma_{\phi}$ versus $\delta g$ in the two dephasing models discussed in Sec. IV.E.1.

Figure 14 shows the recent measurements of Huibers, Switkes, Marcus, Campman, and Gossard (1998). Panel (a) is the measured $\delta g$ for four different dots as a function of temperature. The smaller values of $\delta g$ for larger dots are consistent with Eq. (115) since $\Delta \propto \mathcal{A}^{-1}$ and $\Lambda_{\phi}^{\text {eff }} \propto$ $\mathcal{A}$ for a fixed value of the dephasing rate (i.e., at fixed temperature). The extracted values of $\tau_{\phi}$, shown in Fig. 14(b) (symbols), are approximately independent of the dot's area.

(ii) Measuring the width of the weak-localization line shape. According to Eqs. (101) or (103), $\delta g(B)$ is a Lorentzian characterized by a crossover field $B_{c r}$ that is proportional to the square root of the total number of open channels. In the presence of phase breaking, this total number of channels should include the number of dephasing channels

$$
B_{c r} \mathcal{A} / \Phi_{0}=(\kappa / 2) g_{T}^{-1 / 2} \sqrt{2 \Lambda+\Lambda_{\phi}^{\mathrm{eff}}},
$$

where $\kappa$ is a geometrical constant of the dot and $g_{T}$ is the Thouless conductance. Equation (118) can be used to determine $\Lambda_{\phi}^{\text {eff }}$ from the measured $B_{c r}$. This equation contains an additional unknown parameter $\kappa$, but it is temperature independent and can be determined, for example, by a best fit to the $\delta g$ data.

(iii) Measuring the power spectrum $S_{g}\left(f_{B}\right)$ of the conductance fluctuations versus magnetic field (Marcus et al., 1993a, 1994; Clarke et al., 1995). At higher temperatures, the conductance correlator in open dots is given by a Lorentzian (113) and its Fourier transform is exponential: $S_{g}\left(f_{B}\right)=S_{g}(0) e^{-2 \pi i B_{c}\left|f_{B}\right|}\left(\right.$ where $\left.B_{c}=2 B_{c r}\right)$. Using a two-parameter fit to the measured power spectrum it is possible to extract the correlation field $B_{c}$ and 
then use Eq. (118) to determine the dephasing time, as in the second method above.

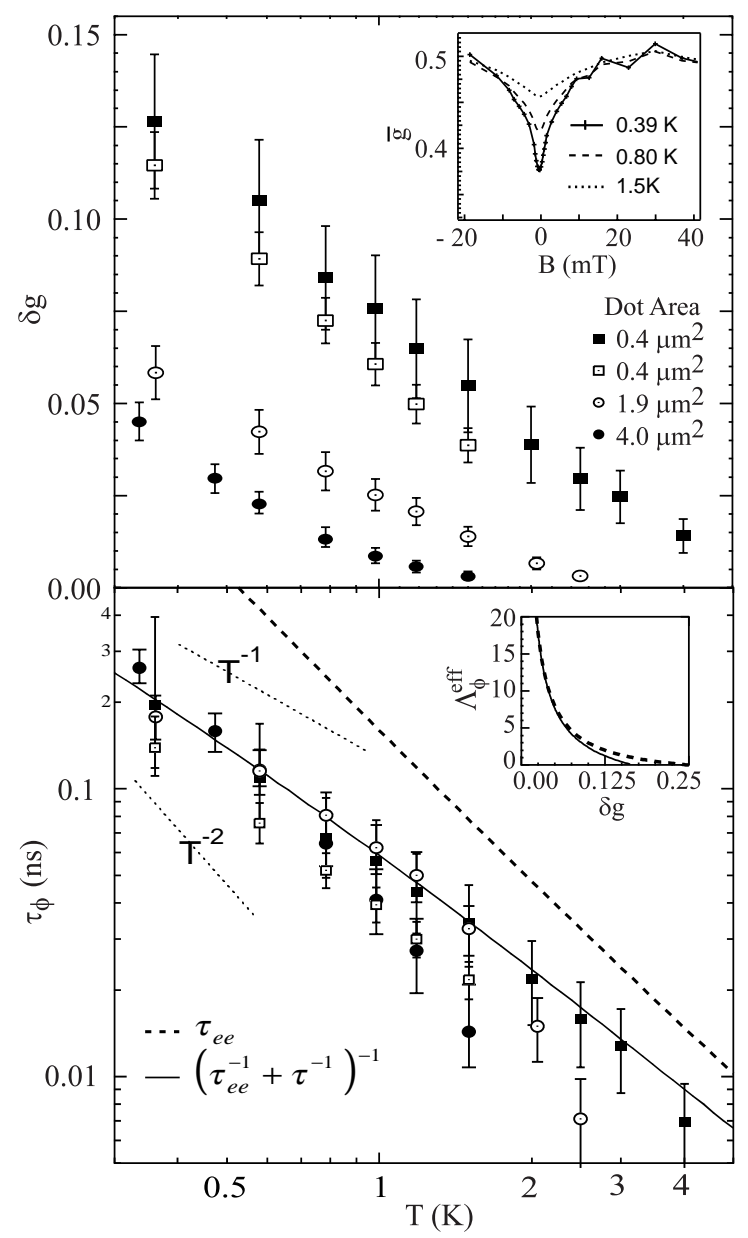

(a)

(b)

FIG. 14. The measured temperature dependence of the dephasing time in open quantum dots: (a) measured weaklocalization correction $\delta g$ in four dots as a function of temperature $T$; note the decrease in $\delta g$ for the larger dots [in agreement with Eq. (115), see text]; inset, $\bar{g}$ vs magnetic field at several temperatures for the first device; (b) symbols, the extracted dephasing time vs temperature; dashed line, $\tau_{e e}$ from Eq. (117); solid line, a fit to the theoretical $\tau_{\phi}=\left(\tau_{e e}^{-1}+\tau^{-1}\right)^{-1}$ of a $2 \mathrm{D}$ disordered system (see text) with $l=0.25 \mu \mathrm{m}$; inset, the effective number of dephasing channels $\Lambda_{\phi}^{\text {eff }}$ vs $\delta g$ in the voltage-probe model (dashed line) and in the number-conserving imaginary-potential model (solid line). Adapted from Huibers, Switkes, Marcus, Campman, and Gossard (1998).

The dependence of the dephasing time on temperature in disordered conductors is theoretically understood (Altshuler and Aronov, 1985; for a recent review see Aleiner, Altshuler, and Gershenson, 1999). At low temperatures, the electron-electron scattering rate dominates the electron-phonon rate. There are two contributions to the electron-electron dephasing rate in 2D: a large-energy- transfer contribution quadratic in $T$ that is characteristic of clean metals, $\tau_{e e}^{-1}=\left[(\pi k T)^{2} / 2 h E_{F}\right] \ln \left(E_{F} / k T\right)$ (see, for example, Pines and Nozières, 1966), and a smallenergy-transfer $(\lesssim k T)$ contribution (Nyquist rate) linear in $T, \tau^{-1}=\left(k T \lambda_{F} / h l\right) \ln \left(\pi l / \lambda_{F}\right)$ (Altshuler and Aronov, 1985; Imry, 1996). The total rate is approximately $\tau_{\phi}^{-1} \approx \tau^{-1}+\tau_{e e}^{-1}$. At low temperatures the Nyquist rate dominates, and it vanishes as $T \rightarrow 0$. Some experiments (e.g., Mohanty, Jariwala, and Webb, 1997) find an apparent saturation of the dephasing rate as $T \rightarrow 0$ for a reason that is not yet understood.

In ballistic quantum dots (0D systems) the situation is less clear. Microscopic estimates in closed ballistic dots by Sivan et al. (1994) and Altshuler et al. (1997) give $\tau_{\phi}^{-1} \propto T^{2}$ for $k T \gg \Delta$. However, no theoretical estimates are available for open quantum dots. The results of Huibers, Switkes, Marcus, Campman, and Gossard (1998) offer a puzzle: they suggest a temperature dependence of the rate that is characteristic of $2 \mathrm{D}$ disordered systems and not just a $T^{2}$ dependence [see Fig. 14(b)]. More experiments and theoretical work may be necessary to resolve this issue.

\section{Conductance distributions}

Using the experimentally determined dephasing rates, one can apply the phase-breaking models described in Sec. IV.E.1 to predict the conductance distributions and compare them with the experimental distributions. Realistic calculations should include effects due both to dephasing and to thermal smearing.

A detailed comparison between experiment and theory for single-mode leads was reported by Huibers, Switkes, Marcus, Brouwer, et al. (1998). The measured weaklocalization correction was used to determine $\tau_{\phi}(T)$ as in Sec. IV.E.2, and found to be consistent with the results shown in Fig. 14(b). The number-conserving imaginary-potential model (Sec. IV.E.1) was then used together with a thermal smearing procedure to calculate the conductance distributions. Figure 15 compares some of these theoretical distributions with the measured ones for both conserved (left) and fully broken (right) timereversal symmetry. The distributions measured at the lower temperature [panels (a) and (c)] are clearly asymmetric. The dotted lines in panels (a) and (c) of Fig. 15 are the predicted RMT distributions (97) without any phase breaking and at $T=0$. The dashed lines include the correct dephasing rate at the corresponding temperature but ignore thermal smearing effects. Finally, the solid lines include both dephasing and explicit thermal effects and are in good agreement with the data. The results show that both direct thermal effects and finite dephasing rates play an important role. 


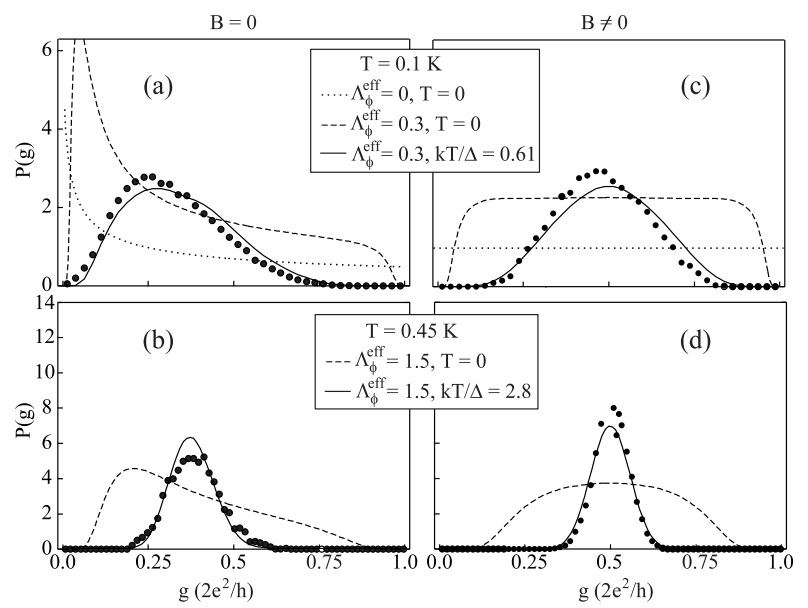

FIG. 15. Finite-temperature conductance distributions in single-mode open dots without a magnetic field [panels (a) and (b)] and with a magnetic field [panels (c) and (d)].Panels (a) and (c) are for $k T=0.61 \Delta$ and panels (b) and (d) are for $k T=2.8 \Delta$. The solid circles are the experimental results. The dotted lines in panels (a) and (c) are the fully phase-coherent $T=0$ distributions (97). The dashed lines in all panels are the theoretical calculations without thermal smearing but with the experimentally determined effective number of dephasing modes at the relevant temperatures $\Lambda_{\phi}^{\mathrm{eff}}=0.3$ in panels (a) and (c), and $\Lambda_{\phi}^{\mathrm{eff}}=1.5$ in panels (b) and (d). The solid lines in all panels are the theoretical predictions when both the finite dephasing rate and thermal smearing effects are accounted for. Adapted from Huibers, Switkes, Marcus, Brouwer, et al. (1998).

\section{MESOSCOPIC FLUCTUATIONS IN CLOSED DOTS}

Recent studies of statistical fluctuations in quantum dots have focused on almost-isolated or "closed" dots that are separated by barriers from the leads. Our main interest is in the quantum Coulomb-blockade regime, where the temperature is comparable to or smaller than the mean level spacing in the dot. Experimentally, a series of peaks is observed in the conductance versus gate voltage; see, for example, Fig. 7(c). The width of these peaks is thermally broadened, but their heights fluctuate strongly. Whereas in open dots the conductance fluctuations originate from the interference of many overlapping resonances, in closed dots the peak-height fluctuations result from the spatial fluctuations of individual resonance wave functions at the dot-lead interfaces.

A statistical theory of the conductance peaks in Coulomb-blockade dots was introduced by Jalabert, Stone and Alhassid (1992). The peak-height distributions were derived from RMT, and found to be universal and sensitive only to the symmetry class. The sensitivity of a Coulomb-blockade peak to an experimentally controlled parameter can be studied by changing a magnetic field or the shape of the dot. Alhassid and Attias (1996a) derived the peak-height parametric correlation function and showed that it is universal once the parameter is scaled. The predicted statistics of the peak heights for both conserved and broken time-reversal symmetry were observed by two experimental groups (Chang et al., 1996; Folk et al., 1996) using dots of different design and size. The latter group also confirmed the predicted functional form of the peak-height autocorrelation function in a magnetic field.

In a closed dot, the charge on the dot is quantized and the Coulomb interactions cannot be ignored. The simplest model is the constant-interaction model (26), which takes into account only the average Coulomb energy of the dot's electrons. Interaction effects beyond the constant-interaction model are probably less relevant for the fluctuations of sufficiently highly excited states. However, interactions may cause deviations from RMT for the low-lying part of the spectrum, which is the region of interest in this section. We shall see that the constantinteraction model can explain some, but not all, of the observed statistical fluctuations. The main evidence for the breaking of the constant-interaction model comes from the peak-spacing fluctuations. In the constantinteraction model, these fluctuations reflect the spacings between the single-particle levels in the dot and are expected to follow a Wigner-Dyson distribution. However, the observed distribution is more symmetric and closer to a Gaussian (Sivan et al., 1996; Simmel, Heinzel and Wharam, 1997; Patel, Cronenwett, et al., 1998). A discussion of interaction effects beyond the constantinteraction model is deferred to Sec. VI.

Charging energy affects the statistics of the Coulombblockade peaks at finite temperature. An outstanding issue has been the observed peak-to-peak correlations: temperature enhances these correlations but the experimental correlations at low temperatures are stronger than expected. An interesting phenomenon is the saturation of peak-to-peak correlations as a function of temperature (Patel, Stewart, et al., 1998). This effect can be explained by the statistical scrambling of the dot's spectrum as electrons are added to the dot.

Section V.A describes the main elements of the statistical theory of closed dots. Sections V.B and V.C review the statistics and parametric correlations respectively, of the conductance peaks. The crossover statistics in the presence of a time-reversal symmetry-breaking field are discussed in Sec. V.D. The peak-spacing statistics in the framework of a single-particle model plus constant charging energy are reviewed in Sec. V.E. Finally, a statistical theory at finite temperature, and the use of temperature to probe the statistical scrambling of the dot's spectrum versus electron number, are presented in Secs. V.F and V.G, respectively. 


\section{A. Statistical theory at low temperatures}

We first discuss Coulomb-blockade dots at $T \ll \Delta$. Typically these low temperatures are still much larger than a typical resonance width and the observed conductance peaks are thermally broadened (see Sec. II.D). The interesting information is carried by the conductance peak height $G_{\lambda}^{\text {peak }} \propto \Gamma^{l} \Gamma^{r} /\left(\Gamma^{l}+\Gamma^{r}\right)$ [see Eq. (24)], where $\Gamma^{l(r)}$ is the width of a resonance level to decay in to the left (right) lead. Here $\Gamma^{l(r)}=\sum_{c}\left|\gamma_{c}^{l(r)}\right|^{2}$, where $\gamma_{c}^{l(r)}$ is the partial amplitude to decay into channel $c$ in the left (right) lead. The quantity $\gamma_{c \lambda}$ is given by Eq. (17), and can be expressed as a scalar product of the resonance wave function $\boldsymbol{\psi}_{\lambda}=\left(\psi_{\lambda 1}, \psi_{\lambda 2}, \ldots\right)$ and the channel wave function $\phi_{c}=\left(\phi_{c 1}, \phi_{c 2}, \ldots\right)$ :

$$
\gamma_{c \lambda}=\left\langle\phi_{c} \mid \boldsymbol{\psi}_{\lambda}\right\rangle \equiv \sum_{j} \phi_{c j}^{*} \psi_{\lambda j}
$$

Here we expanded the wave function $\Psi_{\lambda}=\sum_{j} \psi_{\lambda j} \rho_{j}$ in a fixed basis $\rho_{j}$ in the dot and defined the channel vector $\phi_{c j} \equiv\left(\hbar^{2} k_{c} P_{c} / m\right)^{1 / 2} \int_{\mathcal{C}} d l \rho_{j}^{*}(\boldsymbol{r}) \phi_{c}(\boldsymbol{r})=\sqrt{2 \pi} W_{j c}[W$ is the dot-lead coupling matrix introduced in Eq. (19)]. The scalar product in Eq. (119) is defined over the dotlead interface and differs from the usual scalar product in the Hilbert space of the dot.

Another modeling of a quantum dot assumes pointlike contacts, and each lead is composed of several such point contacts $\boldsymbol{r}_{c}$ (Prigodin, Efetov, and Iida, 1993; Mucciolo, Prigodin, and Altshuler, 1995). Each point contact constitutes one channel, and the partial width of a level $\lambda$ to decay into it is $\gamma_{c \lambda}=\left(\alpha_{c} \mathcal{A} \Delta / \pi\right)^{1 / 2} \Psi_{\lambda}\left(\boldsymbol{r}_{c}\right)$, where $\alpha_{c}$ is a dot-lead coupling parameter and $\mathcal{A}$ is the area of the dot. The partial width can still be expressed as a scalar product [Eq. (119] but now the channel vector is defined by $\phi_{c j} \equiv\left(\alpha_{c} \mathcal{A} \Delta / \pi\right)^{1 / 2} \rho_{j}^{*}\left(\boldsymbol{r}_{c}\right)$.

Fluctuations in $G_{\lambda}^{\text {peak }}$ arise from fluctuations of the widths $\Gamma^{l}$ and $\Gamma^{r}$. These widths are determined by the partial width amplitudes $\gamma_{c \lambda}$, which in turn are expressed by Eq. (17). The penetration factor $P_{c}$ in Eq. (17) is a smooth function of the Fermi energy (or gate voltage), and fluctuations can only arise from the overlap integral $\int_{\mathcal{C}} d l \phi_{c}^{*} \Psi_{\lambda}$, i.e., from the spatial fluctuations of $\Psi_{\lambda}(\boldsymbol{r})$ across the dot-lead interface $\mathcal{C}$. For a chaotic ballistic dot, these fluctuations are described by RMT. A key relation for connecting the physical quantity (i.e., width) to RMT is Eq. (119), expressing the partial-width amplitude as a projection of the random-matrix eigenfunction $\boldsymbol{\psi}_{\lambda}$ on a fixed channel vector $\phi_{c}$.

\section{B. Conductance peak statistics}

The main goal of this section is to derive the statistical distributions of the conductance peak heights. These distributions were derived by Jalabert, Stone, and Alhassid (1992) using RMT and by Prigodin, Efetov, and Iida (1993) using supersymmetry. The case of correlated channels in two-channel leads was treated by Mucciolo, Prigodin and Altshuler (1995) for broken time-reversal symmetry, and the general case of multimode leads with possibly inequivalent and correlated channels was discussed by Alhassid and Lewenkopf (1995).

\section{Partial-width amplitude distribution}

The joint distribution of the partial-width amplitudes $\gamma=\left(\gamma_{1}, \gamma_{2}, \ldots, \gamma_{\Lambda}\right)$ of a resonance $\lambda$ can be computed from the RMT wave-function statistics. Using Eqs. (66) and (119),

$$
\begin{aligned}
P(\boldsymbol{\gamma})= & \frac{\Gamma(\beta N / 2)}{\pi^{\beta N / 2}} \int D[\boldsymbol{\psi}]\left[\prod_{c=1}^{\Lambda} \delta\left(\gamma_{c}-\left\langle\boldsymbol{\phi}_{c} \mid \boldsymbol{\psi}\right\rangle\right)\right] \\
& \times \delta\left(\sum_{\mu=1}^{N}\left|\psi_{\mu}\right|^{2}-1\right),
\end{aligned}
$$

where $D[\boldsymbol{\psi}] \equiv \prod_{\mu=1}^{N} d \psi_{\mu}$ for the GOE and $D[\boldsymbol{\psi}] \equiv$ $\prod_{\mu=1}^{N} d \psi_{\mu}^{*} d \psi_{\mu} / 2 \pi i$ for the GUE. The integral (120) can be evaluated following the methods of Ullah (1967) and transforming to a new set of orthonormal channels. For $\Lambda \ll N$ and $N \rightarrow \infty$, we recover a Gaussian distribution (Alhassid and Lewenkopf, 1995),

$$
P(\gamma)=(\operatorname{det} M)^{-\beta / 2} \mathrm{e}^{-\frac{\beta}{2} \gamma^{\dagger} M^{-1} \gamma}
$$

The distributions (121) are normalized with the measure $D[\gamma] \equiv \prod_{c=1}^{\Lambda}\left(d \gamma_{c} / \sqrt{2 \pi}\right)$ for the GOE and $D[\gamma] \equiv$ $\prod_{c=1}^{\Lambda}\left(d \gamma_{c}^{*} d \gamma_{c} / 2 \pi i\right)$ for the GUE. They can also be derived (Krieger and Porter, 1963; Ullah, 1963) from the requirement that their form be invariant under orthogonal (unitary) transformations for the GOE (GUE). The quantity $M$ in Eq. (121) is the channel correlation matrix

$$
M_{c c^{\prime}}=\overline{\gamma_{c}^{*} \gamma_{c^{\prime}}}=\frac{1}{N}\left\langle\phi_{c} \mid \phi_{c^{\prime}}\right\rangle
$$

and is identical with the matrix $M=2 \pi W^{\dagger} W / N$ defined in Sec. IV.A [see Eq. (89)]. The eigenvalues $w_{c}^{2}$ of $M$ are just the average partial widths $\bar{\Gamma}_{c}$ [see Eq. (122)], and for the present case of isolated resonances $\bar{\Gamma}_{c} \approx(\Delta / 2 \pi) T_{c}$. For a general set of channels, the matrix $M$ can be nondiagonal (describing correlated channels) and have different diagonal elements (corresponding to inequivalent channels).

We note that in chaotic systems the joint distribution of an eigenfunction's amplitudes at $\Lambda$ spatial points $\boldsymbol{r}_{1}, \ldots, \boldsymbol{r}_{\Lambda}$ is a special case of Eq. (121). Indeed, the partial-width amplitudes of the point-contact model are 
proportional to the wave-function amplitudes at a set of fixed spatial points (see also Srednicki, 1996).

In the point-contact model, $M_{c^{\prime} c}$ is a measure of the spatial wave-function correlations at two different spatial points $\overline{\Psi^{*}(\boldsymbol{r}) \Psi\left(\boldsymbol{r}^{\prime}\right)}$. Expanding the eigenfunction in the fixed basis $\Psi(\boldsymbol{r})=\sum_{j} \psi_{j} \rho_{j}(\boldsymbol{r})$, and using the RMT relation $\overline{\psi_{j}^{*} \psi_{j^{\prime}}}=\delta_{j j^{\prime}} / N$, we find $\overline{\Psi^{*}(\boldsymbol{r}) \Psi\left(\boldsymbol{r}^{\prime}\right)}=$ $\sum_{j} \rho_{j}^{*}(\boldsymbol{r}) \rho_{j}\left(\boldsymbol{r}^{\prime}\right) / N$. The fixed basis $\rho_{j}(\boldsymbol{r})$ is chosen such that the eigenfunction's components are distributed randomly on the unit sphere in $N$ dimensions. Randommatrix theory is expected to describe fluctuations in a chaotic system on a local energy scale. Therefore, for the problem of a free particle in a cavity, we choose this basis to be the free-particle states at the given energy $E=\hbar^{2} k^{2} / 2 m$. In polar coordinates $r, \theta$ such a basis is $\rho_{j}(\boldsymbol{r}) \propto J_{j}(k r) e^{i j \theta}(j=0, \pm 1, \pm 2 \ldots)$, where $J_{j}$ are Bessel functions of the first kind. Using the addition theorem for the Bessel functions, we obtain

$$
\overline{\Psi^{*}(\boldsymbol{r}) \Psi\left(\boldsymbol{r}^{\prime}\right)}=\mathcal{A}^{-1} J_{0}\left(k\left|\boldsymbol{r}-\boldsymbol{r}^{\prime}\right|\right) .
$$

Similar results are obtained if $\rho_{j}$ are chosen to be plane waves with fixed energy but random orientation of momentum $\boldsymbol{k}$. Equation (123) was first derived by Berry (1977), assuming that the Wigner function of an ergodic system is microcanonical on the energy surface and averaging over a spatial region whose linear extension is large compared with the particle's wavelength. It follows that in the point-contact model, the channel correlation matrix of a chaotic dot is given by $M_{c c^{\prime}}=$ $\left(\sqrt{\alpha_{c} \alpha_{c^{\prime}}} \Delta / \pi\right) J_{0}\left(k\left|\boldsymbol{r}_{c}-\boldsymbol{r}_{c^{\prime}}\right|\right)$.

In $d$ dimensions the spatial correlations of eigenfunctions are

$$
\begin{aligned}
\overline{\Psi^{*}(\boldsymbol{r}) \Psi\left(\boldsymbol{r}^{\prime}\right)} / \overline{|\Psi(\boldsymbol{r})|^{2}} & =2^{d / 2-1} \Gamma(d / 2) \frac{J_{d / 2-1}\left(k\left|\boldsymbol{r}-\boldsymbol{r}^{\prime}\right|\right)}{\left(k\left|\boldsymbol{r}-\boldsymbol{r}^{\prime}\right|\right)^{d / 2-1}} \\
& \equiv f_{d}(|\Delta \boldsymbol{r}|)
\end{aligned}
$$

The envelope of $f_{d}(|\Delta \boldsymbol{r}|)$ decays as a power law $(k|\Delta \boldsymbol{r}|)^{-(d-1) / 2}$. For weakly disordered systems, $f_{d}$ contains an additional factor of $e^{-|\Delta \boldsymbol{r}| / 2 l}$, resulting in an exponential cutoff of the spatial correlations beyond $l$ (Mucciolo, Prigodin, and Altshuler, 1995; Prigodin, 1995).

\section{Width distribution}

We next determine the level-width distribution (note that for a symmetric $\operatorname{dot} \Gamma^{l}=\Gamma^{r}$, and the conductance peak height is proportional to the width). Using $\Gamma=\sum_{c}\left|\gamma_{c}\right|^{2}=\gamma^{\dagger} \gamma$ and the Gaussian nature (121) of the partial width amplitudes, we can easily calculate the characteristic function of the width distribution $P(u) \equiv$ $\int_{0}^{\infty} d \Gamma \exp (i u \Gamma) P(\Gamma)=[\operatorname{det}(I-2 i M u / \beta)]^{-\beta / 2}$. This width distribution $P(\Gamma)$ is then (Alhassid and Lewenkopf, 1995)

$$
P(\Gamma)=\frac{1}{2 \pi} \int_{-\infty}^{\infty} d u \frac{e^{-i u \Gamma}}{[\operatorname{det}(I-2 i u M / \beta)]^{\beta / 2}}
$$

and depends only on the eigenvalues $w_{c}^{2}$ of the positivedefinite correlation matrix $M$.

Equation (125) can be evaluated by contour integration. All the singularities of the integrand are along the negative imaginary axis $u=-i \tau$ at $\tau=1 / w_{c}^{2}$. For the GOE case the singularities are of the type $\left(\tau-1 / 2 w_{c}^{2}\right)^{-1 / 2}$, leading to

$$
\begin{aligned}
& P_{G O E}(\Gamma)=\frac{1}{\pi 2^{\Lambda / 2}}\left(\prod_{c} \frac{1}{w_{c}}\right) \sum_{m=1}^{\Lambda}(-)^{m+1} \int_{1 / 2 w_{2 m-1}^{2}}^{1 / 2 w_{2 m}^{2}} d \tau \\
& \times \frac{\mathrm{e}^{-\Gamma \tau}}{\sqrt{\prod_{r=1}^{2 m-1}\left(\tau-\frac{1}{2 w_{r}^{2}}\right) \prod_{s=2 m}^{\Lambda}\left(\frac{1}{2 w_{s}^{2}}-\tau\right)}}
\end{aligned}
$$

where the eigenvalues of $M$ are arranged in ascending order and we have defined $1 / 2 w_{\Lambda+1}^{2} \rightarrow \infty$ for an odd number of channels. For the GUE statistics, all the singularities are poles. If the eigenvalues of $M$ are nondegenerate, then the poles are simple and

$$
\begin{aligned}
P_{G U E}(\Gamma)=\left(\prod_{c} \frac{1}{w_{c}^{2}}\right) \sum_{c=1}^{\Lambda} & {\left[\prod_{c^{\prime} \neq c}\left(\frac{1}{w_{c^{\prime}}^{2}}-\frac{1}{w_{c}^{2}}\right)\right]^{-1} } \\
& \times \mathrm{e}^{-\Gamma / w_{c}^{2}}
\end{aligned}
$$

In the special case of uncorrelated and equivalent channels, all the eigenvalues of $M$ are degenerate, $w_{c}^{2}=w^{2}$, and the width distribution can be found directly from Eq. (125) to be the $\chi^{2}$ distribution in $\beta \Lambda$ degrees of freedom.

\section{Peak-height distributions}

In a closed dot we define $g$ to be the (dimensionless) conductance peak height $G^{\text {peak }}$ measured in units of $\left(e^{2} / h\right)(\pi \Gamma / 4 k T)$ [see Eqs. (24)]. Assuming that the widths $\Gamma^{l}$ and $\Gamma^{r}$ are uncorrelated, $P(g)$ can be computed using Eq. (24b) and the known width distributions of Sec. V.B.2. In the simple case of one-channel symmetric leads $\left(\Lambda=1 ; \bar{\Gamma}^{l}=\bar{\Gamma}^{r}\right)$, we find (Jalabert, Stone, and Alhassid, 1992; Prigodin, Efetov, and Iida, 1993)

$$
\begin{aligned}
& P_{G O E}(g)=\sqrt{2 / \pi g} e^{-2 g}, \\
& P_{G U E}(g)=4 g e^{-2 g}\left[K_{0}(2 g)+K_{1}(2 g)\right],
\end{aligned}
$$

where $K_{0}$ and $K_{1}$ are the modified Bessel functions.

The peak-height distributions were measured independently by Chang et al. (1996) and by Folk et al. (1996) for dots with single-channel tunneling leads. The results of Chang et al. [(1996), Figs. 16(a) and 16(b)] are from dots with $\sim 100$ electrons. The histograms are the experimental results at $T=75 \mathrm{mK} ; 72$ peaks were collected 
for $B=0$ and 216 peaks for $B \neq 0$. The solid lines are the predicted theoretical distributions (128a) and (128b) for $B=0$ and $B \neq 0$, respectively. The conversion from the measured conductance peak $G^{\text {peak }}$ to the dimensionless conductance $g$ requires an unknown parameter - the average width $\bar{\Gamma}$ of a resonance in the dot. Since $\bar{\Gamma}$ is independent of the magnetic field, the theoretical distributions in Figs. 16(a) and 16(b) represent a one-parameter fit $\bar{\Gamma} \approx 0.086 k T$ to both curves. The measured distributions are non-Gaussian and characterized by a large number of small peaks with more small peaks for the $B=0$ case, in agreement with the theoretical predictions. The inset of Fig. 16(a) shows a $B=0$ peak sequence versus gate voltage at $T=75 \mathrm{mK}$ (lower trace); three of the peaks are too small to be observed, but can be seen at a higher temperature trace $(T=600 \mathrm{mK})$ of the same peaks (upper trace).

Figures 16(c) and 16(d) show the experimental results of Folk et al. (1996) at $T=70 \pm 20 \mathrm{mK}$. Their dots are larger $(\sim 1000$ electrons) than in the previous experiment, so that $k T / \Delta \sim 0.3-0.5 \Delta$ is higher. Using shape-distorting gates, larger statistics could be collected. Each distribution includes $\sim 600$ peaks, although only $\sim 90$ are statistically independent. The solid lines are fits to the RMT predictions, (128a) and (128b). The insets show the same distributions on a linear-log scale, where good agreement between theory and experiment is seen over two to three orders of magnitude. We remark, however, that the strong correlations observed between heights of neighboring peaks [see, for example, Fig. 7(c)] is at variance with RMT, and we shall return to this point in Sec. V.F.1.

It is also possible to calculate the peak-height distributions for a general leads configuration for both the orthogonal (Alhassid and Lewenkopf, 1995, 1997) and unitary ( Alhassid and Lewenkopf, 1995, 1997; Mucciolo, Prigodin and Altshuler, 1995) symmetries. The RMT predictions for the peak-height distributions were tested in a model of a ballistic dot - the conformal billiard (see Sec. III.A.3) - in its chaotic regime. Good agreement is found for dots with single-channel (Stone and Bruus, 1993; Bruus and Stone, 1994) and multimode leads (Alhassid and Lewenkopf, 1995, 1997).

The Coulomb-blockade peaks exhibit a weak-localization effect. The average conductance peak height for symmetric leads with $\Lambda$ channels in each lead is

$$
\bar{g}_{\Lambda}=\left\{\begin{array}{cc}
\frac{\Lambda^{2}}{2(\Lambda+1)} & (\mathrm{GOE}) \\
\frac{\Lambda^{2}}{2 \Lambda+1} & (\mathrm{GUE})
\end{array} .\right.
$$

Thus $\bar{g}_{\Lambda}$ for GOE is smaller by an amount $\Lambda^{2} /[2(\Lambda+$ 1) $(2 \Lambda+1)]$ than its GUE value - a weak-localization effect. The relative reduction of the average conductance $\delta g_{\Lambda} / \bar{g}_{\Lambda}^{\mathrm{GUE}}=1 /(2 \Lambda+2)\left(\right.$ where $\left.\delta g_{\Lambda}=\bar{g}_{\Lambda}^{\mathrm{GUE}}-\bar{g}_{\Lambda}^{\mathrm{GOE}}\right)$ is

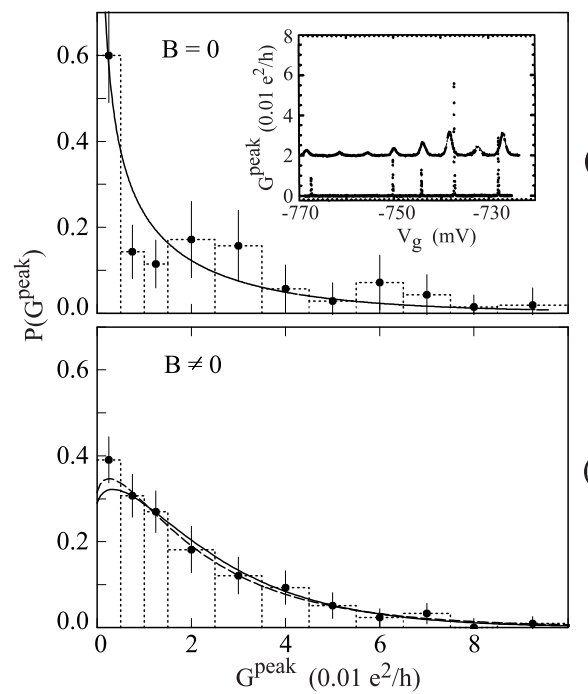

(a)

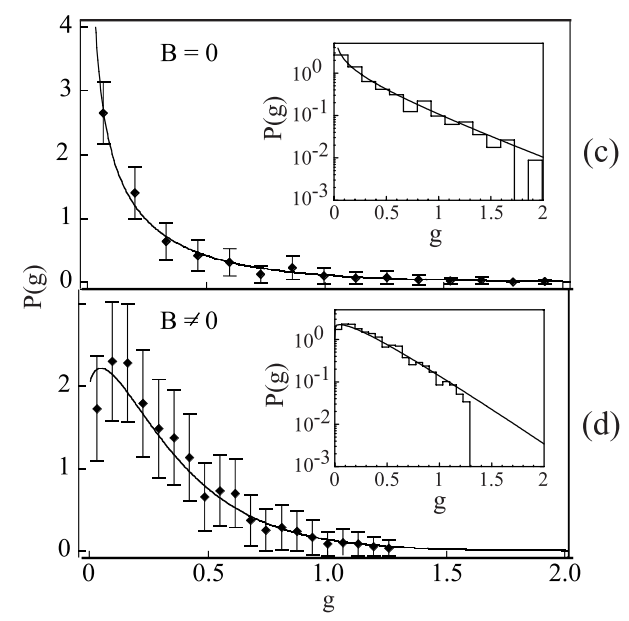

FIG. 16. Conductance peak-height distributions in small and large Coulomb-blockade quantum dots. Panels (a) and (b): measured distributions of Chang et al. (1996) [panel (a)] at $B=0$ and [panel (b)] at $B \neq 0$ in small dots of effective size $0.25 \mu \mathrm{m} \times 0.25 \mu \mathrm{m}$ at $T=75 \mathrm{~K}(\mathcal{N} \sim 100$ electrons and $k T / \Delta \sim 0.15)$; solid lines, the theoretical predictions (128a) and (128b) of Jalabert, Stone, and Alhassid (1992) for conserved and broken time-reversal symmetry, respectively. Inset in panel (a): lower trace, a sequence of $B=0$ Coulombblockade peaks as a function of gate voltage at $T=75 \mathrm{mK}$; upper trace, the same peak series but at $T=660 \mathrm{mK}$. Notice the large fluctuations in the peak heights and the presence of very small peaks (three of them are unobserved) at low temperature. From Chang et al. (1996). Panels (c) and (d): measured peak-height distributions of Folk et al. (1996) in Coulomb-blockade dots at $T=70 \pm 20 \mathrm{mK}$, compared with the theoretical predictions (solid lines) for [panel (c)] $B=0$ and [panel (d)] $B \neq 0$. The two dots used in the experiment, with areas $0.32 \mu \mathrm{m}^{2}$ and $0.47 \mu \mathrm{m}^{2}$, are relatively large $(\mathcal{N} \sim$ 1000). This allows for a larger number of peaks $(\sim 40)$ to be observed in each sweep of the gate voltage, but $k T / \Delta$ is only $\sim 0.3-0.5$. The insets show the same distributions on a loglinear scale (histograms are from the experiment). Adapted from Folk et al. (1996). 
smaller by a factor of $(2 \Lambda+1) /(2 \Lambda+2)$ than its corresponding value for open dots [see Eq. (98a)]. For example, in single-channel leads the weak-localization correction in closed dots is $\delta g / \bar{g}^{\mathrm{GUE}}=1 / 4$ compared with $1 / 3$ in open dots.

In the limit of large $\Lambda$, the GUE variance of the conductance peak heights is smaller by a factor of two than the GOE variance (Alhassid, 1998). A similar behavior was found in open dots [see Eq. (98b)]. For single-channel symmetric leads, the variance of the conductance peak reduces from $1 / 8$ in the GOE to $4 / 45$ in the GUE.

\section{Parametric correlations of the conductance peaks}

In closed dots one can follow a specific conductance peak as a function of an external parameter such as magnetic field or shape [see, for example, Fig. 17(a)], and calculate the correlation between peak heights at different values of the external parameter. Alhassid and Attias (1996a) calculated this conductance peak correlator using the framework of Gaussian processes discussed in Sec. III.C.3 and found it to be universal upon an appropriate scaling of the external parameter.

The parametric width correlator $c_{\Gamma}\left(x-x^{\prime}\right)=$ $\overline{\delta \Gamma(x) \delta \Gamma\left(x^{\prime}\right)} /\left\{\overline{[\delta \Gamma(x)]^{2}} \overline{\left[\delta \Gamma\left(x^{\prime}\right)\right]^{2}}\right\}^{1 / 2}$, where $\delta \Gamma(x) \equiv$ $\Gamma(x)-\bar{\Gamma}(x)$ can be calculated in the framework of the Gaussian process and is universal upon the scaling (77) of the external parameter. It can also be shown to be independent of the channel correlation matrix $M$ (Alhassid and Attias, 1996b) and is therefore determined by the symmetry class alone. The width correlator coincides with the overlap correlator (79) of Sec. III.C.3 in the limit $N \rightarrow \infty$ and is thus well approximated by Eq. (80), a Lorentzian in the Gaussian orthogonal process and a squared Lorentzian in the Gaussian unitary process.

The conductance peak-height correlator

$$
c_{g}\left(x-x^{\prime}\right)=\overline{\delta G(x) \delta G\left(x^{\prime}\right)} /\left\{\overline{[\delta G(x)]^{2}} \overline{\left[\delta G\left(x^{\prime}\right)\right]^{2}}\right\}^{1 / 2}
$$

depends on the number of channels in each lead $\Lambda^{l}$ and $\Lambda^{r}$ and on the eigenvalues $\left(w_{c}^{l, r}\right)^{2}$ of the correlation matrices $M^{l}$ and $M^{r}$ in the left and right leads, respectively. The case most relevant to experiments is that of singlechannel leads. When the leads are symmetric $\left(\bar{\Gamma}^{l}=\bar{\Gamma}^{r}\right)$, the deviation of the corresponding $c_{g}(\Delta x)$ from the width correlator is the largest. This correlator is well fitted by the form of Eq. (80), i.e., a Lorentzian for the Gaussian orthogonal process and a squared Lorentzian for the Gaussian unitary process [see inset in Fig. 17(b)], but with $\alpha_{1}=0.37 \pm 0.04$ and $\alpha_{2}=0.54 \pm 0.04$, respectively.
The universality of the conductance correlator was verified in Anderson-model simulations (Alhassid and Attias, 1996a) as well as in billiard-model calculations (Bruus, Lewenkopf, and Mucciolo, 1996). For multichannel symmetric leads it was found that the conductance correlator approaches the width correlator as the number of channels increases (Alhassid and Attias, 1996b).
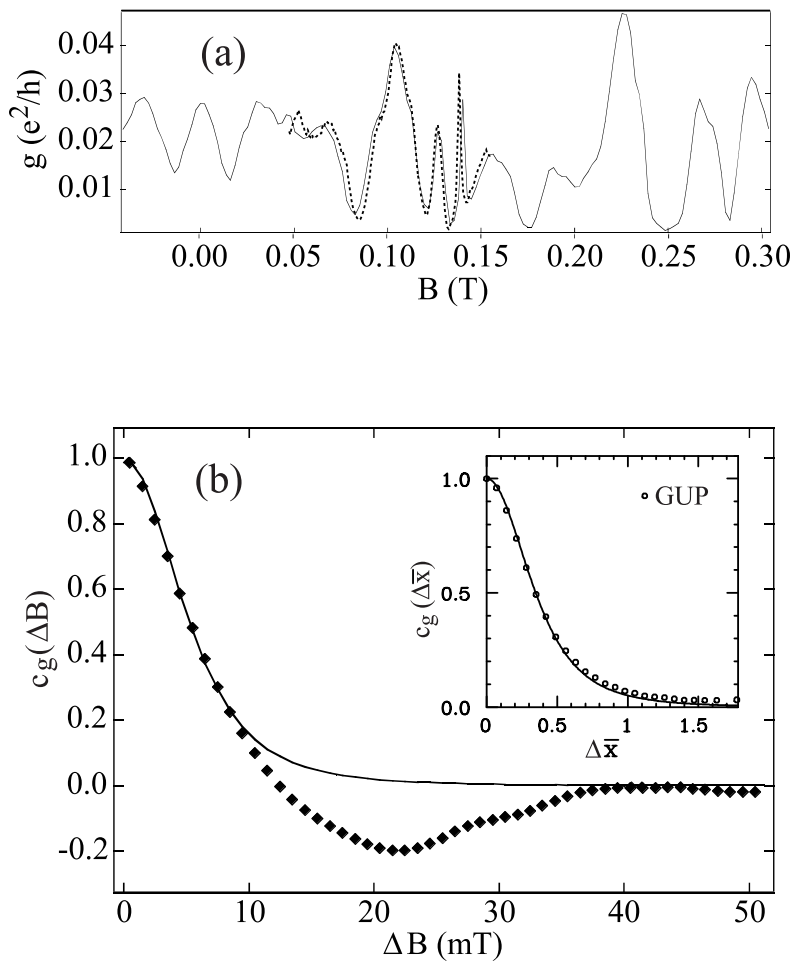

FIG. 17. Conductance peak-height fluctuations vs magnetic field in closed dots: (a) the measured peak height of a single Coulomb-blockade peak as a function of magnetic field $B$ (solid line) and $-B$ (dashed line), showing the symmetry and reproducibility of the data; (b) the peak-height autocorrelation function in a magnetic field $c_{g}(\Delta B)$; diamonds, the experimentally determined peak-height autocorrelation function in a magnetic field $c_{g}(\Delta B)$ at $T=70 \pm 20 \mathrm{mK}$, averaged over many traces of the type shown in panel (a) (Folk et al., 1996); solid line, the fitted squared-Lorentzian line shape (80) predicted by Alhassid and Attias (1996a); inset, the universal Gaussian unitary process correlator calculated by random-matrix process simulations (circles) compared with its squared-Lorentzian fit (80) with $\alpha_{2}=0.54$ (solid line). Adapted from Folk et al. (1996) and Alhassid and Attias (1996a).

The correlator $c_{g}(\Delta x)$ can be calculated perturbatively to leading order in $\Delta \bar{x}$ (Alhassid and Attias, 1996a, 1996b),

$c_{g}(\Delta x) \approx \begin{cases}1-b_{1}|\Delta \bar{x}|^{2} & \\ \times\left(-\frac{\pi^{2}}{6} \ln |\Delta \bar{x}|+\mathrm{const}\right) & (\beta=1) \\ 1-b_{2} \frac{\pi^{2}}{3}|\Delta \bar{x}|^{2} & (\beta=2)\end{cases}$ 
and for the Gaussian orthogonal process it is nonanalytic at $\Delta \bar{x}=0$. The constant $b_{\beta}$ in Eq. (131) depends on the leads. For single-channel symmetric leads $b_{1}=7 / 4$ and $b_{2}=3$.

The peak-height autocorrelation versus a magnetic field was measured by Folk et al. (1996). The results are shown in Fig. 17(b) (diamonds) and compared with the theoretical prediction (80) of Alhassid and Attias (1996a; solid line). Random-matrix theory predicts a universal correlator after the parameter is scaled. However, the scaling factor itself - for a particular choice of the parameter - cannot be computed in RMT. When $x$ is a magnetic field, we can rewrite Eq. (80) as

$$
c_{g}(\Delta B) \approx\left[1+\left(\Delta B / B_{c}\right)^{2}\right]^{-2}
$$

where $B_{c}$ is the correlation field. The curve in Fig. 17(b) represents a one-parameter fit (i.e., $B_{c}$ ) to the data. It is found that $B_{c}=8.1 \pm 0.5 \mathrm{mT}$ or $\Phi_{c} \approx 0.8 \Phi_{0}$.

The correlation field in closed dots can be estimated semiclassically, similarly to the open dot case (see Sec. IV.C.2). Since the decay time of a resonance in a closed dot is much longer than the Heisenberg time, the latter becomes the relevant time scale for the diffusive area accumulation. The estimate for $B_{c}$ in a closed dot is then obtained by replacing $\tau_{\text {escape }}$ in (102) with $\tau_{H}$ :

$$
B_{c} \mathcal{A} / \Phi_{0}=\kappa\left(2 \pi \tau_{c} / \tau_{H}\right)^{1 / 2}=\kappa g_{T}^{-1 / 2}=\kappa 4 \pi^{2} \mathcal{N}^{-1 / 4}
$$

where $g_{T}$ is the ballistic Thouless conductance. A semiclassical derivation can be found in Bohigas et al. (1995). In the conformal billiard with a flux line (Berry and Robnik, 1986) $\Phi_{c} \approx 0.1 \Phi_{0}$, but for a stadium in a uniform magnetic field (Bohigas et al., 1995) flux is accumulated less efficiently and $\Phi_{c} \approx 0.3 \Phi_{0}$ (Alhassid, Hormuzdiar, and Whelan, 1998). This is still below the experimental value $\Phi_{c} \approx 0.8 \Phi_{0}$, indicating that the single-particle picture is inadequate for estimating the correlation field. We shall return to this problem in Sec. VI.C.

\section{Crossover from conserved to broken time-reversal symmetry}

Following Alhassid, Hormuzdiar, and Whelan (1998), we derive in this section the peak-height statistics in the crossover between GOE and GUE. We use the crossover random-matrix ensemble (68), which is characterized by the transition parameter $\zeta$. When time-reversal symmetry is broken by a magnetic field, $\zeta=\Phi / \Phi_{c r}$, where $\Phi_{c r}$ is a characteristic crossover flux of the same order as the correlation field in Eq. (133).

\section{Conductance peak distributions}

Using the method of Sec. III.C.2, we decompose the partial amplitudes of an eigenfunction $\boldsymbol{\psi}$ in the principal frame: $\gamma_{c}=\gamma_{c R}+i \gamma_{c I}=\left\langle\boldsymbol{\phi}_{c} \mid \boldsymbol{\psi}_{R}\right\rangle+i\left\langle\boldsymbol{\phi}_{c} \mid \boldsymbol{\psi}_{I}\right\rangle$. The joint partial-width amplitude distribution is then given by

$$
\begin{aligned}
& P_{\zeta}(\gamma)=\langle P(\gamma \mid t)\rangle \\
& P(\gamma \mid t)=\left(\frac{1+t^{2}}{2 \pi t}\right)^{\Lambda}(\operatorname{det} M)^{-1} \\
& \times \exp \left(-\frac{1+t^{2}}{2} \gamma_{R}^{T} M^{-1} \gamma_{R}-\frac{1+t^{2}}{2 t^{2}} \gamma_{I}^{T} M^{-1} \gamma_{I}\right)
\end{aligned}
$$

where the brackets $\langle\ldots\rangle$ denote an average over the distribution $P_{\zeta}(t)$ in Eq. (71). Here $M$ is the correlation matrix [Eq. (122)] and is assumed to be independent of $\zeta$. This is correct as long as the dot-leads geometry is held fixed as the magnetic field is changed.

The width for decay into a one-channel lead is given by $\Gamma=|\gamma|^{2}=\gamma_{R}^{2}+\gamma_{I}^{2}$. Using Eq. (134) we find $P_{\zeta}(\hat{\Gamma})=$ $\left\langle a_{+} e^{-a_{+}^{2} \hat{\Gamma}} I_{0}\left(a_{+} a_{-} \hat{\Gamma}\right)\right\rangle$, where $a_{ \pm} \equiv\left(t^{-1} \pm t\right) / 2$, and $I_{0}$ is the modified Bessel function of order zero.

For the general case of $\Lambda$ inequivalent and/or correlated channels, we note that the joint conditional distribution $P(\gamma \mid t)$ in Eq. (134) is identical to the joint partial-width amplitude distribution for a GOE problem of $2 \Lambda$ channels with partial amplitudes $\gamma_{c R}, \gamma_{c I}$ and an extended correlation matrix $\mathcal{M}$ composed of four $\Lambda \times \Lambda$ blocks:

$$
\mathcal{M}=\left(\begin{array}{cc}
\frac{1}{1+t^{2}} M & 0 \\
0 & \frac{t^{2}}{1+t^{2}} M
\end{array}\right)
$$

We can therefore use the known GOE width and conductance peak distributions from Secs. V.B.2 and V.B.3. The $2 \Lambda$ eigenvalues of $\mathcal{M}$ are given by $\left\{\omega_{j}^{2}\right\}=$ $\left\{\frac{1}{1+t^{2}} w_{c}^{2}, \frac{t^{2}}{1+t^{2}} w_{c}^{2}\right\}$, where $w_{c}^{2}$ are the $\Lambda$ eigenvalues of $M$. Sorting the inverse eigenvalues of $\mathcal{M}$ in ascending order, $\omega_{1}^{-2}<\omega_{2}^{-2}<\ldots$, we find for the crossover width distribution

$$
\begin{aligned}
P_{\zeta}(\Gamma) & =\left\langle\frac{1}{\pi 2^{\Lambda}}\left(\prod_{c} \frac{1}{\omega_{c}}\right) \sum_{m=1}^{\Lambda}(-)^{m+1} \int_{1 / 2 \omega_{2 m-1}^{2}}^{1 / 2 \omega_{2 m}^{2}} d \tau\right. \\
& \left.\times \frac{\mathrm{e}^{-\Gamma \tau}}{\sqrt{\prod_{r=1}^{2 m-1}\left(\tau-\frac{1}{2 \omega_{r}^{2}}\right) \prod_{s=2 m}^{2 \Lambda}\left(\frac{1}{2 \omega_{s}^{2}}-\tau\right)}}\right\rangle .
\end{aligned}
$$

In the crossover regime $\Gamma^{l}$ and $\Gamma^{r}$ are no longer statistically independent, i.e., $P\left(\Gamma^{l}, \Gamma^{r}\right)=\left\langle P\left(\Gamma^{l} \mid t\right) P\left(\Gamma^{r} \mid t\right)\right\rangle \neq$ $\left\langle P\left(\Gamma^{l} \mid t\right)\right\rangle\left\langle P\left(\Gamma^{r} \mid t\right)\right\rangle=P\left(\Gamma^{l}\right) P\left(\Gamma^{r}\right)$. This is just the manifestation of the long-distance correlations in the transition statistics discovered by Fal'ko and Efetov (1996). On the other hand, at fixed $t, \Gamma^{l}$ and $\Gamma^{r}$ are independent, and $P(g \mid t)$ is calculated in closed form by following 
the same steps as for the GOE case (Alhassid, Hormuzdiar, and Whelan, 1998). The peak-height distributions $P_{\zeta}(\ln g)$ in the crossover from GOE to GUE are shown in Fig. 18. Here $\ln g$ is chosen as the variable in order to show the behavior at small intensities over several orders of magnitude (Alhassid and Levine, 1986). The left inset confirms the RMT predictions for the conformal billiard.

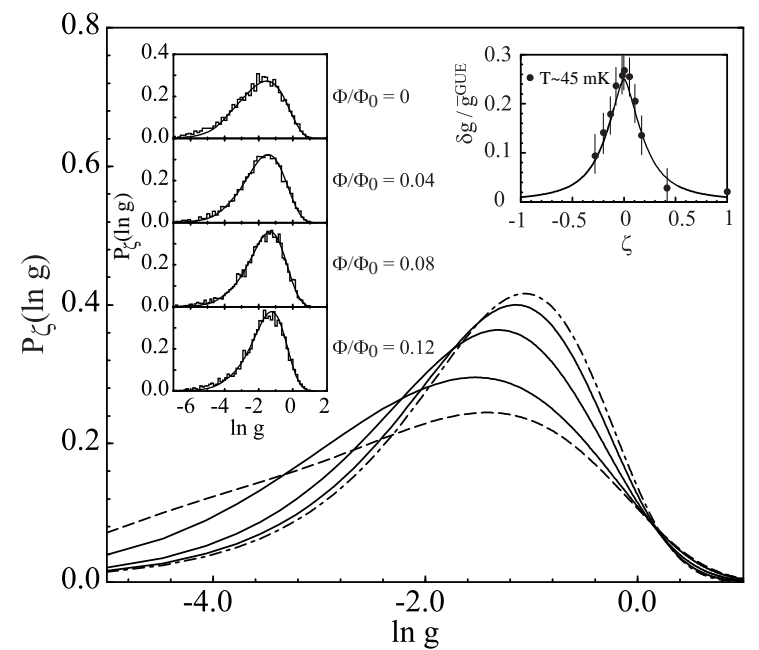

FIG. 18. Coulomb-blockade conductance peak-height distributions in the crossover from conserved to broken timereversal symmetry. The distributions for single-mode leads are shown vs $\ln g$ for $\zeta=0$ (GOE, dashed line), $\zeta=$ $0.1,0.25,0.5$ (solid lines), and $\zeta \gg 1$ (GUE, dot-dashed line). Left inset, distributions obtained from simulations of the conformal billiard threaded by different amounts of magnetic flux (histograms) are compared with theoretical distributions calculated for $\zeta \approx 4 \Phi / \Phi_{0}$ (solid lines). Right inset, the analytic weak-localization line shape $\delta g(\zeta) / \bar{g}^{\mathrm{GUE}}$ for a dot with singlechannel symmetric leads (solid line), compared with a recent experiment (solid circles) by Folk et al. (2000). The quantity $\bar{g}^{\mathrm{GUE}}$ is measured away from $B=0$ and $\zeta=B / B_{c r}$ with $B_{c r} \approx 6 \mathrm{mT}$. Adapted from Alhassid, Hormuzdiar and Whelan (1998) and Alhassid (1998).

\section{Weak localization}

We already know from Sec. V.B.3 that a weaklocalization correction is predicted for the average conductance peak height. The complete dependence of $\bar{g}$ on $\zeta$ (for symmetric leads with $\Lambda$ channels in each lead) was calculated in closed form by Alhassid (1998). The calculation is simpler than the corresponding one in open dots (see Sec. IV.C.2) and can be done within the framework of RMT. For a dot with single-channel symmetric leads $(\Lambda=1)$,

$$
\bar{g}(\zeta)=\frac{1}{4}+\left\langle\left(\frac{t}{1-t^{2}}\right)^{2}\left(\frac{2 t^{2}}{1-t^{4}} \ln t+\frac{1}{2}\right)\right\rangle .
$$

As in the case of open dots, we define $\delta g_{\Lambda}(\zeta) \equiv \bar{g}_{\Lambda}^{\mathrm{GUE}}-$
$\bar{g}_{\Lambda}(\zeta)$. The quantity $\delta g_{\Lambda}(\zeta)$ is largest at $\zeta=0(\mathrm{GOE}$ limit) where $\delta g_{\Lambda}(0) / \bar{g}_{\Lambda}^{\mathrm{GUE}}=1-\bar{g}_{\Lambda}^{\mathrm{GOE}} / \bar{g}_{\Lambda}^{\mathrm{GUE}}=1 /[2(\Lambda+$ $1)]$, and approaches zero for $\zeta \rightarrow \pm \infty$. The right inset of Fig. 18 shows that the predicted weak-localization line shape $\delta g_{\Lambda}(\zeta) / \bar{g}_{\Lambda}^{\mathrm{GUE}}$ for a dot with one-channel symmetric leads (solid line) agrees well with a recent experiment (Folk et al., 2000) after scaling of $B$.

In closed dots, the full width at half maximum (FWHM) of the weak-localization line shape is almost independent of the number of channels $\Lambda$, in contrast with open dots, where the FWHM behaves as $\sim \sqrt{\Lambda}$ for large $\Lambda$. This difference can be understood in terms of the different time scales involved. In open dots, the crossover in the average conductance occurs when $\tau_{\text {escape }} / \tau_{\text {mix }}=4 \pi^{2} \zeta^{2} / \Lambda^{2} \sim 1$, leading to $\zeta_{c r}^{\text {open }} \sim \sqrt{\Lambda} / 2 \pi$ [see the discussion following Eq. (103)]. In closed dots, on the other hand, the "escape" time (by tunneling) is much longer than the Heisenberg time $\tau_{H}$, and it is the latter that competes with the mixing time. Since $\tau_{H}$ is longer by a factor of $\Lambda$ than the escape time in an open dot with $\Lambda$ ideal channels, we conclude that the crossover in the average conductance in closed dots occurs when $\zeta_{c r}^{\text {closed }} \sim 1$, independent of the number of channels.

The variance of the conductance peak height in the crossover from GOE to GUE can also be calculated in closed form (Alhassid, 1998). For single-channel symmetric leads,

$$
\begin{aligned}
\overline{g^{2}}(\zeta)= & \frac{3}{16}+\frac{27}{2}\left\langle( \frac { t } { 1 + t ^ { 2 } } ) ^ { 2 } ( \frac { t } { 1 - t ^ { 2 } } ) ^ { 4 } \left[\frac{1+t^{2}}{1-t^{2}} \ln t\right.\right. \\
& \left.\left.+1+\frac{1}{12}\left(\frac{1-t^{2}}{t}\right)^{2}-\frac{2}{27}\left(\frac{1-t^{2}}{t}\right)^{4}\right]\right\rangle
\end{aligned}
$$

\section{E. Peak-spacing statistics}

The spacings $\Delta_{2}$ between successive Coulomb-blockade peaks are observed to fluctuate around an average charging energy that changes smoothly as more electrons are added to the dot (Sivan et al., 1996). In the constantinteraction model, $\Delta_{2}=e \alpha\left(V_{g}^{\mathcal{N}+1}-V_{g}^{\mathcal{N}}\right)=E_{\mathcal{N}+1}-$ $E_{\mathcal{N}}+e^{2} / C$ [see Eq. (27]. Thus if the single-particle states are not spin degenerate (but without treating the spin-up and spin-down manifolds as statistically independent), we expect a (shifted) Wigner-Dyson distribution of the peak spacings. A detailed discussion of the experimental results is postponed to Sec. VI.A. Here we remark only that the observed distribution does not have a WignerDyson shape but is closer to a Gaussian (Sivan et al., 1996; Simmel, Heinzel and Wharam, 1997; Patel, Cronenwett, et al., 1998).

Can Gaussian-like spacing distributions be explained in the constant-interaction-plus-RMT approach? It was pointed out by Vallejos, Lewenkopf and Mucciolo (1998) 
that deviations from a Wigner-Dyson distribution may be due to shape deformation of the dot as the gate voltage changes. At gate voltage $V_{g}^{\mathcal{N}}$, corresponding to the $\mathcal{N}$ th conductance peak, the shape of the dot is $x_{\mathcal{N}}$. However, at the degeneracy point $V_{g}^{\mathcal{N}+1}$ of the next peak, the shape of the dot has changed to $x_{\mathcal{N}+1}$. The spacing $\Delta_{2}$ is now given by $\Delta_{2}-e^{2} / C=E_{\mathcal{N}+1}\left(x_{\mathcal{N}+1}\right)-E_{\mathcal{N}}\left(x_{\mathcal{N}}\right)$, where $E_{\lambda}(x)$ are the single-particle energies of the dot with shape $x$. The generic variation of the energy levels with $x$ can be studied in the framework of the Gaussian process (see Sec. III.C.3). Measuring all energies in units of the average level spacing $\Delta$, we have for $\tilde{\Delta}_{2} \equiv\left(\Delta_{2}-e^{2} / C\right) / \Delta$

$$
\begin{aligned}
\tilde{\Delta}_{2} & =\left[\epsilon_{\mathcal{N}+1}\left(x_{\mathcal{N}+1}\right)-\epsilon_{\mathcal{N}}\left(x_{\mathcal{N}+1}\right)\right]+\left[\epsilon_{\mathcal{N}}\left(x_{\mathcal{N}+1}\right)-\epsilon_{\mathcal{N}}\left(x_{\mathcal{N}}\right)\right] \\
& \equiv \Delta \epsilon^{(\mathcal{N}+1)}+\Delta \epsilon_{\mathcal{N}}
\end{aligned}
$$

where $\Delta \epsilon^{(\mathcal{N}+1)}$ denotes the spacing between successive levels in a dot with a fixed shape $x_{\mathcal{N}+1}$, while $\Delta \epsilon_{\mathcal{N}}$ denotes the parametric variation of the $\mathcal{N}$-th level as the shape of the dot changes between peaks. The parametric fluctuations of the levels are universal once $x$ is scaled according to Eq. (77). Defining $\delta x_{\mathcal{N}} \equiv x_{\mathcal{N}+1}-x_{\mathcal{N}}$, we assume that the scaled $\delta \bar{x}_{\mathcal{N}} \approx \delta \bar{x}$ is independent of $\mathcal{N}$ $(\delta \bar{x}$ is the parametric distance $\delta x$ measured in units of the average distance between avoided level crossings). The distributions $P\left(\tilde{\Delta}_{2}\right)$ are then universal and depend only on $\delta \bar{x}$ and the symmetry class. Figure 19(c) shows the standard deviation of the spacings $\sigma\left(\tilde{\Delta}_{2}\right)$ versus $\delta \bar{x}$. It increases with $\delta \bar{x}$, i.e., the peak-spacing fluctuations are larger when the single-particle spectrum changes faster with the addition of electrons into the dot. The inset in Fig. 19(c) shows the GOE $\sigma\left(\tilde{\Delta}_{2}\right)$ (solid line) in comparison with $\left[\overline{\left(\Delta \epsilon_{\mathcal{N}}\right)^{2}}\right]^{1 / 2}$ (the level-diffusion correlator calculated in Sec. III.C.3). The $\sigma\left(\tilde{\Delta}_{2}\right)$ curve interpolates between the Wigner-Dyson value at $\delta \bar{x}=0$ and $\left[\overline{\left(\Delta \epsilon_{\mathcal{N}}\right)^{2}}\right]^{1 / 2}$ at large $\delta \bar{x}$.

For small $\delta \bar{x}$, the distributions can be calculated in closed form. Using the Gaussian process (78) near $x=0$, we find that $\Delta \epsilon_{\mathcal{N}} \approx\left\langle\psi_{\mathcal{N}}\left|H_{2}\right| \psi_{\mathcal{N}}\right\rangle \delta x$, where $\psi_{\mathcal{N}}$ is an eigenfunction of $H_{1}$. At fixed $H_{1}, \Delta \epsilon_{\mathcal{N}}$ is thus a Gaussian variable with zero mean and variance of $(\delta \bar{x})^{2}$, i.e., $P\left(\Delta \epsilon_{\mathcal{N}}\right)=(2 \pi)^{-1 / 2}(\delta \bar{x})^{-1} \exp \left[-\left(\Delta \epsilon_{\mathcal{N}}\right)^{2} / 2(\delta \bar{x})^{2}\right]$. Following Eq. (138), we convolute this Gaussian with the Wigner-Dyson distribution $P_{\mathrm{WD}}(s)$ [see Eq. (62)] to find

$$
P\left(\tilde{\Delta}_{2}\right) \approx(2 \pi)^{-1 / 2} \int_{0}^{\infty} d s P_{\mathrm{WD}}(s)(\delta \bar{x})^{-1} e^{-\frac{\left(\tilde{\Delta}_{2}-s\right)^{2}}{2(\delta \bar{x})^{2}}}
$$

The distributions for finite $\delta \bar{x}$ are easily calculated by simulations. Figures $19(\mathrm{a})$ and $19(\mathrm{~b})$ show the peakspacing distributions (histograms) for $\delta \bar{x}=0.75$. Each of the distributions is compared with the Wigner-Dyson distribution of the same symmetry class (solid lines). The distributions are more Gaussian-like and have tails extending to negative spacings.
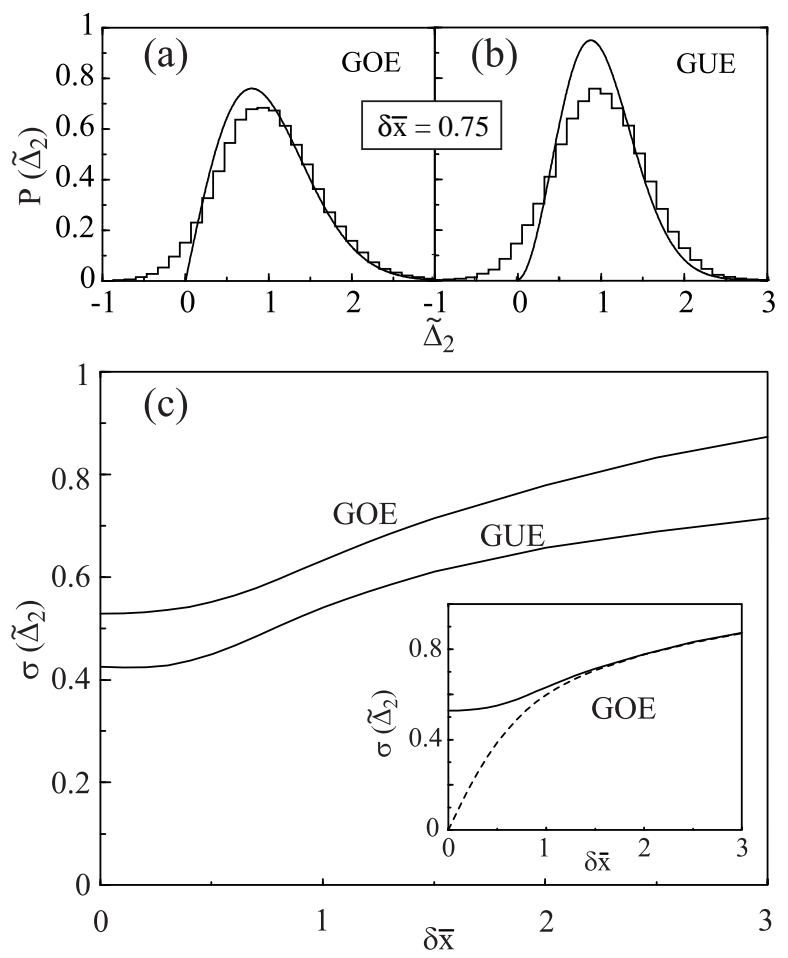

FIG. 19. Peak-spacing statistics and the parametric motion of energy levels: panels (a) and (b), the peak-spacing distribution $P\left(\tilde{\Delta}_{2}\right)$ (histograms) at $\delta \bar{x}=0.75$ for the (a) orthogonal and (b) unitary symmetries are compared with the WignerDyson nearest-neighbor spacing distribution for the respective symmetry (solid lines); (c) the standard deviation of the peak spacing $\sigma\left(\tilde{\Delta}_{2}\right)$ as a function of $\delta \bar{x}$ for both the GOE and GUE statistics. Inset in panel (c), $\sigma\left(\tilde{\Delta}_{2}\right)$ (solid line) compared with $\left[\overline{\left(\Delta \epsilon_{\mathcal{N}}\right)^{2}}\right]^{1 / 2}$, the rms of the change of a given energy level (dashed line). The latter is just the level-diffusion correlator shown in Fig. 10(c).

We shall return to the subject of peak-spacing statistics in the context of finite temperature (Sec. V.F.2) and interaction effects (Sec. VI).

\section{F. Finite-temperature statistics}

At finite temperatures that are not much smaller than $\Delta$, several resonances in the dot may contribute to the same conductance peak owing to the thermal smearing of the electron energy in the leads. The charging energy $E_{C}$ plays an important role. When $E_{C} \gg \Delta$, only two manifolds of the many-electron energy levels with $\mathcal{N}$ and $\mathcal{N}-1$ electrons in the dot contribute significantly since all other manifolds are pushed away by the charging energy. The rate-equations theory of Beenakker (1991) discussed in Sec. II.E takes into account this charging energy effect, 
and was used by Alhassid, Gökçedağ, and Stone (1998) to extend the statistical theory of closed dots to finite temperature.

\section{Conductance peaks}

In Sec. II.E we saw that the finite-temperature conductance is a weighted average of the single-level conductances: $g=\sum_{\lambda} w_{\lambda}\left(T, \tilde{E}_{F}\right) g_{\lambda}$, with thermal weights $w_{\lambda}$ given by Eq. (33) for $T \ll e^{2} / C$. These thermal weights depend on the canonical free energy $F_{\mathcal{N}}$ and canonical occupation numbers $\left\langle n_{\lambda}\right\rangle_{N}$. The latter are calculated exactly using particle-number projection (Ormand et al., 1994):

$$
\begin{aligned}
Z_{\mathcal{N}}= & e^{-F_{\mathcal{N}} / T}=\frac{e^{-\beta E_{0}}}{N_{s p}} \\
& \times \sum_{m=1}^{N_{s p}} \prod_{i=1}^{N_{s p}}\left(1+e^{-\beta\left|E_{i}-\mu\right|} e^{i \sigma_{i} \phi_{m}}\right), \\
\left\langle n_{\lambda}\right\rangle_{\mathcal{N}}= & \frac{e^{-\beta E_{0}}}{N_{s p} Z_{\mathcal{N}}} \sum_{m=1}^{N_{s p}}\left[\prod_{i=1}^{N_{s p}}\left(1+e^{-\beta\left|E_{i}-\mu\right|} e^{i \sigma_{i} \phi_{m}}\right)\right] \\
& \times \frac{1}{1+e^{\beta\left(E_{\lambda}-\mu\right)} e^{i \phi_{m}}},
\end{aligned}
$$

where the quadrature points are $\phi_{m}=2 \pi m / N_{s p}\left(N_{s p}\right.$ is the number of single-particle states), and $E_{0}=\sum_{i} E_{i}$. The quantity $\mu$ is a chemical potential chosen anywhere in the interval $E_{\mathcal{N}} \leq \mu<E_{\mathcal{N}+1} ; \sigma_{i}=1$ for a hole $\left(E_{i} \leq \mu\right)$ and -1 for a particle $\left(E_{i}>\mu\right)$.

The effect of energy-level fluctuations on the conductance statistics is small, and one can use a picket-fence spectrum to demonstrate the results. An example is shown in panels (a) and (b) of Fig. 20 for $T=0.5 \Delta$. The canonical occupation numbers as a function of $E_{\lambda}$ follow a curve that is similar to a Fermi-Dirac distribution with a chemical potential of $\left(E_{\mathcal{N}}+E_{\mathcal{N}+1}\right) / 2$ but with an effective temperature smaller by almost a factor of 2 in the vicinity of the chemical potential [see inset of Fig. 20(b)]. These results are in agreement with estimates by Kamenev and Gefen (1997). The thermal weights [Eq. (33] are shown in Fig. 20(a) as a function of the effective Fermi energy for several levels in the vicinity of the central level through which the tunneling occurs at low temperatures (denoted in the following by $\lambda=0)$. The ratio of the thermal weights $w_{\lambda}\left(T, E_{0}\right)$ to the noninteracting weights [Eq. (34)] is shown in Fig. 20 (b). For all $\lambda \neq 0$, we have $w_{\lambda} / w_{\lambda}^{(0)} \approx 1 / 2$ to within $20 \%$ or better. On the other hand, the ratio for the central level $w_{0} / w_{0}^{(0)}=\left\langle n_{0}\right\rangle>1 / 2$ is enhanced with respect to that for other levels. This enhancement causes the distribution of the conductance peaks (see below) to be less sensitive to temperature than what we would expect from a non-interacting theory. For temperatures above $\sim 2-3 \Delta,\left\langle n_{0}\right\rangle \approx 1 / 2$ and the conductance peak approaches $G \approx G^{(0)} / 2$ (Beenakker, 1991), where $G^{(0)}$ is the classical conductance in the absence of Coulomb blockade (see the discussion at the end of Sec. II.E).
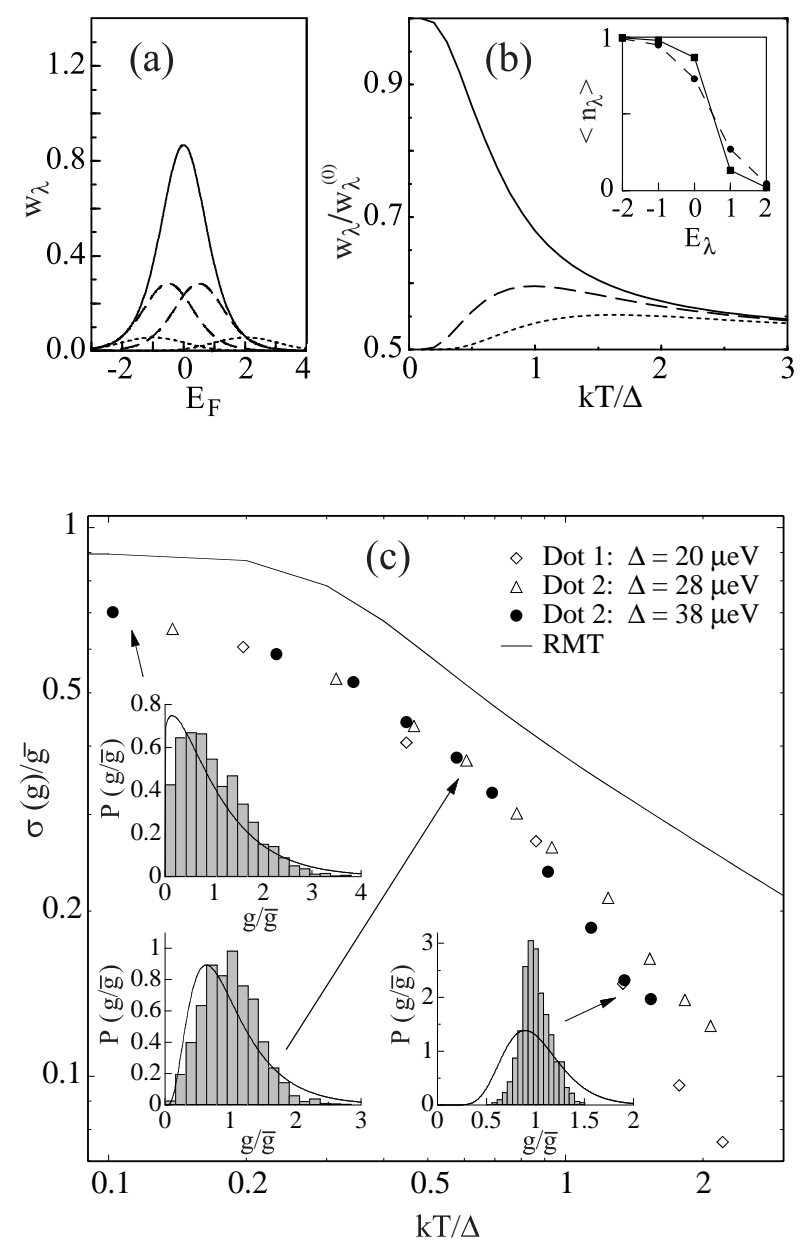

FIG. 20. Thermal weights of level conductances and finitetemperature statistics of the conductance peaks: (a) thermal weights $w_{\lambda}\left(T, \tilde{E}_{F}\right)$ of several level conductances vs Fermi energy $\tilde{E}_{F}$ at $k T=0.5 \Delta$; (b) ratio of thermal weights to their non-interacting values $w_{\lambda} / w_{\lambda}^{(0)}$ at $\tilde{E}_{F}=E_{0}$ vs $k T / \Delta$. Solid lines, $\lambda=0$; dashed lines, $\lambda= \pm 1$; dotted lines, $\lambda= \pm 2$. The results are for a picket-fence spectrum. The inset in (b) shows the canonical occupations (solid squares) compared with the Fermi-Dirac distribution at $k T=0.5 \Delta$ (dashed line). Adapted from Alhassid, Gökçedağ and Stone (1998); (c) the measured ratio $\sigma(g) / \bar{g}$ between the standard deviation of the peak-height fluctuations and the average peak height for three dots (symbols), compared with the RMT results (solid curve). The insets compare several of the measured distributions (histograms) with the finite-temperature RMT distributions (solid lines). From Patel, Stewart, et al. (1998).

\section{a. Distributions}

Complete RMT simulations show that spectral fluctuations have a small effect on the finite-temperature 
peak-height distribution $P(g)$ (Alhassid, Gökçedağ, and Stone, 1998). Closed expressions for $P(g)$ can be obtained if these fluctuations are ignored. In RMT, eigenfunctions that belong to different levels are uncorrelated and $P\left(g_{1}, g_{2}, \ldots\right)=\prod_{\lambda} P\left(g_{\lambda}\right)$, where $P\left(g_{\lambda}\right)$ is the distribution of a single-level conductance, derived in Sec. V.B. It follows that for a fixed sequence of energy levels, the characteristic function of the conductance peak distribution $P(u) \equiv \int_{0}^{\infty} d g e^{i u g} P(g)$ factorizes. Using the known single-level conductance distributions Eqs. (128a) and (128b), we find

$$
P(u)=\left\{\begin{array}{l}
\prod_{\lambda}\left(1-\frac{i u w_{\lambda}}{2}\right)^{-1 / 2} \\
\prod_{\lambda} \frac{1}{2\left(1-\frac{i u w_{\lambda}}{4}\right)} \\
\quad \times\left[1+\frac{\arcsin \left(\frac{i u w_{\lambda}}{4}\right)^{1 / 2}}{\left(\frac{i u w_{\lambda}}{4}\right)^{1 / 2}\left(1-\frac{i u w_{\lambda}}{4}\right)^{1 / 2}}\right]
\end{array}\right.
$$

Figure 20(c) compares recent experimental results by Patel, Stewart, et al. (1998) for the temperature dependence of $\sigma(g) / \bar{g}$ with the RMT predictions (solid line). The observed fluctuations exhibit a similar temperature dependence, but are smaller than the RMT predictions. Also shown are some of the experimental distributions (histograms) in comparison with the RMT distributions (solid lines). The deviations are larger at higher temperature, suggesting that they might be due to decoherence effects. Finite-temperature phase-breaking effects on the conductance of closed dots have not yet been studied.

\section{b. Peak-to-peak correlations}

The measured distributions of the Coulomb-blockade peak heights at low temperatures have confirmed the predictions of the statistical theory (see Sec. V.B.3). However, one of these experiments (Folk et al., 1996) also produced a puzzle: neighboring peaks are observed to be correlated [see Fig. 7(c)] although in RMT different eigenfunctions are uncorrelated. Since the temperature in this experiment is only $\sim 0.3-0.5 \Delta$, some of these correlations might be due to the finite temperature, where several resonances contribute to the same peak. We define the peak-to-peak correlator

$$
c(n)=\overline{\delta G_{\mathcal{N}} \delta G_{\mathcal{N}+n}} / \overline{\left(\delta G_{\mathcal{N}}\right)^{2}},
$$

where $\delta G_{\mathcal{N}}=G_{\mathcal{N}}-\bar{G}_{\mathcal{N}}$ is the fluctuation of the $\mathcal{N}$ th conductance peak around its average. An approximate expression for $c(n)$ is obtained by assuming that the location of the $\mathcal{N}$ th peak is fixed at its low-temperature value, $\tilde{E}_{F}=E_{\mathcal{N}}$ [note that $\tilde{E}_{F}$ is measured relative to $\left.(\mathcal{N}-1 / 2) e^{2} / C\right]$. Since eigenvectors and eigenvalues are uncorrelated in RMT, and using $\overline{g_{\lambda} g_{\mu}}=\overline{g_{\lambda}^{2}} \delta_{\lambda \mu}+\bar{g}_{\lambda}^{2}(1-$ $\left.\delta_{\lambda \mu}\right)$, we find

$$
c(n) \approx \frac{\overline{\sum_{\lambda} w_{\lambda}(\mathcal{N}+n) w_{\lambda}(\mathcal{N})}}{\overline{\sum_{\lambda} w_{\lambda}^{2}(\mathcal{N})}}
$$

where $w_{\lambda}(\mathcal{N}) \equiv w_{\lambda}\left(T, E_{\mathcal{N}}\right)$, and the remaining average is over the spectrum. Equation (143) can be simplified for a picket-fence spectrum: $c(n) \approx \sum_{\lambda} w_{\lambda-n} w_{\lambda} / \sum w_{\lambda}^{2}$, where $w_{\lambda}$ are the weights for a fixed number of electrons in the dot. The number $n_{c}$ of correlated peaks, defined as the FWHM of the correlator $c(n)$, is shown in Fig. 21(c) versus $T / \Delta$ and compared with a recent experiment by Patel, Stewart, et al. (1998) for three dots of different size. [The measured correlators $c(n)$ are shown in Figs. 21(a) and (b) for two of the dots.] While the qualitative increase of correlations with temperature is confirmed, we see enhanced correlations in the low temperature data. This is not fully understood, although several possible explanations were suggested:

(i) The correlations are enhanced because of spin-paired levels. Such levels can be identified by their similar magnetoconductance traces.
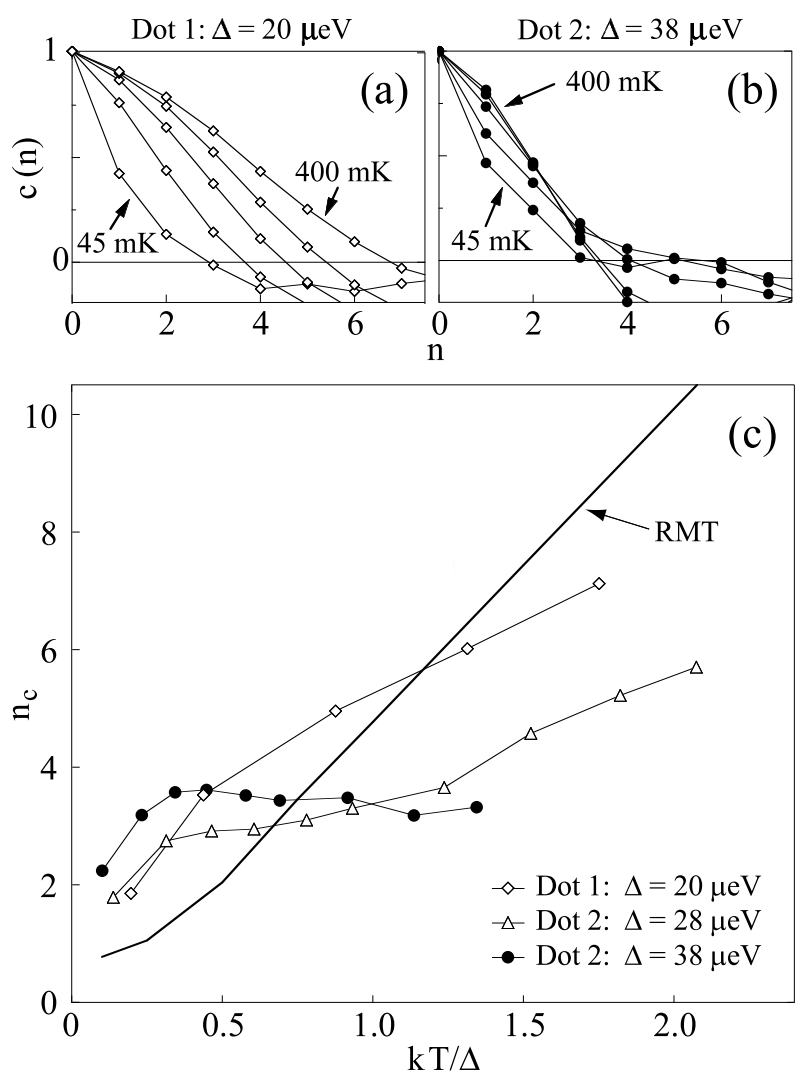

FIG. 21. Finite-temperature peak-to-peak correlations and spectral scrambling (experiment). The peak-to-peak correlator $c(n)$ is shown at different temperatures for (a) a larger dot with $\Delta=20 \mu \mathrm{eV}$ and (b) a smaller dot with $\Delta=38 \mu \mathrm{eV}$ (right). Notice that the correlator saturates sooner in the smaller dot. Compare with panels (a) and (b) of Fig. 23. (c) The number of correlated peaks $n_{c}$ as a function of $T / \Delta$ in three dots of different sizes. Also shown (solid line) are the RMT results for an unchanging spectrum. From Patel, Stewart, et al. (1998).

(ii) A mechanism was suggested (Hackenbroich, Heiss, 
and Weidenmüller, 1997; Baltin et al., 1999) whereby the change in deformation of the confining potential upon the addition of an electron into the dot results in a level crossing between successive Coulomb-blockade peaks such that the next added electron is essentially filling the same state. This can lead to a series of strongly correlated peaks. The mechanism assumes certain geometries (e.g., harmonic potentials) that are more suitable to nearly integrable dots. It remains to be seen whether this model can also explain enhanced correlations in "generic" chaotic dots.

(iii) A semiclassical theory of the Coulomb-blockade peak heights was discussed by Narimanov et al. (1999). The level width to decay into one of the leads is expanded as a sum over periodic orbits that are well coupled to the lead. A periodic modulation of the peak-height envelope is expected with a period of $\sim \hbar / \tau \Delta$, where $\tau=L / v_{F}$ is the period of the shortest orbit. For the dot used in the experiment of Patel, Stewart, et al. (1998), the estimated period corresponds to $\sim 12$ peaks, close to the experimental value of $\sim 15$. The peak heights have PorterThomas fluctuations only locally around the semiclassical envelope (Kaplan and Heller, 1998), but the resulting peak-height distribution is still found to be very close to the RMT distribution. The modulation also leads to enhanced correlations of adjacent peaks. However, this explanation requires certain geometries with periodic orbits that are strongly coupled to at least one of the leads.

An intriguing effect in the experimental results of Fig. 21 is the saturation of $n_{c}$ vs temperature at a value that depends on the dot's size. This effect will be explained in Sec. V.G.

\section{Peak spacings}

The finite-temperature statistical theory can also be used to calculate the temperature dependence of the peak-spacing distribution. Unlike the conductance peaks, the peak spacings are sensitive to the fluctuations of both the spectrum and the wave functions. While for $T \ll \Delta$ the peak height is located at $E_{\mathcal{N}}$, at temperatures of order $\Delta$ several levels contribute to a given peak, and fluctuations of the individual level conductances $g_{\lambda}$ may shift the peak location away from $E_{\mathcal{N}}$.

While we do not expect to reproduce the observed functional form of the distribution using a single-particle spectrum that is unchanged with the addition of electrons into the dot (see Sec. V.E), it is still of interest to understand the dependence of its width $\sigma\left(\tilde{\Delta}_{2}\right)$ on $T / \Delta$. Figure 22(a) shows a typical sequence of peak spacings at two different temperatures, demonstrating the decrease in peak-spacing fluctuations with temperature. Figure 22(b) compares the RMT result (solid line) for $\sigma_{\mathrm{GUE}}\left(\tilde{\Delta}_{2}\right)$ (Alhassid and Malhotra, 1999) with the experimental results of Patel, Cronenwett, et al. (1998) for $B \neq 0$.
Above $T / \Delta \sim 0.5$, we observe a sharp decrease of $\sigma\left(\tilde{\Delta}_{2}\right)$, in agreement with the experimental results. The inset is the calculated ratio $\sigma_{\mathrm{GOE}}\left(\tilde{\Delta}_{2}\right) / \sigma_{\mathrm{GUE}}\left(\tilde{\Delta}_{2}\right)$ as a function of $T / \Delta$. The experimental ratio $(\sim 1.2-1.3)$ measured at $T \sim 100 \mathrm{mK}$ is consistent with the calculations.
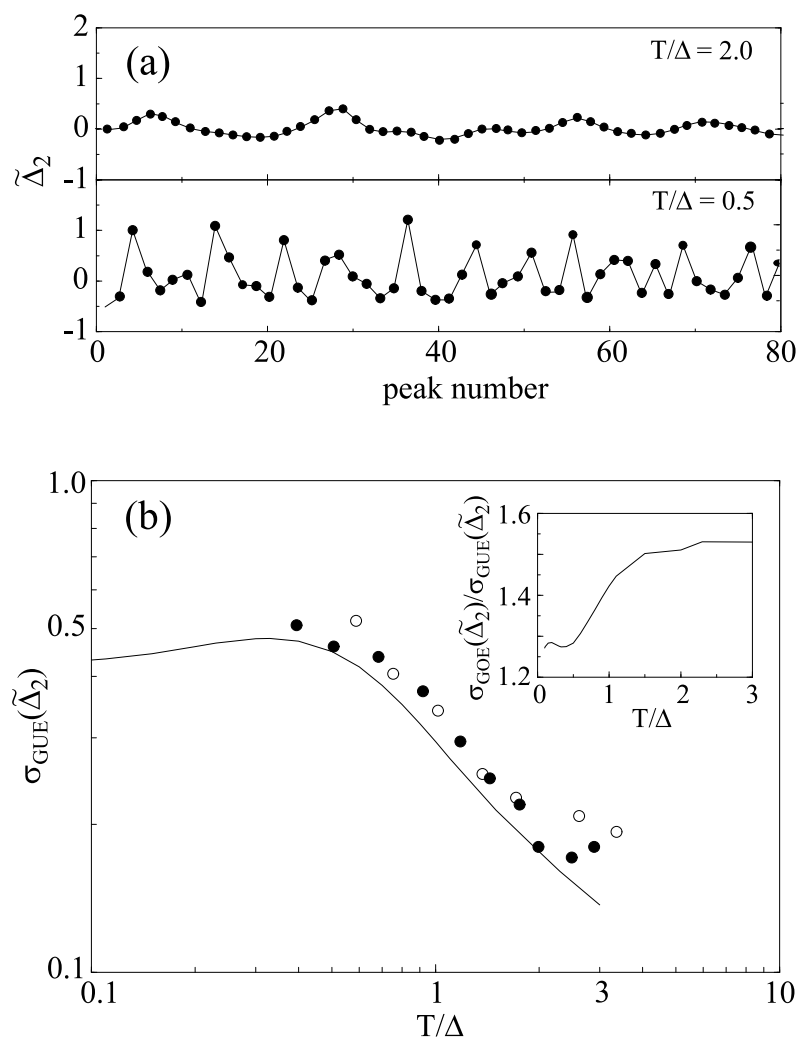

FIG. 22. Temperature dependence of the peak-spacing statistics in closed dots: (a) a sequence of peak spacings vs peak index at $T / \Delta=0.5$ (lower trace) and $T / \Delta=2$ (upper trace) obtained from one random-matrix realization; (b) the standard deviation $\sigma\left(\tilde{\Delta}_{2}\right)$ in the GUE statistics is shown versus $T / \Delta$ on a log-log scale. The theoretical RMT results (solid line) are compared with recent experimental data by Patel, Cronenwett, et al. (1998) taken at $B \neq 0$ for two dot configurations: solid circles, $\Delta=21 \mu \mathrm{eV}$; open circles, $\Delta=14 \mu \mathrm{eV}$. The charging energy is $E_{C}=590 \mu \mathrm{eV}$. The results are expressed in units of the mean level spacing $\Delta$. Inset, the ratio $\sigma_{\mathrm{GOE}}\left(\tilde{\Delta}_{2}\right) / \sigma_{\mathrm{GUE}}\left(\tilde{\Delta}_{2}\right)$ as a function of $T / \Delta$. From Alhassid and Malhotra (1999).

\section{G. Spectral scrambling}

In Sec. V.F, the finite-temperature statistics were discussed assuming that the single-particle spectrum is unchanged as electrons are added to the dot. However, in Sec. V.E we saw that a changing electronic spectrum has important effects on the $T \ll \Delta$ peak-spacing distribution. The single-particle spectrum is expected to change with the addition of electrons not only because of changes in the dot's shape, but, more importantly, be- 
cause of electron-electron interactions that lead to charge rearrangement on the dot. A detailed discussion of this point is postponed to Secs. VI.A.1 and VI.A.3, and here we simply assume that the changing spectrum can be modeled by a parametric dependence of an effective single-particle potential: the dot's Hamiltonian $H(x)$ depends on a parameter $x$ that assumes a discrete set of values $x_{\mathcal{N}}$ as electrons are added to the dot. From the theory of Gaussian processes (Sec. III.C.3), we expect the peak-to-peak correlator to be determined universally (for a fixed $T / \Delta$ ) by the value of the scaled parametric change $\delta \bar{x}$ between two successive peaks.
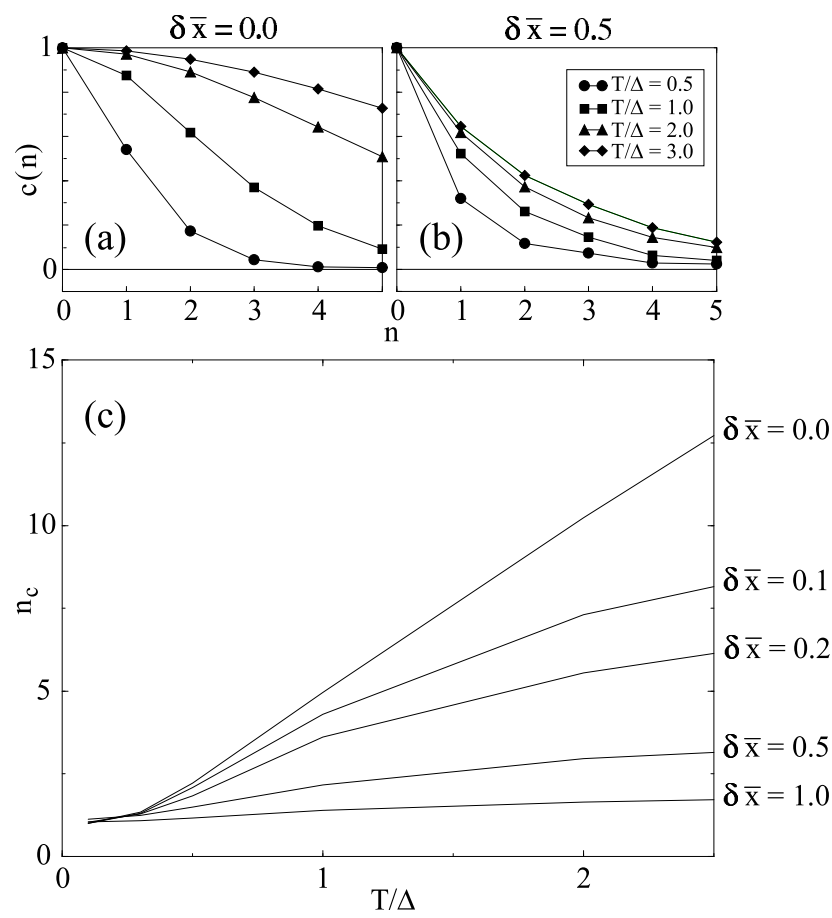

FIG. 23. Finite-temperature peak-to-peak correlations and spectral scrambling (theory). The peak-to-peak correlator $c(n)$ at $T / \Delta=0.5,1,2$, and 3 is shown for (a) a closed dot with a fixed spectrum $(\delta \bar{x}=0)$ and (b) a dot whose spectrum changes with the addition of electrons $(\delta \bar{x}=0.5)$. Here $\bar{\delta} x$ is a parameter characterizing the degree of statistical scrambling upon the addition of one electron to the dot. Notice the saturation of the correlator with temperature for the $\delta \bar{x} \neq 0$ case. (c) The number of correlated peaks $n_{c}$ [FWHM of $c(n)$ ] as a function of $T / \Delta$ for $\delta \bar{x}=0,0.1,0.2,0.5$, and 1 . The correlation length saturates sooner in a dot whose spectrum scrambles faster (i.e., in a dot with larger $\delta \bar{x}$ ). From Alhassid and Malhotra (1999).

Peak-to-peak correlations should be sensitive to a changing spectrum. For a fixed spectrum, the number of correlated peaks $n_{c}$ increases approximately linearly with $T$, as the number of levels that contribute to each peak is $\sim T / \Delta$. However, if the addition of each electron changes the spectrum, then $n_{c}$ is expected to saturate at a certain value $\sim m$ that measures the number of added electrons required to scramble the spectrum completely. These expectations are confirmed both experimentally and theoretically. Panels (a) and (b) of Fig. 23 show the calculated peak-to-peak correlator $c(n)$ at several temperatures for $\delta \bar{x}=0$ and $\delta \bar{x}=0.5$. We see that the correlator's width saturates in the $\delta \bar{x} \neq 0$ case. In Fig. 23(c) we show the number of correlated peaks $n_{c}$ as a function of temperature for several values of the scrambling parameter $\delta \bar{x}$. We observe that $n_{c}$ saturates at a value $m$ that decreases rapidly with increasing $\delta \bar{x}$.

Similar qualitative results are found in experiment (Patel, Stewart, et al., 1998). Figure 21(c) shows the experimental $n_{c}$ vs temperature for dots of different sizes. Also shown in Figs. 21(a) and 21(b) is the temperature dependence of the correlator $c(n)$ for two of the dots. Notice the similarity to the theoretical correlators of Figs. 23(a) and 23(b). We see that in the smallest device, saturation occurs at $m \sim 3-4$ already for $T \gtrsim 0.5 \Delta$, while for the larger dots, $n_{c}$ continues to increase with temperature. This suggests that spectral scrambling is slower in the larger dots. Indeed, it is argued in Sec. VI.A.3 that $\delta \bar{x}$ is smaller for a dot with a larger ballistic Thouless conductance $g_{T}$ [see Eqs. (158) below]. Since $g_{T} \propto \sqrt{\mathcal{N}}$, we expect the spectrum of the larger dots to have a smaller $\delta \bar{x}$ and thus to be less sensitive to the addition of electrons.

\section{H. Correlations between the addition and excitation spectra}

In the constant-interaction model, the ground state of a dot with $\mathcal{N}+n$ electrons is obtained by adding $n$ electrons to the first excited single-particle states of the $\mathcal{N}$ electron dot. On the other hand, we have seen in Sec. V.G that interactions scramble the single-particle spectrum when electrons are added to the dot, leading to the loss of correlations between the addition and excitation spectra. The measured finite-temperature peak-to-peak correlations shown in Fig. 21 suggest that a complete scrambling of the single-particle spectrum occurs only after several electrons $(\sim m)$ are added to the dot. We thus expect that for a small $n(\lesssim m)$, the ground state of a dot with $\mathcal{N}+n$ electrons is still correlated with the $n$th excited state of the dot with $\mathcal{N}$ electrons. The low-lying excited states in the dot can be observed through nonlinear transport experiments in the single-charge tunneling regime (see Sec . II.G).

Stewart et al. (1997) observed large correlations between the addition and excitation spectra up to $m \sim 4$. The strongest evidence for such correlations was observed in the magnetoconductance traces of the ground and excited levels in the dot. The height and position (in gate voltage $V_{g}$ ) of the differential conductance peak at finite source-drain voltage $V_{s d}$ could be followed as a function of magnetic field and compared with similar traces of the 
ground state in the linear Coloumb-blockade measurements. Stewart et al. found that the magnetoconductance trace of the $n$th excited states of an $\mathcal{N}$-electron dot was similar to the trace of the ground state of an $\mathcal{N}+n$-electron dot for $n<4$.

Another important result of the above experiment is the absence of spin degeneracy, contrary to the results observed in a few-electron dot (Tarucha et al., 1996; Kouwenhoven, Oosterkamp, et al., 1997). An excited level appears in the excitation spectrum for every electron that is removed from the dot.

\section{INTERACTION EFFECTS}

Electron-electron interactions - beyond the average interaction energy $\mathcal{N}^{2} e^{2} / 2 C$ of the constant-interaction model - are expected to play an important role in closed dots. Theoretical studies of interaction effects on the mesoscopic fluctuations in closed dots have been largely motivated by experiments showing deviations from the constant-interaction-plus-RMT model:

(i) The peak-spacing distributions (Sivan et al., 1996; Simmel, Heinzel, and Wharam, 1997; Patel, Cronenwett, et al., 1998; Simmel et al., 1999) do not have the WignerDyson form and their width is larger than expected from the constant-interaction model (see Sec. V.E).

(ii) The measured correlation flux of a conductance peak height is larger than its single-particle estimate (see Sec. V.C).

(iii) Correlations between the addition and excitation spectra diminish after the addition of a small number of electrons (see Sec. V.H).

(iv) The saturation of the peak-to-peak correlator with increasing temperature indicates spectral scrambling due to interactions (see Sec. V.G).

One way to include interaction effects while retaining a single-particle picture is in the Hartree-Fock approximation. Assuming that the Hartree-Fock singleparticle wave functions do not change upon the addition of an electron to the dot, Koopmans' theorem (Koopmans, 1934) states that the addition energy is given by the Hartree-Fock single-particle energy of the added electron. It is then possible to relate the peak spacing to the change in a single-particle Hartree-Fock level. This change is dominated by a certain diagonal interaction matrix element, which fluctuates due to the fluctuations of the single-particle wave functions. Blanter, Mirlin, and Muzykantskii (1997) used the random-phase approximation (RPA) to construct an effective screened potential from the bare Coulomb interaction in systems with finite geometries. They estimated the variance of a diagonal interaction matrix element, and find peak-spacing fluctuations that are larger but still of the order of the mean level spacing.
The RPA breaks down at strong interactions. Sivan et al. (1996) used an Anderson model of a disordered dot with electron-electron interactions to calculate numerically the the peak-spacing distribution. These calculations can be done only for a very small number of electrons (much fewer than in the experiments), but they explain the Gaussian shape of the distributions and yield larger widths for these distributions at stronger interactions. Berkovits and Sivan (1998) used the same model to study interaction effects on the peak-height statistics. Their results indicate that the peak-height distributions are only weakly sensitive to interactions but that the correlation field increases with interaction strength.

How does the electron's spin manifest itself in quantum dots? In the absence of interactions, the singleparticle states come in spin-degenerate pairs and the peak-spacing distribution is expected to be bimodal. No bimodality was seen in the experiments, an effect explained by strong electron-electron interactions (Berkovits, 1998). However, a recent experiment in dots with higher electron densities (where the Coulomb interactions are effectively weaker) showed spin-pairing effects in both the peak spacings and the parametric dependence of the peaks (Lüscher et al., 2000). The spin of the ground state and how it is affected by disorder or one-body chaos was the subject of recent theoretical investigations, and experimental results are expected in the near future.

The gas parameter $r_{s}$ measures the strength of the Coulomb interaction at an average distance between the electrons relative to their kinetic energy. It is universally determined by the density $n_{s}$ of the electron gas. In 2D, $\pi\left(r_{s} a_{B}\right)^{2}=1 / n_{s}$, where $a_{B}=\hbar^{2} / m^{*} e^{2}$ is the Bohr radius. The gas parameter $r_{s}$ is thus the radius, in atomic units, of the circle that encloses one unit of electron charge. The Fermi momentum is given by $k_{F} a_{B}=\sqrt{2} / r_{s}$, while the Fermi energy is $E_{F}=$ $\left(\hbar^{2} / 2 m^{*} a_{B}^{2}\right)\left(2 / r_{s}^{2}\right)$. The ratio between a typical Coulomb interaction energy $e^{2} / 2 r_{s} a_{B}$ and the average kinetic energy $E_{F} / 2$ is thus given by $r_{s} . r_{s}$ can also be expressed in terms of the Fermi velocity and the electron charge: $r_{s}=e^{2} / \hbar v_{F}$. The RPA is valid for $r_{s}<1$, but in typical semiconductor quantum dots $r_{s} \sim 1-2$.

In Sec. VI.A we discuss interaction effects on the peakspacing statistics using mean-field approximations and exact simulations. Spin effects are reviewed in Sec. VI.B and interaction effects on the peak-height statistics are discussed in Sec. VI.C. A random interaction matrix model is discussed in Sec. VI.D.

\section{A. Peak-spacing statistics and interactions}

The first experiment to measure the peak-spacing distribution in Coulomb-blockade quantum dots was carried out by Sivan et al. (1996). The spacing between 
successive Coulomb-blockade peaks is related to a second difference of the ground-state energy as a function of the number of electrons $\mathcal{N}$. To see that, we denote by $\mathcal{E}_{j}^{(\mathcal{N})}$ the ground-state energy of a dot with $\mathcal{N}$ electrons at a gate voltage $V_{g}(j)$ that corresponds to the degeneracy point of the $j-1 \rightarrow j$ transition. Since the average one-body potential induced by the gate is linear in the gate voltage $V_{g}$, the total energy of $\mathcal{N}$ electrons at the $j$ th peak is $\mathcal{E}_{j}^{(\mathcal{N})}-e \mathcal{N} \alpha V_{g}(j)$, where $\alpha$ is the ratio between the capacitance of the dot with respect to its gate and the total capacitance (see Sec. II.E). The degeneracy condition for the $\mathcal{N}+1$ peak is then $E_{F}+e \alpha V_{g}(\mathcal{N}+1)=\mathcal{E}_{\mathcal{N}+1}^{(\mathcal{N}+1)}-\mathcal{E}_{\mathcal{N}+1}^{(\mathcal{N})}$. The spacing between two consecutive peaks $\Delta V_{g}=V_{g}(\mathcal{N}+1)-V_{g}(\mathcal{N})$ is (Sivan et al., 1996)

$\Delta_{2}(\mathcal{N}+1) \equiv e \alpha \Delta V_{g}=\mathcal{E}_{\mathcal{N}+1}^{(\mathcal{N}+1)}+\mathcal{E}_{\mathcal{N}}^{(\mathcal{N}-1)}-\mathcal{E}_{\mathcal{N}+1}^{(\mathcal{N})}-\mathcal{E}_{\mathcal{N}}^{(\mathcal{N})}$

If the ground-state energy of the dot is independent of the gate voltage, then $\Delta_{2}=\mathcal{E}^{(\mathcal{N}+1)}+\mathcal{E}^{(\mathcal{N}-1)}-2 \mathcal{E}^{(\mathcal{N})}$. However, generally the change in the gate voltage is accompanied by a deformation of the $\operatorname{dot}$ so that $\mathcal{E}_{\mathcal{N}+1}^{(\mathcal{N})} \neq \mathcal{E}_{\mathcal{N}}^{(\mathcal{N})}$.

In the constant-interaction model, the ground-state en$\operatorname{ergy}$ is $\mathcal{E}_{j}^{(\mathcal{N})}=\mathcal{N}^{2} e^{2} / 2 C+\sum_{k=1}^{\mathcal{N}} E_{k}$ (where $E_{k}$ are the single-particle energies $)$, and $\Delta_{2}(\mathcal{N}+1)=E_{\mathcal{N}+1}-E_{\mathcal{N}}+$ $e^{2} / C$. Thus if we ignore the spin degrees of freedom, we expect $\tilde{\Delta}_{2} \equiv\left(\Delta_{2}-e^{2} / C\right) / \Delta$ to have a Wigner-Dyson distribution $P_{W D}\left(\tilde{\Delta}_{2}\right)$. The variance of $\Delta_{2}$ would then be $0.52 \Delta$ in the GOE and $0.42 \Delta$ in the GUE. This is the standard constant-interaction-plus-RMT model that we have used in earlier sections.

In the case of spin-degenerate single-particle states, we expect in the constant-interaction model $\Delta_{2}(\mathcal{N}+1)-$ $e^{2} / C=0$ for odd $\mathcal{N}$ (since two electrons with spin up and down can occupy the same level $\left.E_{(\mathcal{N}+1) / 2}\right)$, but $\Delta_{2}(\mathcal{N}+$ 1) $-e^{2} / C=E_{\mathcal{N} / 2+1}-E_{\mathcal{N} / 2}$ for even $\mathcal{N}$. The resulting distribution of $\tilde{\Delta}_{2}$ is bimodal:

$$
P\left(\tilde{\Delta}_{2}\right)=(1 / 2)\left[\delta\left(\tilde{\Delta}_{2}\right)+\frac{1}{2} P_{W D}\left(\frac{\tilde{\Delta}_{2}}{2}\right)\right]
$$

where the $\delta$ function and the Wigner-Dyson distribution originate in odd and even $\mathcal{N}$ 's, respectively. This is the constant-interaction-plus-spin-degenerate-RMT (CI + SDRMT) model, where the spacing for even $\mathcal{N}$ is on average larger by $2 \Delta$ than the spacing for odd $\mathcal{N}$, leading to a larger variance than in the constant-interactionplus-RMT model: $\sigma\left(\tilde{\Delta}_{2}\right)=1.24 \Delta$ for GOE and $1.16 \Delta$ for GUE.

Finally, another simple model considered in the literature is the constant-interaction-plus-spin-resolved-RMT model (CI+SRRMT). Here the assumption is that, because of exchange interactions, the different spin states are nondegenerate. However, the spin is a good quantum
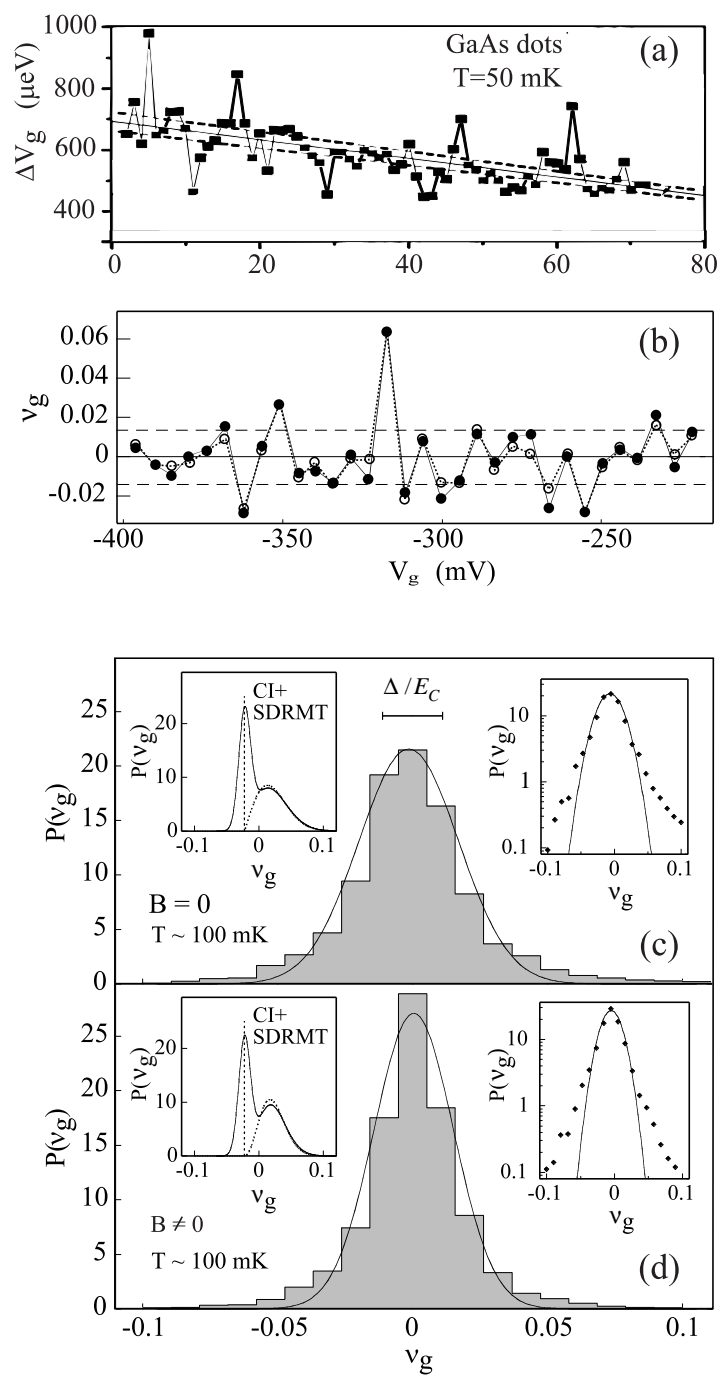

FIG. 24. Measured peak-spacing statistics in Coulombblockade dots: (a) peak-spacing series from Sivan et al. (1996) at $B=0$ showing $\Delta V_{g}$ between neighboring peaks vs the number of electrons $\mathcal{N}$ in the dot; solid line, linear fit describing the increase in capacitance with $\mathcal{N}$; (b) sequence of peakspacing fluctuations $\nu_{g} \equiv\left(\Delta V_{g}-\overline{\Delta V_{g}}\right) / \overline{\Delta V_{g}}$ vs $V_{g}$ from Patel, Cronenwett, et al. (1998); solid symbols, $B=30 \mathrm{mT}$; open symbols, $B=-30 \mathrm{mT}$. The dashed lines in panels (a) and (b) show the standard RMT deviation. Panels (c) and (d), spacing distributions $P\left(\nu_{g}\right)$ for $B=0$ and $B \neq 0$. The shaded histograms are the measured distributions at $T \sim 100 \mathrm{mK}$. The data contain 4300 peaks for $B=0$ of which 720 are statistically independent, and 10800 peaks for $B \neq 0$, of which 1600 are statistically independent. The data were collected at $T \sim 100 \mathrm{mK}$ from three dots with $\Delta=9-11 \mu \mathrm{eV}$ so that $T / \Delta \sim 0.8$. The solid lines are Gaussian fits. The right insets in (c) and (d) present the data and the fit on a log-linear scale to show deviations from Gaussians at the tails. The left insets in (c) and (d) are the bimodal constant-interaction-plus-spindegenerate-RMT distributions before (dotted lines) and after (solid lines) convolution with a Gaussian noise. From Patel, Cronenwett, et al. (1998). 
number, and therefore the subspaces of spin up and spin down are described by two independent randommatrix ensembles. The statistics of a superposition of two uncorrelated spectra were discussed by Dyson (1962b). The corresponding level-spacing distribution (see Mehta, 1991, Appendix A.2, p. 402) is characterized by a nonzero value at zero spacing. The corresponding widths $\sigma\left(\tilde{\Delta}_{2}\right)=0.70 \Delta$ for GOE and $0.65 \Delta$ for GUE are in between the values predicted by the $\mathrm{CI}+\mathrm{RMT}$ and CI+SDRMT models.

Sequences of peak spacings measured in gate voltage $\Delta V_{g}$ are shown in Figs. 24(a) and 24(b). The sequence in panel (a) is from Sivan et al. (1996), and the sequence in panel (b) is from Patel, Cronenwett, et al. (1998), where the quantity drawn is $\nu_{g} \equiv\left(\Delta V_{g}-\overline{\Delta V_{g}}\right) / \overline{\Delta V_{g}}$. The dashed lines describe the standard deviation of the Wigner-Dyson distribution. The fluctuations in Fig. 24(a) are significantly larger than in RMT, while those in 24(b) are somewhat larger than in RMT. The difference between the experiments in the magnitude of the fluctuations is not currently understood. The peak-spacing distribution is found to be more symmetric than in any of the above simple models and closer to a Gaussian. In particular, no bimodal structure is observed, suggesting the absence of spin degeneracy. An example of the observed distributions (histograms) is shown in panels (c) and (d) of Fig. 24 (Patel, Cronenwett, et al., 1998), where the solid lines are Gaussian fits. The scale of the mean level spacing is shown in units of the charging energy $E_{C}$. The ratio between the $B=0$ and $B \neq 0$ widths is $\sigma_{B=0}\left(\Delta_{2}\right) / \sigma_{B \neq 0}\left(\Delta_{2}\right) \approx 1.23$.

The discussion here is limited to almost-closed dots. The statistics of the peak spacings for a partially open dot were recently discussed by Kaminski and Glazman (2000), but only within the constant-interaction model. For a partially open dot, the peak position is affected by the dot-lead couplings, and the randomness of the electronic wave functions at the point contacts contribute to the peak-spacing fluctuations. This contribution increases with the strength of the dot-lead couplings and becomes comparable to the fluctuations of the singleparticle spacing while Coulomb-blockade peaks can still be observed.

In the following discussions of peak spacings and interactions, we ignore spin. Spin effects will be discussed in Sec. VI.B.

\section{Hartree-Fock approximation and Koopmans' theorem}

A consideration of interaction effects while retaining a single-particle picture is best done in the Hartree-Fock approximation. We start from a Hamiltonian of interacting electrons: $H=\sum_{i j} h_{i j} a_{i}^{\dagger} a_{j}+\frac{1}{4} \sum_{i j k l} v_{i j k l}^{A} a_{i}^{\dagger} a_{j}^{\dagger} a_{l} a_{k}$, where $h=t+V$ is the one-body part ( $V$ describes one-body disorder or a confining potential) and $v_{i j ; k l}^{A} \equiv$ $\langle i j|v| k l\rangle-\langle i j|v| l k\rangle$ are the antisymmetrized matrix elements of the two-body interaction. The single-particle Hartree-Fock energies and wave functions are determined self-consistently by solving the Hartree-Fock equations (see, e.g., Kittel, 1987). Denoting by $E_{k}^{(\mathcal{N})}$ the energy of the $k$ th single-particle state in a $\operatorname{dot}$ with $\mathcal{N}$ electrons, we have in the self-consistent basis

$$
E_{k}^{(\mathcal{N})}=h_{k k}+\sum_{i=1}^{\mathcal{N}} v_{k i ; k i}^{A},
$$

where the sum is over the $\mathcal{N}$ lowest occupied singleparticle states. The Hartree-Fock ground-state energy for $\mathcal{N}$ electrons is then given by $\mathcal{E}_{H F}^{(\mathcal{N})}=\sum_{k=1}^{\mathcal{N}} h_{k k}+$ $(1 / 2) \sum_{i, j=1}^{\mathcal{N}} v_{i j ; i j}^{A}$.

Numerical solutions of the Hartree-Fock equations will be discussed in Sec. VI.A.4. Here we use the framework of Koopmans' theorem (Koopmans, 1934), enabling us to relate the peak spacing to the single-particle HartreeFock energies. Koopmans' theorem states that

$$
\mathcal{E}_{H F}^{(\mathcal{N}+1)}-\mathcal{E}_{H F}^{(\mathcal{N})} \approx E_{\mathcal{N}+1}^{(\mathcal{N})}
$$

Its basic assumption is that the single-particle wave functions do not change when an electron is added. This assumption should hold only for large systems (Kittel, 1987), but it might be a good starting point for a dot with several hundred electrons. Under this assumption we find $\mathcal{E}_{H F}^{(\mathcal{N}+1)}-\mathcal{E}_{H F}^{(\mathcal{N})} \approx h_{\mathcal{N}+1, \mathcal{N}+1}+\sum_{i=1}^{\mathcal{N}} v_{\mathcal{N}+1, i ; \mathcal{N}+1, i}^{A}=E_{\mathcal{N}+1}^{(\mathcal{N})}$, which is just Eq. (147). Under the conditions of Koopmans' theorem we also have $E_{\mathcal{N}+1}^{(\mathcal{N})}=E_{\mathcal{N}+1}^{(\mathcal{N}+1)}$, and the peak spacing can be written as

$$
\Delta_{2}(\mathcal{N}+1) \approx E_{\mathcal{N}+1}^{(\mathcal{N}+1)}-E_{\mathcal{N}}^{(\mathcal{N})}=\Delta E^{(\mathcal{N}+1)}+\Delta E_{\mathcal{N}} .
$$

Here $\Delta E^{(\mathcal{N}+1)} \equiv E_{\mathcal{N}+1}^{(\mathcal{N}+1)}-E_{\mathcal{N}+1)}^{(\mathcal{N}+1)}$ measures the spacing between the $\mathcal{N}$ th and $\mathcal{N}+1$ st levels for a fixed number of electrons $(\mathcal{N}+1)$ and is of the order of the mean level spacing $\Delta$ (both levels are occupied and their spacing is expected to follow Wigner-Dyson statistics), while $\Delta E_{\mathcal{N}} \equiv E_{\mathcal{N}}^{(\mathcal{N}+1)}-E_{\mathcal{N}}^{(\mathcal{N})}$ is the change in energy of the same $\mathcal{N}$ th level when the number of electrons in the dot is increased from $\mathcal{N}$ to $\mathcal{N}+1$ :

$$
\begin{aligned}
\Delta E_{\mathcal{N}} & \approx v_{\mathcal{N}+1, \mathcal{N} ; \mathcal{N}+1, \mathcal{N}}^{A} \\
& =\int d \boldsymbol{r} d \boldsymbol{r}^{\prime}\left[\left|\psi_{\mathcal{N}}(\boldsymbol{r})\right|^{2} v\left(\boldsymbol{r}, \boldsymbol{r}^{\prime}\right)\left|\psi_{\mathcal{N}+1}\left(\boldsymbol{r}^{\prime}\right)\right|^{2}\right. \\
& \left.-\psi_{\mathcal{N}}^{*}(\boldsymbol{r}) \psi_{\mathcal{N}}\left(\boldsymbol{r}^{\prime}\right) v\left(\boldsymbol{r}, \boldsymbol{r}^{\prime}\right) \psi_{\mathcal{N}+1}(\boldsymbol{r}) \psi_{\mathcal{N}+1}^{*}\left(\boldsymbol{r}^{\prime}\right)\right] .
\end{aligned}
$$

The quantity $\Delta E_{\mathcal{N}}$ is of the order of the charging energy, and is a constant $e^{2} / C$ in the constant-interaction model. However, the fluctuations of the single-particle wave functions in Eq. (149) lead to fluctuations of $\Delta E_{\mathcal{N}}$ and can modify the peak-spacing statistics. 


\section{Random-phase approximation in disordered dots}

Blanter, Mirlin, and Muzykantskii (1997) calculated the fluctuations of $\Delta E_{\mathcal{N}}$ in Eq. (149) using the RPA and assuming a disordered dot in its metallic regime. The derivation consists of (i) calculating an effective interaction in the RPA to replace the bare Coulomb interaction in Eq. (149), and (ii) calculating the variance of the matrix element in Eq. (149) from the fluctuations of the single-particle eigenfunctions.

The Coulomb interaction $v_{0}\left(\boldsymbol{r}_{1}-\boldsymbol{r}_{2}\right)=e^{2} / \epsilon\left|\boldsymbol{r}-\boldsymbol{r}^{\prime}\right|$ (where $\epsilon$ is the dielectric constant) is screened. An effective screened potential $v\left(\boldsymbol{r}, \boldsymbol{r}^{\prime}\right)$ in the finite geometry of the dot can be found in the RPA (Fetter and Walecka, 1971):

$$
\begin{aligned}
v\left(\boldsymbol{r}, \boldsymbol{r}^{\prime}\right)= & v_{0}\left(\boldsymbol{r}-\boldsymbol{r}^{\prime}\right)-\int d \boldsymbol{r}_{1} d \boldsymbol{r}_{2} v_{0}\left(\boldsymbol{r}-\boldsymbol{r}_{1}\right) \\
& \times \Pi_{0}\left(\boldsymbol{r}_{1}, \boldsymbol{r}_{2}\right) v\left(\boldsymbol{r}_{2}, \boldsymbol{r}^{\prime}\right),
\end{aligned}
$$

where $\Pi_{0}$ is the static polarization function relating the electric potential fluctuation $\delta V$ to the charge density fluctuations $\delta \rho$ through $\delta \rho(\boldsymbol{r})=$ $-2 e \int d \boldsymbol{r}^{\prime} \Pi_{0}\left(\boldsymbol{r}, \boldsymbol{r}^{\prime}\right) \delta V\left(\boldsymbol{r}^{\prime}\right)$. In the limit where the screening length is large compared to the Fermi wavelength, the polarization function can be approximated by a localized function $\Pi_{0}\left(\boldsymbol{r}, \boldsymbol{r}^{\prime}\right) \approx \nu\left[\delta\left(\boldsymbol{r}-\boldsymbol{r}^{\prime}\right)-\mathcal{A}^{-1}\right]$, where $\nu$ is the density of states per unit area and $\mathcal{A}$ is the area of the dot. In an infinite system, Eq. (150) is easily solved by a Fourier expansion. In a finite geometry, the equations are solved by expanding in a complete set of eigenfunctions $\chi_{\lambda}(\boldsymbol{r})$ of the Laplacian in the dot with eigenvalues $q_{\alpha}^{2}$ that include the zero mode $\chi_{0}=1 / \mathcal{A}$. Three contributions to the effective potential are found:

$$
v\left(\boldsymbol{r}, \boldsymbol{r}^{\prime}\right)=e^{2} / C+\left[V_{\kappa}(\boldsymbol{r})+V_{\kappa}\left(\boldsymbol{r}^{\prime}\right)\right]+v_{\kappa}\left(\boldsymbol{r}, \boldsymbol{r}^{\prime}\right) .
$$

The first term is the usual charging energy, and the third term is the 2D screening potential $v_{\kappa}\left(\boldsymbol{r}, \boldsymbol{r}^{\prime}\right)=$ $\left(2 \pi e^{2} / \epsilon\right) \sum_{\alpha \neq 0}\left(q_{\alpha}+\kappa\right)^{-1} \chi_{\alpha}(\boldsymbol{r}) \chi_{\alpha}^{*}\left(\boldsymbol{r}^{\prime}\right)$, where $\kappa=$ $2 \pi e^{2} \nu / \epsilon$ is the inverse screening length. The new contribution in a finite geometry is a one-body potential, which for a disk of radius $R$ is $V_{\kappa}(\boldsymbol{r})=-\left(e^{2} / 2 \kappa R\right)\left(R^{2}-r^{2}\right)^{-1 / 2}$. This potential is the result of excess charge that is pushed to the boundaries of the dot: the added electron attracts a positive cloud around it, generating excess negative charge at the boundaries.

Using the effective interaction [Eq. (151)] in Eq. (149), the variation $\Delta E_{\mathcal{N}}$ of the $\mathcal{N}$ th Hartree-Fock level when an electron is added to the dot is composed of three contributions,

$$
\Delta E_{\mathcal{N}}=e^{2} / C+\Delta E_{\mathcal{N}}^{(1)}+\Delta E_{\mathcal{N}}^{(2)}
$$

corresponding to the three parts of the effective potential [Eq. (151)]. Fluctuations of $\Delta E_{\mathcal{N}}$ originate from fluctu- ations of the single-particle wave functions, and the variances $\sigma\left(\Delta E_{\mathcal{N}}^{(i)}\right.$ ) (where $i=1,2$ ) can then be expressed in terms of wave-function correlations. For example,

$$
\sigma^{2}\left(\Delta E_{\mathcal{N}}^{(1)}\right)=2 \int d \boldsymbol{r} d \boldsymbol{r}^{\prime} V_{\kappa}(\boldsymbol{r}) V_{\kappa}\left(\boldsymbol{r}^{\prime}\right) \overline{\left|\psi_{\mathcal{N}}(\boldsymbol{r})\right|^{2}\left|\psi_{\mathcal{N}}\left(\boldsymbol{r}^{\prime}\right)\right|^{2}}
$$

where we have ignored the exchange term in Eq. (149).

The universal RMT result for the correlation of the intensities of two eigenfunctions in chaotic systems is $\mathcal{A}^{2} \overline{\left|\psi_{\lambda}(\boldsymbol{r})\right|^{2}\left|\psi_{\mu}\left(\boldsymbol{r}^{\prime}\right)\right|^{2}}-1=\delta_{\lambda \mu}(2 / \beta) f_{d}^{2}\left(\left|\boldsymbol{r}-\boldsymbol{r}^{\prime}\right|\right)$, where $f_{d}$ is defined in Eq. (124). In diffusive systems $(l \ll L)$ this correlation is short-range (with a range of $\sim l$ ) because of an additional exponential factor $e^{-|\Delta r| / 2 l}$ in $f_{d}$. Long-range (diffuson) correlations are weaker by $1 / g_{T}$ but become important when integrated over in Eq. (153). Wave-function correlations in disordered systems were studied to order $1 / g_{T}$ by Blanter and Mirlin (1997) and reviewed by Mirlin $(1997,2000)$. For $\beta=1,2$ and eigenfunctions $\psi_{\lambda}, \psi_{\mu}(\lambda \neq \mu)$ whose energy separation is smaller than $E_{c}$, they find

$$
\begin{aligned}
& \mathcal{A}^{2} \overline{\left|\psi_{\lambda}(\boldsymbol{r})\right|^{2}\left|\psi_{\lambda}\left(\boldsymbol{r}^{\prime}\right)\right|^{2}}-1 \\
& =\frac{2}{\beta}\left[f_{d}^{2}\left(\left|\boldsymbol{r}-\boldsymbol{r}^{\prime}\right|\right)\left(1+\frac{2}{\beta} \Pi_{D}\left(\boldsymbol{r}, \boldsymbol{r}^{\prime}\right)\right)+\Pi_{D}\left(\boldsymbol{r}, \boldsymbol{r}^{\prime}\right)\right] \\
& \mathcal{A}^{2} \overline{\left|\psi_{\lambda}(\boldsymbol{r})\right|^{2}\left|\psi_{\mu}\left(\boldsymbol{r}^{\prime}\right)\right|^{2}}-1 \\
& =\frac{2}{\beta} f_{d}^{2}\left(\left|\boldsymbol{r}-\boldsymbol{r}^{\prime}\right|\right) \Pi_{D}\left(\boldsymbol{r}, \boldsymbol{r}^{\prime}\right) \\
& \mathcal{A}^{2} \frac{\psi_{\lambda}^{*}(\boldsymbol{r}) \psi_{\mu}(\boldsymbol{r}) \psi_{\lambda}\left(\boldsymbol{r}^{\prime}\right) \psi_{\mu}^{*}\left(\boldsymbol{r}^{\prime}\right)}{} \\
& =f_{d}^{2}\left(\left|\boldsymbol{r}-\boldsymbol{r}^{\prime}\right|\right)\left[1+(2-\beta) \Pi_{D}\left(\boldsymbol{r}, \boldsymbol{r}^{\prime}\right)\right]+\Pi_{D}\left(\boldsymbol{r}, \boldsymbol{r}^{\prime}\right),
\end{aligned}
$$

where $\Pi_{D}\left(\boldsymbol{r}, \boldsymbol{r}^{\prime}\right)=(\pi \nu)^{-1} \sum_{\alpha \neq 0} \chi_{\alpha}(\boldsymbol{r}) \chi_{\alpha}\left(\boldsymbol{r}^{\prime}\right) / D q_{\alpha}^{2}$ is the diffusion propagator expressed in terms of the eigenfunctions $\chi_{\alpha}(\boldsymbol{r})$ and eigenvalues $D q_{\alpha}^{2}$ of the diffusion operator. Here $\Pi_{D} \sim 1 / g_{T}$ is negligible at short distances compared with $f_{d}^{2}$, but dominates at long distances.

Using the correlator (154a) in Eq. (153), we find for the variance of $\Delta E_{\mathcal{N}}^{(1)}$

$$
\begin{aligned}
\sigma^{2}\left(\Delta E_{\mathcal{N}}^{(1)}\right)= & \frac{4}{\beta \mathcal{A}^{2}} \int d \boldsymbol{r} d \boldsymbol{r}^{\prime} V_{\kappa}(\boldsymbol{r})\left[f_{d}^{2}\left(\boldsymbol{r}-\boldsymbol{r}^{\prime}\right)\right. \\
& \left.+\Pi_{D}\left(\boldsymbol{r}, \boldsymbol{r}^{\prime}\right)\right] V_{\kappa}\left(\boldsymbol{r}^{\prime}\right)
\end{aligned}
$$

We now estimate the contribution to Eq. (155) of the short-range and long-range parts of the wave-function correlator. In 2D, $V_{\kappa} \sim e^{2} / \kappa \mathcal{A}$ over the whole dot while $\int d \boldsymbol{r} d \boldsymbol{r}^{\prime} f_{2}^{2}\left(\boldsymbol{r}-\boldsymbol{r}^{\prime}\right) \sim \mathcal{A} l / k_{F}$ and $\Pi_{D} \sim 1 / g_{T}$. The contribution of $f_{2}^{2}$ to Eq. (155) is then $\sim$ $\beta^{-1}\left(e^{2} / \epsilon \kappa \mathcal{A}\right)^{2} \mathcal{A l} / k_{F} \sim \beta^{-1}(l / L)^{2} \Delta^{2} / k_{F} l$, while the contribution of $\Pi_{D}$ is $\sim \beta^{-1} \Delta^{2} / g_{T}$. Since $g_{T} \sim k_{F} l$, we conclude that the short-range contribution is suppressed by a factor of $(l / L)^{2}$ relative to the long-range contribution, and 


$$
\begin{aligned}
\sigma^{2}\left(\Delta E_{\mathcal{N}}^{(1)}\right) & =\frac{4}{\beta \mathcal{A}^{2}} \int d \boldsymbol{r} d \boldsymbol{r}^{\prime} V_{\kappa}(\boldsymbol{r}) \Pi_{D}\left(\boldsymbol{r}, \boldsymbol{r}^{\prime}\right) V_{\kappa}\left(\boldsymbol{r}^{\prime}\right) \\
& \sim \frac{1}{\beta} \frac{\Delta^{2}}{g_{T}}
\end{aligned}
$$

A similar calculation of $\Delta E_{\mathcal{N}}^{(2)}$ using the two-body screened potential $v_{\kappa}\left(\boldsymbol{r}, \boldsymbol{r}^{\prime}\right)$ in Eq. (149) and the correlator (154b) gives

$$
\begin{aligned}
\sigma^{2}\left(\Delta E_{\mathcal{N}}^{(2)}\right) & \approx \frac{4 \Delta^{2}}{\beta^{2} \mathcal{A}^{2}} \int d \boldsymbol{r} d \boldsymbol{r}^{\prime} \Pi_{D}^{2}\left(\boldsymbol{r}, \boldsymbol{r}^{\prime}\right) \\
& \sim \frac{1}{\beta^{2}}\left(\frac{\Delta}{g_{T}}\right)^{2} .
\end{aligned}
$$

The charging energy $e^{2} / C$ also fluctuates because of small deviations $(\sim 1 / \kappa R)$ of the capacitance from its purely geometrical value (Berkovits and Altshuler, 1997; Blanter and Mirlin, 1998), leading to $\sigma\left(e^{2} / C\right) \sim$ $\left(\ln g_{T} / \beta\right)^{-1 / 2} \Delta / g_{T}$.

The largest contribution to $\sigma\left(\Delta E_{\mathcal{N}}\right)$ comes from the surface charge: $\sigma\left(\Delta E_{\mathcal{N}}\right) \sim \Delta / \sqrt{\beta g_{T}}$ [see Eq. (156)]. In its absence (e.g., using periodic boundary conditions), the dominating contribution is from the usual 2D screened interaction $\sigma\left(\Delta E_{\mathcal{N}}\right) \sim \Delta / \beta g_{T}$ [se Eq. (157)]. It was assumed that the added-electron charge is spread over the complete dot. In practice, this will depend on the geometry of the dot and the gates. An opposite extreme is when the added-electron charge is confined to an area whose linear dimension is $\sim \lambda_{F}$, leading to $\sigma\left(\Delta E_{\mathcal{N}}\right) \sim \Delta / \sqrt{\beta}$.

The standard deviation $\sigma\left(\Delta_{2}\right)$ is obtained by combining the usual RMT fluctuations $(\sim \Delta)$ with the fluctuations of $\Delta E_{\mathcal{N}}$ [see Eq. (148)]. This results in peakspacing fluctuations that are enhanced with respect to RMT, but still of the order of $\Delta$.

The dots used in the experiments are ballistic rather than diffusive, requiring a new estimate of the fluctuations using wave-function correlations in chaotic systems. The leading universal contribution to the correlations in 2D is $f_{2}^{2}$, whose envelope decays slowly like $1 / k_{F}\left|\boldsymbol{r}-\boldsymbol{r}^{\prime}\right|$ over the whole area of the dot, and leads to $\sigma\left(\Delta E_{\mathcal{N}}^{(1)}\right) \sim$ $\Delta / \sqrt{\beta k_{F} L}$. A similar estimate for $\sigma\left(\Delta E_{\mathcal{N}}^{(1)}\right)$ is obtained if we replace $g_{T}$ in the diffusive result [Eq. (156)] by its ballistic analog $k_{F} L \sim \mathcal{N}^{1 / 2}$ (since $l>L$ ). How important are nonuniversal contributions to the wavefunction correlator in the chaotic case? Blanter, Mirlin, and Muzykantskii (1998) derived the wave-function statistics in a billiard with diffusive surface scattering. They found $\mathcal{A}^{2} \overline{\left|\psi_{\lambda}(\boldsymbol{r})\right|^{2}\left|\psi_{\lambda}\left(\boldsymbol{r}^{\prime}\right)\right|^{2}}-1=(2 / \beta) \Pi_{B}\left(\boldsymbol{r}, \boldsymbol{r}^{\prime}\right)$, where $\Pi_{B}$ is the ballistic analog of the diffusion propagator. The contribution to $\Pi_{B}\left(\boldsymbol{r}, \boldsymbol{r}^{\prime}\right)$ from straight-line trajectories connecting $\boldsymbol{r}$ to $\boldsymbol{r}^{\prime}$ gives $\Pi_{B}=1 / k_{F}\left|\boldsymbol{r}-\boldsymbol{r}^{\prime}\right|$, which is just the smoothed version of the universal correlations $f_{2}^{2}\left(\boldsymbol{r}-\boldsymbol{r}^{\prime}\right)$. Evaluation of $\Pi_{B}$ beyond the universal part requires knowledge of the classical dynamics. Scars along periodic orbits can enhance $\Pi_{B}$ and lead to larger peak-spacing fluctuations (Stopa, 1998) in self-consistent density-functional calculations (Stopa, 1996). Analogous results for the wave-function correlations were derived for a purely ballistic chaotic dot using semiclassical methods (Hortikar and Srednicki, 1998).

\section{Parametric variation of the mean field}

As electrons are added to the dot, the Hartree-Fock potential changes owing to charge rearrangement caused by the two-body interaction. We denote the HartreeFock Hamiltonian for $\mathcal{N}$ electrons by $H\left(x_{\mathcal{N}}\right)$. Rather then solving the microscopic Hartree-Fock equations, we can adopt a "macroscopic" approach assuming that, for a chaotic dot, $H\left(x_{\mathcal{N}}\right)$ describes a discrete Gaussian process (Attias and Alhassid, 1995). In Koopmans' limit we can relate the peak spacing to the single-particle levels through Eq. (148). In particular, the quantity $\Delta E_{\mathcal{N}}$ describes a discrete parametric variation of the $\mathcal{N}$ th eigenvalue when the Hamiltonian changes from $H\left(x_{\mathcal{N}}\right)$ to $H\left(x_{\mathcal{N}+1}\right)$. Since a discrete Gaussian process can be embedded in a continuous Gaussian process $H(x)$ (Sec. III.C.3), we obtain the formulation already discussed in Sec. V.E. However, the conceptual difference is that here the parametric variation of the single-particle spectrum is due to interaction effects, while in Sec. V.E it originated from a deformation of the dot's shape. Experimental results (Patel, Stewart, et al., 1998) suggest that the primary cause of a changing single-particle spectrum is interactions.

The variation of the spectrum with the addition of one electron to the dot is described by the scrambling parameter $\delta \bar{x}$ (see Sec. V.G). This parameter can be determined from the dot's properties in the limit of Koopmans' theorem where the single-particle wave functions are unchanged. In the parametric approach, this limit corresponds to first-order perturbation theory, where $\sigma^{2}\left(\Delta E_{\mathcal{N}}\right)=\Delta^{2}(\delta \bar{x})^{2}$. Since $\Delta E_{\mathcal{N}}$ in the parametric approach corresponds to $\Delta E_{\mathcal{N}}^{(1)}+\Delta E_{\mathcal{N}}^{(2)}$ in the microscopic approach of Sec. VI.A.2 $\left(e^{2} / C\right.$ has been subtracted in both cases), we can compare $\sigma^{2}\left(\Delta E_{\mathcal{N}}\right)$ with Eq. (156) [or Eq. (157)] to find that (Alhassid and Malhotra, 1999; Alhassid and Gefen, 2000)

$$
\begin{aligned}
\delta \bar{x} & \sim\left(\beta g_{T}\right)^{-1 / 2} \sim \beta^{-1 / 2} \mathcal{N}^{-1 / 4} \\
\text { or } \quad \delta \bar{x} & \sim\left(\beta g_{T}\right)^{-1} \sim \beta^{-1} \mathcal{N}^{-1 / 2},
\end{aligned}
$$

where Eq. (158b) holds in the absence of surface charge. Equations (158) are valid in the regime where $\sigma^{2}\left(\Delta E_{\mathcal{N}}\right)$ is linear in $(\delta \bar{x})^{2}$, i.e., for $\delta \bar{x} \lesssim 0.3$. Complete scrambling (upon the addition of $m$ electrons) is expected when the overall parametric change is one avoided crossing, i.e., $m \delta \bar{x} \sim 1$. We conclude that $m \sim\left(\beta g_{T}\right)^{1 / 2}$ or $m \sim \beta g_{T}$ for Eqs. (158a) or (158b), respectively. 


\section{Anderson model with interactions}

The RPA estimate in interacting disordered systems gives a peak-spacing standard deviation of the order of $\Delta$. However, the RPA is valid in the limit of high densities $\left(r_{s} \ll 1\right)$ and breaks down at the lower electron densities in semiconductor quantum dots (where $r_{s} \sim 1-2$ ). Yet $r_{s}$ is still substantially below the limit of Wigner crystallization, and additional insight into this regime of intermediate interactions can be gained by numerical calculations. Exact numerical diagonalizations are possible only for dots with a very small number of electrons ( $\sim 10)$ on a lattice with $m \sim 20$ sites (compared with several hundred electrons in the experiment). Berkovits and Sivan (see Sivan et al., 1996) used a 2D Anderson model with on-site disorder and Coulomb interactions. The Hamiltonian is $H=H_{1}+H_{2}$, where $H_{1}$ is a one-body Anderson Hamiltonian [Eq. (46)], and $H_{2}=\frac{1}{2} U_{c} \sum_{i \neq j}\left(\left|\boldsymbol{r}_{i}-\boldsymbol{r}_{j}\right| / a\right)^{-1} a_{i}^{\dagger} a_{i} a_{j}^{\dagger} a_{j}$ describes the twobody Coulomb interaction between electrons on the lattice, where $U_{c}=e^{2} / a$ is the interaction strength over one lattice spacing. They used small lattices $(\sim 4 \times 5)$ with $\mathcal{N} \leq 13$ to study the distribution and variance of $\Delta_{2}$ as a function of the interaction strength $U_{c}$. As $r_{s} \sim(\pi m / \mathcal{N})^{1 / 2}\left(U_{c} / 4 t\right)$ increases from zero, the spacing distribution deviates from the Wigner-Dyson distribution and becomes approximately a Gaussian for $r_{s} \gtrsim 1$. The variance continues to increase with $r_{s}$, and for $r_{s} \gg 1$ they find $\sigma\left(\Delta_{2}\right) \propto\left\langle\Delta_{2}\right\rangle \approx e^{2} / C$ with a proportionality constant of about $0.1-0.2$.

Larger dots can be solved under certain approximations. The self-consistent Hartree-Fock approximation was used to calculate the ground state of dots with up to $\sim 100$ spinless electrons (Cohen, Richter, and Berkovits, 1999; Levit and Orgad, 1999; Walker, Montambaux, and Gefen, 1999). The single-particle orbits are computed self-consistently, allowing for configuration rearrangement as an electron is added to the dot. The dominant contribution to the peak spacing comes from the di-

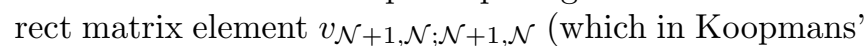
limit gives $\left.\Delta E_{\mathcal{N}}\right)$. The self-consistent Hartree-Fock calculations confirm that, for increasing $r_{s}$, the occupied (and unoccupied) levels exhibit Wigner-Dyson statistics, while the peak-spacing distribution evolves rapidly into a Gaussian-like distribution (at $r_{s} \sim 1$ ) with a width that is enhanced compared with the noninteracting picture. The gap in the Hartree-Fock spectrum between the highest filled level and lowest empty level is dominated by an interaction matrix element $v_{\mathcal{N}, \mathcal{N}+1 ; \mathcal{N}, \mathcal{N}+1}$ and is found to have a Gaussian-like distribution for large values of $r_{s}$.

Screening may not be very effective in the small dots used in the exact numerical simulations. Furthermore, screening can also be generated by external charges on nearby metallic plates. Consequently, Hartree-Fock simulations were also carried out for short-range (e.g., nearest-neighbor) interactions (Walker, Montambaux, and Gefen, 1999).

The dependence of the width $\sigma\left(\Delta_{2}\right)$ on the size of the dot is not fully understood. The RPA estimates predict $\sigma\left(\Delta_{2}\right) \propto \Delta$ for $r_{s} \ll 1$, while classical calculations by Koulakov, Pikus, and Shklovskii (1997) give $\sigma\left(\Delta_{2}\right) \propto$ $e^{2} / C \approx\left\langle\Delta_{2}\right\rangle$ for $r_{s} \gg 1$. The RPA scaling $\Delta \propto 1 / L^{2}$ has a different size dependence than the classical scaling $\left\langle\Delta_{2}\right\rangle \sim 1 / L$, a difference due to the delocalized character of the wave functions. It is not clear which is the correct scaling in the intermediate regime relevant to the experiments $\left(r_{s} \sim 1-2\right)$, although the self-consistent HartreeFock calculations (for Coulomb interactions) suggest typical fluctuations of $\sigma\left(\Delta_{2}\right) \sim 0.52 \Delta+a\left\langle\Delta_{2}\right\rangle / \sqrt{g_{T}}+\mathcal{O}\left(r_{s}^{2}\right)$, where $a$ is a constant (Bonci and Berkovits, 1999; Walker, Montambaux, and Gefen, 1999). For short-range interactions, a scaling of $\sim r_{s} \Delta$ is observed for $r_{s} \lesssim 1$.

The measured width of the spacing fluctuations shows substantial variation between different experiments. The experiments of Sivan et al. (1996), and Simmel, Heinzel, and Wharam (1997) gave $\sigma\left(\Delta_{2}\right) \approx(0.10-0.15) e^{2} / C \sim$ $(2-3) \Delta$, while Patel, Cronenwett, et al. (1998) found smaller fluctuations $\sigma\left(\Delta_{2}\right) \approx 0.05 e^{2} / C \sim \Delta$ for similar GaAs dots $\left(r_{s} \sim 1\right)$.

For short-range interactions Koopmans' theorem breaks down at $r_{s} \gtrsim 1$, and the self-consistent HartreeFock ground state develops charge-density modulations and increased short-range density correlations (Walker, Gefen, Montambaux, 1999). The addition spectrum shows nonuniversal features, and $\left\langle\Delta_{2}\right\rangle$ exhibits sharp maxima at certain magic numbers of $\mathcal{N}$, in agreement with a classical model of interacting charges. This result is quite surprising since $r_{s}$ is still much smaller than the Wigner crystal limit. A model of classical charges in a parabolic confining potential (Koulakov and Shklovskii, 1998) has explained the capacitance experiments of Zhitenev et al. (1997), where bunching was observed in the addition spectrum.

Nonuniversal effects were found in the addition spectra of clean chaotic dots for strong Coulomb interactions (Ahn, Richter, and Lee, 1999). They were explained by charge rearrangement that forms geometry-dependent ordered states localized at the edge of the dot.

\section{B. Spin effects and interactions}

In the presence of spin degrees of freedom, the singleparticle levels come in degenerate pairs of spin up and spin down with identical spatial wave functions. Though the Coulomb interaction does not depend explicitly on spin, the Pauli principle leads to an exchange interaction that favors larger spin values. Indeed, in a spin-polarized state, the orbital part of the wave function (having permutation symmetry conjugate to that of the spin part) is 
less symmetric, thus lowering the Coulomb repulsion. In the limit $g_{T} \gg 1$, it is possible to write a simple Hamiltonian for the dot (Kurland, Aleiner, and Altshuler, 2000). The fluctuations of the matrix elements in the disorder basis are $\mathcal{O}\left(1 / g_{T}\right)$ [see, for example, Eq. (157)], and in the limit $g_{T} \rightarrow \infty$ only the average diagonal interaction survives. We obtain

$$
H_{\mathrm{dot}}=\sum_{\lambda s} E_{\lambda} a_{\lambda s}^{\dagger} a_{\lambda s}+\frac{e^{2}}{2 C} \hat{\mathcal{N}}^{2}-\frac{1}{2} \xi \boldsymbol{S}^{2}
$$

where $\xi / 2 \equiv \bar{v}_{\alpha \beta ; \beta \alpha}$ is the average exchange matrix element and $S$ is the total spin operator of the dot. $a_{\lambda s}^{\dagger}$ and $a_{\lambda s}$ are creation and annihilation operators of an electron at orbital state $\lambda$ and spin $s= \pm 1 / 2$. Both interaction terms in Eq. (159) are invariant under a change of the single-particle basis (an additional Cooper-channel interaction is possible for the orthogonal symmetry, and is not included here). The single-particle part of Eq. (159) satisfies RMT statistics. Compared with the constantinteraction model, the Hamiltonian (159) contains a new parameter $\xi$. Its statistical properties are expected to be universal for a given value of $\xi$. An RPA estimate gives $\xi / 2 \sim 0.3 \Delta$ for GaAs dots with $r_{s} \sim 1-2$.

A quantity that might be sensitive to spin is the peak spacing. In the absence of interactions, Eq. (148) for the peak spacing still holds, but the explicit expressions for $\Delta E^{(\mathcal{N}+1)}$ and $\Delta E_{\mathcal{N}}$ depend on the parity of $\mathcal{N}: \Delta E^{(\mathcal{N}+1)}=E_{\mathcal{N} / 2+1}-E_{\mathcal{N} / 2}$ for $\mathcal{N}$ even and $\Delta E^{(\mathcal{N}+1)}=0$ for $\mathcal{N}$ odd as in the CI+SDRMT model. $\Delta E_{\mathcal{N}}$ can still be approximated by an interaction matrix element [see Eq. (149)]. However, for even

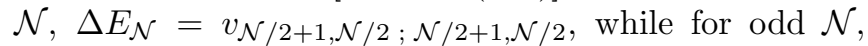
$\Delta E_{\mathcal{N}}=\int d \boldsymbol{r} d \boldsymbol{r}^{\prime}\left|\psi_{(\mathcal{N}+1) / 2}(\boldsymbol{r})\right|^{2} v\left(\boldsymbol{r}, \boldsymbol{r}^{\prime}\right)\left|\psi_{(\mathcal{N}+1) / 2}\left(\boldsymbol{r}^{\prime}\right)\right|^{2}$, since the $\mathcal{N}+1$ electron (with spin down) is now added to the same spatial orbital occupied by a spin-up electron. Using the effective RPA interaction (151), we obtain a decomposition of $\Delta E_{\mathcal{N}}$ into three parts, as in Eq. (152). While the charging energy term and $\Delta E_{\mathcal{N}}^{(1)}$ are similar in magnitude for both odd and even $\mathcal{N}$, the term $\Delta E_{\mathcal{N}}^{(2)}$, which originates from the two-body screened interaction, is on average larger for odd $\mathcal{N}$ than it is for even $\mathcal{N}$, since in the former case the spin-up and spin-down electrons occupy the same spatial wave function. We find (Mirlin, 1997)

$$
\begin{aligned}
2 \tilde{\xi} & \left.\equiv \overline{\Delta E_{\mathcal{N}}^{(2)}}\right|_{\text {odd } \mathcal{N}}-\left.\overline{\Delta E_{\mathcal{N}}^{(2)}}\right|_{\text {even } \mathcal{N}} \\
& \approx \frac{2}{\beta \mathcal{A}^{2}} \int d \boldsymbol{r} d \boldsymbol{r}^{\prime} f_{d}^{2}\left(\left|\boldsymbol{r}-\boldsymbol{r}^{\prime}\right|\right) v_{\kappa}\left(\boldsymbol{r}-\boldsymbol{r}^{\prime}\right)
\end{aligned}
$$

This shift moves the average peak spacing for odd $\mathcal{N}$ towards the average spacing for even $\mathcal{N}$ and is expected to reduce the degree of bimodality. In the RPA regime, the estimate for this shift, $\sim \Delta\left(\kappa / k_{F}\right) \ln \left(k_{F} / \kappa\right)$, is small compared with $\Delta$, since the screening length is much larger than $\lambda_{F}\left(\kappa / k_{F} \sim r_{s} \ll 1\right)$. Thus, in the weak-interaction limit, the odd-even structure is expected to persist. However, as $r_{s}$ increases, the shift increases towards $\Delta$ and the bimodality is expected to be lost. We remark that the considerations leading to Eq. (160) are based on $S=0$ and $S=1 / 2$ ground states of the dot with even and odd $\mathcal{N}$, respectively. For stronger exchange interactions, we expect a spin distribution in the dot (see end of this section) that will further affect the spacings distribution.

Can signatures of spin pairing be observed in the peak-spacing distribution? In a recent experiment, Lüscher et al. (2000) used a GaAs quantum dot with a relatively high density, $n_{s}=5.9 \times 10^{15} \mathrm{~m}^{-2}$, corresponding to $r_{s}=0.72$, a value smaller than in previous experiments. While the measured peak-spacing distribution does not have a bimodal structure, it is found to be asymmetric. It is interpreted as a superposition of two components that evolve from the noninteracting formula (145): (i) The $\delta$ function in Eq. (145) is shifted by an amount $\tilde{\xi} / \Delta$ [see Eq. (160)] and broadened to a Gaussian of width $\sigma$ (due to fluctuations of the interaction matrix elements); (ii) The Wigner-Dyson distribution in Eq. (145) is shifted to have an average of $2-\tilde{\xi} / \Delta$ (since the spacing from the upper level of a spin pair to the lower level of the next spin pair is reduced from $2 \Delta$ to $2 \Delta-\tilde{\xi})$, and is convoluted with the Gaussian of width $\sigma$. Good fits are obtained with two fit parameters $\tilde{\xi}$ and $\sigma$. Of the two, $\tilde{\xi}$ is found to be smaller in the presence of magnetic field, in qualitative agreement with theory [see, for example, Eq. (160)].

To study spin effects at intermediate and strong values of $r_{s}$, Berkovits (1998) extended his exact diagonalization calculations of the Anderson model with interactions to include the spin degrees of freedom. Denoting by $a_{i s}^{\dagger}$ and $a_{i s}$ the creation and annihilation operators of an electron with spin $s= \pm 1 / 2$ at lattice site $i$, he included in the interaction part $\mathrm{H}_{2}$ of the Hamiltonian an on-site interaction between electrons with opposite spins (in addition to the long-range Coulomb interaction among electrons at different sites):

$$
\begin{aligned}
H_{2}= & \frac{1}{2} U_{c} \sum_{\substack{i \neq j \\
s, s^{\prime}}} \frac{1}{\left|\boldsymbol{r}_{i}-\boldsymbol{r}_{j}\right| / a} a_{i s}^{\dagger} a_{i s} a_{j s^{\prime}}^{\dagger} a_{j s^{\prime}} \\
& +\frac{1}{2} U^{\prime} \sum_{i, s} a_{i s}^{\dagger} a_{i s} a_{i-s}^{\dagger} a_{i-s} .
\end{aligned}
$$

The on-site interaction $U^{\prime}=10 U_{c} / 3$ was chosen to agree with Hubbard's estimate based on hydrogenlike orbitals (Hubbard, 1963). Calculations were done for up to $\mathcal{N}=$ 9 electrons on a $3 \times 4$ lattice. The standard deviation of $\Delta_{2}$ is shown in Fig. 25(a) as a function of $U_{c}$ and for both an odd and an even number of electrons. The oddeven asymmetry of $\sigma\left(\Delta_{2}\right)$, expected in the noninteracting limit, disappears above $U_{c} \sim 0.6\left(r_{s} \sim 0.3\right)$. The peakspacing distributions are shown in Fig. 25(b) for several 

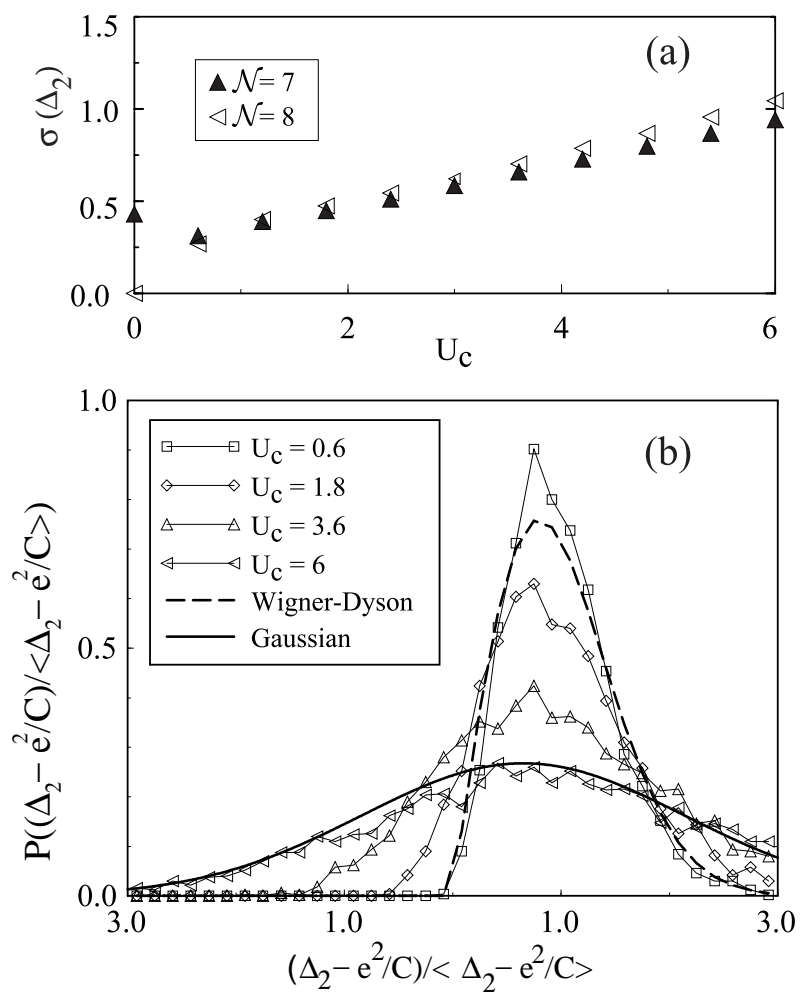

FIG. 25. Peak-spacing statistics in the Anderson model with $w / t=3$ and Coulomb interactions [Eq. (161)]: (a) the standard deviation $\sigma\left(\Delta_{2}\right)$ (in units of $\Delta$ ) as a function of the Coulomb interaction strength $U_{c}$ for $\mathcal{N}=7$ and 8 electrons in the dot; (b) the distributions of $\left(\Delta_{2}-e^{2} / C\right) /\left\langle\Delta_{2}-e^{2} / C\right\rangle$ for several values of $U_{c}$ (note that $\left\langle\Delta_{2}-e^{2} / C\right\rangle \approx 0.81 \Delta$ ). The dashed line is the Wigner-Dyson distribution and the solid line is a Gaussian fit to the $U_{c}=6$ distribution. Notice the absence of a bimodal distribution already at weak interactions and the crossover to Gaussian shapes at stronger interactions. From Berkovits (1998).

values of the interaction parameter $U_{c}$. For $U_{c}=0$ (not shown) the distribution is bimodal [see Eq. (145)], as expected. However, already for $U_{c}=0.6\left(r_{s} \sim 0.3\right)$ this bimodal structure is lost. The calculated distributions for weak interactions $\left(U_{c} \sim 0.5-2\right)$ are closer to the usual spinless constant-interaction-plus-RMT model (dashed line) rather than to the CI+SRRMT model (not shown). For $r_{s} \gtrsim 1$, the distribution is approximately a Gaussian with a width that continues to increase with $r_{s}$.

Another signature of spin and interactions is expected when a conductance peak position is followed as a function of an external parameter. Baranger, Ullmo, and Glazman (2000) suggested the appearance of kinks, i.e., abrupt changes in the parametric dependence of a Coulomb-blockade peak position. This is illustrated in Fig. 26. Consider for simplicity an even number of electrons. In the absence of interactions, the single-particle levels are doubly degenerate and follow a Gaussian process as a function of the parameter $x$. Because of interactions, the top two electrons can either occupy the
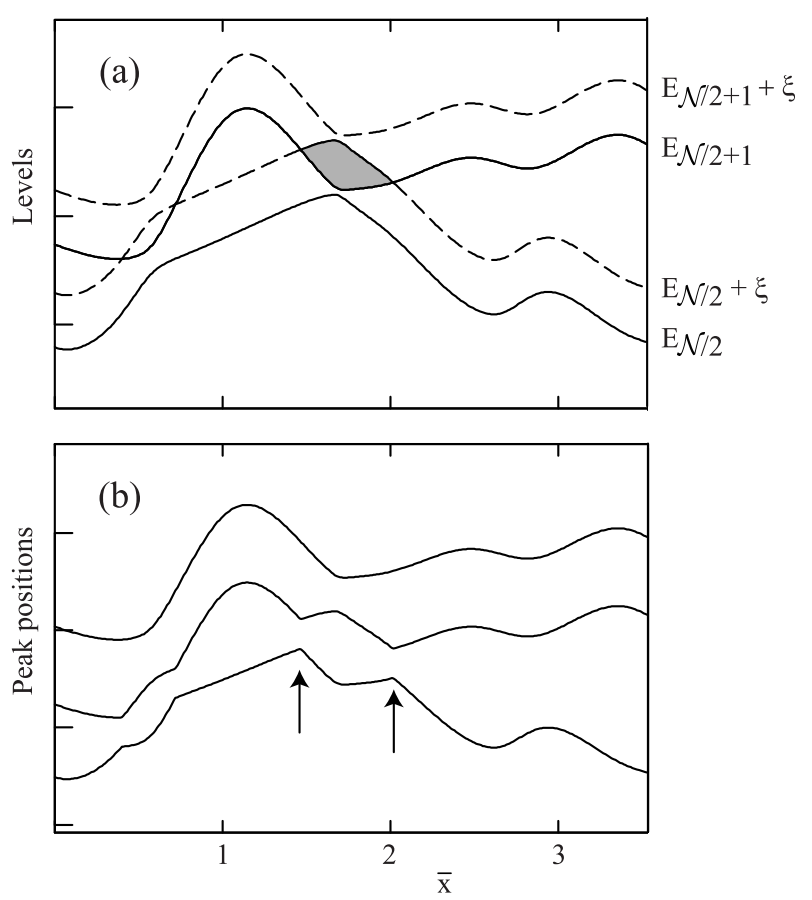

FIG. 26. Spin pairing leading to kinks in the Coulombblockade peak positions: (a) the solid lines are two orbital levels $E_{\mathcal{N} / 2}$ and $E_{\mathcal{N} / 2+1}$ from a Gaussian process vs a scaled parameter $\bar{x}$ (assuming even $\mathcal{N}$ ). When a second electron with opposite spin occupies such a level, its energy is displaced by an interaction energy $\bar{\xi}$. The displaced levels are shown by dashed lines. In the vicinity of the avoided crossing shown by the shaded area, the triplet state (top two electrons occupy levels $E_{\mathcal{N} / 2}$ and $E_{\mathcal{N} / 2+1}$ ) has a lower energy than the singlet state (the electrons occupy levels $E_{\mathcal{N} / 2}$ and $E_{\mathcal{N} / 2}+\bar{\xi}$ ). (b) Coulomb-blockade peak positions corresponding to the level diagram in panel (a) (traces are offset by the charging energy). Crossings of the singlet and triplet states lead to kinks in the peak position. A pair of such kinks in the vicinity of an avoided crossing is indicated by arrows. From Baranger, Ullmo, and Glazman (2000).

same spatial orbital, forming a singlet $S=0$ at the cost of an average interaction energy $\xi$, or fill two successive orbitals, forming a triplet $S=1$ at the cost of a kinetic energy $E_{\mathcal{N} / 2+1}-E_{\mathcal{N} / 2}$ [see Eq. (159)]. Suppose that $S=0$; we can imagine the top two electrons filling paired levels $E_{\mathcal{N} / 2}$ and $E_{\mathcal{N} / 2}+\xi$. As we change the parameter $x$, the next single-particle level $E_{\mathcal{N} / 2+1}$ can intersect $E_{\mathcal{N} / 2}+\xi$, in which case the triplet $S=1$ becomes the lowest state and the top two electrons will occupy the levels $E_{\mathcal{N} / 2}$ and $E_{\mathcal{N} / 2+1}$ [see, for example, the shaded region in Fig. 26(a)]. This configuration change causes a kink in the peak position [see Fig. 26(b)]. Kinks are more likely to occur near an avoided crossing of the levels and therefore appear in pairs versus the parameter $x$. We note that a kink occurs in the limit when only the ground-state level participates in the conductance. However, around a kink both the singlet and the triplet levels contribute to the conductance, so that the kinks in Fig. 26(b) are 
expected to become smoother. Recently Lüscher et al. (2000) observed apparent signatures of kinks versus a magnetic field $B$. Spin-paired levels are identified by the correlation of their magnetoconductance traces. These correlations are interrupted at certain values of $B$ and an apparent kink in the peak position is observed, presumably due to a rearrangement of the ground-state spin. Baranger, Ullmo, and Glazman (2000) showed that the average density of the kinks and the distribution of their separation are sensitive to the breaking of time-reversal symmetry by a magnetic field.

Another important question is the ground-state spin distribution in a disordered or chaotic dot. The exchange interaction favors a spin-polarized state, while the kinetic energy is minimized when the single-particle orbits are occupied pairwise, leading to an unpolarized $S=0$ state (for an even number of electrons). In a clean metal, the (unscreened) short-range part of the Coulomb interaction leads to a ferromagnetic (spin-polarized) instability when $\xi / 2 \gtrsim \Delta$. This is known as the Stoner instability. Andreev and Kamenev (1998) studied the Stoner instability in a dot or metallic nanoparticle in the presence of disorder. They took into account only the disorder-averaged diagonal matrix elements of the interaction $\bar{v}_{\alpha \beta ; \alpha \beta}$ and $\bar{v}_{\alpha \beta ; \beta \alpha}$ and found that the Stoner instability can develop even though the clean system is still paramagnetic. This can be traced to the effective enhancement of the interaction by the diffusive dynamics where the electrons spend more time together (Altshuler and Aronov, 1985). Brouwer, Oreg, and Halperin (1999) considered a onebody RMT Hamiltonian with on-site Hubbard interaction and computed the spin distribution of the ground state in the mean-field approximation. They concluded that the probability of a nonzero spin state can be appreciable even for interaction strengths below the Stoner instability. Jacquod and Stone (2000) pointed out that fluctuations of the off-diagonal interaction matrix elements (in the disorder basis) favor minimal spin for the ground state and therefore compete with exchange effects that favor large spin at strong interactions. The fluctuations of the interaction matrix elements determine the bandwidth of the many-body density of states and are largest for minimal spin. This effect is demonstrated using a random interaction matrix model (see Sec. VI.D). A similar model for nuclei also showed high probability of a zero-spin ground state in even-even nuclei (Johnson, Bertsch, and Dean, 1998)

Berkovits (1998) studied the ground-state spin in exact simulations of the small Anderson model plus interactions [Eq. (161)]. For $r_{s}<1$ there is a finite probability for $S=1$ states for an even number of electrons but almost no $S=3 / 2$ states for an odd number of electrons. Indeed, a spin flip costs a kinetic energy of $E_{\mathcal{N} / 2+1}-E_{\mathcal{N} / 2}$ for even $\mathcal{N}$ but $E_{(\mathcal{N}+3) / 2}-E_{(\mathcal{N}-1) / 2}$ for odd $\mathcal{N}$, and it is much less likely to find two consecutive small single-particle level spacings than one small spacing. For $r_{s}>1$, higher spin values can occur.

Experimentally, the spin of a quantum dot is difficult to measure. A promising technique is conductance measurements in the presence of an in-plane magnetic field that leads to a Zeeman splitting of the ground state. For a theoretical study of the (in-plane) magnetic field dependence of the conductance peak position and height, see Kurland, Berkovits, and Altshuler (2000). Nonzero spin has been observed in metallic nanoparticles (Ralph, Black, and Tinkham, 1997; Davidović and Tinkham, 1999) and in some carbon nanotube ropes (Cobden et al., 1998).

\section{Peak-height statistics and interactions}

How do interactions affect the distributions of the conductance peak heights and their parametric correlations? Experimentally, the predictions of RMT were confirmed (Chang et al., 1996; Folk et al., 1996) for the distributions and for the parametric correlations. However, a semiclassical estimate of the correlation flux gives $\Phi_{c} \sim 0.3 \Phi_{0}$ (assuming a geometric factor of order unity) - significantly lower than the experimental value of $\Phi \sim 0.8 \Phi_{0}$ (see Sec. V.C). Since the geometrical factor is unknown, we cannot completely rule out a single-particle theory, but the discrepancy suggests that the interactions affect the correlation flux. Here $\Phi_{c}$ is a nonuniversal parameter that depends on the details of the system's dynamics and cannot be calculated in a pure RMT.

Berkovits and Sivan (1998) used the spinless Anderson model with Coulomb interactions to study numerically the peak-height statistics and sensitivity to a magnetic flux. A $4 \times 6$ lattice with disorder $w=3 t$ was used for $\mathcal{N}=3$ and $\mathcal{N}=4$ electrons.

At low temperatures, the conductance peak height is still given by Eq. (24), but $\Gamma^{l(r)}$ are partial widths of the many-particle ground state of the dot. $R$-matrix theory (Sec. II.C) is also valid for a many-body system (Lane and Thomas, 1958). The partial-width amplitude is expressed as in Eq. (17) but with $\psi_{\lambda}$ replaced by the interacting many-body eigenfunction $\Psi_{\mathcal{N}}$ of $\mathcal{N}$ electrons in the dot, and the channel wave function $\Phi$ describing the transverse wave function of one electron in the lead and the interacting wave function $\Psi_{\mathcal{N}-1}$ of $\mathcal{N}-1$ electrons in the dot. In second-quantized notation (Berkovits and Sivan, 1998)

$$
\Gamma^{l(r)} \propto\left|\sum_{m \in l(r)}\left\langle\Psi_{\mathcal{N}+1}\left|a_{m}^{\dagger}\right| \Psi_{\mathcal{N}}\right\rangle\right|^{2}
$$

where $a_{m}^{\dagger}$ is the creation operator of the electron injected into the dot at the site $m$ belonging to the respective lead.

The width average and variance are found to decrease with $r_{s}$. This suppression is explained by an interaction- 
induced short-range order. The distributions of the dimensionless peak heights are shown in Figs. 27(a) and 27(b) for several values of the interaction strength $U_{c}$.
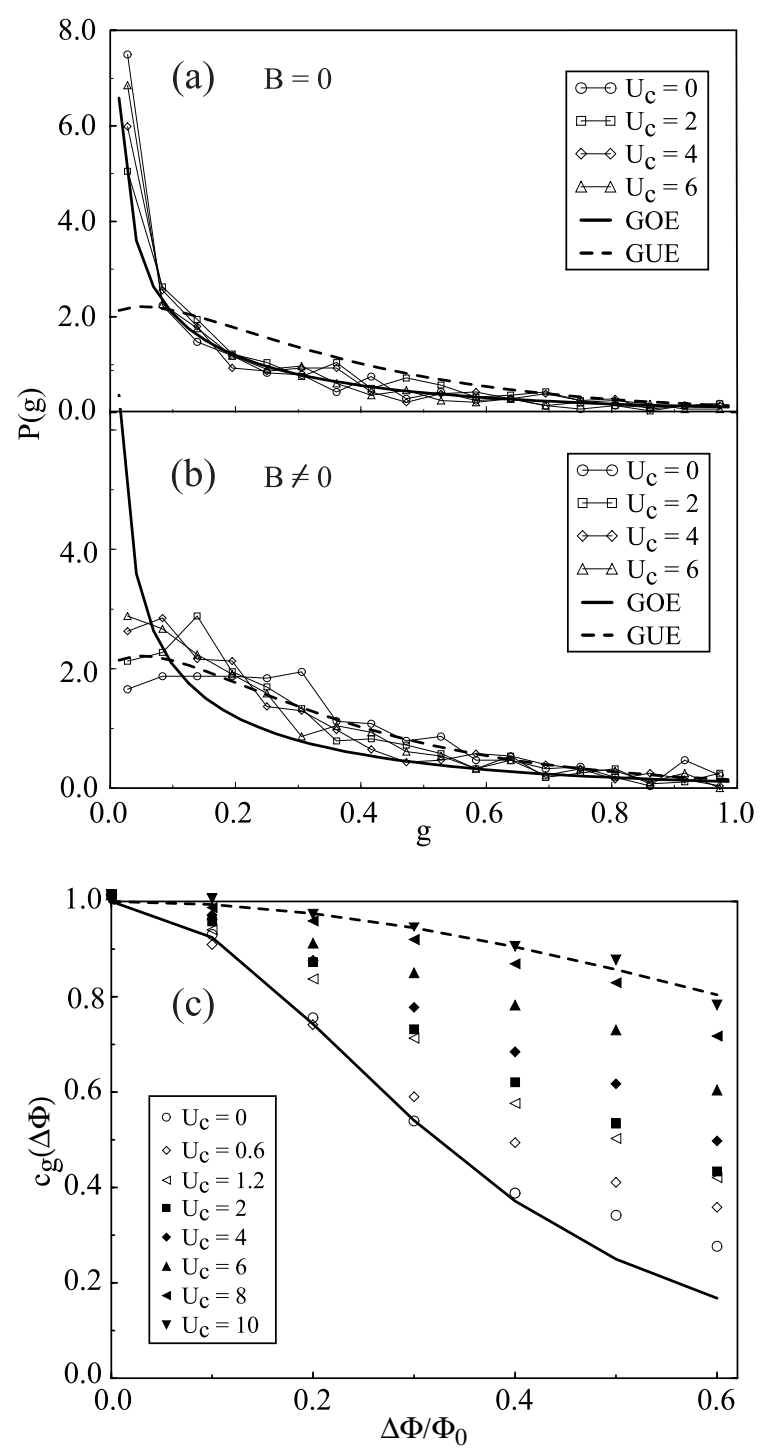

FIG. 27. Interaction effects on the conductance peak height statistics and parametric correlations: Panels (a) and (b), conductance peak-height distributions in the presence of Coulomb interactions. The distributions $P(g)$ [where $g$ is defined in Eq. (24b)] are calculated from simulations of a 2D Anderson model with Coulomb interactions. Results are shown for several values of the interaction parameter $U_{c}=0,2,4,6$ (a) without and (b) with a magnetic field $B$; solid lines, GOE prediction [Eq. (128a)]; dashed lines, GUE prediction [Eq. (128b)]. The distributions show only weak sensitivity to the strength of the interaction and are in agreement with the RMT predictions. (c) The autocorrelation function $c_{g}(\Delta \Phi)$ of the conductance peak height vs magnetic flux $\Delta \Phi$ for several values of $U_{c}$. The correlators at $U_{c}=0$ and $U_{c}=10$ can be fitted to the RMT form [Eq. (133)], but with $\Phi_{c}=0.5 \Phi_{0}$ (solid line) and $\Phi_{c}=1.75 \Phi_{0}$ (dashed line), respectively. Note the strong sensitivity of the correlation flux to the interaction strength. From Berkovits and Sivan (1998).
They are rather insensitive to the two-body interaction, confirming the predictions of RMT for both conserved $(\Phi=0)$ and broken $\left(\Phi=0.4 \Phi_{0}\right)$ time-reversal symmetry. At large values of $r_{s}$, some enhancement of the small conductance probability is observed in the case of $\Phi \neq 0$, in agreement with the experimental results of Chang et al. (1996). Similar conclusions are reached when spin is included in the model as in Sec. VI.B (Berkovits, 1999).

The parametric correlator of the peak height $G$ as a function of magnetic flux, $c_{g}(\Delta \Phi) \equiv$ $\overline{\delta G(\Phi) \delta G(\Phi+\Delta \Phi)} /\left\{\overline{[\delta G(\Phi)]^{2}} \overline{[\delta G(\Phi+\Delta \Phi)]^{2}}\right\}^{1 / 2}$, where $\delta G=G-\bar{G}$, is shown in Fig. 27(c) for different values of $U_{c}$. At $r_{s}=0$ and $r_{s} \gtrsim 1.4$, the RMT functional form, Eq. (80), of the correlator is reproduced. However, the value of the correlation flux is increased from its noninteracting value $\Phi_{c}=0.5 \Phi_{0}$ to $\Phi_{c}=1.75 \Phi_{0}$ at $r_{s} \sim 3.5$. Thus, in the regime relevant to semiconductor quantum dots, the correlation field is a factor of $2-3$ larger than its noninteracting value, in general agreement with the experiment.

\section{Random interaction matrix model}

An important issue is whether RMT can be used to describe interacting systems. RMT was originally developed to describe the statistics of a strongly interacting system - the compound nucleus - at high excitations. Calculations in interacting systems in nuclear, atomic, and condensed-matter physics suggest that the WignerDyson statistics are generic to complex many-body systems at sufficiently large excitations (Montambaux et al., 1993; Flambaum et al., 1994; Zelevinsky et al., 1996). In quantum dots, however, our interest is in the statistical behavior at or near the system's ground state as the number of electrons varies. Standard RMT makes no explicit reference to interactions or number of particles. Generic interaction effects on the statistics can be studied in a random-matrix model that contains interactions explicitly. A two-body random-interaction matrix model, introduced in nuclear physics by French and Wong (1970) and Bohigas and Flores (1971), was used together with a random single-particle spectrum to study thermalization (Flambaum, Gribakin, and Izrailev, 1996) and the transition from Poisson to Wigner-Dyson statistics (Jacquod and Shepelyansky, 1997) in manybody systems. However, the Poissonian single-particle statistics of this model are not suitable for studying dots whose single-particle dynamics is chaotic. Alhassid, Jacquod, and Wobst (2000) introduced a random interaction matrix model (RIMM) to study generic fluctuations in chaotic dots with interactions. The RIMM is an ensemble of interacting Hamiltonians, 


$$
H=\sum_{i j} h_{i j} a_{i}^{\dagger} a_{j}+\frac{1}{4} \sum_{i j k l} u_{i j k l}^{A} a_{i}^{\dagger} a_{j}^{\dagger} a_{l} a_{k}
$$

where the one-body matrix elements $h_{i j}$ are chosen from the appropriate Gaussian random-matrix ensemble, and the antisymmetrized two-body matrix elements $u_{i j ; k l}^{A} \equiv$ $u_{i j ; k l}-u_{i j ; l k}$ form a GOE in the two-particle space

$$
P(h) \propto e^{-\frac{\beta}{2 a^{2}} \operatorname{Tr} h^{2}} ; \quad P\left(u^{A}\right) \propto e^{-\operatorname{Tr}\left(u^{A}\right)^{2} / 2 U^{2}} .
$$

The variance of the diagonal (off-diagonal) interaction matrix elements is $U^{2}\left(U^{2} / 2\right)$. The states $|i\rangle=a_{i}^{\dagger}|0\rangle$ describe a fixed basis of $m$ single-particle states. Here $h$ is an $m \times m$ GOE (GUE) matrix when the single-particle dynamics conserve (break) time-reversal symmetry, while the two-body interaction preserves time-reversal symmetry and forms a GOE, irrespective of the symmetry of the one-body Hamiltonian. In general, the two-body interaction can include a nonvanishing average part $\bar{u}$ that is invariant under orthogonal transformations of the singleparticle basis. For spinless electrons, the only such invariant is the charging energy $e^{2} \mathcal{N}^{2} / 2 C$, which is a constant and does not affect the statistical fluctuations of (163). In the presence of spin, an additional contribution to $\bar{u}$ is an exchange interaction $-\xi \boldsymbol{S}^{2} / 2$. We remark that in a physical model of a dot, the Coulomb interaction matrix elements are given in a fixed basis. Fluctuations of the interaction matrix elements were introduced in the RIMM to obtain a generic model that is independent of a particular interaction, in the original spirit of RMT (French and Wong, 1970; Bohigas and Flores, 1971).

The model was used to study both the peak-spacing (Alhassid, Jacquod, and Wobst, 2000) and peak-height statistics (Alhassid and Wobst, 2000). The peak-spacing distribution describes a crossover from a Wigner-Dyson distribution to a Gaussian-like distribution as $U / \Delta$ increases [see Fig. 28(a)]. The partial level width is calculated from an expression analogous to Eq. (162), and for a GOE one-body Hamiltonian, its distribution $P(\hat{\Gamma})$ is found to be a GOE Porter-Thomas distribution independent of $U / \Delta$. However, for a GUE one-body Hamiltonian, the width distribution makes a crossover from GUE to a GOE Porter-Thomas distribution as a function of $U / \Delta$ [see Fig. 28(b)]. The crossover distributions are well described by $P_{\zeta}(\Gamma)$ of Eq. (72), where $\zeta$ is a monotonically decreasing function of $U / \Delta$. This is due to the competition between the asymptotic GUE symmetry of the one-body Hamiltonian $h$ and the GOE symmetry of the two-body interaction $u$. In the range $U / \Delta \sim 0.7-1.5$, the peak-spacing distribution is already Gaussian-like, while the width statistics are still close to the GUE limit. In the RIMM, $U / \Delta$ is a free parameter, and reasonable values can be determined by comparing its results against physical models. Such a comparison was made to a small $(4 \times 5)$ Anderson model with Coulomb interactions and periodic boundary conditions. Apart from finite-size effects, similar behavior was observed where the range $U / \Delta \sim 0.7-1.5$ in the RIMM corresponded to $U_{c} / t \sim 2-5$ in the Coulomb model.
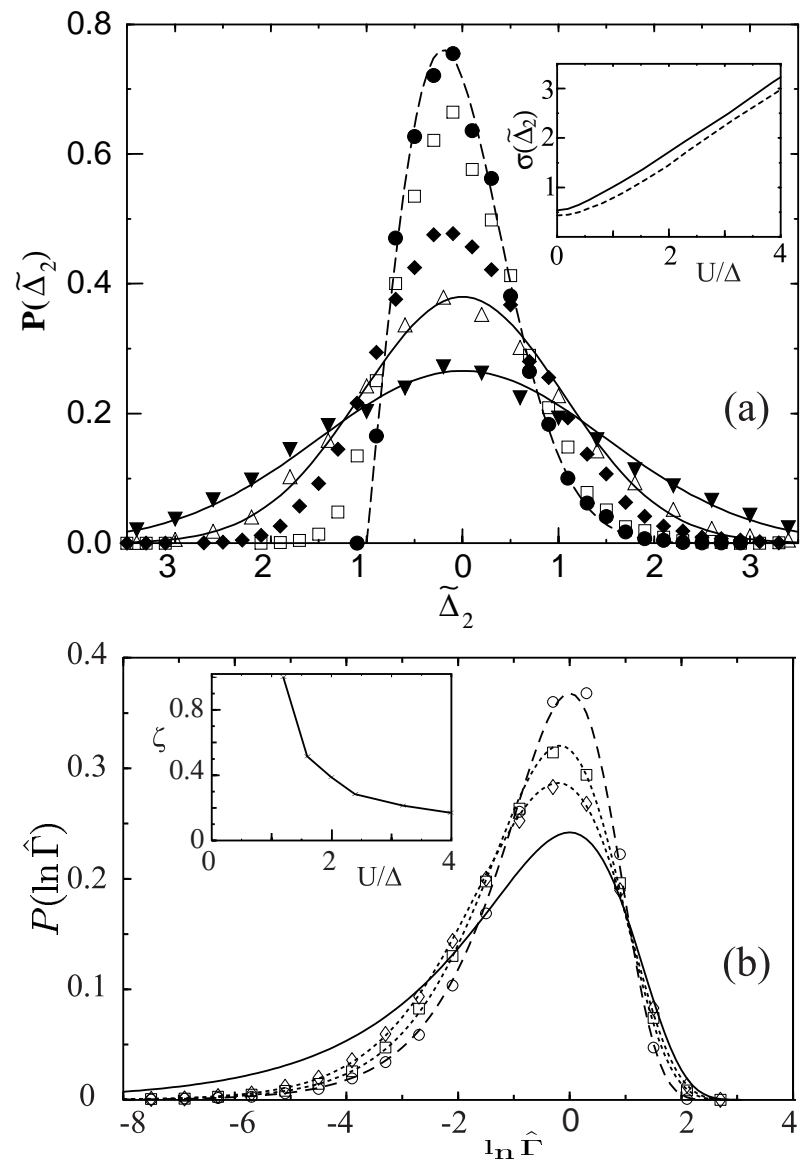

FIG. 28. Peak-spacing and peak-height statistics in the random interaction matrix model [RIMM; Eq.(163)]. (a) Peakspacing distributions $P\left(\tilde{\Delta}_{2}\right)$ in the RIMM with GOE onebody statistics for $m=12, \mathcal{N}=4$ : solid circles, $U / \Delta=0$; open squares, $U / \Delta=0.35$; solid diamonds, $U / \Delta=0.7$; open triangles, $U / \Delta=1.1$; solid triangles, $U / \Delta=1.8$. Notice the crossover from a Wigner-Dyson distribution at $U=0$ (dashed line) to Gaussian-like distributions (solid lines) for $U / \Delta \gtrsim 1$. Inset to panel (a), standard deviation $\sigma\left(\tilde{\Delta}_{2}\right)$ of the peak spacings vs $U / \Delta$ for GOE (solid) and GUE (dashed) one-body statistics. (b) Width distributions in the RIMM with GUE one-body statistics. Distributions $P(\ln \hat{\Gamma})$ vs $\ln \hat{\Gamma}$ are shown for $m=12, \mathcal{N}=4$ : open circles, $U / \Delta=0$; open squares, $U / \Delta=2.4$; open diamonds, $U / \Delta=4$; solid line, GOE Porter-Thomas distribution; dashed line, GUE PorterThomas distribution; short-dashed lines; analytic width distributions $P_{\zeta}(\ln \hat{\Gamma})$ in the crossover between the GUE and GOE (see Section V.D.1). Inset to panel (b), crossover parameter $\zeta$ vs $U / \Delta$. Adapted from Alhassid, Jacquod and Wobst (2000) and Alhassid and Wobst (2000). 


\section{CHARGING ENERGY EFFECTS IN QUANTUM DOTS}

In this section we discuss other mesoscopic phenomena in quantum dots where the charging energy plays an important role: fluctuations of the off-resonance conductance in closed (or semiopen) dots and mesoscopic Coulomb blockade in dots that are strongly coupled to a single-channel lead.

The dominant mechanism for the off-resonance conductance is cotunneling (see Sec. II.F). In particular, for $k T<\sqrt{E_{C} \Delta}$, the dominant process is elastic cotunneling, describing the virtual tunneling of an electron or hole through a large number $\sim E_{C} / \Delta$ of excited levels in the dot. The coherent superposition of a large number of weak amplitudes exhibits fluctuation properties that are qualitatively different from those of the conductance peaks. Since a virtual transition occurs across a gap of $\sim E_{C} / 2$, we expect the charging energy to play an important role in determining the statistics of the minima. The mesoscopic fluctuations in elastic cotunneling were derived by Aleiner and Glazman (1996) and observed by Cronenwett et al. (1997). They are discussed in Sec. VII.A.

In the crossover from closed to open dots, the classical Coulomb-blockade oscillations (observed in the limit $\Delta \rightarrow 0$ ) gradually weaken. Matveev (1995) showed that these oscillations completely disappear for a fully transmitting one-channel lead $\left(T_{c}=1\right)$. However, Aleiner and Glazman (1998) showed that for a dot with finite $\Delta$, quantum Coulomb blockade is not fully destroyed and the conductance exhibits mesoscopic fluctuations that are periodic in the gate voltage but have a random phase. The signatures of this mesoscopic Coulomb blockade can be seen in the correlation functions of various fluctuating observables. The main mechanism for these fluctuations is the backscattering of electrons from the boundaries of the dot into the strongly coupled channel. An experiment by Cronenwett, et al. (1998) confirms signatures of mesoscopic Coulomb blockade in dots with one fully open lead $T^{l} \sim 1$ and one weakly coupled lead $T^{r} \ll 1$. Among the striking effects seen is the strong suppression of Coulomb-blockade oscillations at finite magnetic fields, contrary to the behavior in closed dots. Mesoscopic Coulomb blockade is discussed in Sec. VII.B.

In a partially open dot, the charge is not quantized and exhibits mesoscopic fluctuations, as does the differential capacitance $d Q / d V_{g}$. The capacitance fluctuations in an open dot were derived by Gopar, Mello, and Büttiker (1996) in the limit of noninteracting electrons using the distribution of the scattering time delays in the dot. However, the charging energy should be taken into account in dots with a partially open single channel. The statistical properties of the capacitance fluctuations in the presence of charging energy were calculated by Kaminski, Aleiner, and Glazman (1998), and are discussed in Sec. VII.C.

\section{A. Mesoscopic fluctuations in elastic cotunneling}

The mesoscopic fluctuations of the conductance minima were derived by Aleiner and Glazman (1996) in the diagrammatic approach. According to Eq. (38), the off-resonance conductance amplitude is determined by a large number of fluctuating terms, of which $\sim E_{e} / \Delta$ and $\sim E_{h} / \Delta$ contribute significantly to the sum over particles and over holes, respectively. When $E_{e}$ and $E_{h}$ are below the Thouless energy, the contributing levels are in the universal regime where the partial widths $\gamma_{c \lambda}$ and energies $E_{\lambda}$ have RMT statistics. In this regime, we can apply an RMT approach for both the on- and off-resonance conductance. In both cases the mesoscopic fluctuations are determined by the same underlying statistics of the partial widths of the resonance levels. The difference between the statistics of the conductance maxima and minima originates from their different transport mechanisms.

We first discuss the distribution of the conductance minima in the crossover between conserved and broken time-reversal symmetry. The cotunneling amplitude $\mathcal{T}$ in Eq. (38) is the sum of a large number of terms, and we expect the central limit theorem to apply, leading to Gaussian distributions for both the real and the imaginary parts of $\mathcal{T}$. The distribution of $G \propto(\operatorname{Re} \mathcal{T})^{2}+(\operatorname{Im} \mathcal{T})^{2}$ (measured in units of $\bar{G}$ ) is then given by

$$
P(\hat{G})=(1-\chi)^{-1} e^{-\frac{\hat{G}}{1-\chi}} I_{0}\left(\frac{\sqrt{\chi}}{1-\chi} \hat{G}\right),
$$

where $I_{0}$ is a Bessel function and $\chi \equiv\left\{\overline{(\operatorname{Re} \mathcal{T})^{2}} / \overline{|\mathcal{T}|^{2}}-\overline{(\operatorname{Im} \mathcal{T})^{2}} / \overline{|\mathcal{T}|^{2}}\right\}^{2}$. An expression of the form (165) was obtained by Aleiner and Glazman (1996) when the time-reversal symmetry was broken by a magnetic field $B$. They found $\chi=\Lambda\left(B / B_{c}^{\text {valley }}\right)$, where

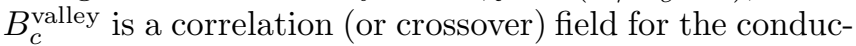
tance "valleys" [see Eq. (168) below] and

$$
\begin{aligned}
\Lambda(x) \equiv & \frac{1}{\pi^{2} x^{4}}\left[\ln x^{2} \ln \left(1+x^{4}\right)+\pi \arctan x^{2}\right. \\
& \left.+\operatorname{Li}_{2}\left(-x^{4}\right) / 2\right]^{2} .
\end{aligned}
$$

The function $\mathrm{Li}_{2}$ in Eq. (166) is the second polylogarithm function.

The average cotunneling conductance is found to be

$$
\bar{G}=\frac{e^{2}}{h}\left(\frac{\overline{\Gamma^{l}}}{\Delta} \frac{\overline{\Gamma^{r}}}{\Delta}\right) \Delta\left(\frac{1}{E_{h}}+\frac{1}{E_{e}}\right),
$$

where $E_{e}\left(E_{h}\right)$ is the distance between the Fermi energy and the closest state available for electron (hole) tunneling (see Sec. II.F). Thus, in striking contrast to the 
average conductance peak, the average off-resonance conductance is independent of magnetic field and does not exhibit a weak-localization effect. This has been confirmed experimentally (Cronenwett et al., 1997).

The correlation field $B_{c}^{\text {valley }}$ for the conductance valleys (which is of the same order as the crossover field) can be estimated semiclassically as for the conductance peaks, except that the Heisenberg time $\tau_{H}=h / \Delta$ is now replaced by the virtual tunneling time $h / E$, where $E=\min \left(E_{e}, E_{h}\right)$. In analogy with Eq. (133) we find

$$
B_{c}^{\text {valley }} \mathcal{A} / \Phi_{0}=\kappa \sqrt{E / E_{T}},
$$

where $E_{T} \sim \hbar v_{F} / \sqrt{\mathcal{A}}$ is the ballistic Thouless energy, and $\kappa$ is a geometric factor of the dot. The valley correlation field is seen to be larger than the peak correlation field

$$
B_{c}^{\text {valley }} / B_{c}^{\text {peak }}=\sqrt{E / \Delta} .
$$

We next turn to the parametric correlations of the cotunneling conductance versus magnetic field (Aleiner and Glazman, 1996). Assuming a point-contact model, the cotunneling amplitude [Eq. (38)] can be rewritten as

$$
\begin{aligned}
\mathcal{T}= & \frac{\sqrt{\bar{\Gamma}^{l} \bar{\Gamma}^{r}}}{\Delta} \frac{1}{\nu} \int \frac{d \omega}{2 \pi i}\left[G^{A}\left(\boldsymbol{r}_{l}, \boldsymbol{r}_{r}, \omega\right)\right. \\
& \left.-G^{R}\left(\boldsymbol{r}_{l}, \boldsymbol{r}_{r}, \omega\right)\right] G^{\mathrm{ret}}(\omega) .
\end{aligned}
$$

The quantity $\nu$ is the average density of states in the dot per unit area, $G^{R}$ and $G^{A}$ are retarded and advanced Green's functions of the non-interacting dot and $G^{\text {ret }}(\omega)=-\left(|\omega|+E_{e}\right)^{-1}+\left(|\omega|+E_{h}\right)^{-1}$ is the retarded cotunneling Green's function of a dot with interactions (Baltin and Gefen, 2000). The calculation of the parametric correlator requires the ensemble averages of the corresponding products of Green's functions. In the metallic regime and for $E \gg \Delta$, the latter can be calculated in the diagrammatic approach and expressed in terms of the diffuson $\mathcal{D}$ and the cooperon $\mathcal{C}$ : $\overline{G_{B}^{R}\left(\boldsymbol{r}, \boldsymbol{r}^{\prime}, \omega\right) G_{B^{\prime}}^{A}\left(\boldsymbol{r}^{\prime}, \boldsymbol{r}, \omega^{\prime}\right)}=2 \pi \nu \mathcal{D}_{B, B^{\prime}}\left(\boldsymbol{r}, \boldsymbol{r}^{\prime}, \omega-\omega^{\prime}\right)$ and $\overline{G_{B}^{R}\left(\boldsymbol{r}, \boldsymbol{r}^{\prime}, \omega\right) G_{B^{\prime}}^{A}\left(\boldsymbol{r}, \boldsymbol{r}^{\prime}, \omega^{\prime}\right)}=2 \pi \nu \mathcal{C}_{B, B^{\prime}}\left(\boldsymbol{r}, \boldsymbol{r}^{\prime}, \omega-\omega^{\prime}\right)$. If $E<E_{T}$, a typical $\Delta \omega=\omega-\omega^{\prime}$ that contributes to the correlator is below $E_{T}$ and one can use the zero-mode approximation:

$$
\begin{aligned}
\mathcal{D}_{B, B^{\prime}} & =\frac{1}{\mathcal{A}}\left[-i \Delta \omega+\left(\mathcal{A} \frac{B-B^{\prime}}{\Phi_{0}}\right)^{2} E_{T}\right]^{-1} \\
\mathcal{C}_{B, B^{\prime}} & =\frac{1}{\mathcal{A}}\left[-i \Delta \omega+\left(\mathcal{A} \frac{B+B^{\prime}}{\Phi_{0}}\right)^{2} E_{T}\right]^{-1}
\end{aligned}
$$

Equations (171) lead to a universal parametric correlator for the off-resonance conductance

$$
\begin{aligned}
c_{G}(\Delta B) & =\overline{\delta G(B) \delta G\left(B^{\prime}\right)} / \bar{G}^{2} \\
& =\Lambda\left(\frac{\Delta B}{B_{c}^{\text {valley }}}\right)+\Lambda\left(\frac{2 B+\Delta B}{B_{c}^{\text {valley }}}\right),
\end{aligned}
$$

where $\Lambda(x)$ is the scaling function (166) and the correlation field $B_{c}^{\text {valley }}$ is given by Eq. (168), with $\kappa=1$. The GUE correlator is obtained from Eq. (172) in the limit $B \gg B_{c}^{\text {valley }}$, where $c_{G}(\Delta B)=\Lambda\left(\Delta B / B_{c}^{\text {valley }}\right)$. (a)

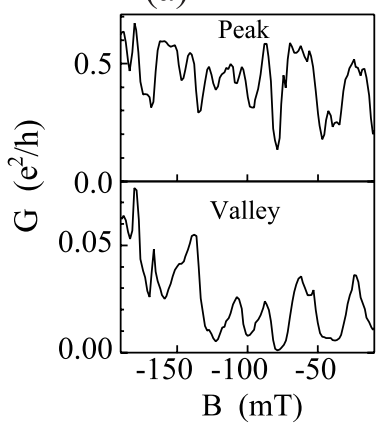

(c)

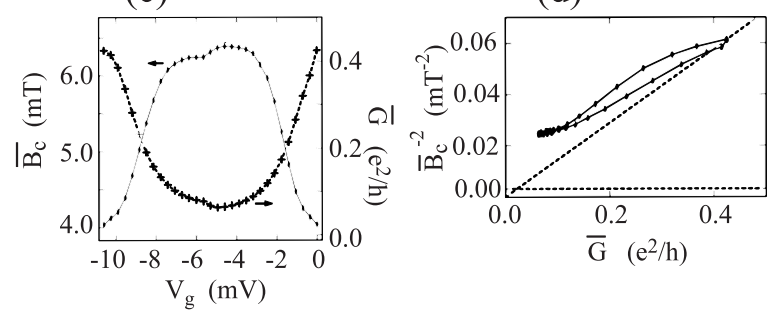

FIG. 29. Elastic cotunneling in Coulomb-blockade dots: panels (a) and (b), conductance fluctuations of a peak vs a valley. Panel (a), a conductance peak and an adjacent conductance minimum (valley) as a function of a magnetic field $B$ (different scales are used for $G$ at a peak or a valley). The valley fluctuates on a longer scale than the peak. Panel (b), the measured conductance correlator vs $\Delta B$ for a peak (dashed line) and for a valley (solid line). Panels (c) and (d) describe an experimental test of the scaling relation (173). Panel (c), the measured average correlation field $\bar{B}_{c}$ (solid line) and the average conductance $\bar{G}$ (dashed line) as the gate voltage varies between two neighboring peaks. The ensemble-averaged $\bar{B}_{c}$ is obtained from $\sim 30$ statistically independent traces of the conductance. Panel (d), $\bar{B}_{c}^{-2}$ as a function of $\bar{G}$ (solid line) using the same data as in (c). The diagonal dashed line is Eq. (173). From Cronenwett et al. (1997) and Marcus et al. (1997).

The correlation field $B_{c}^{\text {valley }}$ for the conductance valleys is enhanced by $\sqrt{E / \Delta}$ compared with the correlation field of the peaks [see Eq. (169)]. This enhancement was observed in the experimental results of Cronenwett et al. (1997) shown in Fig. 29. In order to measure the weak conductance in the valleys, the dots used were semiopen with $\bar{\Gamma} \sim 0.7 \Delta$. Typical fluctuations versus magnetic field of a conductance peak in comparison with a conductance valley are shown in Fig. 29(a). The valley fluctuates on a scale that is larger than the scale over which a peak fluctuates. The conductance autocorrelation functions for the peaks and the valleys are shown in Fig. 29(b). The observed ratio $B_{c}^{\text {valley }} / B_{c}^{\text {peak }} \sim 1.6$ is smaller than the expected value of $\sim 4$. The discrepancy 
is not fully understood, but we remark that (i) the peak correlation field is larger than the single-particle estimate owing to interaction effects (see Sec. VI.C), and (ii) the theoretical estimate for the peak correlation field is for $T \ll \Delta$, while in the experiment $T \sim \Delta$. Both effects lead to enhancement of the peak correlation field and might explain the observed reduction in the valley-to-peak ratio of the correlation fields.

The enhancement of the correlation field for the offresonance conductance is a charging energy effect. According to (168), the largest correlation field is obtained at midvalley, where $E$ obtains its maximal value $E_{C} / 2$. At this gate voltage the average conductance is minimal according to Eq. (167). Using the approximation $\bar{G} \propto E^{-1}$ [see Eq. (167)], we obtain

$$
B_{c}^{2} \bar{G} \approx \text { const. }
$$

Thus the correlation field is maximal when the average conductance is smallest, and vice versa. Figure 29 (c) shows the measured correlation field $B_{c}$ (solid line), averaged over independent peak-valley-peak data sets, and the average conductance $\bar{G}$ (dashed line) plotted as a function of gate voltage in the region between two Coulomb-blockade peaks. To test the approximate scaling relation (173), the same data are used to plot $\bar{B}_{c}^{-2}$ versus $\bar{G}$ in Fig. 29(d) (solid lines). The diagonal dashed line is the scaling relation (173).

Baltin and Gefen (2000) have used Eq. (170) to calculate the normalized cotunneling conductance correlator between valleys $\mathcal{N}$ and $\mathcal{N}+n$. Parametrizing the fractional distance between two neighboring peaks by $y$ [so that $E_{h}=y E_{C}$ and $\left.E_{e}=(1-y) E_{C}\right]$, they find

$$
\begin{aligned}
c_{G}(n, y)= & {\left[\frac{y(1-y)}{\left(1+n \frac{\Delta}{E_{C}}\right) n \frac{\Delta}{E_{C}}}\right]^{2}\left[\ln \left(1+\frac{n}{y} \frac{\Delta}{E_{C}}\right)\right.} \\
& \left.+\ln \left(1+\frac{n}{1-y} \frac{\Delta}{E_{C}}\right)\right]^{2} .
\end{aligned}
$$

The cotunneling amplitudes in two neighboring valleys contain a sum over similar contributions from intermediate states, except that one particle state becomes a hole state. The correlator (174) is then expected to decay slowly with $n$ on a scale set by $\sim E_{C} / \Delta$. The measured correlator (Cronenwett et al., 1997) is found to decay faster, presumably due to scrambling of the singleparticle spectrum when an electron is added to the dot.

Another interesting issue is the phase change $\Delta \alpha$ of the transmission amplitude between two consecutive valleys. Two experiments (Yacoby et al., 1995; Schuster et al., 1997) have measured the phase of the transmission amplitude through a dot, employing an interferometer with two arms, one of which contains a quantum dot. A surprising result of these experiments was that $\Delta \alpha=0(\bmod 2 \pi)$ across all measured peaks. Since the phase changes by $\pi$ across a resonance, an additional "phase lapse" of $\pi$ must occur. This is contrary to what one expects in a dot with noninteracting electrons where the values of $\Delta \alpha$ across a series of peaks form some sequence of 0 and $\pi$. Various possible explanations have assumed specific geometries that lead to preferred levels in the dot (see, for example, Baltin et al., 1999). A more generic mechanism for a disordered or chaotic dot, suggested by Baltin and Gefen (1999), employs a formula that interpolates between the valley transmission amplitude [Eq. (38)] and the BreitWigner peak amplitude (see also Oreg and Gefen, 1997). An approximate sign sum rule states that the number of $\pi$ changes of the phase between two neighboring valleys (each due to a resonance, a near-resonance phase lapse, or a valley phase lapse) is even. The probability to deviate from this sign sum rule is small $\left(\sim \Delta / E_{C}\right)$. The sign sum rule relies on the strong correlations of the transmission phase in neighboring valleys, and spectral scrambling would lead to its breakdown. However, in the experiments only $\Delta \alpha=0$ was observed.

At very low temperatures and in the strong-tunneling regime, the valley conductance that corresponds to an odd number of electrons can be enhanced due to the Kondo effect. Higher-order virtual tunneling processes that effectively flip the unpaired spin on the dot can lead to a coherent many-body resonance at the Fermi energy, known as the Kondo resonance (Glazman and Raikh, 1988; Ng and Lee, 1988; Meir, Wingreen, and Lee, 1993). It is formed between the spin of the dot and the delocalized electrons in the leads - in analogy to the Kondo effect (Kondo, 1964), which occurs when a magnetic impurity is placed in a metal and the unpaired electron in the impurity forms a singlet state with the metal's electrons. The Kondo effect in a quantum dot was only recently observed by Goldhaber-Gordon et al. (1998) and by Cronenwett, Oosterkamp, and Kouwenhoven (1998). The energy scale for observing the Kondo resonance is the Kondo temperature $T_{K}$, which is essentially the binding energy of the resonance. At midvalley $T_{K} \sim \sqrt{E_{C} \Gamma} e^{-\pi E_{C} / 8 \Gamma}$, and to bring $T_{K}$ within the range of experimentally accessible temperatures it was necessary to fabricate much smaller dots $(L \sim 100 \mathrm{~nm})$. In a smaller dot, $\Delta$ is larger and $\Gamma$ can be increased substantially while the dot remains semiopen (i.e., $\Gamma<\Delta$ ). The appearance of a Kondo resonance in the density of states enhances the conductance in the valley that corresponds to an odd number of electrons as the temperature decreases below $T_{K}$.

\section{B. Mesoscopic Coulomb blockade}

In Secs. IV and V we discussed mesoscopic fluctuations of the conductance in two opposite limits - open dots and almost-closed dots. In the crossover from closed to 
open dots, the charge quantization and the Coulombblockade oscillations gradually disappear. An interesting case is a lead with one fully transmitting channel. Rather than discussing a dot with symmetric leads, it is easier to study the asymmetric limit where, e.g., the left lead has a transmission coefficient of $T^{l} \approx 1$, while the right lead has a large barrier $\left(T^{r} \ll 1\right)$. The Hamiltonian of this system is different from the Hamiltonian (36) used to describe cotunneling in that the subsystem of the dot plus the left lead is now described by a single Hamiltonian (because of the strong coupling):

$$
\begin{aligned}
H= & \int d \boldsymbol{r} \psi^{\dagger}(\boldsymbol{r})\left[-\nabla^{2} / 2 m^{*}+U(\boldsymbol{r})-\mu\right] \psi(\boldsymbol{r})+\frac{\hat{Q}^{2}}{2 C} \\
& -\alpha V_{g} \hat{Q}+\sum_{k, s \in r} E_{k s} c_{k s}^{\dagger} c_{k s} \\
& +\sum_{k, s \in r}\left[V_{k s} c_{k s}^{\dagger} \psi\left(\boldsymbol{r}_{\boldsymbol{c}}\right)+H . c .\right]
\end{aligned}
$$

The first term on the right describes the dot-channel Hamiltonian with a confining potential $U(\boldsymbol{r})$ and chemical potential $\mu\left[\psi^{\dagger}(\boldsymbol{r})\right.$ is the creation operator of an electron at $\boldsymbol{r}]$. The term $\hat{Q}^{2} / 2 C$, with $\hat{Q}=e \hat{\mathcal{N}} \equiv$ $e \int_{\text {dot }} d \boldsymbol{r} \psi^{\dagger}(r) \psi(\boldsymbol{r})$ being the charge operator of the dot, describes the Coulomb interaction in the constantinteraction model. The last two terms describe the Hamiltonian of the right lead and the tunneling Hamiltonian between the dot and the right lead (where $c_{k s}^{\dagger}$ is the creation operator of an electron in the leads with wave number $k$ and spin $s$, and $\boldsymbol{r}_{c}$ is the right point contact).

Furusaki and Matveev (1995a, 1995b) calculated the conductance for the Hamiltonian (175) in the classical Coulomb-blockade limit $k T \gg \Delta$. The calculation is nonperturbative in the charging energy. In the limit of perfect transmission $\left(T^{l}=1\right)$, the dot-channel Hamiltonian can be effectively described by a one-dimensional fermionic Hamiltonian that is solved using bosonization methods. At perfect transmission, Coulomb blockade disappears, but even a small reflection (due to a weak backscatterer at the dot-channel interface) causes Coulomb-blockade oscillations with an amplitude that depends quadratically on temperature away from the charge degeneracy points, in analogy with inelastic cotunneling.

Aleiner and Glazman (1998) generalized the model to the quantum regime where the temperature is comparable to $\Delta$ and found mesoscopic Coulomb-blockade effects that resemble elastic cotunneling. The coherent backscattering of an electron into the lead mimics the backscatterer effects in the classical case and leads to mesoscopic Coulomb-blockade oscillations even at perfect transmission $\left(T^{l}=1\right)$. However, their phase is random, and the signature of mesoscopic Coulomb blockade is thus best quantified in terms of conductance correlations at different values of the gate voltage.
Since the dot is in $2 \mathrm{D}$, it is not possible to describe the backscattering from the dot's boundaries by a onedimensional Hamiltonian. Instead, an effective action that is nonlocal in time but expressed in terms of the onedimensional channel variables is derived. The charging energy is then treated exactly using bosonization methods. For spinless electrons and in the limit $T^{l}=1$, the correlation function of the conductance fluctuations versus gate voltage is given by

$$
\begin{aligned}
& \overline{\delta G\left(V_{g 1}\right) \delta G\left(V_{g 2}\right)} / G^{r 2} \\
& \quad=0.78 \beta^{-1}\left(\frac{\Delta}{E_{C}}\right)^{2} \cos \left(2 \pi \frac{\alpha C}{e} \Delta V_{g}\right),
\end{aligned}
$$

where $G^{r}$ is the conductance of the right point contact. The average conductance and its standard deviation are proportional to $\Delta / E_{C}$, similarly to elastic cotunneling. The periodicity of the correlation function versus gate voltage corresponds to a period of one electron charge and is just the manifestation of mesoscopic Coulomb blockade. The fluctuations are larger in the absence of magnetic field $(\beta=1)$, since the constructive interference of time-reversed trajectories enhances coherent backscattering.

When the spin of the electrons is taken into account, the results are quite different:

$$
\begin{aligned}
& \overline{\delta G\left(V_{g 1}\right) \delta G\left(V_{g 2}\right)} / G^{r 2} \\
& =0.83 \beta^{-2} \frac{\Delta}{T}\left(\frac{\Delta}{E_{C}}\right)^{2} \ln ^{3}\left(\frac{E_{C}}{T}\right) \cos \left(2 \pi \frac{\alpha C}{e} \Delta V_{g}\right) .
\end{aligned}
$$

In particular, the mesoscopic fluctuations become sensitive to temperature, and the suppression of the fluctuations with magnetic field is stronger.

Some of the predicted characteristics of mesoscopic Coulomb blockade were confirmed qualitatively in an experiment by Cronenwett et al. (1998). Experimentally, it is more convenient to study the power spectrum $P_{G}(f)$ of $G\left(V_{g}\right)$, i.e., the Fourier transform of the correlation function (177). It is found to be centered in a narrow band around the Coulomb-blockade frequency, and the integrated power is given by

$$
P(T)=0.207 G^{r 2} \beta^{-2} \frac{\Delta}{T}\left(\frac{\Delta}{E_{C}}\right)^{2} \ln ^{3}\left(\frac{E_{C}}{k T}\right) .
$$

The crossover field from conserved to broken timereversal symmetry is similar to the one found for the conductance minima. For magnetic fields that are large compared with this correlation field $(\beta=2)$, the power of the mesoscopic fluctuations is expected to be four times smaller than without a magnetic field $(\beta=1)$. Figure 30 compares typical Coulomb-blockade fluctuations as a function of gate voltage for the one-channel regime (left) and the weak-tunneling regime (right), both with [panels (c) and (d)] and without [panels (a) and (b)] a magnetic 
field. A striking effect is the strong suppression of the fluctuations in the one-channel regime when a magnetic field is applied. This strong sensitivity to magnetic field is also seen in the integrated power spectrum versus magnetic field [Figs. 30(e) and 30(f)]. The power spectrum for the one-channel regime is strongly peaked at $B=0$, while it does not show any particular field dependence in the weak-tunneling regime. The enhancement of the $B=0$ power relative to its large- $B$ value is found to be $\sim 5.3 \pm 0.5$, somewhat larger than the predicted value of 4 . The experiment also confirms the sensitivity of the mesoscopic fluctuations to temperature.
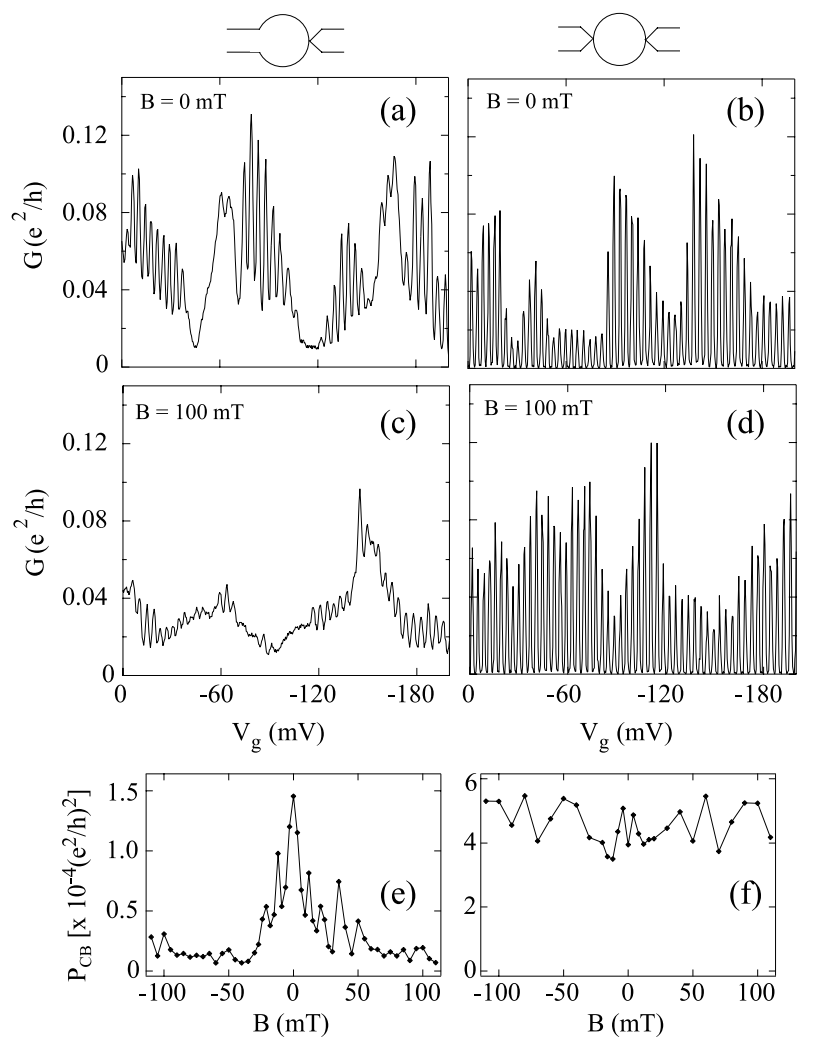

FIG. 30. Conductance fluctuations in a one-channel dot (left) and in a closed dot (right). Traces of conductance vs gate voltage are shown for [(a) and (b)] $B=0$ and [(c) and (d)] $B=100 \mathrm{mT}$. Notice that the mesoscopic Coulomb-blockade oscillations in the one-channel dot are suppressed by a magnetic field, in contrast to the closed dot. Panels (e) and (f) show the integrated power of the Coulomb-blockade oscillations as a function of magnetic field. For a one-channel dot [panel (e)] the power decreases with magnetic field, while for a closed dot [panel (f)] there is only weak dependence of the power on magnetic field. From Cronenwett et al. (1998).

Brouwer and Aleiner (1999) showed that mesoscopic charge quantization also affects the conductance in open dots with ideal leads. A charging energy term in the Hamiltonian of an open dot plus leads does not affect the average conductance in the unitary case, but does enhance the weak localization correction in the or- thogonal case $\Lambda /(4 \Lambda+2)$ [see Eq. (98a) for $\beta=1$ ] by $\left(c_{\Lambda} / \Lambda\right) \Lambda \Delta /\left(8 \pi^{2} T\right)$, where $\Lambda$ is the number of open channels in each lead $\left(c_{1} \approx 3.18\right.$ and $\left.c_{\infty}=\pi^{2} / 6\right)$. Furthermore, the variance of $G$ in the unitary case, $\Lambda \Delta /(96 T)$ [see Eq. (113)], acquires an additional term $\left(c_{\Lambda} / \Lambda\right) \Lambda^{2} \Delta^{2} /\left(32 \pi^{2} T^{2}\right)$ in the limit $T \gg \Lambda \Delta$ and with $c_{\Lambda} \approx 6.49$ for $\Lambda \gg 1$.

\section{Mesoscopic fluctuations of the differential capacitance}

In the weak-coupling regime (where the transmission coefficient $T_{c} \ll 1$ ), the charge on the dot is quantized and increases in a steplike manner as the gate voltage changes. As the conductance of the point contact increases (but is still small compared with $e^{2} / h$ ), the deviation of the average charge from its quantized value is linear in $T_{c}$ (Glazman and Matveev, 1990b; Matveev, 1991), except near the degeneracy points where the average charge increases sharply by about one unit $e$. A related quantity is the differential capacitance of the $\operatorname{dot} C_{\text {diff }}\left(V_{g}\right) \equiv \partial Q / \partial V_{\text {ext }}=\alpha^{-1} \partial Q / \partial V_{g}$, which, in the weak-coupling limit, exhibits sharp peaks at the degeneracy points of $V_{g}$. Matveev (1995) and Flensberg (1993) showed that when a single-channel lead is connected to a dot and in the classical limit $\Delta \rightarrow 0$, Coulomb blockade vanishes at perfect transmission (i.e., $T_{c}=1$ ). In this limit, the average charge increases linearly as a function of gate voltage and the differential capacitance is a constant. However, for nearly perfect transmission (i.e., $T_{c}$ slightly smaller than 1 ), the average charge and differential capacitance exhibit weak Coulomb-blockade oscillations versus gate voltage.

Aleiner and Glazman (1998) studied the mesoscopic fluctuations of the differential capacitance (of a dot with single-channel lead) for finite $\Delta$ assuming the dynamics in the dot is chaotic. In the case of perfect transmission, $\overline{\delta C_{\text {diff }}\left(V_{g}\right)}=0$, and the Coulomb-blockade periodicity is seen in the correlation function of the differential capacitance versus gate voltage. The capacitance correlations are found to be

$$
\begin{aligned}
& \overline{\delta C_{\text {diff }}\left(V_{g 1}\right) \delta C_{\text {diff }}\left(V_{g 2}\right)} / C^{2} \\
& =5.59 \beta^{-1}\left(\frac{\Delta}{E_{C}}\right) \cos \left(2 \pi \frac{\alpha C}{e} \Delta V_{g}\right) \\
& \overline{\delta C_{\text {diff }}\left(V_{g 1}\right) \delta C_{\text {diff }}\left(V_{g 2}\right)} / C^{2} \\
& =0.54 \beta^{-1}\left(\frac{\Delta}{E_{C}}\right) \ln ^{3}\left(\frac{E_{C}}{T}\right)\left[\left(\frac{\Delta}{E_{C}}\right) \ln \left(\frac{E_{C}}{T}\right)\right] \\
& \quad \times \cos \left(2 \pi \frac{\alpha C}{e} \Delta V_{g}\right)
\end{aligned}
$$

for the cases without and with spin, respectively.

Capacitance fluctuations in the weak-coupling regime were studied by Kaminski, Aleiner and Glazman (1998). 
Away from the peaks, the capacitance could be calculated by treating the tunneling Hamiltonian as a perturbation. The capacitance fluctuations could then be related to the fluctuations of the single-electron Green's function of the dot at the point contact. The latter are universal when $E=\min \left(E_{e}, E_{h}\right)$ (see Sec. II.F) is below the Thouless energy and could be expressed in terms of the diffuson and cooperon [Eqs. (171)]. For example, the standard deviation of the capacitance fluctuations in the absence of magnetic field was found to be $\sigma\left(\delta C_{\text {diff }}\right)=$ $\left(C g_{0} / \sqrt{6} \pi^{2}\right)(\Delta / E)^{1 / 2}\left(E_{C} / E\right)$, where $g_{0}=G_{0} /\left(2 e^{2} / h\right)$ $(\ll 1)$ is the dimensionless point-contact conductance. The correlation field of the capacitance fluctuations in a magnetic field had the same scale as the correlation field [Eq. (168)] for the cotunneling conductance fluctuations. The capacitance fluctuations were also studied in the strong-tunneling regime, and it was concluded that the maximal fluctuations are reached for a partially open channel $\left(G_{0}<2 e^{2} / h\right)$.

Recently, high-sensitivity single-electron transistors were used to measure a dot's charge and capacitance, and fluctuations of the differential capacitance were observed (Berman et al., 1999). However, the accuracy of these experiments is insufficient to quantify these fluctuations. Kaminski and Glazman (1999) pointed out that certain mesoscopic fluctuations in a partially open dot can be more easily measured in a double-dot geometry, where each dot is weakly coupled to a lead. The interdot coupling can be adjusted, resulting in peak doublets as a function of the gate voltages on each of the dots. The spacing between the doublets fluctuates because of the fluctuations of the interdot tunneling amplitude. The rms-to-average ratio of the doublet spacing was found to be $\sim\left(2 \Delta / \beta E_{C}\right)^{1 / 2}$. This provides information on the mesoscopic fluctuations of the ground-state energy of a partially open dot, which are otherwise difficult to measure directly.

\section{CONCLUSION AND FUTURE DIRECTIONS}

Since they were first produced about a decade ago, quantum dots have become a powerful tool for investigating the physics of small, coherent quantum systems. The ability to control their shape, size, number of electrons, and coupling strength has made them particularly attractive for experimental studies. This review has focused on the statistical regime of quantum dots, a regime characterized by quantum interference effects, chaotic dynamics of the quasiparticles, and electron-electron interaction effects.

Table I summarizes the main mesoscopic effects in quantum dots versus the main theoretical techniques used to calculate them. For each case, a reference is made to the equation (in parentheses) and/or the figure

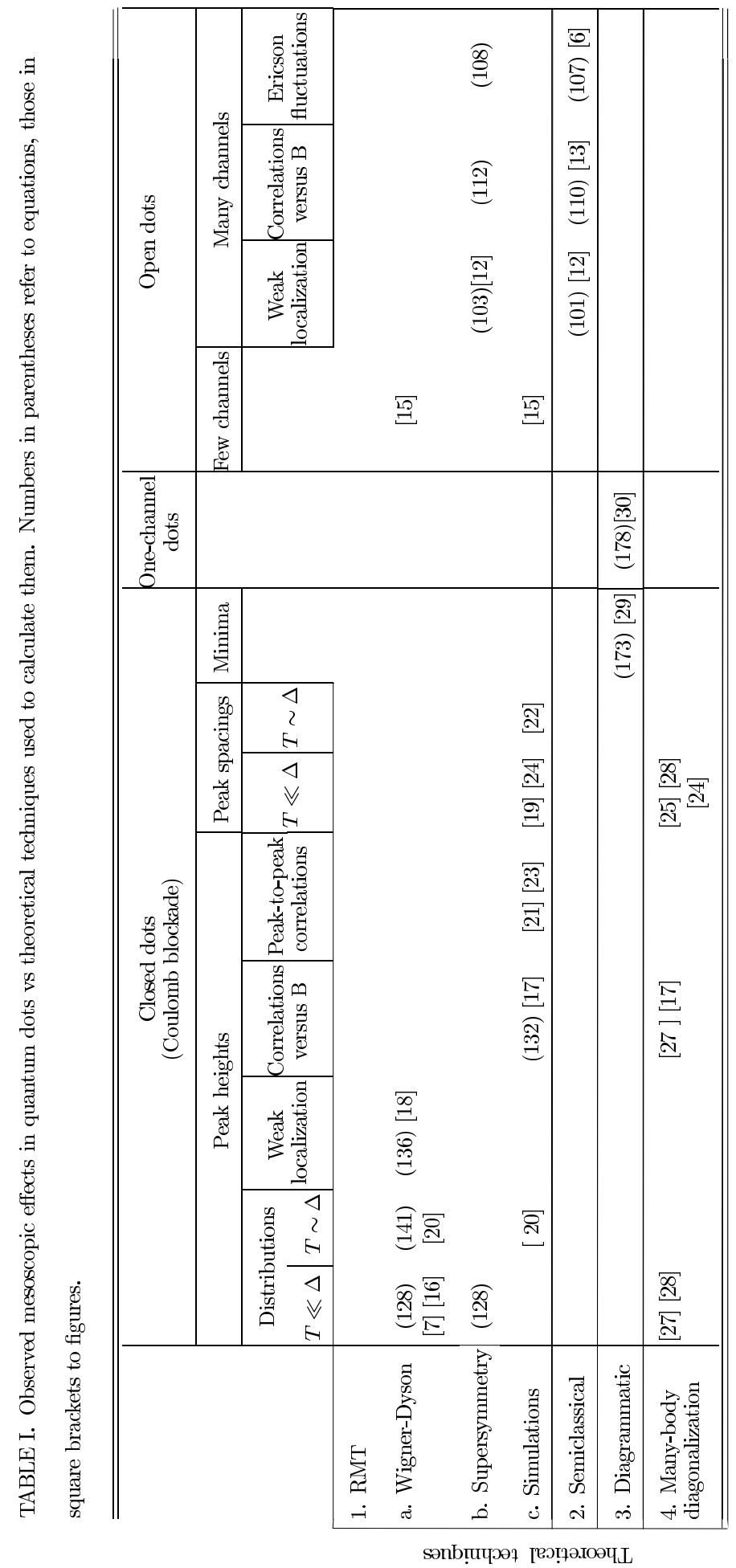

(in square brackets) that is relevant to the effect. The table is restricted to effects that have been experimentally observed.

Quantum dots have several energy scales. Among them are the mean level spacing $\Delta$, arising from the confinement of the electrons and inversely proportional to 
the dot's area, and the average level width $\bar{\Gamma}$, representing the strength of the dot-lead couplings. In an open dot, where $\bar{\Gamma} \gg \Delta$, the electrons can be treated as noninteracting quasiparticles, and electron-electron interactions are considered indirectly through their effect on the decoherence rate $\Gamma_{\phi}$. The limit $\Gamma_{\phi} \ll \bar{\Gamma}$ (i.e., $\tau_{\text {escape }} \ll \tau_{\phi}$ ) is the limit of full phase coherence, where quantum interference effects dominate the mesoscopic fluctuations of the conductance. The universality of these fluctuations is determined by another energy scale $E_{T}$ - the ballistic Thouless energy (or the Thouless energy in a disordered dot). For $\bar{\Gamma} \ll E_{T}$ (i.e., $\tau_{\text {escape }} \gg \tau_{c}$, where $\tau_{c}$ is the ergodic time) the fluctuations are universal. Finite temperature can reduce the fluctuations through thermal smearing and shorter dephasing times. In the absence of dephasing, the fluctuations are largest and temperature independent when $T \ll \bar{\Gamma}$.

Phase breaking becomes important at temperatures where $\Gamma_{\phi}$ is comparable to $\bar{\Gamma}$ and leads to deviations from universality. Indeed, even at the lowest temperatures attained in the experiments, dephasing must be included to obtain good agreement between theory and experiment.

With the introduction of tunnel barriers between the dot and the leads, the charge on the dot is quantized and an additional energy scale becomes relevant - the charging energy $e^{2} / C$. In the almost-isolated dots typically used in experiments, all three scales, $\Gamma, \Delta$, and $e^{2} / C$, separate: $\bar{\Gamma} \ll \Delta \ll e^{2} / C$, and the temperature determines which energy scales are resolved. The regime of interest in this review is the quantum regime, where the temperature is around or smaller than $\Delta$ and mesoscopic fluctuations are observed. In typical experiments in closed dots, $\bar{\Gamma} \ll T$, and the level width is not directly resolved.

The statistical theory of disordered or ballistic chaotic dots is well understood in the limit of noninteracting quasiparticles. In closed dots this "single-particle" approach includes a constant-interaction term $\mathcal{N}^{2} e^{2} / 2 C$ (the constant-interaction model). While the constantinteraction model is the simplest way to include charging energy effects in closed dots, several experiments indicate that electron-electron interactions play an important role in the statistical properties of such dots. This is not surprising considering that the Coulomb interaction is rather strong in semiconductor dots where the gas parameter $r_{s}$ is $\sim 1-2$. Understanding the effects of interactions on the statistical fluctuations is one of the major current directions in mesoscopic physics in general, and in the statistical theory of quantum dots in particular. Recent progress includes Hartree-Fock and RPA estimates, but the experimental values of $r_{s}$ are in the range where it is necessary to go beyond the RPA. Currently, most of the results for $r_{s} \gtrsim 1$ are based on numerical simulations of small disordered systems that include interactions. The number of electrons used in the simulations is smaller than in the experiments. The effects seen are believed to be independent of the number of electrons, but this is not proven. Except for $r_{s} \ll 1$, it is not known what is the parametric dependence of the fluctuations on the gas parameter $r_{s}$ and on properties of the dot such as its Thouless conductance $g_{T}$.

Another problem of current interest is the role played by electron spin in closed dots. Indirect evidence of spin pairing was recently found in the statistical properties of the conductance peaks (Lüscher et al., 2000). In a chaotic or disordered dot we expect a spin distribution in the ground state, but its dependence on interactions and on the Thouless conductance $g_{T}$ is not yet understood (the limit $g_{T} \rightarrow \infty$ was recently studied by Kurland, Berkovits, and Altshuler, 2000). Hund's rules are not expected to hold in chaotic dots that do not possess any particular symmetries, and we would like to understand how the total spin of these dots changes with the addition of electrons. Several experimental groups are now working on measuring spin in dots. A promising technique is an in-plane magnetic field that couples to the spin but does not directly affect the orbital motion.

While the limits of almost-closed $\operatorname{dots}(\bar{\Gamma} \ll \Delta)$ and open dots $(\bar{\Gamma} \gg \Delta)$ have been studied extensively, less is known about the intermediate regime where the coupling to the leads is strong but some charge quantization remains. In this regime, transport becomes more complicated even within the constant-interaction model, leading to cotunneling and mesoscopic Coulomb blockade. A recent experiment (Maurer et al., 1999) investigated Coulomb-blockade fluctuations in dots with symmetric leads as a function of the dot-lead couplings. Few theoretical results are available for this intermediate regime. Some of the experimental results were not expected within the theory of cotunneling, e.g., a Kondolike anomalous temperature dependence of the conductance valleys. In general, the coupling to the leads makes interaction effects within the dot more difficult to handle than in completely closed systems. For example, at low temperatures Kondo-type resonance can be formed between an unpaired electron in the dot and the delocalized electrons in the leads, and a perturbative approach is not possible. A consistent formulation of transport in the presence of interactions can be done in the Keldysh formalism (Meir and Wingreen, 1992) but is difficult to implement.

Another important topic for future research is the statistical properties of excited states in quantum dots. Thus far, statistical studies have focused on the linear regime of a small source-drain voltage $V_{s d}$, where the observed Coulomb-blockade peaks probe the ground state of a dot with different numbers of electrons. Information about excited states in a dot with a fixed number of electrons can be obtained through nonlinear measurements (see Sec. V.H). The experiment of Stewart et al. 
(1997) suggests certain similarities between the low excitation spectrum and the addition spectrum of a quantum dot. However, the statistical properties of these low-lying states and their manifestation in the fluctuations of the nonlinear differential conductance have not been investigated.

A theory of the quasiparticle lifetime (due to electronelectron interactions) in a quantum dot has suggested that, above a critical value of the excitation energy, the quasiparticle width becomes finite (Altshuler et al., 1997). This behavior is deduced by relating the problem to Anderson localization in real space and is the result of a delocalization phase transition in Fock space where the interacting wave function becomes fragmented over a large number of noninteracting states. The crossover from Poisson to Wigner statistics at high excitations as a function of the interaction strength has been linked to this Fock-space delocalization (Berkovits and Avishai, 1998). Numerical investigations in a more realistic random-matrix model with one-body disorder and interactions (Mejia-Monasterio et al., 1998) found that at the low excitations relevant to the experiments there was a smooth crossover from almost-localized to delocalized states but no Anderson-like localization. This model could explain the main features of an experiment by Sivan et al. (1994) that found only a few $(\sim 7)$ resolved excited levels (of the order of $g_{T}$ ) before their width grew beyond the mean level spacing. Recent numerical studies suggest a possible localization transition for very large values of $g_{T}$, where the transition occurs at very high excitations (Leyronas, Tworzydlo, and Beenakker, 1999).

Several models have been proposed to explain the effect of dephasing on the conductance statistics in open dots. Dephasing in almost-closed dots and how it might affect the conductance is not well understood. Theoretical estimates for the temperature dependence of the dephasing time in closed dots due to $e-e$ interactions were derived by Sivan, Imry, and Aronov (1994) and by Blanter (1996). Full phase coherence is expected in a closed dot below a temperature that is parametrically larger than $\Delta$ (Altshuler et al., 1997). However, reduced values of the weaklocalization correction are found experimentally at lower temperatures (Folk et al., 2000). Also, deviations of the experimental peak-height distributions from the theoretical phase-coherent predictions are found to increase with temperature (Sec. V.F.1). These results suggest apparent phase breaking and are not understood. It is possible that $e$-e interaction is not the main dephasing mechanism in the experimentally studied devices. Other suggested mechanisms are external radiation (Gershenson, 1999) and nuclear spins (Dyugaev, Vagner, and Wyder, 2000).

Most investigations of transport in quantum dots have concentrated on chaotic dots. Much less is known about fluctuations in nonchaotic dots, especially in the Coulomb-blockade regime. In such systems the fluctu- ations are not expected to be universal and the semiclassical approach is the most suitable one. However, this approach encounters difficulties at long time scales of the order of the Heisenberg time. In a recent study of conductance fluctuations in an integrable cavity, the conductance fluctuations were found to increase with incoming energy (Pichaureau and Jalabert, 1999), unlike the universal fluctuations in chaotic cavities. Ketzmerick (1996) argued that the conductance through a cavity with mixed phase space displays a fractal behavior as a function of an external parameter, e.g., a magnetic field. This behavior originates from trajectories that are trapped near the boundary between regular and chaotic regions, leading to an algebraic decay of the enclosedarea distribution. The change of the conductance $\Delta G$ with a magnetic field is expected to have a variance of $\overline{(\Delta G)^{2}} \propto(\Delta B)^{\gamma}$ where $1<\gamma<2$ [in contrast with a chaotic dot where $\overline{(\Delta G)^{2}} \propto(\Delta B)^{2}$ for small $\left.\Delta B\right]$. An experiment by Sachrajda et al. (1998) claims to have observed this fractal behavior in dots coupled to unusually wide $(\sim 0.7 \mu \mathrm{m})$ leads.

In the work discussed in this review, transport through the dot is driven by an applied bias. Another way to produce a dc current through an open dot (at zero bias) is by a cyclic change of its deformation or any other parameter that affects the interference pattern of the electron's wave function. For low-frequency changes, the electrons maintain equilibrium and the device is known as an adiabatic quantum pump. In an open dot, the electronic wave function extends into the leads and an adiabatic cyclic change of at least two parameters can cause a net charge transport $Q$ per cycle. The theory of parametric pumping was worked out by Zhou, Spivak, and Altshuler (1999) and by Brouwer (1998), and an experiment was carried out by Switkes et al. (1999). While confirming some of the theoretical predictions at weak pumping (e.g., $\left\langle Q^{2}\right\rangle \propto S_{A}^{2}$ where $S_{A}$ is the area enclosed by the contour in the two-parameter space), there were unexplained quantitative differences at strong pumping (e.g., the dependence of $\left\langle Q^{2}\right\rangle$ on $S_{A}$ was slower than linear). Recently the theory was generalized to the strong-pumping regime (Shutenko, Aleiner, and Altshuler, 2000), and it was found that $\left\langle Q^{2}\right\rangle \propto l_{A}\left(l_{A}\right.$ being the length of the contour) - slower than the naive expectation of $\left\langle Q^{2}\right\rangle \propto S_{A}$. Issues of dissipation and dephasing, important for the temperature dependence of pumping, still need to be understood. Aleiner and Andreev (1998) showed that for an almost-open dot the charge transmitted in one cycle is quantized in the limit $T \rightarrow 0$.

Recent years have seen the fabrication of new conducting nanostructures smaller than quantum dots. These devices have similarities to quantum dots, and Coulombblockade peaks are observed in both linear and nonlinear $I-V$ measurements versus gate voltage. A particularly interesting example is the nanometer-scale $\mathrm{Al}$ particle, 
part of a tunneling device that includes a gate electrode (Ralph, Black, and Tinkham, 1997). The spectrum of the $\mathrm{Al}$ particle as a function of the number of electrons it contains can be determined from nonlinear measurements similar to those made on semiconductor quantum dots. Odd-even effects in the number of electrons can be understood in terms of the pairing interaction. Davidović and M. Tinkham (1999) measured the spectra of Au nanoparticles. At higher excitations the resonances of the particles overlapped to form broad resonances that eventually merged into the continuum around the Thouless energy, in overall agreement with the theory of Altshuler et al. (1997). Another type of conducting nanostructure is the carbon nanotube, which also exhibits charging-energy effects (Bockrath et al., 1997; McEuen, 1998). Figure 31 shows Coulomb-blockade peaks in the measured conductance of a nanotube, where large fluctuations of the peak heights can be seen (Cobden et al., 1998). The statistical theory of quantum dots should find interesting applications in some of these novel nanoscale devices.

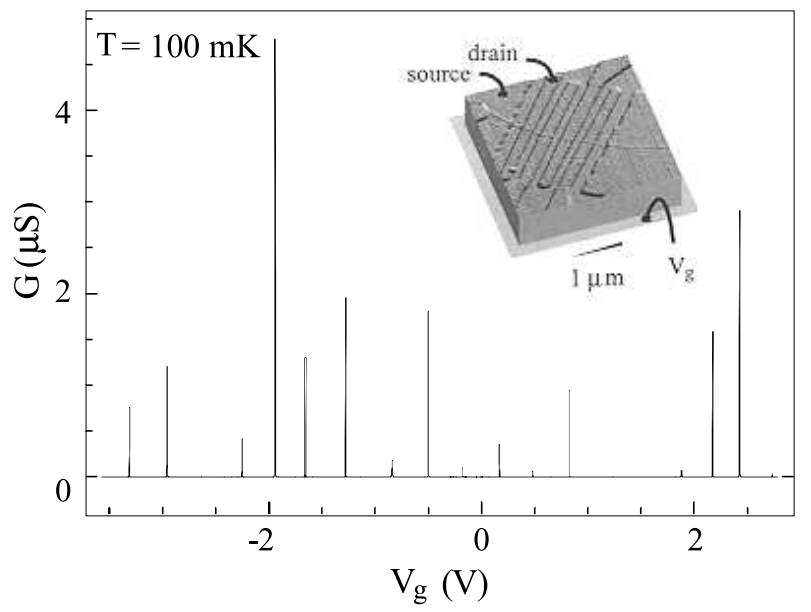

FIG. 31. The conductance vs gate voltage in a carbon nanotube rope ( $200 \mathrm{~nm}$ segment). Sharp Coulomb-blockade peaks are observed, each describing the tunneling through a single resonance level. Note the strong fluctuations in the peak heights. The inset is an image of the device with schematic wires drawn. From Cobden et al. (1998).

\section{ACKNOWLEDGMENTS}

I would like to thank H. Attias, Y. Gefen, M. Gökçedă̆, J. N. Hormuzdiar, Ph. Jacquod, R. Jalabert, S. Malhotra, S. Patel, N. Whelan and A. Wobst for fruitful collaborations on various topics in this review. Special thanks to A. D. Stone and C. M. Marcus for productive collaborations and useful discussions. I am grateful to U. Alhassid, K. Aschheim, G. F. Bertsch, C. M. Marcus, A. Polkovnikov, A. D. Stone, T. Wettig, and in particular H. A. Weidenmüller for their comments and suggestions on the manuscript. This work was supported in part by the
Department of Energy grant No. DE-FG-0291-ER-40608.

\section{REFERENCES}

Abrikosov, A.A., L.P. Gor'kov, and I.E. Dzhyaloshinskii, 1963, Methods of Quantum Field Theory in Statistical Physics (Prentice-Hall, New York).

Agam, O., B.L. Altshuler and A.V. Andreev, 1995, Phys. Rev. Lett. 75, 4389.

Ahn, K.-H., K. Richter, and I.-H. Lee, 1999, Phys. Rev. Lett. 83, 4144 .

Akkermans, E., G. Montambaux, J.-L. Pichard, and J. ZinnJustin, eds., 1995, Mesoscopic Quantum Physics (NorthHolland, Amsterdam).

Aleiner, I.L., B.L. Altshuler, and M.E. Gershenson, 1999, Waves Random Media 9, 201.

Aleiner, I.L., and A.V. Andreev, 1998, Phys. Rev. Lett. 81, 1286.

Aleiner, I.L., and L.I. Glazman, 1996, Phys. Rev. Lett. 77, 2057.

Aleiner, I.L., and L.I. Glazman, 1998, Phys. Rev. B 57, 9608. Alhassid, Y., 1998, Phys. Rev. B 58, R 13383.

Alhassid, Y., and H. Attias, 1996a, Phys. Rev. Lett. 76, 1711. Alhassid, Y., and H. Attias, 1996b, Phys. Rev. B 54, 2696.

Alhassid, Y., and H. Attias, 1995, Phys. Rev. Lett. 74, 4635.

Alhassid, Y., and Y.Gefen, 2000, "Spectral scrambling in Coulomb-blockade quantum dots," reprint.

Alhassid, Y., M. Gökçedă̆, and A.D. Stone, 1998, Phys. Rev. B 58, R7524.

Alhassid, Y., J.N. Hormuzdiar and N. Whelan, 1998, Phys. Rev. B 58, 4866.

Alhassid, Y., Ph. Jaquod, and A. Wobst, 2000, Phys. Rev. B 61, R13357.

Alhassid, Y., and R.D. Levine, 1986, Phys. Rev. Lett. 57, 2879 .

Alhassid, Y., and C.H. Lewenkopf, 1995, Phys. Rev. Lett. 75, 3922 .

Alhassid, Y., and C.H. Lewenkopf, 1997, Phys. Rev. B 55, 7749 .

Alhassid, Y., and S. Malhotra, 1999, Phys. Rev. B 60, R16315.

Alhassid, Y., and A. Wobst, 2000, "Interactioneffects on the conductance peak height statistics in quantum dots," e-print cond-mat/0003255.

Altland, A., and Y. Gefen, 1993, Phys. Rev. Lett. 71, 3339.

Altshuler, B.L., 1985, JETP Lett. 41, 648.

Altshuler, B.L., and A. G. Aronov, 1985, in Electron-Electron Interactions in Disordered Systems, edited by A.L. Efros and M. Pollak (North-Holland, Amsterdam), p. 1.

Altshuler, B.L., Y. Gefen, A. Kamenev, and L. S. Levitov, 1997, Phys. Rev. Lett. 78, 2803.

Altshuler, B.L., D.E. Khemlnitskii, A.I. Larkin, and P.A. Lee, 1980, Phys. Rev. B 20, 5142.

Altshuler, B.L., P.A. Lee, and R.A. Webb, editors, 1991, Mesoscopic Phenomena in Solids (North-Holland, Amsterdam).

Altshuler, B.L., and B.I. Shklovskii, 1986, Sov. Phys. JETP $64,127$. 
Altshuler, B.L., and B.D. Simons, 1995, in Mesoscopic Quantum Physics, edited by E. Akkermans, G. Montambaux, J.-L. Pichard, and J. Zinn-Justin (North-Holland, Amsterdam), p. 1.

Anderson, P.W., 1958, Phys. Rev. 109, 1492.

Andreev, A.V., O. Agam, B.D. Simons, and B.L. Altshuler, 1996, Phys. Rev. Lett. 76, 3947.

Andreev, A.V., and B.L. Altshuler, 1995, Phys. Rev. Lett. 75, 902 .

Andreev, A.V., and A. Kamenev, 1998, Phys. Rev. Lett. 81, 3199.

Argaman, N., 1996, Phys. Rev. B 53, 7035.

Argaman, N., J. Imry, and U. Smilansky, 1993, Phys. Rev. B 47, 4440 .

Ashoori, R.C., 1996, Nature (London) 379, 413.

Attias, H., and Y. Alhassid, 1995, Phys. Rev. E 52, 4476.

Austin, E.A., and M. Wilkinson, 1992, Nonlinearity 5, 1137.

Averin, D.V., and A.N. Korotkov, 1990, Sov. Phys. JETP 70, 937.

Averin, D.V., A.N. Korotkov, and K.K. Likharev, 1991, Phys. Rev. B 44, 6199.

Averin, D.V., and K.K. Likharev, 1986, J. Low Temp. Phys. 62, 345.

Averin, D.V., and K.K. Likharev, 1991, in Mesoscopic Phenomena in Solids, edited by B. L. Altshuler, P. A. Lee, and R. A. Webb (North-Holland, Amsterdam), p. 173.

Averin, D.V., and Yu. N. Nazarov, 1990, Phys. Rev. Lett. 65, 2446.

Balian, R., 1968, Nuovo Cimento B 57, 183.

Balian, R., and C. Bloch, 1972, Ann. Phys. (N.Y.) 69, 76.

Baltin, R., and Y. Gefen, 1999, Phys. Rev. Lett. 83, 5094.

Baltin, R., and Y. Gefen, 2000, Phys. Rev. B 61, 10247.

Baltin, R., Y. Gefen, G. Hackenbroich, and H.A. Weidenmüller, 1999, Eur. Phys. J. B 10, 119.

Baranger, H. U., 1998, in Nanotechnology, edited by G. Timp (American Institute of Physics, New York), p. 537.

Baranger, H. U., R. A. Jalabert, and A. D. Stone, 1993a, Phys. Rev. Lett. 70, 3876.

Baranger, H.U., R.A. Jalabert and A.D. Stone, 1993b, Chaos 3, 665 .

Baranger, H.U., and P.A. Mello, 1994, Phys. Rev. Lett. 73, 142 .

Baranger, H.U., and P.A. Mello, 1995, Phys. Rev. B 51, 4703.

Baranger, H.U., and P.A. Mello, 1996, Europhys. Lett. 33, 465.

Baranger, H.U., and A.D. Stone, 1989, Phys. Rev. B 40, 8169.

Baranger, H.U., D. Ullmo, and L.I. Glazman, 2000, Phys.

Rev. B 61, R2425.

Beenakker, C.W.J., 1991, Phys. Rev. B 44, 1646.

Beenakker, C.W.J., 1993, Phys. Rev. Lett. 70, 4126.

Beenakker, C.W.J., 1997, Rev. Mod. Phys. 69, 731.

Beenakker, C.W.J., and H. van Houten, 1989, Phys. Rev. Lett. 63, 1857.

Beenakker, C.W.J., and H. van Houten, 1991, Solid State Phys. 44, 1.

Ben-Jacob, E., and Y. Gefen, 1985, Phys. Lett. 108A, 289.

Bergmann, G., 1984, Phys. Rep. 107, 1.

Berkovits, R., 1998, Phys. Rev. Lett. 81, 2128.

Berkovits, R., 1999, Mod. Phys. Lett. B 13, 3841.

Berkovits, R., and B. L. Altshuler, 1997, Phys. Rev. B 55,
5297.

Berkovits, R., and Y. Avishai, 1998, Phys. Rev. Lett. 80, 568. Berkovits, R., and U. Sivan, 1998, Europhys. Lett. 41, 653.

Berman, D., N.B. Zhitenev, R.C. Ashoori, and M. Shayegan, 1999, Phys. Rev. Lett. 82, 161.

Berry, M.J., J.H. Baskey, R.M. Westervelt, and A.C. Gossard, 1994a, Phys. Rev. B 50, 8857.

Berry, M.J., J.H. Katine, R.M. Westervelt, and A.C. Gossard, 1994b, Phys. Rev. B 50, 17721.

Berry, M.V., 1977, J. Phys. A 10, 2083.

Berry, M.V., 1985, Proc. R. Soc. London, Ser. A 400, 229.

Berry, M.V., and M. Robnik, 1986, J. Phys. A 19, 649.

Bertsch, G.F., 1991, J. Phys.: Condens. Matter 3, 373.

Blanter, Ya.M., 1996, Phys. Rev. B 54, 12807.

Blanter, Ya.M., and A.D. Mirlin, 1997, Phys. Rev. E 55, 6514.

Blanter, Ya.M., and A.D. Mirlin, 1998, Phys. Rev. B 57, 4566.

Blanter, Ya.M., A.D. Mirlin, and B.A. Muzykantskii, 1997,

Phys. Rev. Lett. 78, 2449.

Blanter, Ya.M., A.D. Mirlin, and B.A. Muzykantskii, 1998, Phys. Rev. Lett. 80, 4161.

Blatt, J.M., and V.F. Weisskopf, 1952, Theoretical Nuclear Physics (Wiley, New York).

Blümel, R., and U. Smilansky, 1988, Phys. Rev. Lett. 60, 477. Blümel, R., and U. Smilansky, 1989, Physica D 36, 111.

Blümel, R., and U. Smilansky, 1990, Phys. Rev. Lett. 64, 241. Blümel, R., and U. Smilansky, 1992, Phys. Rev. Lett. 69, 217. Bockrath, M., D.H. Cobden, P.L. McEuen, N.G. Chopra, A. Zettl, A. Thess, and R.E. Smalley, 1997, Science 275, 1922.

Bohigas, O., 1991, in Chaos and Quantum Physics, edited by M.-J. Giannoni, A. Voros and J. Zinn-Justin (NorthHolland, Amsterdam).

Bohigas, O., and J. Flores, 1971, Phys. Lett. B 34, 261.

Bohigas, O., and M.-J. Giannoni, 1984, in Mathematical and Computational Methods in Nuclear Physics, edited by J.S. Dehesa, J.M.G. Gomez and A. Polls, Lectures Notes in Physics No. 209 (Springer, Berlin), p. 1.

Bohigas, O., M-J. Giannoni, A.M. Ozorio de Almeida, and C. Schmit, 1995, Nonlinearity 8, 203.

Bohigas, O., M.-J. Giannoni, and C. Schmit, 1984, Phys. Rev. Lett. 52, 1.

Bohigas, O., R.U. Haq, and A. Pandey, 1983, in Nuclear Data for Science and Technology, edited by K.H. Böchhoff (Reidel, Dordrecht), p. 809.

Bohigas, O., R.U. Haq, and A. Pandey, 1985, Phys. Rev. Lett. 54, 1645.

Bohr, A., and B.R. Mottelson, 1969, Nuclear Structure, Vol. I (Benjamin, Reading, MA); 1998 edition by World Scientific, Singapore.

Bonci, L., and R. Berkovits, 1999, Europhys. Lett. 47, 708.

Braun, D., and G. Montambaux, 1995, Phys. Rev. B 52, 13903.

Brézin E., and A. Zee, 1993, Nucl. Phys. B 402, 613.

Brody, T.A., J. Flores, J.B. French, P.A. Mello, A. Pandey, and S.S.M. Wong, 1981, Rev. Mod. Phys. 53, 385.

Brookhaven National Laboratory, 1964, Neutron Cross Sections, BNL 325, Suppl. 2 (Brookhaven National Laboratory, Upton, NY).

Brouwer, P.W., 1995, Phys. Rev. B 51, 16878.

Brouwer, P.W., 1998, Phys. Rev. B 58, R10135.

Brouwer, P.W., and I.L. Aleiner, 1999, Phys. Rev. Lett. 82, 
390.

Brouwer, P.W., and C.W.J. Beenakker, 1994, Phys. Rev. B 50, 11263.

Brouwer, P.W., and C.W.J. Beenakker, 1995, Phys. Rev. B 51, 7739.

Brouwer, P.W., and C.W.J. Beenakker, 1996, J. Math. Phys. 37, 4904.

Brouwer, P.W., and C.W.J. Beenakker, 1997, Phys. Rev. B 55, 4695.

Brouwer, P.W., Y. Oreg, and B.I. Halperin, 1999, Phys. Rev. B 60, R13977.

Bruus, H., C.H. Lewenkopf, and E.R. Mucciolo, 1996, Phys. Rev. B 53, 9968.

Bruus, H., and A.D. Stone, 1994, Phys. Rev. B 50, 18275.

Büttiker, M., 1986a, Phys. Rev. Lett. 57, 1761.

Büttiker, M., 1986b, Phys. Rev. B 33, 3020.

Büttiker, M., 1988a, IBM J. Res. Dev. 32, 63.

Büttiker, M., 1988b, IBM J. Res. Dev. 32, 317.

Chakravarty, S., and A. Schmid, 1986, Phys. Rep. 140, 193.

Chan, I.H., R.M. Clarke, C.M. Marcus, K. Campman, and A.C. Gossard, 1995, Phys. Rev. Lett. 74, 3876.

Chang, A.M., H.U. Baranger, L.N. Pfeiffer, and K.W. West, 1994, Phys. Rev. Lett. 73, 2111.

Chang, A.M., H.U. Baranger, L.N. Pfeiffer, K.W. West, and T.Y. Chang, 1996, Phys. Rev. Lett. 76, 1695.

Choi, K.K., D.C. Tsui, and K. Alavi, 1987, Phys. Rev. B 36, 7551 .

Clarke, R.M., I.H. Chan, C.M. Marcus, C.I. Duruöz, J.S. Harris, Jr., K. Campman, and A.C. Gossard, 1995, Phys. Rev. B 52, 2656.

Cobden, D.H., M. Bockrath, P.L. McEuen, A.G. Rinzler, and R.E. Smalley, 1998, Phys. Rev. Lett. 81, 681.

Cohen, A., K. Richter, and R. Berkovits, 1999, Phys. Rev. B 60, 2536.

Cronenwett, S.M., S.M. Maurer, S.R. Patel, C.M. Marcus, C.I. Duruöz, and J.S. Harris, Jr., 1998, Phys. Rev. Lett. 81, 5904.

Cronenwett, S.M., T.H. Oosterkamp, and L.P. Kouwenhoven, 1998, Science 281, 540.

Cronenwett, S.M., S.R. Patel, C.M. Marcus, K. Campman, and A.C. Gossard, 1997, Phys. Rev. Lett. 79, 2312.

Datta, S., 1995, Electronic Transport in Mesoscopic Systems (Cambridge University, Cambridge).

Davidović, D., and M. Tinkham, 1999, Phys. Rev. Lett. 83, 1644.

Dittrich, T., 1996, Phys. Rep. 271, 268.

Doron, E., U. Smilansky, and A. Frenkel, 1990, Phys. Rev. Lett. 65, 3072.

Doron, E., U. Smilansky, and A. Frenkel, 1991, Physica D 50, 367.

Dupuis, N., and G. Montambaux, 1991, Phys. Rev. B 43, 14390.

Dyugaev, A.M., I.D. Vagner,and P. Wyder, 2000, e-print cond-mat/0005005.

Dyson, F.J., 1962a, J. Math. Phys. 3, 140.

Dyson, F.J., 1962b, J. Math. Phys. 3, 157, 166.

Dyson, F.J., 1962c, J. Math. Phys. 3, 1191.

Dyson, F.J., 1962d, J. Math. Phys. 3, 1199.

Dyson, F.J., 1970, Commun. Math. Phys. 19, 235.

Dyson, F.J.,and M.L. Mehta, 1963, J. Math. Phys. 4, 701.
Echternach, P.M., M.E. Gersheson, and H.M. Bozler, 1993, Phys. Rev. B 48, 11516.

Economou, E.N., and C.M. Soukoulis, 1981, Phys. Rev. Lett. 46, 618 .

Edwards, J.T., and D.J. Thouless, 1972, J. Phys. C 5, 807.

Efetov, K.B., 1983, Adv. Phys. 32, 53.

Efetov, K.B., 1995, Phys. Rev. Lett. 74, 2299.

Efetov, K.B., 1997, Supersymmetry in Disorder and Chaos (Cambridge University, Cambridge).

Ericson, T., 1960, Phys. Rev. Lett. 5, 430.

Ericson, T., 1963, Ann. Phys. (N.Y.) 23, 390.

Ericson, T., and T. Mayer-Kuckuk, 1966, Annu. Rev. Nucl. Sci. 16, 183.

Fal'ko, V.I., and K.B. Efetov, 1994, Phys. Rev. B 50, 11267. Fal'ko, V.I., and K.B. Efetov, 1996, Phys. Rev. Lett. 77, 912. Ferry, D.K., and S.M. Goodnick, 1997, Transport in Nanostructures (Cambridge University, Cambridge).

Feshbach, H., 1992, Theoretical Nuclear Physics: Nuclear Reactions (Wiley, New York).

Fetter, A.L., and J.D. Walecka, 1971, Quantum Theory of Many-Particle Systems (McGraw-Hill, New York).

Fisher, D.S., and P.A. Lee, 1981, Phys. Rev. B 23, 6851.

Flambaum, V.V., A.A. Gribakina, G.F. Gribakin and M.G. Kozlov, 1994, Phys. Rev. A 50, 267.

Flambaum, V.V., G.F. Gribakin, and F.M. Izrailev, 1996, Phys. Rev. E 53, 5729.

Flensberg, K., 1993, Phys. Rev. B 48, 11156; 1994, Physica B 203, 432.

Folk, J.A., S.R. Patel, S.F. Godijn, A.G. Huibers, S.M. Cronenwett, C.M. Marcus, K. Campman, and A.C. Gossard, 1996, Phys. Rev. Lett. 76, 1699.

Folk, J.A., S.R. Patel, C.M. Marcus, C.I. Duruöz, and J.S. Harris, Jr., 2000, "Decoherence in nearly-isolated quantum dots," e-print cond-mat/0008052.

Frahm, K.M., 1995, Europhys. Lett. 30, 457.

French, J.B., and V.K.B. Kota, 1982, Ann. Rev. Nucl. Part. Sci. 32, 35.

French, J.B., V.K.B. Kota, A Pandey and S. Tomsovic, 1985, Phys. Rev. Lett. 54, 2313.

French, J.B., V.K.B. Kota, A Pandey and S. Tomsovic, 1988, Ann. Phys. (N.Y.) 181, 198.

French, J.B., and S.S.M. Wong, 1970, Phys. Lett. B 33, 449. Friedman, W.A., and P.A. Mello, 1985, Ann. Phys. (N.Y.) 161, 276.

Fulton, T.A., and G.J. Dolan, 1987, Phys. Rev. Lett. 59, 109. Furusaki, A., and K.A. Matveev, 1995a, Phys. Rev. Lett. 75, 709 .

Furusaki, A., and K.A. Matveev, 1995b, Phys. Rev. B 52, 16676.

Fyodorov, Y.V., and H.-J. Sommers, 1996a, Phys. Rev. Lett. 76, 4709.

Fyodorov, Y.V., and H.-J. Sommers, 1996b, JETP Lett. 63, 1026.

Fyodorov, Y.V., and H.-J. Sommers, 1997, J. Math. Phys. 38, 1918.

Garg, J.B., J. Rainwater, J.S. Petersen and W.W. Havens, Jr., 1964, Phys. Rev. 134, B985.

Gaudin, M., 1961, Nucl. Phys. 25, 447.

Gershenson, M.E., 1999, Ann. Phys. 8, 559.

Giaever, I., and H.R. Zeller, 1968, Phys. Rev. Lett. 20, 1504. 
Giannoni, M.-J., A. Voros, and J. Zinn-Justin, 1991, eds., Chaos and Quantum Physics (North-Holland, Amsterdam). Glazman, L.I., and K.A. Matveev, 1988, JETP Lett. 48, 445. Glazman, L.I., and K.A. Matveev, 1990a, JETP Lett. 51, 484.

Glazman, L.I., and K.A. Matveev, 1990b, Sov. Phys. JETP $\mathbf{7 1}, 1031$.

Glazman, L.I., and M.E. Raikh , 1988, JETP Lett. 47, 452.

Glazman, L.I., and R.I. Shekther, 1989, J. Phys.: Condens. Matter 1, 5811.

Goldberg, J., U. Smilansky, M.V. Berry, W. Schweizer, G. Wunner, and G. Zeller, 1991, Nonlinearity 4, 1.

Goldhaber-Gordon, D., H. Shtrikman, D. Mahalu, D. AbuschMagder, U. Meirav, and M.A. Kastner, 1998, Nature (London) 391, 156.

Gopar, V.A., P.A. Mello, and M. Büttiker, 1996, Phys. Rev. Lett. 77, 3005.

Gor'kov, L.P., and G.M. Eliashberg, 1965, Sov. Phys. JETP 21, 940.

Gor'kov, L.P., A.I. Larkin, and D.E. Khmelnitskii, 1979, JETP Lett. 30, 228.

Gossiaux, P.B., Z. Pluhar̆, and H. A. Weidenmüller, 1998, Ann. Phys. (N.Y.) 268, 273.

Greenwood, D.A., 1958, Proc. Phys. Soc. London 71, 585.

Guhr, T., A. Müller-Groeling, and H.A. Weidenmüller, 1998, Phys. Rep. 299, 190.

Gutzwiller, M.C., 1967, J. Math. Phys. 8, 1979.

Gutzwiller, M.C., 1969, J. Math. Phys. 10, 1004.

Gutzwiller, M.C., 1970, J. Math. Phys. 11, 1791.

Gutzwiller, M.C., 1971, J. Math. Phys. 12, 343.

Gutzwiller, M.C., 1990, Chaos in Classical and Quantum Mechanics (Springer, New York).

Hackenbroich, G., W.D. Heiss and H.A. Weidenmüller, 1997, Phys. Rev. Lett. 79, 127.

Hackenbroich, G., and H.A. Weidenmüller, 1995, Phys. Rev. Lett. 74, 4118.

Hannay, J.H., and A.M. Ozorio de Almeida, 1984, J. Phys. A $\mathbf{1 7}, 3429$.

Haq, R.U., A. Pandey, and O. Bohigas, 1982, Phys. Rev. Lett. 48, 1086.

Hauser, W., and H. Feshbach, 1952, Phys. Rev. 87, 366.

Hortikar, S., and M. Srednicki, 1998, Phys. Rev. Lett. 80, 1646.

Hua, L.K., 1963, Harmonic Analysis of Functions of Several Complex Variables in the Classical Domains (American Mathematical Society, Providence, RI).

Hubbard, J., 1963, Proc. R. Soc. London, Ser. A 276, 238.

Huibers, A.G., M. Switkes, C.M. Marcus, P.W. Brouwer, C.I. Duruöz, and J.S. Harris, Jr., 1998, Phys. Rev. Lett. 81, 1917.

Huibers, A.G., M. Switkes, C.M. Marcus, K. Campman, and A.C. Gossard, 1998, Phys. Rev. Lett. 81, 200.

Iida, S., H.A. Weidenmüller, and J.A. Zuk, 1990a, Phys. Rev. Lett. 64, 583 .

Iida, S., H.A. Weidenmüller, and J. A. Zuk, 1990b, Ann. Phys. (N.Y.) 200, 219.

Imry, Y., 1986a, Europhys. Lett. 1, 249.

Imry, Y., 1986b, in Directions in Condensed Matter Physics, vol. I, edited by G. Grinstein and G. Mazenko (World Scientific, Singapore).
Imry, Y., 1996, Introduction to Mesoscopic Physics (Oxford University, Oxford).

Imry, Y., and R. Landauer, 1999, Rev. Mod. Phys. 71, S306. Jacquod, Ph., and D.L. Shepelyansky, 1997, Phys. Rev. Lett. 79, 1837.

Jacquod, Ph., and A.D. Stone, 2000, Phys. Rev. Lett. 84, 3938.

Jalabert, R.A., H.U. Baranger, and A.D. Stone, 1990, Phys. Rev. Lett. 65, 2442.

Jalabert, R. A., J.-L. Pichard, and C. W. J. Beenakker, 1994, Europhys. Lett. 27, 255.

Jalabert, R.A., A.D. Stone, and Y. Alhassid, 1992, Phys. Rev. Lett. 68, 3468.

Jensen, R.V., 1991, Chaos 1, 101.

Johnson, A.T., L.P. Kouwenhoven, W. de Jong, N.C. van der Vaart, C.J.P.M. Harmans, and C.T. Foxon, 1992, Phys. Rev. Lett. 69, 1592.

Johnson, C.W., G.F. Bertsch, and D.J. Dean, 1998, Phys. Rev. Lett. 80, 2749.

Kamenev, A., and Y. Gefen, 1997, Chaos, Solitons, and Fractals 8, 1229.

Kaminski, A., I.L. Aleiner, and L.I. Glazman, 1998, Phys. Rev.Lett. 81, 685.

Kaminski, A., and L.I. Glazman, 1999, Phys. Rev. B 59, 9798.

Kaminski, A., and L.I. Glazman, 2000, Phys. Rev. B 61, 15927.

Kanzepier, E., and V. Freilikher, 1996, Phys. Rev. B 54, 8737.

Kaplan, L., and E.J. Heller, 1998, Phys. Rev. Lett. 80, 2582.

Kastner, M.A., 1992, Rev. Mod. Phys. 64, 849.

Kastner, M.A., 1993, Phys. Today 46 (1), 24.

Keller, M.W., O. Millo, A. Mittal, D.E. Prober, and R.N. Sacks, 1994, Surf. Sci. 305, 501.

Keller, M.W., A. Mittal, J.W. Sleight, R.G. Wheeler, D.E. Prober, R.N. Sacks, and H. Shtrikmann, 1996, Phys. Rev. B 53, 1693.

Ketzmerick, R., 1996, Phys. Rev. B 54, 10841.

Khmelnitskii, D.E., 1984, Physica B 126, 235.

Kittel, C., 1987, Quantum Theory of Solids (Wiley, New York).

Kogan, E., and M. Kaveh, 1995, Phys. Rev. B 51, 16400.

Kondo, J., 1964, Prog. Theor. Phys. 32, 37.

Koopmans, T., 1934, Physica (Amsterdam) 1, 104.

Koulakov, A.A., F.G. Pikus, and B.I. Shklovskii, 1997, Phys. Rev. B 55, 9223.

Koulakov, A.A., and B.I. Shklovskii, 1998, Phys. Rev. B 57, 2352.

Kouwenhoven, L.P., C.M. Marcus, P.L. Mceuen, S. Tarucha, R.M. Wetervelt, and N.S. Wingreen, 1997, in Mesoscopic electron transport, NATO Advanced Study Institute, Series E, No. 345, edited by L. L. Sohn, L. P. Kouwenhoven, and G. Schoen (Kluwer, Dordrecht).

Kouwenhoven, L.P., and P.L. McEuen, 1998, in Nanotechnology, edited by G. Timp (AIP, New York), p. 471.

Kouwenhoven, L.P., T.H. Oosterkamp, M.W.S. Danoesastro, M. Eto, D.G. Austing, T. Honda, and S. Tarucha, 1997, Science 278, 1788.

Krieger, T.J., and C.E. Porter, 1963, J. Math. Phys. 4, 1272. Kubo, R., 1957, J. Phys. Soc. Jpn. 12, 570.

Kulik, I.O., and R.I. Shekhter, 1975, Sov. Phys. JETP 41, 308. 
Kurdak, C., A.M. Chang, A. Chin, and T.Y. Chang, 1992, Phys. Rev. B 46, 6846.

Kurland, I.L., I.L. Aleiner, and B.L. Altshuler, 2000, "Mesoscopic Stoner instability," e-print cond-mat/0004205.

Kurland, I.L., R. Berkovits, and B.L. Altshuler, 2000, "Conductance peak motion due to a magnetic field in weakly coupled chaotic quantum dots," e-print cond-mat/0005424.

Landauer, R., 1957, IBM J. Res. Dev. 1, 223.

Landauer, R., 1970, Philos. Mag. 21, 863.

Lane, A.M., and R.G. Thomas, 1958, Rev. Mod. Phys. 30, 257.

Langer, J.S., and T. Neal, 1966, Phys. Rev. Lett. 16, 1984.

Lee, P.A., and T.V. Ramakrishnan, 1985, Rev. Mod. Phys. 57, 287.

Lee, P.A., and A.D. Stone, 1985, Phys. Rev. Lett. 55, 1622.

Lee, P.A., A.D. Stone, and H. Fukuyama, 1987, Phys. Rev. B 35, 1039.

Levit, S., and D. Orgad, 1999, Phys. Rev. B 60, 5549.

Leyronas, X., J. Tworzydlo, and C.W.J. Beenakker, 1999, Phys. Rev. Lett. 82, 4894.

Likharev, K.K., and A.B. Zorin, 1985, J. Low Temp. Phys. $\mathbf{5 9}, 347$.

Lin, J.J., and N. Giordano, 1986, Phys. Rev. B 33, 1159.

Lüscher, S., T. Heinzel, K. Ensslin, W. Wegscheider, M. Bichler, 2000, "Signatures of spin pairing in a quantum dot in the Coulomb-blockade regime," cond-mat/0002226.

Mahaux, C., and H.A. Weidenmüller, 1969, Shell-Model Approach to Nuclear Reactions (North-Holland, Amsterdam).

Marcus, C.M., S. R. Patel, A. G. Huibers, S.M. Cronenwett, M. Switkes, I.H. Chan, R.M. Clarke, J.A. Folk, S.F. Godijn, K. Campman, and A.C. Gossard, 1997, Chaos, Solitons, and Fractals 8, 1261.

Marcus, C.M., A.J. Rimberg, R.M. Westervelt, P.F. Hopkins, and A.C. Gossard, 1992, Phys. Rev. Lett. 69, 506.

Marcus, C.M., R.M. Westervelt, P.F. Hopkins, and A.C. Gossard, 1993a, Phys. Rev B 48, 2460.

Marcus, C.M., R.M. Westervelt, P.F. Hopkins, and A. C. Gossard, 1993b, Chaos 3, 643.

Marcus, C.M., R.M. Westervelt, P.F. Hopkins and A.C. Gossard, 1994, Surf. Sci. 305, 480.

Matveev, K.A., 1991, Sov. Phys. JETP 72, 892.

Matveev, K.A., 1995, Phys. Rev. B 51, 1743.

Maurer, S.M., S.R. Patel, C.M. Marcus, C.I. Duruöz, and J.S. Harris, Jr., 1999, Phys. Rev. Lett. 83, 1403.

McEuen, P.L., 1998, Nature (London) 393, 6680.

McEuen, P.L., N.S. Wingreen, E.B. Foxman, J. Kinaret, U. Meirav, M.A. Kastner, and Y. Meir, 1993, Physica B 189, 70.

Mehta, M.L., 1960, Nucl. Phys. 18, 395.

Mehta, M.L., 1971, Commun. Math. Phys. 20, 245.

Mehta, M.L., 1991, Random Matrices, second edition (Academic, New York).

M.L. Mehta, and M. Gaudin, 1960, Nucl. Phys. 18, 420.

Mehta, M.L., and A. Pandey, 1983, J. Phys. A 16 2655; L601.

Meir, Y., and N. S. Wingreen, 1992, Phys. Rev. Lett. 68, 2512.

Meir, Y., N.S. Wingreen, and P.A. Lee, 1991, Phys. Rev. Lett. 66, 3048.

Meir, Y., N.S. Wingreen, and P.A. Lee, 1993, Phys. Rev. Lett. 70, 2601.
Meirav, U., and E.B. Foxman, 1995, Semicond. Sci. Technol. 10, 255 .

Meirav, U., M.A. Kastner and S.J. Wind, 1990, Phys. Rev. Lett. 65, 771.

Mejia-Monasterio, C., J. Richert, T. Rupp, and H.A. Weidenmüller, 1998, Phys. Rev. Lett. 81, 5189.

Mello, P.A., 1995, in Mesoscopic Quantum Physics, edited by E. Akkermans, G. Montambaux, J.-L. Pichard, and J. Zinn-Justin (North-Holland, Amsterdam), p. 435.

Mello, P.A., and H.U. Baranger, 1995, Physica A 220, 15.

Mello, P.A., and H.U. Baranger, 1999, Waves Random Media 9, 105.

Mello, P.A., P. Pereyra, and T.H. Seligman, 1985, Ann. Phys. (N.Y.) 161, 254.

Mirlin, A.D., 1997, in Supersymmetry and Trace Formulae, Proceedings of the NATO Advanced Study Institute, Series B, No. 370, edited by I.V. Lerner, V.P. Keating, and D.E. Khmelnitskii (Kluwer Academic, New York).

Mirlin, A.D., 2000, Phys. Rep. 326, 260.

Mitchell, D., Y. Alhassid, and D. Kusnezov, 1996, Phys. Lett. A 215, 21.

Mohanty, P., E.M.Q. Jariwala, and R.A. Webb, 1997, Phys. Rev. Lett. 78, 3366.

Montambaux, G., 1997, in Quantum Fluctuations, Proceedings of the Les Houches Summer School, Session LXIII, edited by S. Reynaud, E. Giacobino, and J. Zinn-Justin (North-Holland, Amsterdam), p. 387.

Montambaux, G., D. Poilblanc, J. Bellissard, and C. Sire, 1993, Phys. Rev. Lett. 70, 497.

Mucciolo, E.R., V.N. Prigodin, and B.L. Altshuler, 1995, Phys. Rev. B 51, 1714.

Muzykantskii, B.A., and D.E. Khmelnitskii, 1995, JETP Lett. 62, 76 .

Narayan, O., and B.S. Shastry, 1993, Phys. Rev. Lett. 71, 2106.

Narimanov, E.E., N.R. Cerruti, H.U. Baranger, and S. Tomsovic, 1999, Phys. Rev. Lett. 83, 2640.

Ng, T.K., and P.A. Lee, 1988, Phys. Rev. Lett. 61, 1768.

Oosterkamp, T.H., L.P. Kouwenhoven, A.E.A. Koolen, N.C. van der Vaart, and C.J.P.M. Harmans, 1997, Phys. Rev. Lett. 78, 1536.

Oreg, Y, and Y. Gefen, 1997, Phys. Rev. B 55, 13726.

Ormand, W.E., D.J. Dean, C.W. Johnson, G.H. Lang, and S.E. Koonin, 1994, Phys. Rev. C 49, 1422.

Pandey, A., 1979, Ann. Phys. (N.Y.) 119, 170.

Pandey, A., and M.L. Mehta, 1983, Commun. Math. Phys. 87, 49 .

Patel, S.R., S.M. Cronenwett, D.R. Stewart, C. M. Marcus, C. I. Duruöz, J. S. Harris, Jr., K. Campman, and A.C. Gossard, 1998, Phys. Rev. Lett. 80, 4522.

Patel, S.R., D.R. Stewart, C. M. Marcus, M. Gökçedă̆, Y. Alhassid, A. D. Stone, C. I. Duruöz, and J. S. Harris, Jr., 1998, Phys. Rev. Lett. 81, 5900.

Pereyra, P., and P.A. Mello, 1983, J. Phys. A 16, 237.

Pichaureau, P., and R.A. Jalabert, 1999, Eur. Phys. J. B 9, 299.

Pines, D., and P. Nozières, 1966, The Theory of Quantum Liquids, Vol. 1 (Addison-Wesley, New York).

Pluhar, Z., and H.A. Weidenmüller, 1999, Ann. Phys. (N.Y.) 272, 295. 
Pluhar̆, Z., H.A. Weidenmüller, J. A. Zuk, and C. H. Lewenkopf, 1994, Phys. Rev. Lett. 73, 2115.

Pluhar̆, Z., H.A. Weidenmüller, J.A. Zuk, C.H. Lewenkopf, and F.J. Wegner, 1995, Ann. Phys. (N.Y.) 243, 1.

Porath, D., and O. Millo, 1996, J. Appl. Phys. 81, 2241.

Porter, C.E., 1965, Statistical Theory of Spectra: Fluctuations (Academic, New York).

Porter, C.E., and N. Rosenzweig, 1960, Phys. Rev. 120, 1698.

Prigodin, V.N., 1995, Phys. Rev. Lett. 74, 1566.

Prigodin, V.N., K.B. Efetov, and S. Iida, 1993, Phys. Rev. Lett. 71, 1230.

Ralph, D.C., C.T. Black, and M. Tinkham, 1997, Phys. Rev. Lett. 78, 4087.

Reed, M.A., J.N. Randall, R.J. Aggarwall, R.J. Matyi, T.M. Moore, and A.E. Wetsel, 1988, Phys. Rev. Lett. 60, 535.

Reichel, L.E., 1992, The Transition to Chaos in Conservative Classical Systems: Quantum Manifestations (Springer, New York).

Robnik, M.,1983, J. Phys. A 17, 1049.

Sachrajda, A.S., R. Ketzmerick, C. Gould, Y. Feng, P.J. Kelly, A. Delage and Z. Wasilewski, 1998, Phys. Rev. Lett. 80, 1948.

Schuster, R., E. Buks, M. Heiblum, D. Mahalu, V. Umansky, and H. Shtrikman, 1997, Nature (London) 385, 417.

Scott-Thomas, J.H.F., S.B. Field, M.A. Kastner, H.I. Smith, and D.A. Antonadis, 1989, Phys. Rev. Lett. 62, 583.

Shannon, C.E., 1948, Bell Syst. Tech. J. 27, 397; 623.

Shekhter, R.I., 1972, Sov. Phys. JETP 36, 747.

Shutenko, T.A., I.L. Aleiner, and B.L. Altshuler, 2000, Phys. Rev. B 61, 10366.

Simmel, F., D. Abusch-Magder, D.A. Wharam, M.A. Kastner,and J.P. Kotthaus, 1999, Phys. Rev. B 59, R10441.

Simmel, F., T. Heinzel, and D.A. Wharam, 1997, Europhys. Lett. 38, 123.

Simons, B.D., and B.L. Altshuler, 1993a, Phys. Rev. Lett. 70, 4063.

Simons, B.D., and B.L. Altshuler, 1993b, Phys. Rev. B 48, 5422.

Sivan, U., R. Berkovits, Y. Aloni, O. Prus, A. Auerbach, and G. Ben-Yoseph, 1996, Phys. Rev. Lett. 77, 1123.

Sivan, U., F.P. Milliken, K. Milkove, S. Rishton, Y. Lee, J.M. Hong, V. Boegli, D. Kern, and M. DeFranza, 1994, Europhys. Lett. 25, 605.

Smilansky, U., 1990, in Chaos and Quantum Physics, edited by M.-J. Giannoni, A. Voros, and J. Zinn-Justin (NorthHolland, Amsterdam), p. 371.

Sommers, H.-J., and S. Iida, 1994, Phys. Rev. E 49, R 2513. Srednicki, M., 1996, Phys. Rev. E 54, 954.

Stewart, D.R., D. Sprinzak, C.M. Marcus, C.I. Duruöz, and J.S. Harris, Jr., 1997, Science 278, 1784.

Stone, A.D., 1985, Phys. Rev. Lett. 54, 2692.

Stone, A.D., 1995, in Mesoscopic Quantum Physics, edited by E. Akkermans, G. Montambaux, J.-L. Pichard, and J. Zinn-Justin (North-Holland, Amsterdam).

Stone, A.D., and H. Bruus, 1993, Physica B 189, 43.

Stone, A.D., and H. Bruus, 1994, Surf. Sci. 305, 490.

Stone, A.D., P.A. Mello, K.A. Muttalib, and J.-L. Pichard, 1991, in Mesoscopic Phenomena in Solids, edited by B.L. Altshuler, P.A. Lee, and R.A. Webb (North-Holland, Amsterdam), p. 369.
Stone, A.D., and A. Szafer, 1988, IBM J. Res. Dev. 32, 384.

Stopa, M., 1996, Phys. Rev. B 54, 13767.

Stopa, M., 1998, Physica B 251, 228.

Switkes, M., C.M. Marcus, K. Campman, and A.C. Gossard, 1999, Science 283, 1905.

Szafer, A., and B.L. Altshuler, 1993, Phys. Rev. Lett. 70, 587.

Taniguchi, N., A.V. Andreev, and B.L. Altshuler, 1995, Europhys. Lett. 29, 515 .

Tans, S.J., M.H. Devoret, H. Dai, A. Thess, R.E. Smalley, L.J. Geerligs, and C. Dekker, 1997, Nature (London) 386, 474.

Tarucha, S., D.G. Austing, T. Honda, R.J. van der hage, and L.P. Kouwenhoven, 1996, Phys. Rev. Lett. 77, 3613.

Thomas, R.G., and C.E. Porter, 1956, Phys. Rev. 104, 483.

Thouless, D.J., 1974, Phys. Rep. 13, 93.

Thouless, D.J., 1977, Phys. Rev. Lett. 39, 1167.

Ullah, N., 1963, J. Math. Phys. 4, 1279.

Ullah, N., 1967, J. Math. Phys. 8, 1095.

Vallejos, R.O., C.H. Lewenkopf and E.R. Mucciolo, 1998, Phys. Rev. Lett. 81, 677.

van Houten, H, C.W.J. Beenakker, and A.A.M. Staring, 1992, in Single-Charge Tunneling, edited by H. Grabert and M.H. Devoret (Plenum, New York), p. 167.

van Kampen, N.G., 1981, Stochastic Processes in Physics and Chemistry (North-Holland, Amsterdam).

van Langen, S.A., P.W. Brouwer, and C.W.J. Beenakker, 1997, Phys. Rev. E 55, R1.

Verbaarschot, J.J.M., H.A. Weidenmüller, and M.R. Zirnbauer, 1985, Phys. Rep. 129, 367.

von Brentano, P., J. Ernst, O. Hausser, T. Mayer-Kuckuk, A. Richter, and W. von Witsch, 1964, Phys. Lett. 9, 48.

Walker, P.N., Y. Gefen, and G. Montambaux, 1999, Phys. Rev. Lett. 82, 5329.

Walker, P.N., G. Montambaux, and Y. Gefen, 1999, Phys. Rev. B 60, 2541.

Washburn, S., and R.A. Webb, 1993, Rep. Prog. Phys. 55, 1311.

Weisskopf, V., 1937, Phys. Rev. 52, 295.

Westervelt, R.M., 1998, in Nanotechnology, edited by G. Timp (AIP, New York), p. 589.

Wigner, E.P., 1951, Ann. Math. 53, 36.

Wigner, E.P., 1955, Ann. Math. 62, 548.

Wigner, E.P., 1957, Ann. Math. 65, 203.

Wigner, E.P., 1958, Ann. Math. 67, 325.

Wigner, E.P., 1959, Group Theory and its Applications to the Quantum Mechanics of Atomic Spectra (Academic, New York).

Wigner, E.P., and L. Eisenbud, 1947, Phys. Rev. 72, 29.

Wilkinson, M., 1989, J. Phys. A 22, 2795.

Wilkinson, M., and P.N. Walker, 1995, J. Phys. A 28, 6143.

Yacoby, A., M. Heiblum, D. Mahalu, and H. Shtrikman, 1995, Phys. Rev. Lett. 74, 4047.

Zelevinsky, V., B.A. Brown, N. Frazier, and M. Horoi, 1996, Phys. Rep. 276, 85.

Zhitenev, N.B., R.C. Ashoori, L.N. Pfeiffer, and K.W. West, 1997, Phys. Rev. Lett. 79, 2308.

Zhou, F., B. Spivak, and B.L. Altshuler, 1999, Phys. Rev. Lett. 82, 608.

Zyczkowski, K., and G. Lenz, 1991, Z. Phys. B 82, 299. 